\title{
Sicherung von bevorrechtigten umlaufenden Radwegen an innerörtlichen Kreisverkehren
}

Lothar Bondzio

Margarethe Scheit

Betina Berghaus

Nicolai Bissantz

Jean Emmanuel Bakaba

Jörg Ortlepp 


\title{
Sicherung von bevorrechtigten umlaufenden Radwegen an innerörtlichen Kreisverkehren
}

\author{
Dr.-Ing. Lothar Bondzio \\ Dipl.-Ing. Margarethe Scheit \\ Dr. Betina Berghaus \\ Dr. Nicolai Bissantz \\ Dr.-Ing. Jean Emmanuel Bakaba \\ Dipl.-Ing. Jörg Ortlepp
}

Unfallforschung der Versicherer 


\section{Impressum}

Gesamtverband der Deutschen Versicherungswirtschaft e. V. Unfallforschung der Versicherer

Wilhelmstraße 43/43G, 10117 Berlin

Postfach 0802 64, 10002 Berlin

E-Mail: unfallforschung@gdv.de

Internet: www.udv.de

Facebook: www.facebook.com/unfallforschung

Twitter: @unfallforschung

YouTube: www.youtube.com/unfallforschung

ISBN-Nr.: 978-3-939163-76-3

Redaktion: Dr.-Ing. Jean Emmanuel Bakaba

Erschienen: 04/2017 


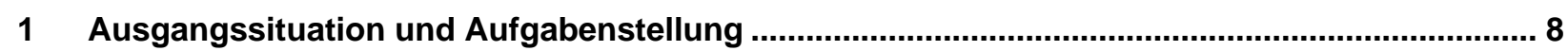

2 Literaturübersicht zur Führung von Radwegen an Kreisverkehren .......................................... 9

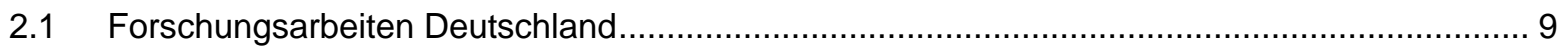

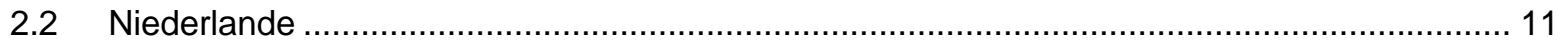

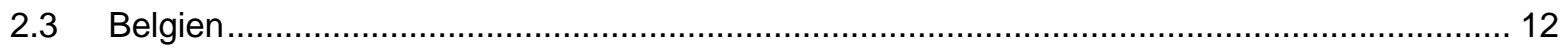

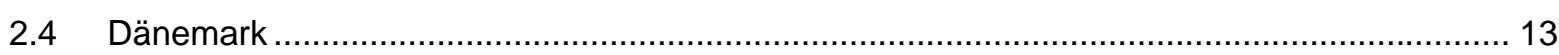

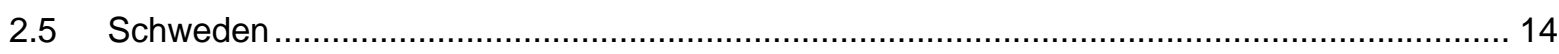

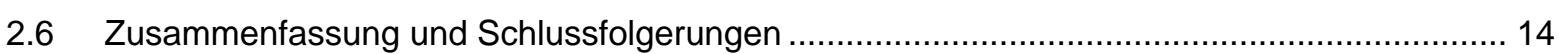

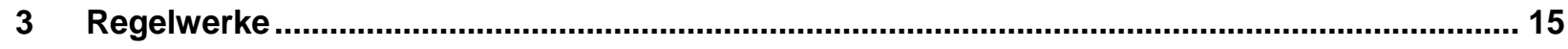

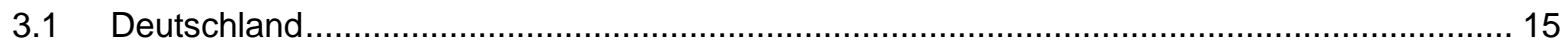

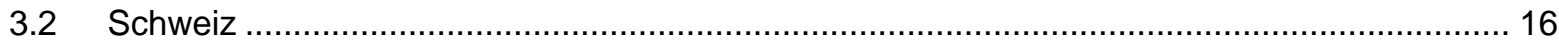

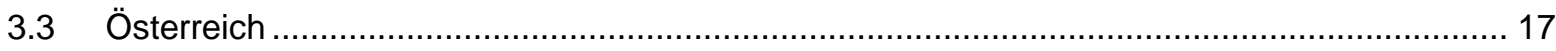

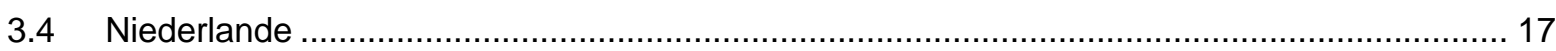

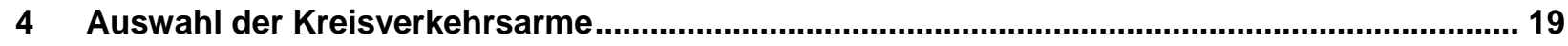

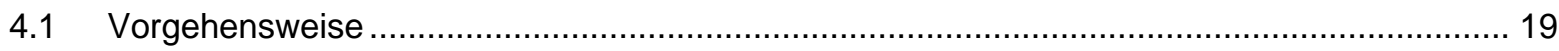

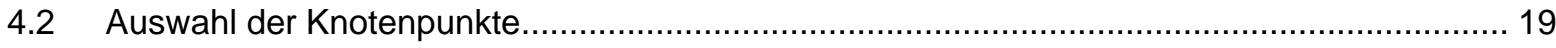

4.3 Merkmale der ausgewählten Knotenpunkte .................................................................. 20

4.3.1 Radwegeführung über die Kreisverkehrsarme ..................................................... 20

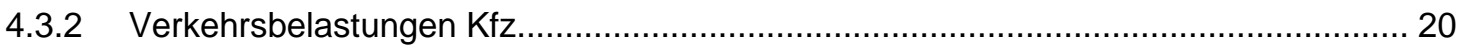

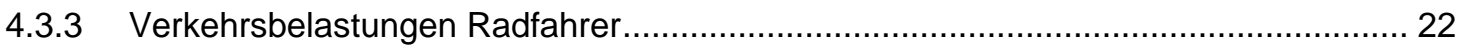

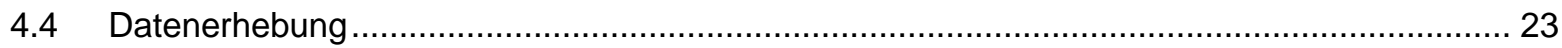

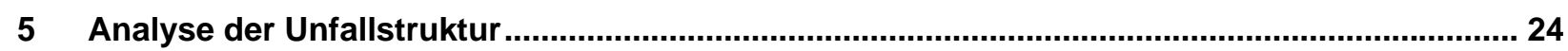

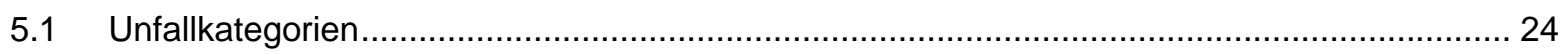

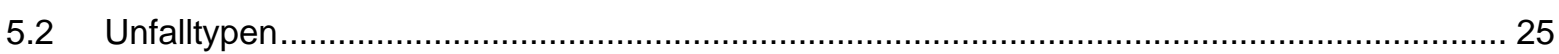

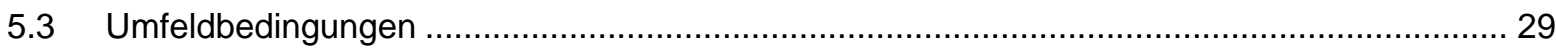

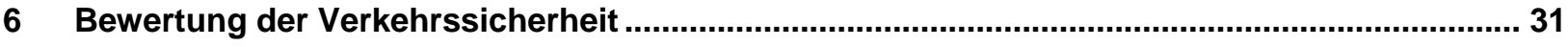

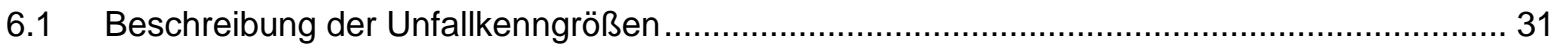

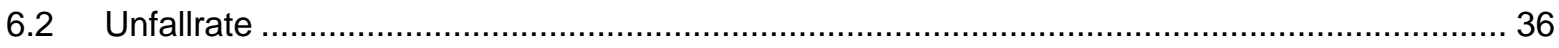

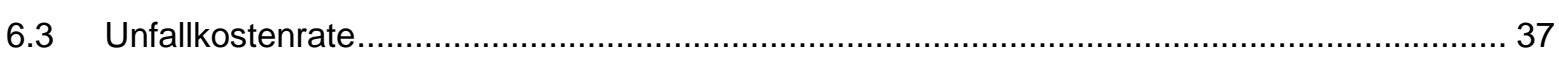

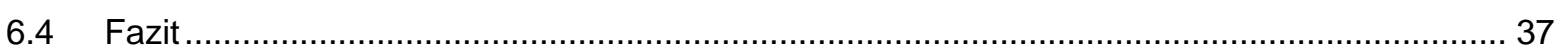




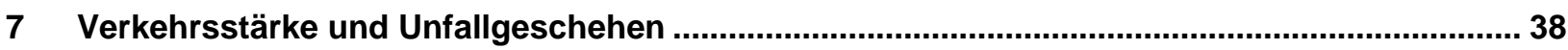

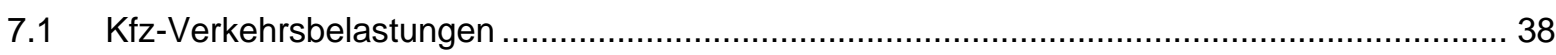

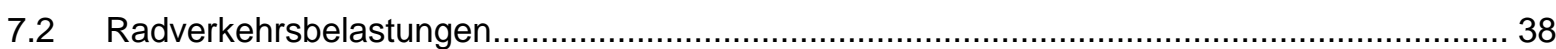

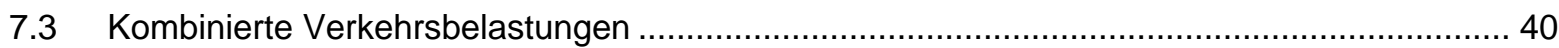

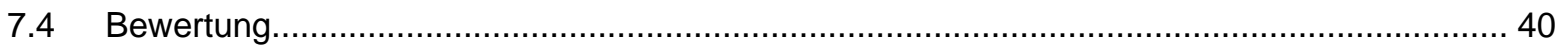

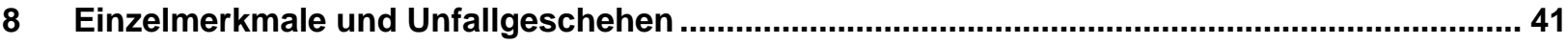

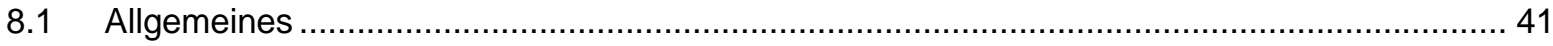

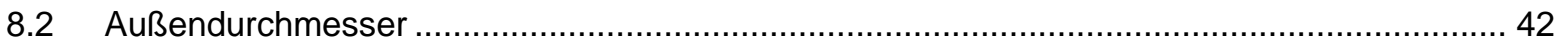

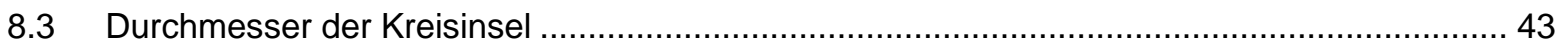

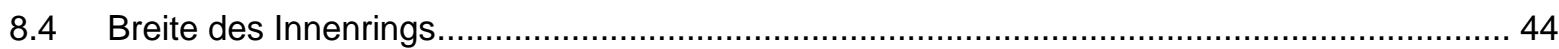

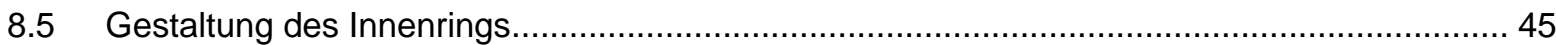

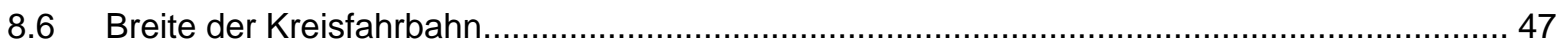

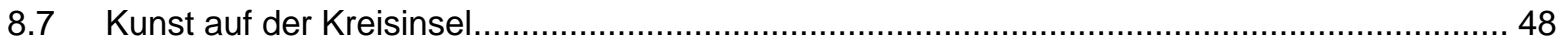

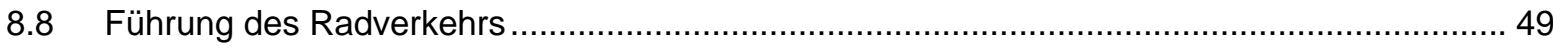

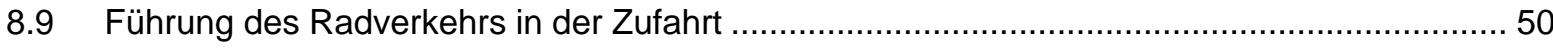

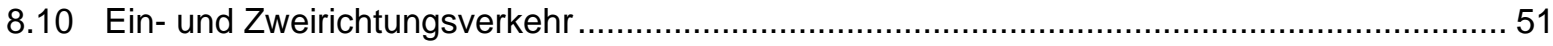

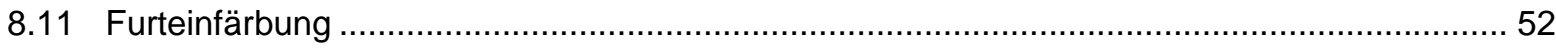

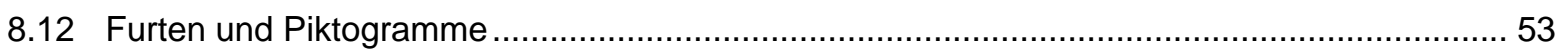

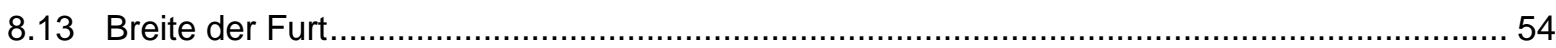

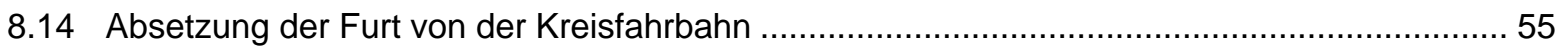

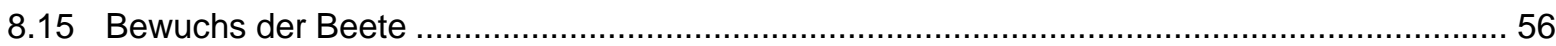

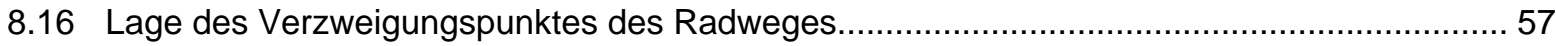

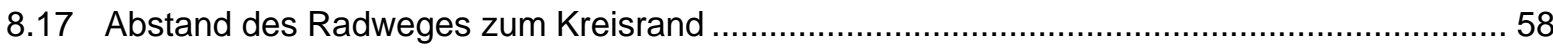

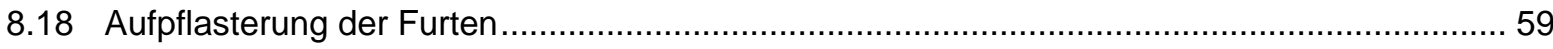

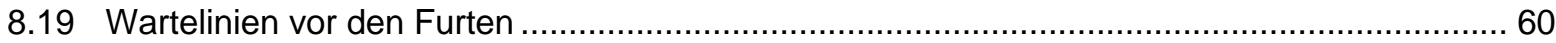

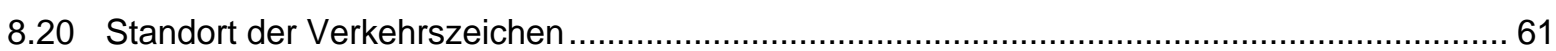

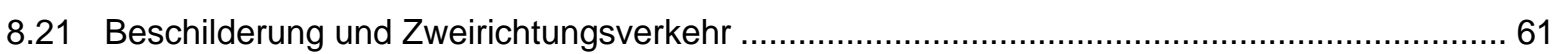

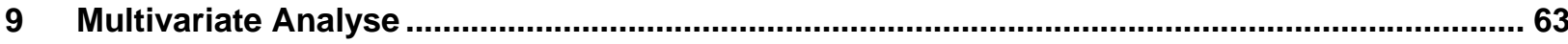

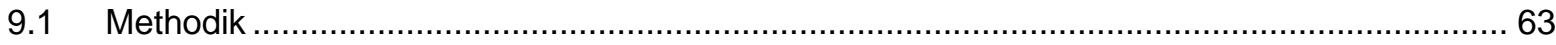

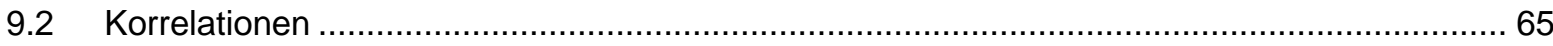

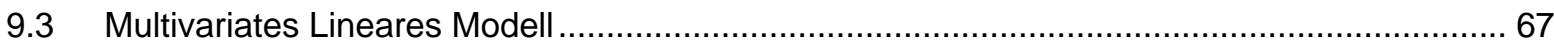

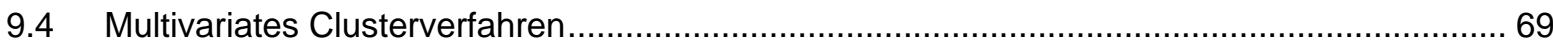

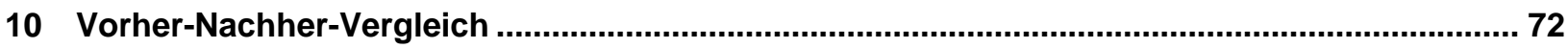

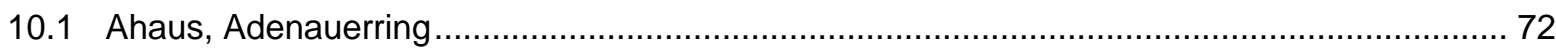

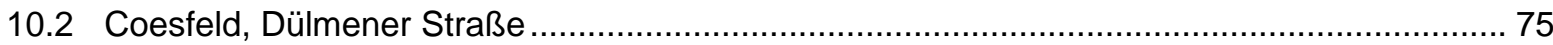




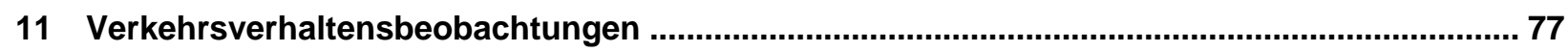

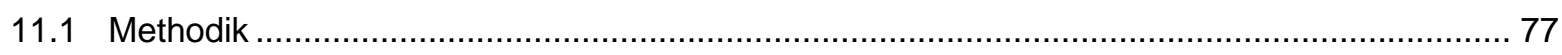

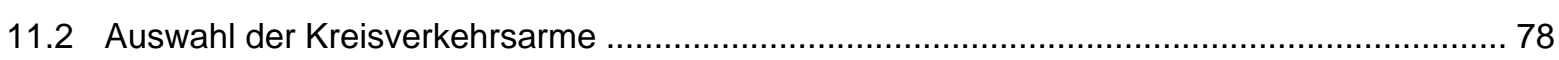

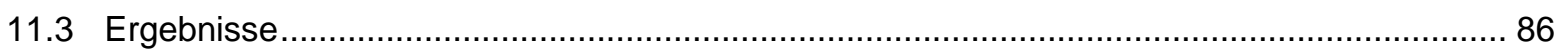

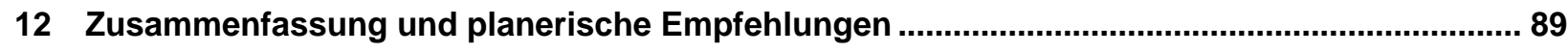

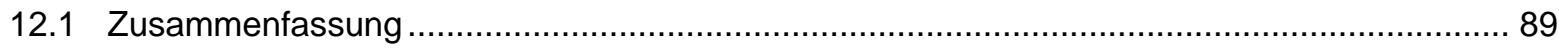

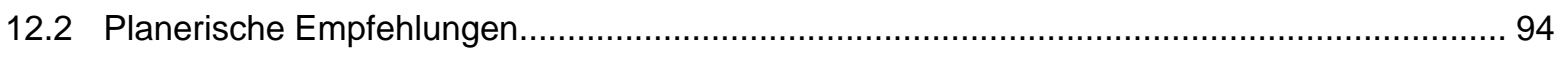

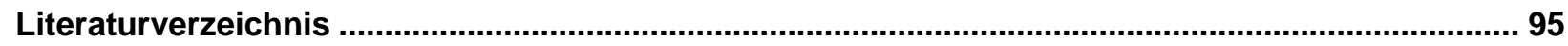




\section{Ausgangssituation und Aufgabenstellung}

Kleine Kreisverkehre haben sich in zahlreichen in- und ausländischen Untersuchungen als ausgesprochen sichere Knotenpunktformen erwiesen. Durch einen Umbau von vorher sicherheitsproblematischen Knotenpunkten in Kreisverkehre lässt sich im Mittel eine deutliche Erhöhung der Verkehrssicherheit erreichen.

Auch für einstreifige Kreisverkehre innerhalb bebauter Gebiete weisen die vorliegenden in- und ausländischen Untersuchungen ein insgesamt hohes Verkehrssicherheitsniveau aus. Zahlreiche Untersuchungen zeigen jedoch auch, dass die Radfahrer am wenigsten vom Sicherheitsgewinn nach dem Umbau zu einem Kreisverkehr profitieren. Französischen Untersuchungen zufolge liegt der Prozentsatz der Unfälle mit Radfahrerbeteiligung an innerörtlichen Kreisverkehren doppelt so hoch wie an sonstigen Knotenpunkten. Aktuelle belgische Untersuchungen deuten sogar darauf hin, dass sich das Risiko für Radfahrer, in einen Unfall schwerem Personenschaden verwickelt zu werden, nach dem Umbau in einen Kreisverkehr erhöht hat.

Die Brilon Bondzio Weiser Ingenieurgesellschaft für Verkehrswesen mbH hat im Auftrag des GDV im Jahre 2012 eine Untersuchung zur Sicherheit innerörtlicher Kreisverkehre [1] vorgelegt. Im Rahmen dieser Untersuchung wurde festgestellt, dass umlaufende Radwege mit Bevorrechtigung der Radfahrer an den Querungsstellen unter Verkehrssicherheitsgesichtspunkten deutlich ungünstiger zu bewerten sind als andere regelkonforme Radverkehrsführungen.

Umlaufende Radwege stellen jedoch eine weit verbreitete Standardführungsform an Kreisverkehren dar und werden aus einem subjektiven Sicherheitsgefühl heraus häufig gefordert. Vor diesem Hintergrund hat der GDV die Brilon Bondzio Weiser Ingenieurgesellschaft für Verkehrswesen $\mathrm{mbH}$ mit einem weiter führenden Forschungsprojekt zur sicheren Führung von Radfahrern auf bevorrechtigten Radwegen an Kreisverkehren beauftragt. Im Rahmen dieses Forschungsprojektes ist zu untersuchen, inwieweit durch die Wahl bestimmter Gestaltungs- und Ausstattungselemente ein möglichst hohes Maß an Verkehrssicherheit für Radfahrer an bevorrechtigten Radwegen erreicht werden kann. 


\section{Literaturübersicht zur Führung von Radwegen an Kreisverkehren}

\section{$2.1 \quad$ Forschungsarbeiten Deutschland}

Alrutz et al. 1992 [2]

Alrutz et al. konzentrierten sich in ihrer Untersuchung auf die Verkehrssicherheit von Radfahrern und Fußgängern an einstreifigen Kreisverkehren. Dabei wurden die Unfalldaten von 13 Kreisverkehren in Nordrhein-Westfalen ausgewertet. Abschließend wurden die folgenden Empfehlungen gegeben:

- Kreisverkehre stellen grundsätzlich auch unter Berücksichtigung der Sicherheit von Radfahrern eine geeignete Lösungsform zur Ausbildung innerörtlicher Knotenpunkte dar.

- Bei konsequent geschwindigkeitsdämpfender Ausbildung des Kreisverkehrs wird der Mischverkehr empfohlen.

- Umlaufende Radwege kommen grundsätzlich auch in Betracht. Sie sollen aber nur um 1 bis $2 \mathrm{~m}$ von der Kreisfahrbahn abgesetzt werden. Nur bei stark belasteten Kreisverkehren ist eine Abrückung der Querungsstellen um bis zu 5 m in Erwägung zu ziehen.

- Radfahrstreifen auf der Kreisfahrbahn werden aufgrund der hohen Konfliktträchtigkeit abgelehnt.

\section{Brilon, Stuwe, Drews 1993 [3]}

Im Rahmen dieser Untersuchung wurden 50 Kreisverkehre hinsichtlich ihrer Verkehrssicherheit untersucht. Die Einteilung nach Kreisverkehrsarten erfolgte nach dem Kriterium Außendurchmesser. Für die 28 kleinen Kreisverkehre (Außendurchmesser maximal $45 \mathrm{~m}$ ) ergaben sich die folgenden Unfallkenngrößen

- Unfallrate: $\quad 1,27$ Unfälle $/ 10^{6} \mathrm{Fz}$

- Unfallkostenrate: $17,89 \mathrm{DM} / 10^{3} \mathrm{Fz}$

Kleine Kreisverkehre schnitten dabei hinsichtlich der Unfallkenngrößen deutlich günstiger ab als große Kreisverkehre.

Erstmalig wurde ein Zusammenhang zwischen dem Unfallgeschehen und der Art der Radverkehrsführung hergestellt. Als sicherste Lösung für Radfahrer stellte sich der Mischverkehr heraus. Am Ungünstigsten schnitt ein Radfahrstreifen im Kreis ab.

Anschließend wurde für 25 überwiegend innerorts gelegene einstreifige Kreisverkehre ein VorherNachher-Vergleich ohne Kontrollgruppe durchgeführt. Nach dem Umbau zum Kreisverkehr sank

- die Unfallrate von 1,74 auf 1,12 Unfälle / $10^{6} \mathrm{Fz}$ und

- die Unfallkostenrate von 33,38 auf $14,03 \mathrm{DM} / 10^{3} \mathrm{Fz}$ 
Von dem Sicherheitsgewinn profitierten insbesondere Kraftfahrer und Fußgänger. Die Unfälle mit Radfahrerbeteiligung nahmen hingegen nach dem Umbau im Mittel zu. Dabei erwiesen sich Radfahrstreifen auf der Kreisfahrbahn als die für Radfahrer mit Abstand unsicherste Verkehrsführung.

\section{Brilon 1997 [4]}

Brilon griff in seiner Veröffentlichung auf die Unfalldaten der Untersuchung von Brilon, Stuwe, Drews [4] sowie auf die Daten von acht weiteren einstreifigen Kreisverkehren zurück. Er kam zu den folgenden Ergebnissen:

- Nach dem Umbau zum Kreisverkehr hatten sich die Unfallkosten an allen Kreisverkehren (innerhalb und außerhalb bebauter Gebiete) um im Mittel $57 \%$ reduziert. Bei Anlagen innerhalb bebauter Gebiete betrug die Reduktion der Unfallkosten nach dem Umbau zum Kreisverkehr gegenüber dem Vorher-Zustand im Mittel $36 \%$.

- Fußgänger verkehrten an Kreisverkehren im Vergleich zu Kreuzungen und Einmündungen besonders sicher

- Radfahrer hatten an Kreisverkehren keine größeren Gefahren zu erwarten als an anderen Verkehrsanlagen. Die Sicherheit hing jedoch im hohen Maße von der Art der Radverkehrsführung ab. Radfahrstreifen auf der Kreisfahrbahn sind dabei unbedingt zu vermeiden. Die Führung im Mischverkehr sowie die Anlage von kreisumlaufenden Radwegen wurden hingegen als sichere Radverkehrsführung bewertet. Bei kreisumlaufenden Radwegen ist darauf zu achten, dass die Querungsstellen nicht weiter als $5 \mathrm{~m}$ vom Kreisrand abgerückt werden.

\section{Haller, Lange, Alrutz, Stellmacher-Hein 2000 [5]}

Im Rahmen der Studie wurde das Unfallgeschehen mit Radfahrer- und Fußgängerbeteiligung an 46 Kreisverkehren untersucht. Darüber hinaus wurden an 16 Kreisverkehren Verhaltensbeobachtungen durchgeführt.

Die Untersuchung kam zu dem Ergebnis, das Kreisverkehre auch für Fußgänger und Radfahrer sichere Verkehrsanlagen sind. Aus Gründen der Vereinheitlichung der Regelungen für Fußgänger wurde grundsätzlich die Anlage von Fußgängerüberwegen empfohlen. Bezogen auf die Führung des Radverkehrs wurden sowohl der Mischverkehr als auch die Anlage kreisumlaufener Radwege als gleichermaßen sicher eingestuft. Bei hohen Verkehrsbelastungen (DTV $>15.000 \mathrm{Kfz} / 24 \mathrm{~h}$ ) sollten aus Gründen der Akzeptanz eher kreisumlaufende Radwege angelegt werden.

\section{Bondzio, Ortlepp, Scheit, Voss 2012 [1]}

Die Untersuchung beschäftigte sich mit der Verkehrssicherheit einstreifiger Kreisverkehre innerorts. In die Untersuchung gingen 100 Kreisverkehre mit insgesamt 1.015 Unfällen ein. Die Kreisverkehre entsprachen hinsichtlich der geometrischen Gestaltung im Wesentlichen den Vorgaben der Regelwerke. Für das Datenkollektiv wurden die folgenden Unfallkenngrößen ermittelt:

- Unfallrate betrug 0,60 Unfälle / $10^{6} \mathrm{Kfz}$

- Unfallkostenrate betrug $6,28 € / 10^{3} \mathrm{Kfz}$ 
An etwa $10 \%$ aller Unfälle waren Radfahrer beteiligt. Bezogen auf die Unfälle mit Personenschäden lag der Anteil der Unfälle mit Radfahrerbeteiligung hingegen bei $28 \%$. Bei Unfällen mit Radfahrerbeteiligung hängt der Unfalltyp stark von der Art der Radverkehrsführung ab. Bei Kreisverkehren mit der Führung der Radfahrer im Mischverkehr ereigneten sich etwa $40 \%$ aller Unfälle bei der Einfahrt in den Kreis (Unfalltyp 303). Weitere $19 \%$ der Unfälle ereigneten sich unmittelbar vor der Ausfahrt von der Kreisfahrbahn (Unfalltyp 232). Bei bevorrechtigten umlaufenden Radwegen ereigneten sich $88 \%$ aller Unfälle mit Radfahrerbeteiligung an den Querungsstellen.

Die Untersuchung kam zu dem Ergebnis, dass die Verkehrssicherheit der Radfahrer von der Führungsform des Radverkehrs beeinflusst wird. Als besonders sicher für Radfahrer erwiesen sich Kreisverkehre ohne Radverkehrsanlagen (Mischverkehr) sowie Kreisverkehre mit umlaufenden Radwegen und vorfahrtrechtlicher Unterordnung der Radfahrer an den Querungsstellen. Bei den Kreisverkehren mit umlaufenden Radwegen und vorfahrtrechtlicher Überordnung der Radfahrer an den Querungsstellen wurde hingegen ein deutlich höheres Unfallrisiko für Radfahrer nachgewiesen.

\section{$2.2 \quad$ Niederlande}

\section{Van Minnen 1995 [6]}

Im Rahmen dieser Studie wurden 177 niederländische Kreisverkehre hinsichtlich ihrer Verkehrssicherheit untersucht. Nach dem Umbau zum Kreisverkehr wurde ein Rückgang der Unfallzahlen um 47\% festgestellt. Die Anzahl der Verunglückten ging sogar um $71 \%$ zurück. Allerdings wurde wiederum festgestellt, dass nicht alle Verkehrsteilnehmer gleichermaßen vom Verkehrssicherheitsgewinn profitierten. Einem Rückgang der Verunglücktenzahlen unter Kfz-Fahrern von 95\% und unter Fußgängern von $89 \%$ stand ein Rückgang der Verunglücktenzahlen bei Radfahrern von 30\% gegenüber.

Im Rahmen der Studie wurden auch verschiedene Radverkehrsführungen an Kreisverkehren untersucht. Die Studie kam auch zu dem Ergebnis, dass ab einer Verkehrsbelastungen von etwa $8.000 \mathrm{Kfz} / 24 \mathrm{~h}$ (Summe des zuführenden Verkehrs) umlaufende Radwege sicherer sind als der Mischverkehr und als Radfahrstreifen auf der Kreisfahrbahn.

\section{Van Minnen 1998 [7]}

Der Schwerpunkt dieser Untersuchung lag auf der Analyse der Verkehrssicherheit von Kreisverkehren mit umlaufenden Radwegen. Dabei wurden 17 Kreisverkehre mit Vorrang und 28 Kreisverkehre ohne Vorrang hinsichtlich ihrer Verkehrssicherheit verglichen. Der Autor kam zu dem Ergebnis, dass sowohl die Unfallzahlen als auch die Anzahl der Verunglückten an Kreisverkehren ohne Vorrang für Radfahrer deutlich günstiger ausfallen als an Kreisverkehren mit Vorrang für Radfahrer.

\section{Weijermars, W. 2001 [8]}

In dieser Untersuchung wurden ebenfalls Kreisverkehre mit Vorrang für Radfahrer (30 Anlagen) mit Kreisverkehren ohne Vorrang für Radfahrer (36 Anlagen) verglichen. Auch in dieser Untersuchung stellten sich die Kreisverkehre ohne Vorrang für Radverkehr als deutlich sicherer heraus, als Kreisverkehre mit Vorrang für den Radverkehr. 


\subsection{Belgien}

\section{Daniels, Nuits, Wets 2008 [9]}

Die Autoren veröffentlichten 2008 die Ergebnisse einer Studie zur Verkehrssicherheit von Radfahrern an 95 Kreisverkehren in Flandern. Von diesen Kreisverkehren lagen 42 Anlagen innerorts und 53 Anlagen außerorts. Die Untersuchung war als Vorher-Nachher-Vergleich mit Kontrollgruppe konzipiert. Es wurden ausschließlich Unfälle mit Personenschäden und Radfahrerbeteiligung ausgewertet.

Die Untersuchung kam zu dem Ergebnis, dass sich die Anzahl der Unfälle mit Radfahrerbeteiligung nach dem Umbau zu einem Kreisverkehr um 29\% erhöht hatte. Bei den Radfahrerunfällen mit Getöteten und Schwerverletzten wurde sogar ein Anstieg um etwa 50\% diagnostiziert. Mit 48\% fiel der Anstieg der Unfälle mit Personenschäden und Radfahrerbeteiligung bei innerörtlichen Kreisverkehren besonders stark aus. Der Anstieg der Unfälle mit Getöteten oder Schwerverletzten betrug innerorts sogar etwa 80\%. Außerorts konnte im Vorher-Nachher-Vergleich hingegen keine statistisch signifikante Veränderung nachgewiesen werden.

Im Rahmen der Studie wurde keine differenzierte Betrachtung des Unfallgeschehens in Abhängigkeit von der Art der Radverkehrsführung vorgenommen. Darüber hinaus wurde die Kreisverkehrsform (einstreifig oder mehrstreifig) nicht berücksichtigt.

\section{Daniels, Brijs, Nuyts, Wets 2009 [10]}

In einer ergänzenden Studie wurde die Abhängigkeit des Unfallgeschehens mit Radfahrerbeteiligung von der Art der Radverkehrsführung untersucht. Die Unfalldaten entstammten im Wesentlichen der Untersuchung von 2008.

Bezogen auf alle Unfälle mit Personenschäden und Radfahrerbeteiligung wurde ein Zusammenhang zwischen der Unfallsituation und der Art der Radverkehrsführung deutlich. Dabei schnitten die Kreisverkehre mit Radfahrstreifen auf der Kreisfahrbahn besonders schlecht ab. Bei allen anderen Führungsformen (Mischverkehr, umlaufende Radwege mit und ohne Vorrang, niveaufreie Querung) wurde hingegen eine Verringerung der Unfallzahlen gegenüber dem vorherigen Knotenpunkt festgestellt.

Bei einer ausschließlichen Betrachtung der Unfälle mit Getöteten und Schwerverletzten ergab sich hingegen eine Erhöhung der Unfallzahlen nach dem Umbau. Hier schnitten die Kreisverkehre mit umlaufenden Radweg besonders schlecht ab. Dieser Zusammenhang konnte aber nicht statistisch abgesichert werden.

Auch bei dieser Untersuchung fand der Kreisverkehrstyp keine Berücksichtigung. 


\subsection{Dänemark}

\section{Hels, Orozova-Bekkevold 2007 [11]}

Im Rahmen dieser Studie wurde der Zusammenhang zwischen Unfällen mit Radfahrerbeteiligung und Kreisverkehrsparametern, wie Geometrie und Verkehrsbelastungen, untersucht. In die Untersuchung wurden 88 Kreisverkehre auf der Insel Fünen einbezogen. Als methodische Besonderheit ist anzumerken, dass die Unfalldaten aus der Notaufnahme des Krankenhauses in Odense stammten. Eine nachträgliche Überprüfung ergab, dass lediglich $25 \%$ der vom Krankenhaus gemeldeten Unfälle mit Radfahrerbeteiligung polizeilich erfasst wurden.

Die folgenden Zusammenhänge konnten nachgewiesen werden:

- Je höher die Radverkehrsstärken, desto höher die Unfallzahlen mit Radfahrerbeteiligung.

- Je älter der Kreisverkehre, desto höher die Unfallzahlen mit Radfahrerbeteiligung.

- Je höher die mögliche Geschwindigkeit für Kraftfahrer im Kreis, desto höher die Unfallzahlen mit Radfahrerbeteiligung.

Ein Zusammenhang zwischen dem Unfallgeschehen und der Art der Radverkehrsführung konnte nicht nachgewiesen werden.

\section{Jensen 2015 [12]}

Jensen führte eine Vorher-Nachher-Untersuchung zur Entwicklung der Verkehrssicherheit nach der Umgestaltung von 255 Knotenpunkten in einstreifige Kreisverkehre in Dänemark durch. Hinsichtlich der Verkehrssicherheit von Radfahrern kam er zu den folgenden Ergebnissen:

- Es besteht ein Zusammenhang zwischen der Größe der Kreisinsel und der Verkehrssicherheit für Radfahrer. Dabei zeigen Kreisverkehre mit einem Durchmesser der Kreisinsel einschließlich des Innenrings von 20 bis $40 \mathrm{~m}$ das höchste Verkehrssicherheitsniveau.

- Ebenso wurde ein positiver Einfluss der Überhöhung der Kreisinsel auf die Verkehrssicherheit von Radfahrern festgestellt. Kreisverkehre mit hoher Kreisinsel (Kreismittelpunkt mehr als $2 \mathrm{~m}$ über der Kreisfahrbahn) sind für Radfahrer deutlich sicherer als Kreisverkehre mit niedrigerer Kreisinsel.

- Als sicherste Führungsform erwies die Anlage umlaufender mit vorfahrtrechtlicher Unterordnung des Radverkehrs. Radfahrstreifen auf der Kreisfahrbahn stellten sich mit Abstand als die für Radfahrer unsicherste Führungsform heraus. 


\subsection{Schweden}

\section{Brüde, Larsson 2000 [13]}

Die Autoren berichteten über eine Studie an 72 Kreisverkehren in Schweden. Dabei wurden die Erkenntnisse aus anderen europäischen Staaten bestätigt, dass das Unfallrisiko in Kreisverkehren von der Verkehrsbelastung und der Geschwindigkeit abhängt. Bezogen auf die Verkehrssicherheit von Radfahrern sprachen sich die Autoren für die Anlage umlaufender Radwege aus. Bei der Führung im Mischverkehr wurde ein um 2,5 fach höheres Unfallrisiko für Radfahrer ermittelt.

\section{Sakshaug, Laureshyn, Svensson, Hydén 2010 [14]}

Die Autoren veröffentlichten 2010 eine Studie zum Interaktionsverhalten zwischen Kraftfahrern und Radfahrern an Kreisverkehren. Neben einer Unfallanalyse an 15 Kreisverkehren (9 mit umlaufenden Radwegen und 6 mit Mischverkehr) wurden an zwei Kreisverkehren Verhaltensbeobachtungen durchgeführt.

Im Rahmen der Untersuchung wurden unterschiedliche Konflikttypen mit der Wahrscheinlichkeit des Auftretens bestimmt. Darüber hinaus wurde auch das Verhalten der Verkehrsteilnehmer im Konfliktfall analysiert.

An Kreisverkehren mit umlaufenden Radwegen waren an $68 \%$ aller Unfälle mit Radfahrerbeteiligung Radfahrer beteiligt, die den Radweg entgegen der vorgeschriebenen Fahrtrichtung befuhren. An Kreisverkehren mit Mischverkehr ereigneten sich 73\% aller Unfälle mit Radfahrerbeteiligung durch Vorfahrtmissachtungen bevorrechtigter Radfahrer durch wartepflichtige Kraftfahrer bei der Einfahrt in den Kreisverkehr. Diese Konfliktsituation wurde auch in den Verhaltensbeobachtungen als besonders gefährlich klassifiziert.

\subsection{Zusammenfassung und Schlussfolgerungen}

Hinsichtlich der Verkehrssicherheit der Radfahrer kommen die einzelnen Studien zu unterschiedlichen Ergebnissen. Übereinstimmend kann festgestellt werden, dass Radfahrer weniger stark vom Sicherheitsgewinn profitieren als andere Verkehrsteilnehmer.

Die Untersuchungen kommen übereinstimmend zu dem Ergebnis, dass Radfahrstreifen auf der Kreisfahrbahn die mit Abstand unsicherste Führungsform für den Radverkehr darstellen. Es besteht auch Konsens, dass umlaufende Radwege mit vorfahrtrechtlicher Unterordnung der Radfahrer an den Querungsstellen die für Radfahrer sicherste Führungsform sind.

Ein Zusammenhang zwischen geometrischen Kreisverkehrsparametern und der Verkehrssicherheit für Radfahrer sind mit Ausnahme der Untersuchung von Jensen [12] in keiner Untersuchung nachgewiesen. Hier besteht erheblicher Forschungsbedarf. 


\section{Regelwerke}

\subsection{Deutschland}

\section{Merkblatt für die Anlage von Kreisverkehren 2006 [15]}

Das aktuelle Regelwerk der Forschungsgesellschaft für Straßen- und Verkehrswesen beschäftigt sich neben einstreifigen Kreisverkehren auch mit Minikreisverkehren sowie mit mehrstreifigen Kreisverkehren. Die grundsätzlichen Gestaltungsprinzipien der vorherigen Regelwerke werden nach wie vor übernommen. Im Detail werden jedoch Änderungen deutlich.

Gemäß Merkblatt für die Anlage von Kreisverkehren [29] ist die Führung der Radfahrer im Mischverkehr bei Verkehrsbelastungen bis zu 15.000 Kfz / 24h (Summe des zuführenden Verkehrs) zu empfehlen. Bei höheren Verkehrsbelastungen weichen Radfahrer zunehmend auf die Gehwege aus. Durch einen baulich angelegten und mit einem Bord abgesetzten Innenring soll das Überholen von Radfahrern durch Kraftfahrer weitgehend unterbunden werden. Gemäß Merkblatt besteht grundsätzlich auch die Möglichkeit in den Knotenpunktarmen durchlaufende Radwege am Kreisverkehr zu unterbrechen und den Radverkehr auf der Kreisfahrbahn zu führen. In diesen Fällen wird allerdings empfohlen, zu prüfen, ob die Gehwege zusätzlich zur Mitbenutzung durch Radfahrer frei gegeben werden können.

Die Führung auf umlaufenden Radwegen kommt insbesondere dann in Betracht, wenn in den Knotenpunktarmen baulich angelegte Radwege vorhanden sind. An den Querungsstellen ist der Radverkehr in der Regel bevorrechtigt. Sofern Fußgängerüberwege angelegt sind, ist die Bevorrechtigung des Radverkehrs zwingend erforderlich. An den Querungsstellen sollen die bevorrechtigten Furten nach Möglichkeit um 4,00 bis 5,00 m von der Kreisfahrbahn abgesetzt werden.

Die vorfahrtrechtliche Unterordnung der Radfahrer an den Querungsstellen wird innerhalb bebauter Gebiete nicht grundsätzlich ausgeschlossen. Für Kreisverkehre außerhalb bebauter Gebiete ist die Unterordnung die Standardlösung. Die Querungsstellen sollen dann um 5,00 m von der Kreisfahrbahn abgesetzt werden. Die Unterordnung ist durch verkleinerte Zeichen 205 StVO anzuzeigen.

Als der zentrale Gestaltungsgrundsatz zur Gewährleistung einer ausreichenden Verkehrssicherheit wird die Ablenkung geradeaus fahrender Kraftfahrer durch die Kreisinsel hervorgehoben. Die Ablenkung soll das Zweifache der Fahrstreifenbreite der Knotenpunktzufahrten nicht unterschreiten. Sofern ein Innenring angelegt ist, wird die dadurch erzielte Ablenkung in die Berechnung des Ablenkmaßes einbezogen. 
Die folgende Abbildung verdeutlicht das Prinzip der Ablenkung.

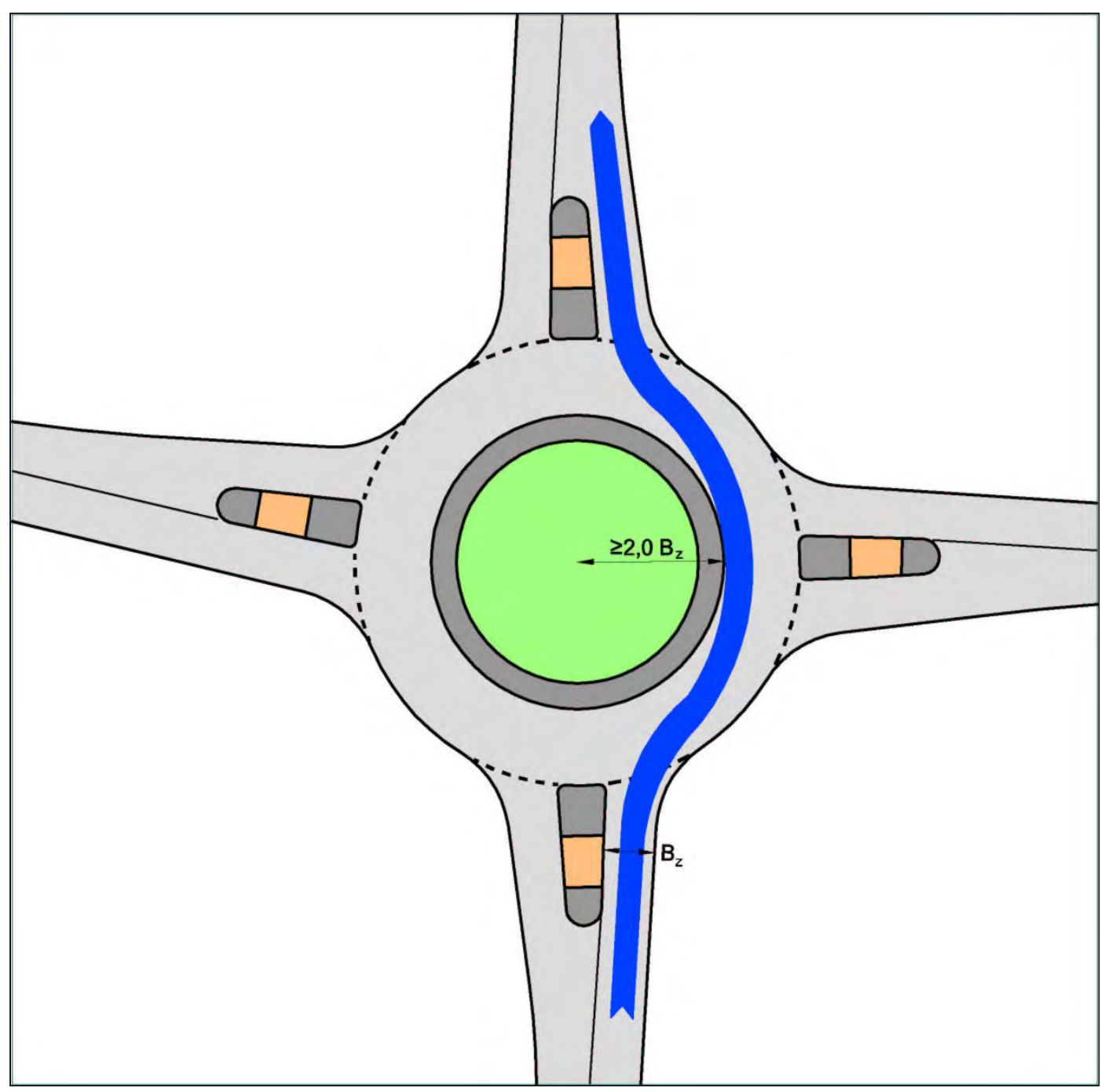

Abbildung 1: Ablenkung geradeausfahrender Kraftfahrzeuge [Eigene Darstellung nach Vorlage [29]]

Die Darstellung verdeutlicht auch, dass eine ausreichende Ablenkung nur bei richtiger Platzierung der Kreisinsel erreicht werden kann. Idealerweise ist der Mittelpunkt der Kreisinsel in den Schnittpunkt der Achsen der Knotenpunktarme zu legen.

\subsection{Schweiz}

\section{Schweizer Norm 640252 Knoten Führung des leichten Zweiradverkehrs [16]}

In der Schweizer Norm 640252 wird unter anderem die Führung des Radverkehrs an Kreisverkehren geregelt. Der Mischverkehr stellt dabei an einstreifigen Kreisverkehren die Standardführungsform dar. Umlaufende Einrichtungsradwege werden nur bei erheblichen Verkehrsmengen sowie bei großen oder mehrstreifigen Kreisverkehren empfohlen. Die Querungsstellen sollen dann um $5 \mathrm{~m}$ bis $7 \mathrm{~m}$ vom Kreisrand abgesetzt werden. Der Radverkehr ist gegenüber dem Kfz-Verkehr vorfahrtrechtlich unterzuordnen. 


\section{3 Österreich}

\section{RVS 03.05.14 Plangleiche Knotenpunkte - Kreisverkehre [17]}

Die Österreichische Richtlinie aus dem Jahr 2010 empfiehlt, die Radverkehrsführung um einen Kreisverkehr in Abhängigkeit von der Führung vor und hinter dem Kreisverkehr zu wählen. Sofern vor und hinter dem Kreisverkehr bauliche getrennte Radverkehrsanlagen vorhanden sind, sollten auch um den Kreisverkehr separate Radwege angelegt werden.

Sofern umlaufende Radwege angelegt sind, soll der Abstand der Querungsstelle vom Außenrand der Kreisfahrban eine PKW-Länge $(6 \mathrm{~m})$ betragen.

\section{RVS 03.02.13 Radverkehr [18]}

Diese Richtlinie aus dem Jahr 2014 enthält auch für Kreisverkehre ergänzende Hinweise zur Gestaltung von Radverkehrsanlagen. Bei Kreisverkehren mit einem Außendurchmesser von bis zu $30 \mathrm{~m}$ wird grundsätzlich der Mischverkehr als sicherste Führungsmöglichkeit empfohlen.

Einrichtungsradwege um den Kreisverkehr werden als problematisch angesehen, da mit Befahren in beiden Richtungen zu rechnen ist.

Getrennte kreisumlaufende Radwege werden nur bei Zweirichtungsradwegen und bei großen Kreisverkehren mit hohen Verkehrsbelastungen, insbesondere bei mehrstreifigen Kreisverkehren, empfohlen.

\subsection{Niederlande}

\section{Eenheid in rotondes [19]}

Die niederländische Kreisverkehrsrichtlinie aus dem Jahr 1998 sieht die folgenden Führungsformen für Radverkehr vor:

- Mischverkehr

Diese Führungsform wird bis zu einem Verkehrsaufkommen von $6.000 \mathrm{Kfz} / 24 \mathrm{~h}$ empfohlen.

- Radfahrstreifen auf der Kreisfahrbahn

Diese Führungsform ist grundsätzlich möglich. Allerdings wird darauf hingewiesen, dass hierdurch eine besonders breite Kreisfahrbahn mit den damit verbundenen Nachteilen entsteht. Es wird auf die Möglichkeit der abschnittsweisen baulichen Trennung des Radfahrstreifens von der Kreisfahrban hingewiesen. 
- Separate Radwege mit Vorrang für die Radfahrer

Es wird empfohlen, den Radweg kreisrund mit einem Abstand von etwa $5 \mathrm{~m}$ von der Kreisfahrbahn zu führen. Die Querungsstelle soll ebenfalls um etwa $5 \mathrm{~m}$ vom Kreisrand abgesetzt werden.

Der Verzweigungspunkt des Radwegs in der Ausfahrt soll stärker von der Ausfahrt abgesetzt werden. Die Gründe hierfür sind der höhere Fahrkomfort für Radfahrer sowie der positive Einfluss auf die Leistungsfähigkeit der Kreisverkehrsausfahrt.

Um den Vorrang der Radfahrer zu verdeutlichen wird auf die Möglichkeit hingewiesen, die Querungsstelle um etwa 3 bis $4 \mathrm{~cm}$ gegenüber der Fahrbahn anzuheben.

- Separate Radwege ohne Vorrang für die Radfahrer

Zur Verdeutlichung der Wartepflicht der Radfahrer wird empfohlen, den Radweg enger anliegend mit einem Abstand von etwa $3 \mathrm{~m}$ von der Kreisfahrbahn zu führen. Die Querungsstellen sollen um etwa $10 \mathrm{~m}$ vom Kreisrand abgesetzt werden.

\section{Fietsvorsteken op rotondes [20]}

Im Jahr 2002 wurde eine Ergänzung zur Richtlinie „Eenheit in rotondes“ speziell zur Führung des Radverkehrs herausgegeben. Darin werden die folgenden Empfehlungen gegeben:

- Bei bevorrechtigten Radfahrerfurten wird in den Zu- und Ausfahrten eine zusätzliche dreieckige Blockmarkierung „Haifischzähne“ empfohlen. Darüber hinaus wird die Roteinfärbung empfohlen.

- In Zusammenhang mit Fußgängerüberwegen sollte dem Radfahrer Vorrang eingeräumt werden.

- Bei vorfahrtrechtlicher Unterordnung des Radverkehrs wird die Markierung von Haifischzähnen auf den Radwegen unmittelbar vor der Querung empfohlen. Die Markierung soll für alle Verkehrsteilnehmer gut erkennbar sein.

- Die Trassierung des Radweges um den Kreisverkehr herum soll in Abhängigkeit von der Vorfahrtregelung an den Querungsstellen erfolgen. Bei vorfahrtrechtlicher Überordnung des Radverkehrs soll der Radweg kreisrund um die Kreisfahrbahn geführt werden. Bei vorfahrtrechtlicher Unterordnung ist eine gebogene Führung zu empfehlen.

- Bei Zweirichtungsradwegen wird in den Zufahrten die Anordnung von Zusatzzeichen empfohlen, die auf den Zweirichtungsverkehr hinweisen. 


\section{$4 \quad$ Auswahl der Kreisverkehrsarme}

\subsection{Vorgehensweise}

Im Rahmen der Untersuchung wurde das Unfallgeschehen mit Radfahrerbeteiligung an insgesamt 294 Kreisverkehrsarmen analysiert. Die Analyse erfolgte auf der Grundlage der polizeilichen Unfallprotokolle der Jahre 2011 bis 2013. Dabei wurden die folgenden Arbeitsschritte durchgeführt:

- Auswahl geeigneter Kreisverkehrsarme

- Datenerhebung (Knotenpunktdaten, Daten zur Verkehrsnachfrage, Unfalldaten)

- Analyse der Struktur der Radverkehrsunfälle an den 294 Kreisverkehrsarmen

- Bewertung der Verkehrssicherheit anhand der Unfallkenngrößen

- Untersuchung von Zusammenhängen zwischen den Unfallkenngrößen und der Knotenpunktgeometrie

\subsection{Auswahl der Knotenpunkte}

Es erfolgte eine systematische schriftliche Abfrage bei den folgenden Straßenbaulastträgern hinsichtlich geeigneter Kreisverkehre.

- Bundesweit alle Niederlassungen der Landesstraßenbauverwaltungen

- Alle Kreisbauverwaltungen der Länder Hessen, Niedersachsen und Nordrhein-Westfalen

- Alle kommunalen Bauverwaltungen der Städte über 20.000 Einwohner in den Ländern Hessen, Niedersachsen und Nordrhein-Westfalen

Die Rücklaufquote lag bei etwa $30 \%$. Dabei wurden von den Straßenbaulastträgern 220 Kreisverkehre genannt. Die weitere Analyse der Kreisverkehre zeigte, dass lediglich 32 der genannten Kreisverkehre aufgrund ihrer Geometrie für das Forschungsprojekt geeignet waren.

Dem Forschungsnehmer liegt eine Datenbank mit mehreren hundert Kreisverkehren vor, die überwiegend in Nordrhein-Westfalen verortet sind. Aus dieser Datenbank wurden weitere 71 Kreisverkehre ausgewählt, die den Kriterien

- Führung des Radverkehrs auf umlaufenden Radwegen mit Bevorrechtigung an den Querungsstellen

- Weitest gehend regelkonforme Gestaltung gemäß Merkblatt 2006 [15]

entsprachen.

Insgesamt lagen nach Abschluss der Recherche 103 grundsätzlich geeignete Kreisverkehre mit insgesamt 367 geeigneten Kreisverkehrsarmen vor. In einem weiteren Auswahlprozess wurden daraus 84 Kreisverkehre mit insgesamt 294 Kreisverkehrsarmen ausgewählt, die für die weiteren Untersuchungen betrachtet wurden. 


\subsection{Merkmale der ausgewählten Knotenpunkte}

\subsubsection{Radwegeführung über die Kreisverkehrsarme}

Die einzelnen Kreisverkehrsarme weisen hinsichtlich der Radwegeführung die folgenden Eigenschaften auf:

- Alle Kreisverkehrsarme werden von separaten Radfahrerfurten sowie von benachbarten Fußgängerüberwegen gequert.

- Bei 197 Kreisverkehrsarmen (67 \%) sind Radwege vorhanden. An 89 Kreisverkehrsarmen (30 \%) werden in den Nebenanlagen gemeinsame Geh-/Radwege geführt, die an der Querungsstelle getrennt werden. An 8 Kreisverkehrsarmen werden die Gehwege für den Radverkehr frei gegeben. Die Querung erfolgt auf separaten Furten.

- Im Zuge von 87 Kreisverkehrsarmen (30 \%) ist die Querung für Radfahrer im Zweirichtungsverkehr zugelassen.

- An 69 Kreisverkehrsarmen (23\%) werden die Radfahrer im Zuge der Knotenpunktarme auf der Fahrbahn geführt und ausschließlich um den Kreisverkehr auf umlaufenden Radwegen geführt.

- Etwa 50 \% der Radfahrerfurten sind eingefärbt.

- An 134 Kreisverkehrsarmen (46 \%) sind Piktogramme vorhanden, an 64 Kreisverkehrsarmen ist zusätzlich die Fahrtrichtung angezeigt.

- An 8 Kreisverkehrsarmen sind die Furten aufgepflastert, bzw. durch Plateaus erhöht.

- An etwa der Hälfte der Kreisverkehrsarme (49 \%) sind die Radfahrerfurten beidseitig markiert.

- An 12 Kreisverkehrsarmen sind an den Furten zusätzlich Wartelinien für Kraftfahrer markiert.

\subsubsection{Verkehrsbelastungen $\mathrm{Kfz}$}

Bei der Auswahl der Kreisverkehrsarme wurde besonderer Wert darauf gelegt, insbesondere mittel und höher belastete Kreisverkehre zu berücksichtigen. Die Verkehrsbelastungen der einzelnen Kreisverkehrsarme reichen von unter $1.000 \mathrm{Kfz} / 24 \mathrm{~h}$ bis über $22.000 \mathrm{Kfz} / 24 \mathrm{~h}$. Die folgende Abbildung zeigt die Verkehrsbelastungen an allen untersuchten Kreisverkehrsarmen. 


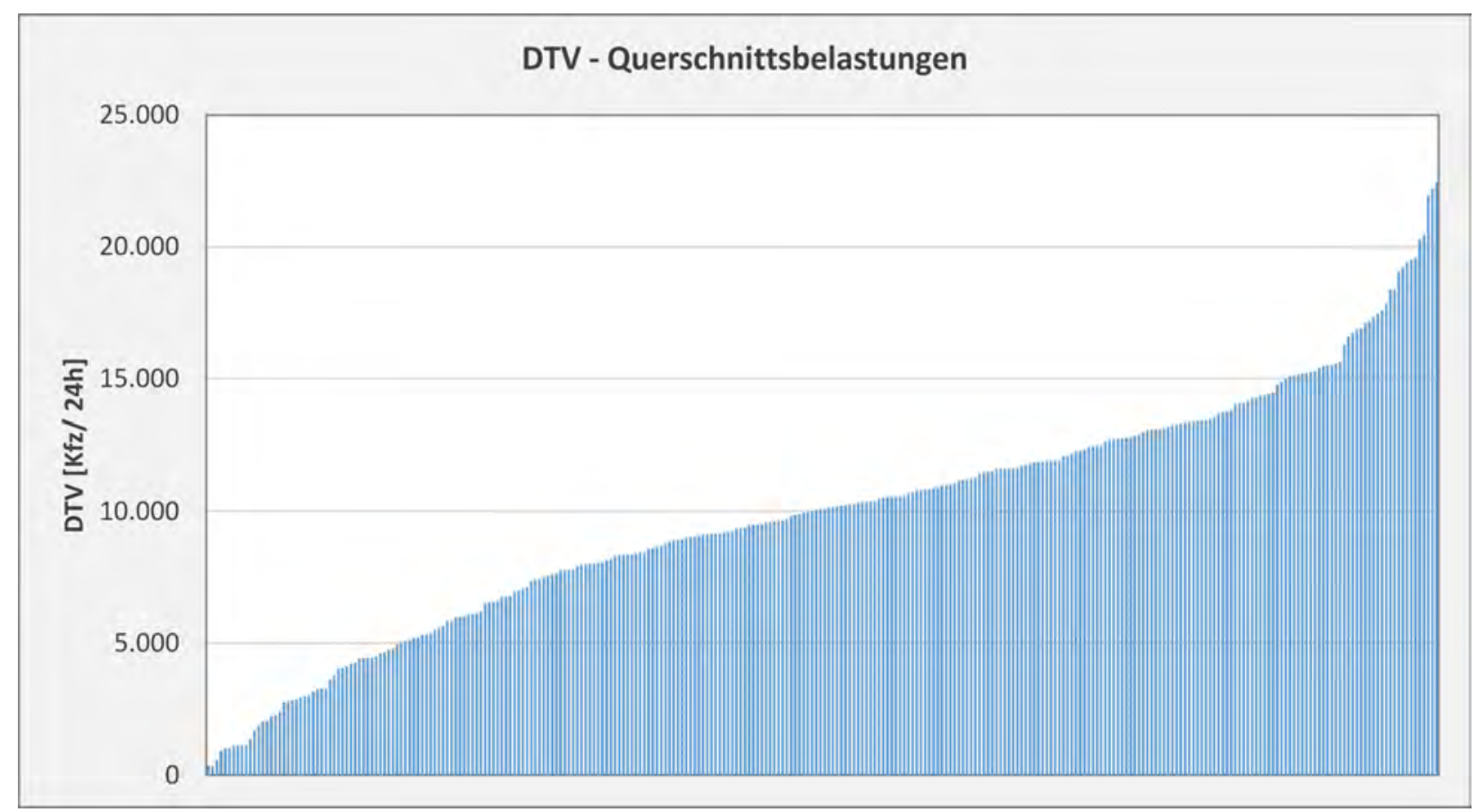

Abbildung 2: Kfz Verkehrsbelastungen im Querschnitt der Kreisverkehrsarme in $\mathrm{Kfz} / 24 \mathrm{~h}$

Die folgende Abbildung zeigt die Verteilung der Kreisverkehre nach Belastungsklassen. Der Schwerpunkt liegt mit 222 Kreisverkehrsarmen (76 \%) im mittleren bis höheren Belastungsbereich zwischen 6.000 $\mathrm{Kfz} / 24 \mathrm{~h}$ und $18.000 \mathrm{Kfz} / 24 \mathrm{~h}$. Etwa $20 \%$ der Kreisverkehrsarmen sind mit unter $6.000 \mathrm{Kfz} / 24 \mathrm{~h}$ im Querschnitt schwächer belastet und lediglich $4 \%$ der Kreisverkehrsarme sind mit über $18.000 \mathrm{Kfz} / 24 \mathrm{~h}$ sehr hoch belastet.

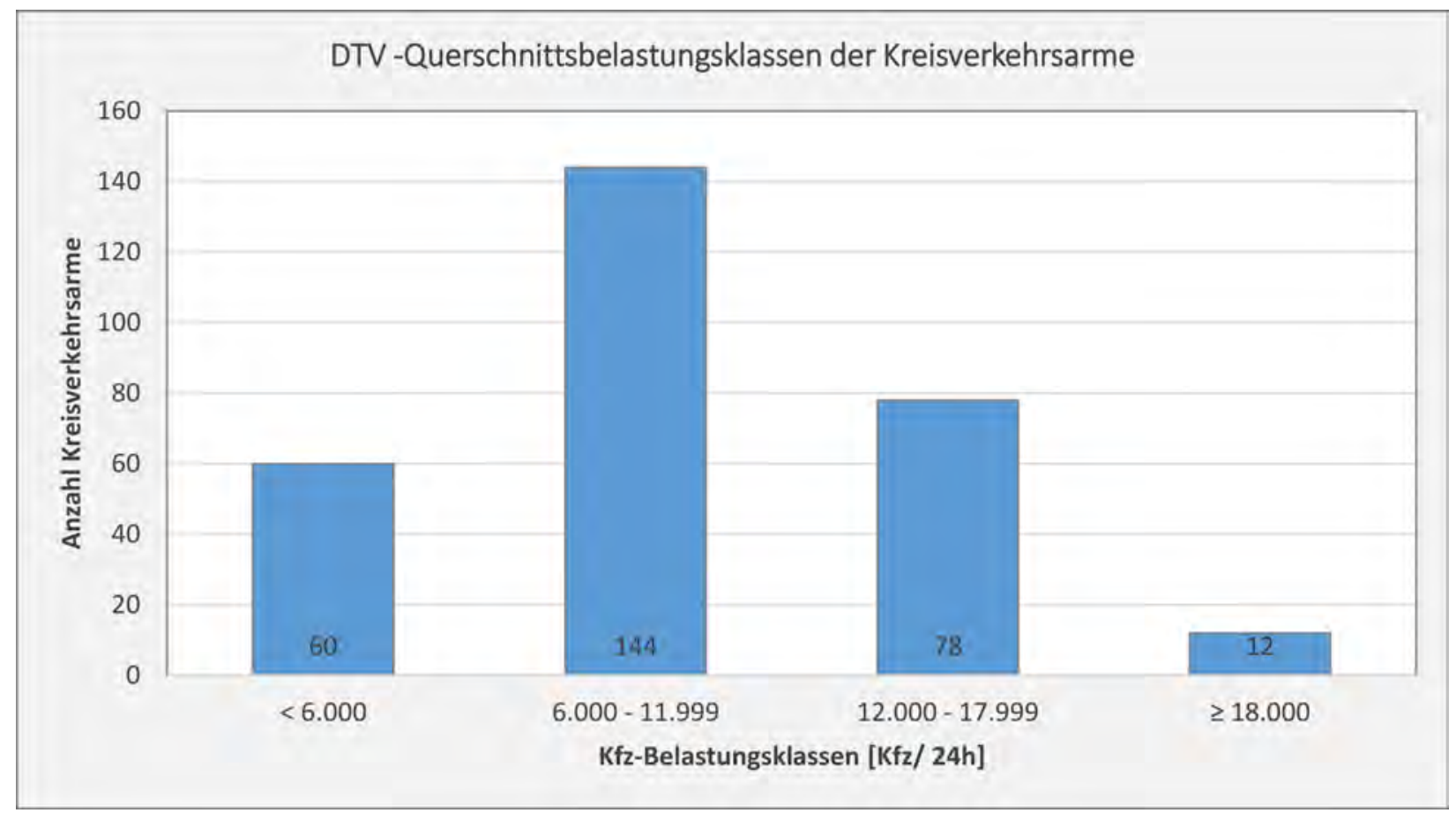

Abbildung 3: Kreisverkehrsarme nach Kfz-Belastungsklassen (Kfz/24h) 


\subsubsection{Verkehrsbelastungen Radfahrer}

Die folgende Abbildung zeigt die Querungen durch Radfahrer an den untersuchten Kreisverkehrsarmen pro $24 \mathrm{~h}$. Die meisten Kreisverkehre weisen unter 500 Radfahrer pro $24 \mathrm{~h}$ auf. An drei Knotenpunktarmen wurden über 2.000 Radfahrer pro $24 \mathrm{~h}$ gezählt.

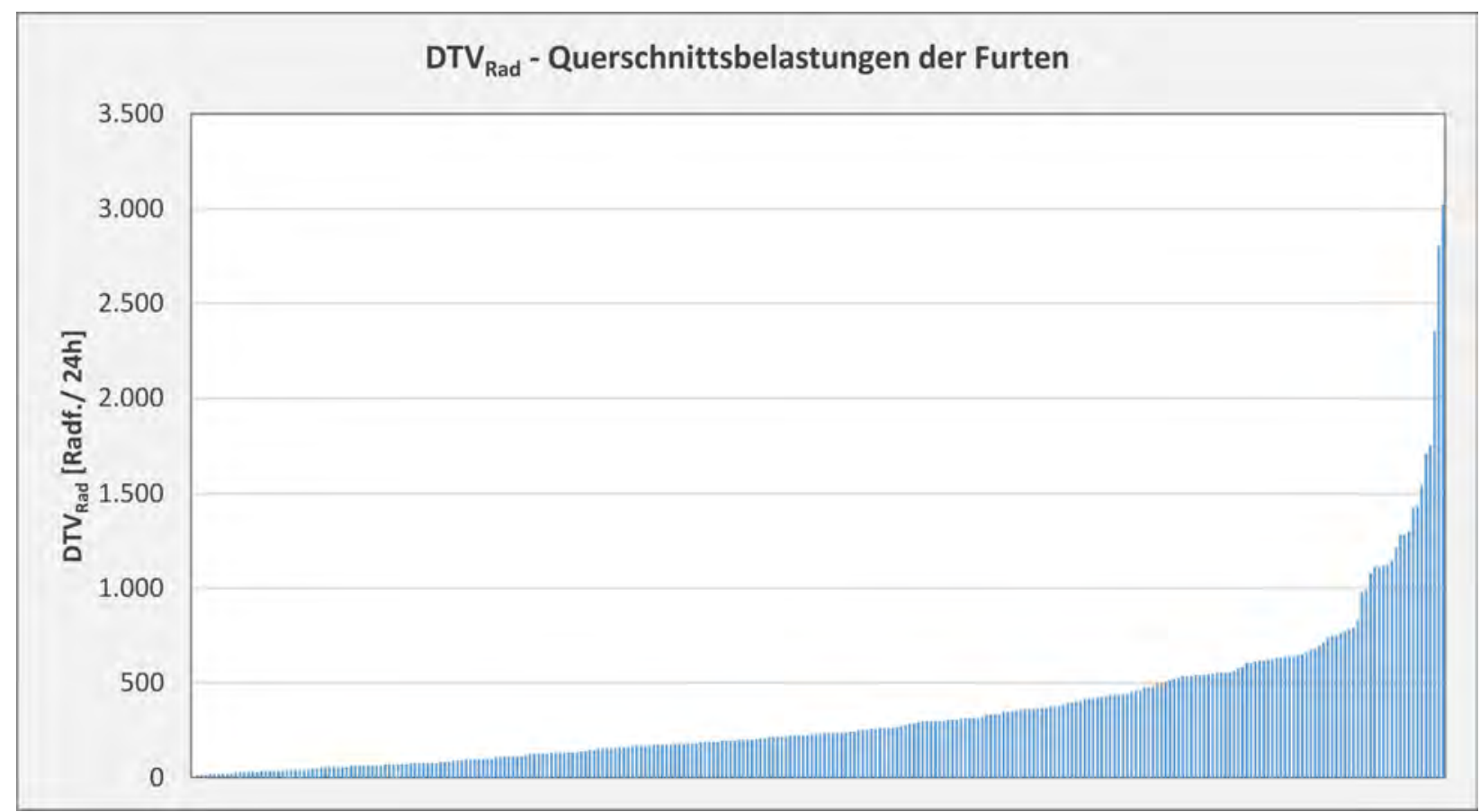

Abbildung 4: Radfahrerquerungen in Rad / 24h

Die folgende Abbildung zeigt die Verteilung der Kreisverkehrsarme nach Belastungsklassen.

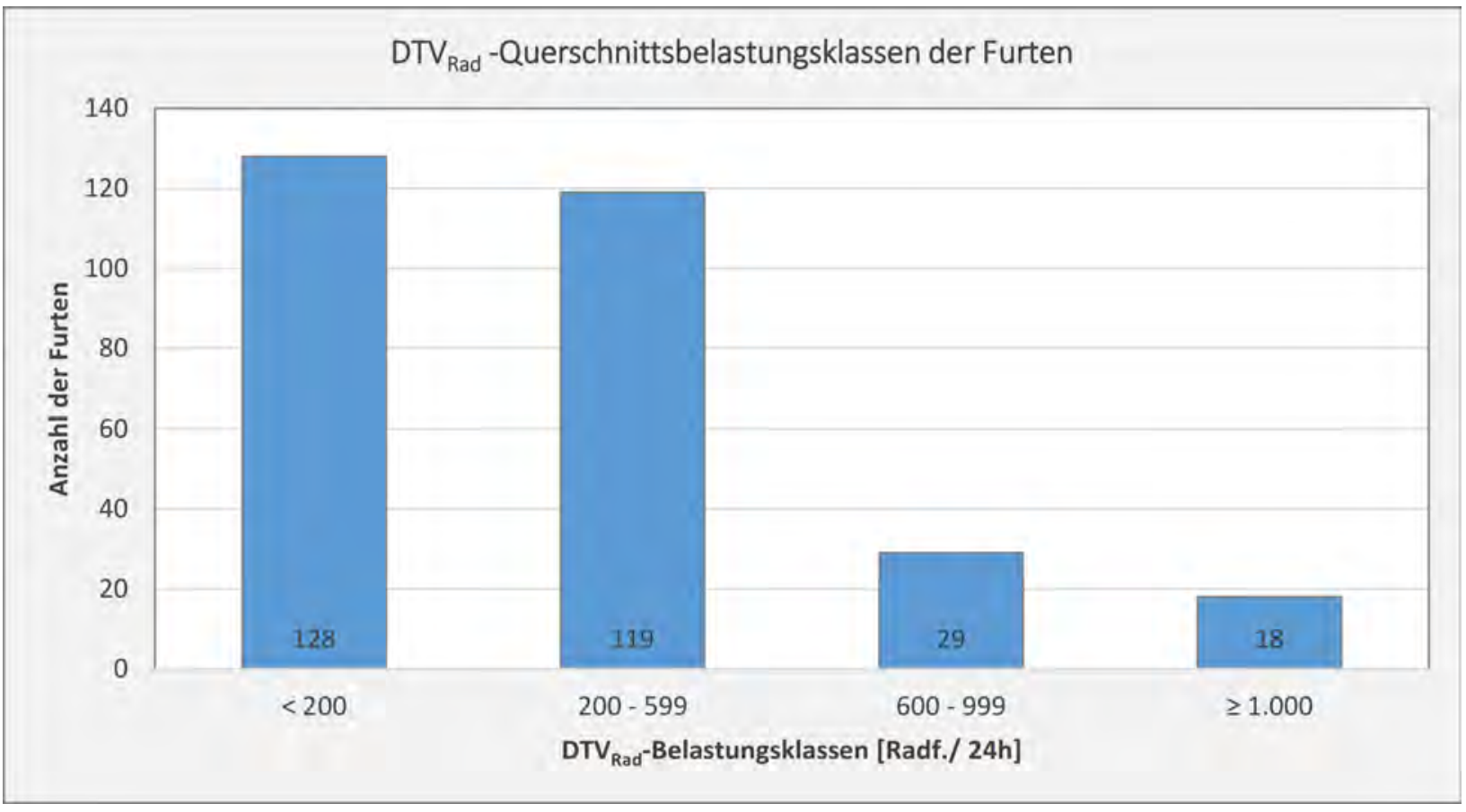

Abbildung 5: Kreisverkehre nach Radfahrer-Belastungsklassen (Rad/24h) 


\subsection{Datenerhebung}

Im Rahmen der Datenerhebung wurden die folgenden Arbeitsschritte durchgeführt:

- Kontaktaufnahme mit Baulastträger

Es wurde jeweils Kontakt mit den Baulastträgern aufgenommen. Dabei wurden für die Untersuchung relevante Daten zur Knotenpunktgeometrie und zur Verkehrsfreigabe abgefragt. In der Regel wurde vom Baulastträger ein maßstabsgerechter Ausbauplan zur Verfügung gestellt. Sofern hinreichend aktuelle Verkehrsnachfragedaten vorlagen, wurden diese ebenfalls vom Baulastträger zur Verfügung gestellt.

- $\quad$ Ortsbesichtigung mit Feststellung der Lage- und Umfeldparameter

Im Rahmen von Ortsbesichtigungen wurden Lage- und Umfeldparameter erhoben. Darüber hinaus wurde abschließend die Eignung der Kreisverkehre für die Untersuchung bewertet. Dabei wurde für jeden Kreisverkehrsarm ein Erfassungsbogen ausgefüllt. Dieser ist in der Anlage 4.1 dokumentiert.

- Zusammenstellung der Unfalldaten

Für die ausgewählten Kreisverkehrsarme wurden die polizeilichen Unfallprotokolle der Radfahrerunfälle der Jahre 2011 bis 2013 besorgt.

- $\quad$ Erhebung der geometrischen Parameter

Die maßgebenden geometrischen Parameter wurden anhand der von den Straßenbaulastträgern zur Verfügung gestellten Planunterlagen ermittelt. Sofern in Ausnahmefällen keine Planunterlagen zur Verfügung gestellt wurden, erfolgte die Ermittlung anhand von Luftbildern, bzw. eine Messung vor Ort.

- Zusammenstellung der Verkehrsnachfragedaten

An denjenigen Knotenpunkten, für die von Seiten der Straßenbaulastträger keine hinreichend aktuellen Verkehrsnachfragedaten zur Verfügung gestellt werden konnten, wurden Verkehrszählungen durchgeführt. 


\section{$5 \quad$ Analyse der Unfallstruktur}

\section{$5.1 \quad$ Unfallkategorien}

Insgesamt wurden im Zeitraum von 2011 bis 2013 an den 294 Kreisverkehrsarmen 224 Unfälle mit Radfahrerbeteiligung polizeilich registriert.

\section{Unfallschwere}

Die Unfallschwere wird durch die folgenden 6 Unfallkategorien beschrieben:

- Unfallkategorie 1: Unfall mit Getötetem

- Unfallkategorie 2: Unfall mit Schwerverletzten

- Unfallkategorie 3: Unfall mit Leichtverletzten

- Unfallkategorie 4: Schwerwiegender Unfall mit Sachschaden

- Unfallkategorie 5: Sonstiger Sachschadensunfall

- Unfallkategorie 6: Sonstiger Sachschadensunfall mit Alkoholeinwirkung

In der folgenden Abbildung ist die Aufteilung der Unfälle auf die Unfallkategorien dargestellt. Dabei zeigt sich, dass es sich bei etwa $75 \%$ der Unfälle mit Leichtverletzten (Unfallkategorie 3) handelt. Bei etwa 5 $\%$ der Unfälle wurden Personen schwer verletzt (Unfallkategorie 2). Fast $20 \%$ der Unfälle sind der Kategorie 5 (sonstiger Sachschaden) zuzuordnen.

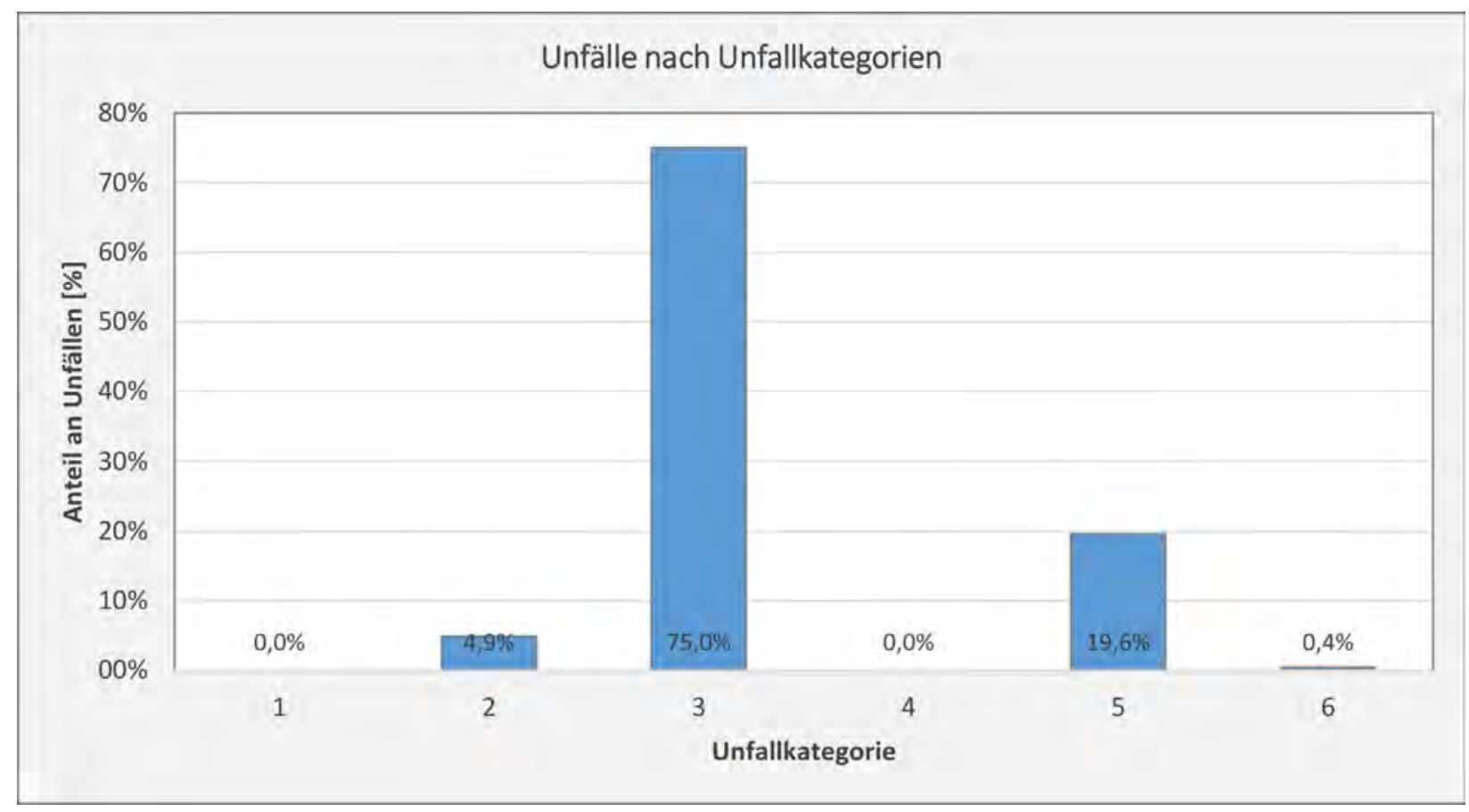

Abbildung 6: Unfälle nach Unfallkategorien 


\section{Verunglückte}

Bei 179 Radfahrerunfällen verunglückten Verkehrsteilnehmer. Dabei wurden $94 \%$ leicht und 6 \% schwer verletzt. Es wurde kein Unfall mit Todesfolge registriert.

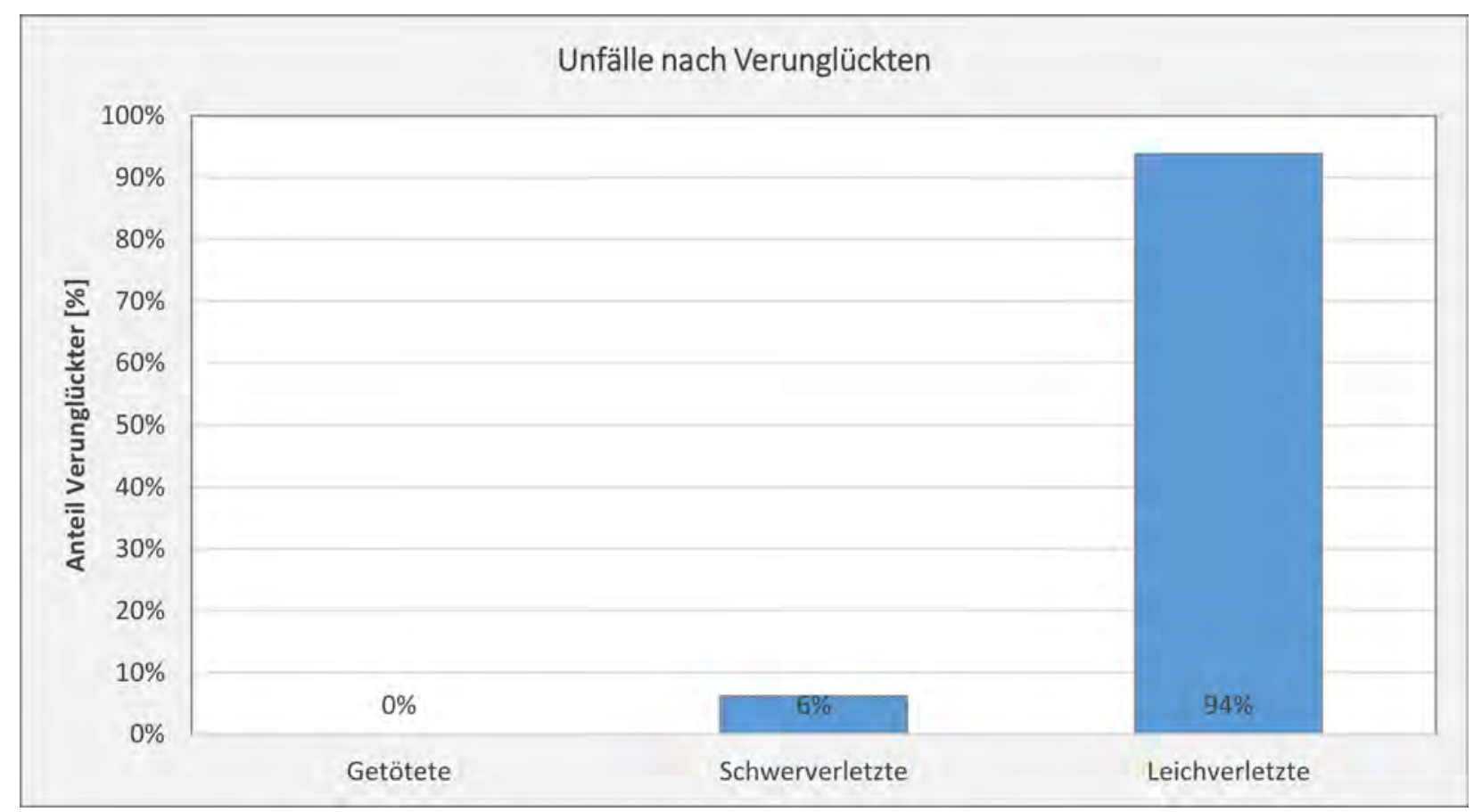

Abbildung 7: Unfälle nach Verunglückten

\section{$5.2 \quad$ Unfalltypen}

Der Unfalltyp kennzeichnet den Verkehrsvorgang bzw. die Konfliktsituation woraus der Unfall entstanden ist. Es werden die folgenden sieben Unfalltypen unterschieden:

- Unfalltyp 1: Fahrunfall

- Unfalltyp 2: Abbiege-Unfall

- Unfalltyp 3: Einbiegen/Kreuzen-Unfall

- Unfalltyp 4: Überschreiten-Unfall

- Unfalltyp 5: Unfall durch ruhenden Verkehr

- Unfalltyp 6: Unfall im Längsverkehr

- Unfalltyp 7: Sonstiger Unfall

Die folgende Abbildung zeigt die Verteilung der Unfälle auf die Unfalltypen. Dabei wird deutlich, dass der Unfalltyp 3 mit insgesamt $54 \%$ aller Unfälle den häufigsten Unfalltyp darstellt. Von großer Bedeutung 
sind zudem die Abbiegeunfälle (Unfalltyp 2) mit $38 \%$ aller Unfälle. Die übrigen Unfalltypen spielen dagegen nur eine untergeordnete Rolle.

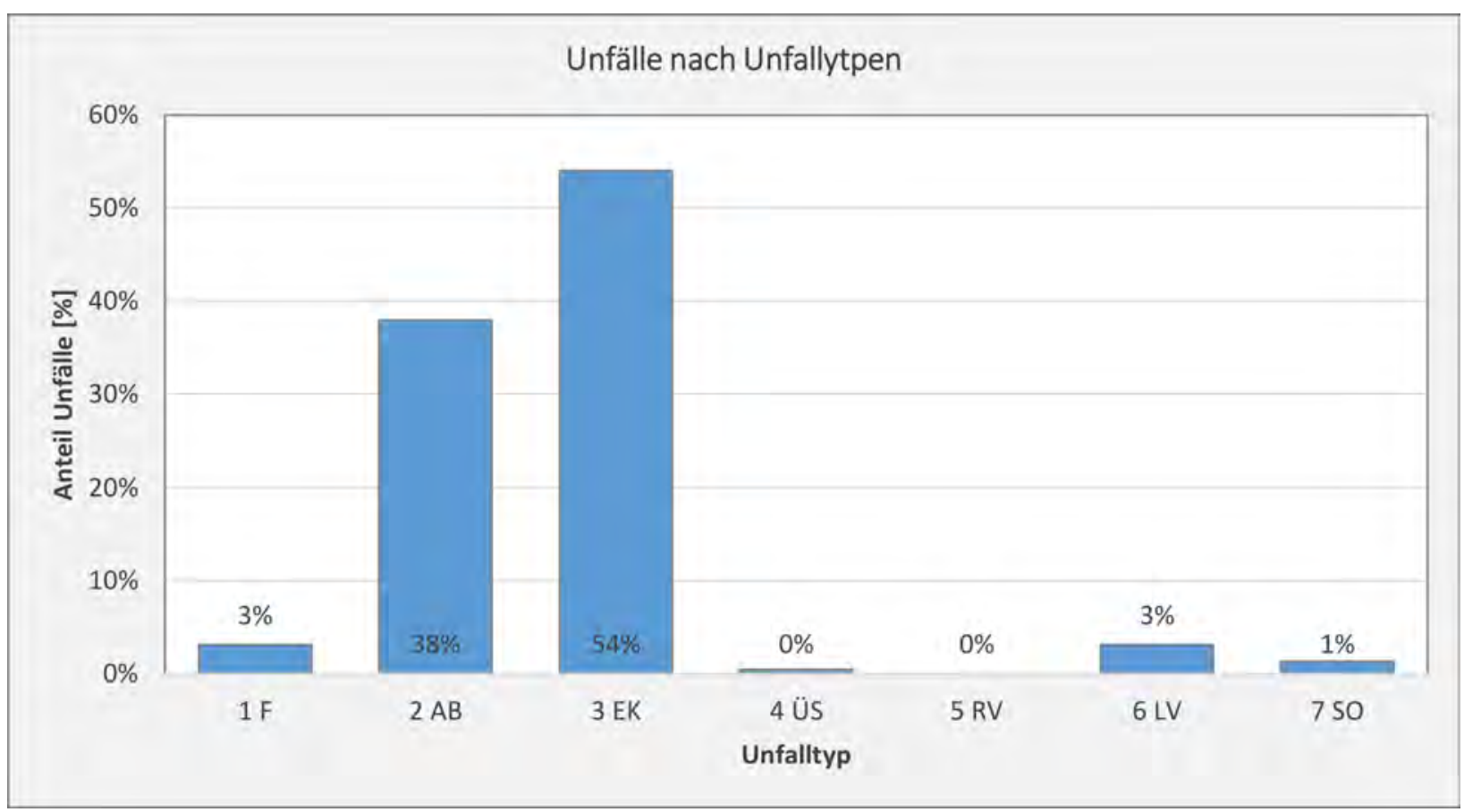

Abbildung 8: Unfälle nach Unfalltyp

Die Einteilung der Unfälle nach dem dreistelligen Unfalltypenkatalog liefert die folgenden Erkenntnisse:

- Bezogen auf den Unfalltyp 3 dominieren die beiden Unfalltypen 341 (Kraftfahrer biegt ein, Radfahrer kommt von links) (25,5 \% aller Unfälle) und 342 (Kraftfahrer biegt ein, Radfahrer kommt von rechts) $(22,3 \%$ aller Unfälle). Bei beiden Unfalltypen handelt es sich um Unfälle in den Kreisverkehrszufahrten zwischen in den Kreis einfahrenden Fahrzeugen und Radfahrern, die die Furten an den Querungsstellen befahren. Der Unfalltyp 341 umfasst die Unfälle mit von links kommenden Radfahrern und der Unfalltyp 342 die Unfälle mit von rechts kommenden Radfahrern.
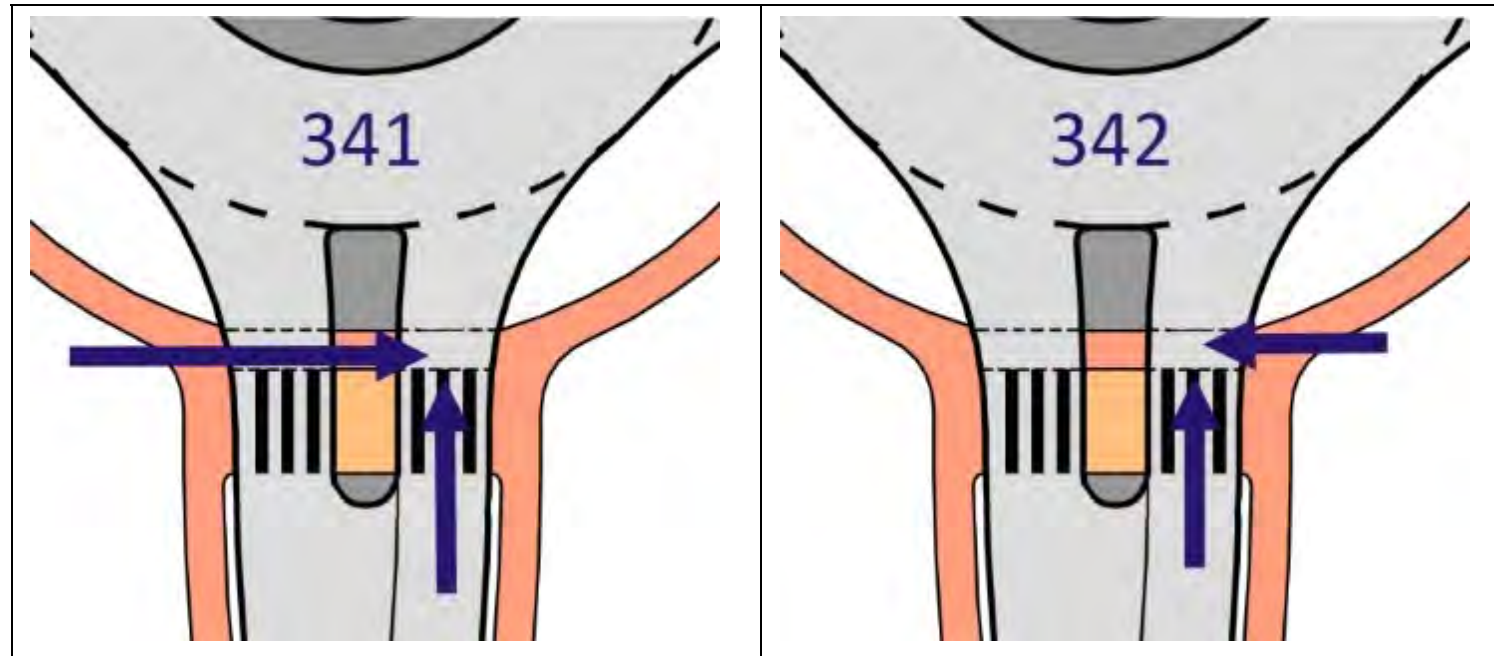
- Innerhalb der Abbiegeunfälle (Unfalltyp 2) dominiert der Unfalltyp 243 (Kraftfahrer biegt aus, Radfahrer kommt von rechts) mit 22,3\% aller Unfälle. Dieser Unfalltyp kennzeichnet Unfälle zwischen aus dem Kreis ausfahrenden Fahrzeugen und Radfahrern, die die Furt von rechts kommend überqueren. Zweit häufigster Unfalltyp ist der Unfalltyp 244 (Kraftfahrer biegt aus, Radfahrer kommt von links) mit 14,7\% aller Unfälle, zwischen aus dem Kreis ausfahrenden Fahrzeugen und Radfahrern, die die Furt von links kommend überqueren.

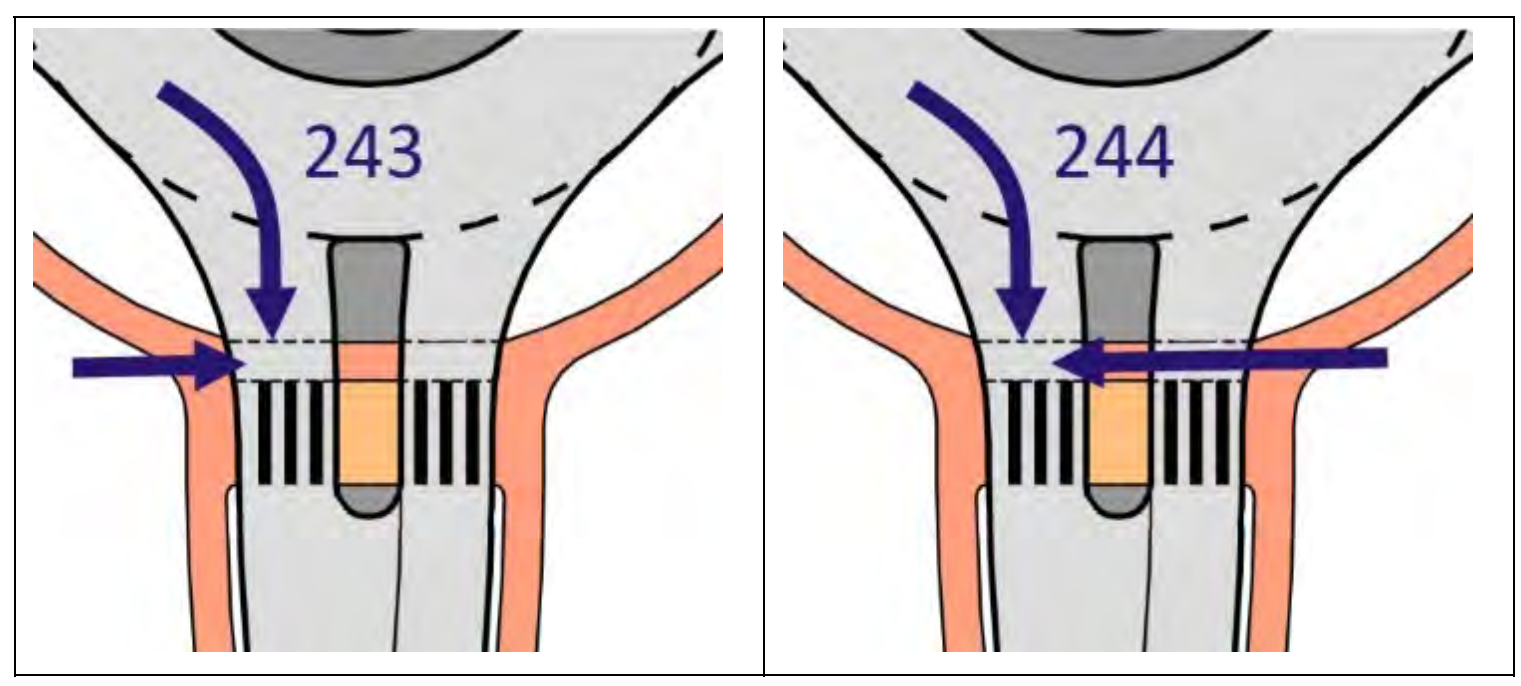

- Es besteht ein systematischer Unterschied zwischen Kreisverkehren mit Einrichtungsverkehr und mit Zweirichtungsverkehr. Bei Kreisverkehren mit Einrichtungsverkehr tritt der Unfalltyp 243 (Kraftfahrer biegt aus, Radfahrer kommt von rechts) wesentlich häufiger auf als an Kreisverkehren mit Zweirichtungsverkehr. Dem gegenüber treten an Kreisverkehren mit Zweirichtungsverkehr Unfälle des Typs 244 (Kraftfahrer biegt aus, Radfahrer kommt von links) etwa doppelt so häufig auf, wie an Kreisverkehren mit Einrichtungsverkehr. Bei Unfällen der Typen 341 (Kraftfahrer biegt ein, Radfahrer kommt von links) und 342 (Kraftfahrer fährt in den Kreis ein, Radfahrer kommt von rechts) sind keine Unterschiede erkennbar. 
Die folgende Abbildung zeigt die Anteile der einzelnen Unfalltypen bezogen auf alle Unfälle der Typen $243,244,341$ und 342.

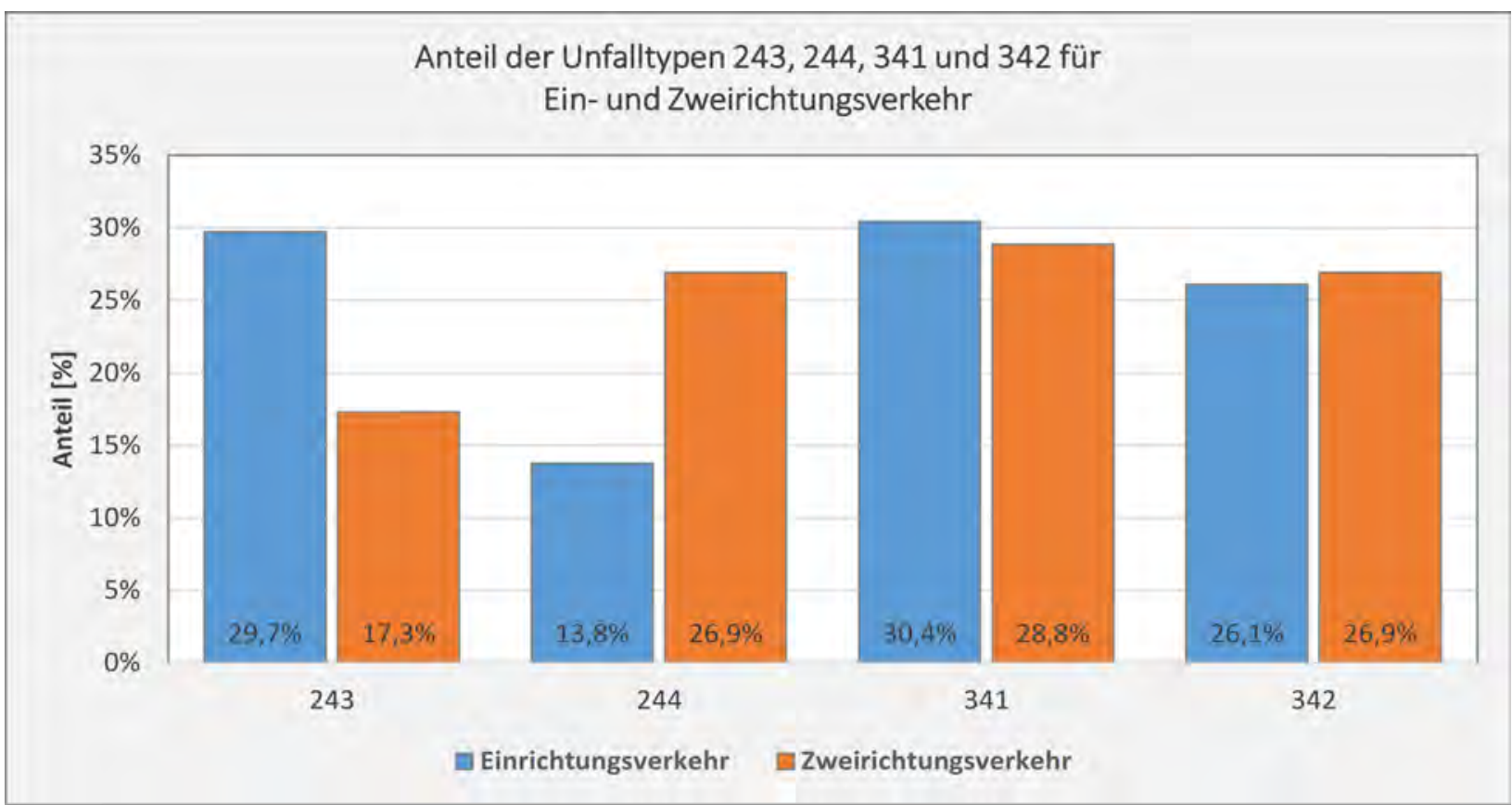

Abbildung 9: Differenzierung der Unfalltypen mit Radfahrerbeteiligung

Die folgende Abbildung zeigt die jeweils häufigsten Unfalltypen bezogen auf alle Unfälle mit Radfahrerbeteiligung.

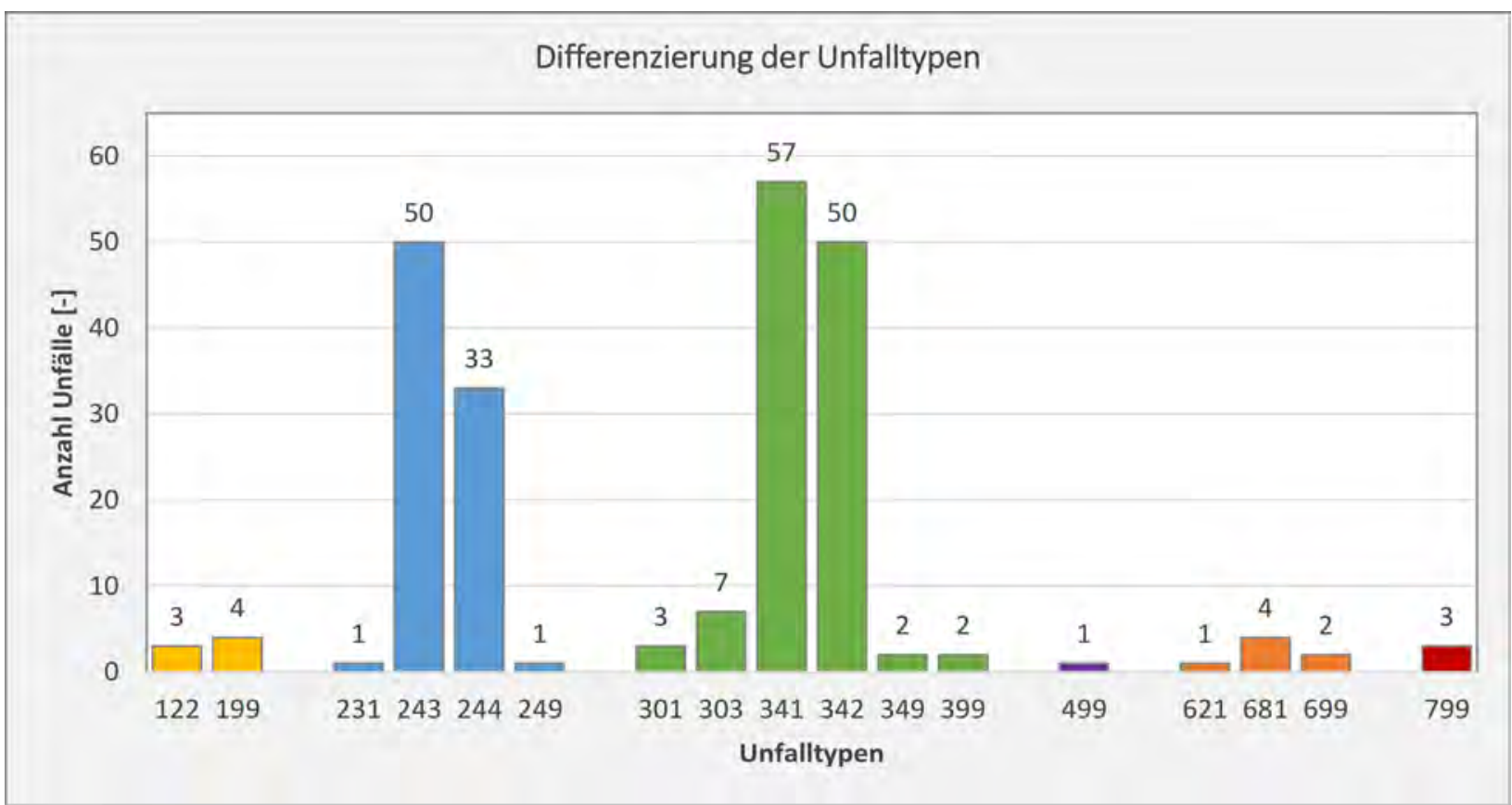

Abbildung 10: Differenzierung der Unfalltypen mit Radfahrerbeteiligung 


\subsection{Umfeldbedingungen}

Die Auswertung der Umfeldbedingungen wurde nur für die Unfälle der Typen 243, 244, 341 und 342 durchgeführt. Diese machen insgesamt 85 \% der Unfälle mit Radfahrerbeteiligung aus.

\section{Straßenzustand}

Etwa $22 \%$ der Unfälle ereigneten sich bei nasser oder feuchter Fahrbahn, $1 \%$ bei Glatteis und $71 \%$ bei trockener Fahrbahn. Bei 7 \% der Unfälle wurde der Straßenzustand nicht vermerkt.

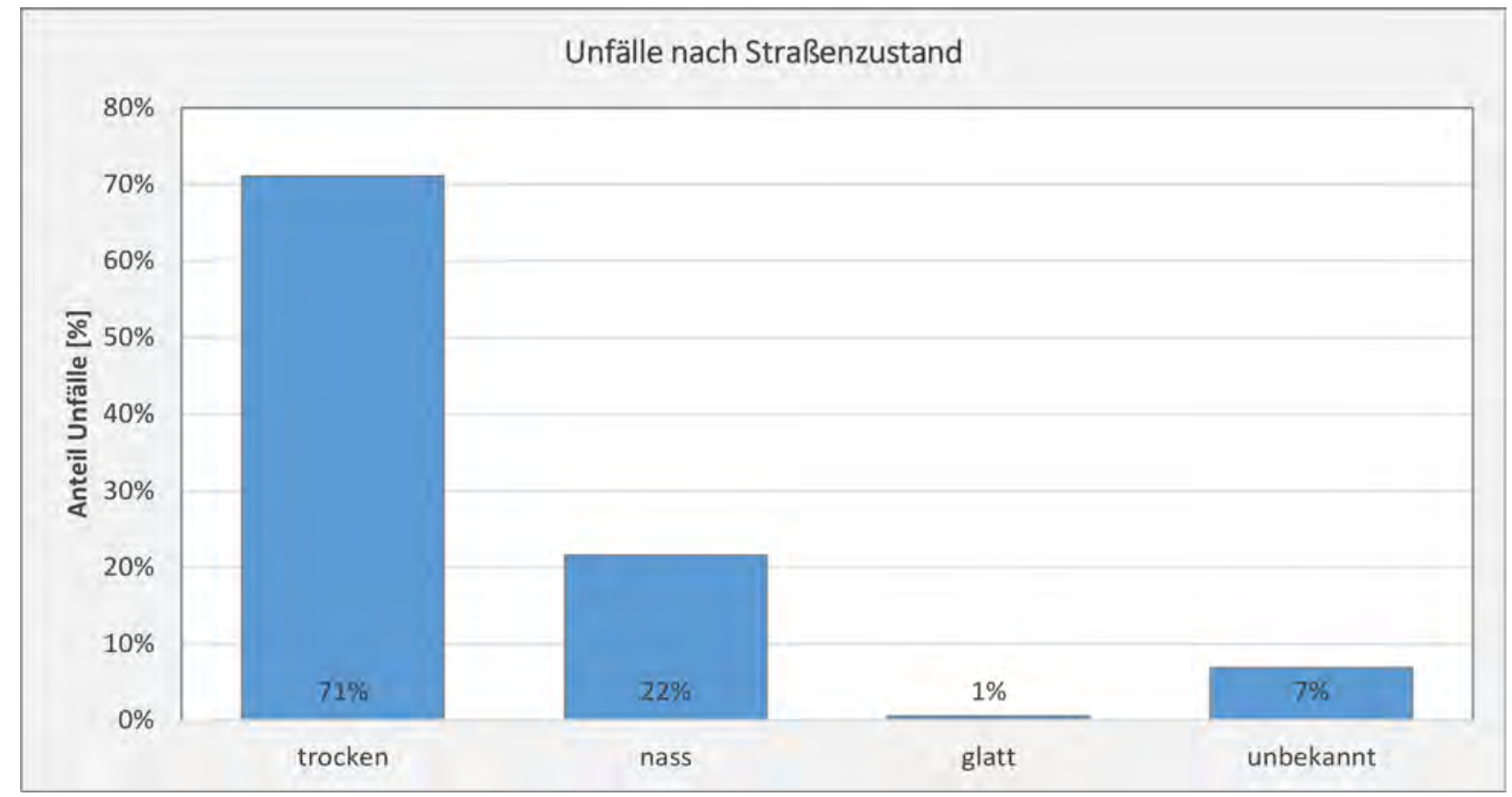

Abbildung 11: Unfälle nach Straßenzustand 


\section{Lichtverhältnisse}

Etwa $4 \%$ der Unfälle ereigneten sich bei Dämmerung, $10 \%$ bei Dunkelheit und $85 \%$ bei Tageslicht.

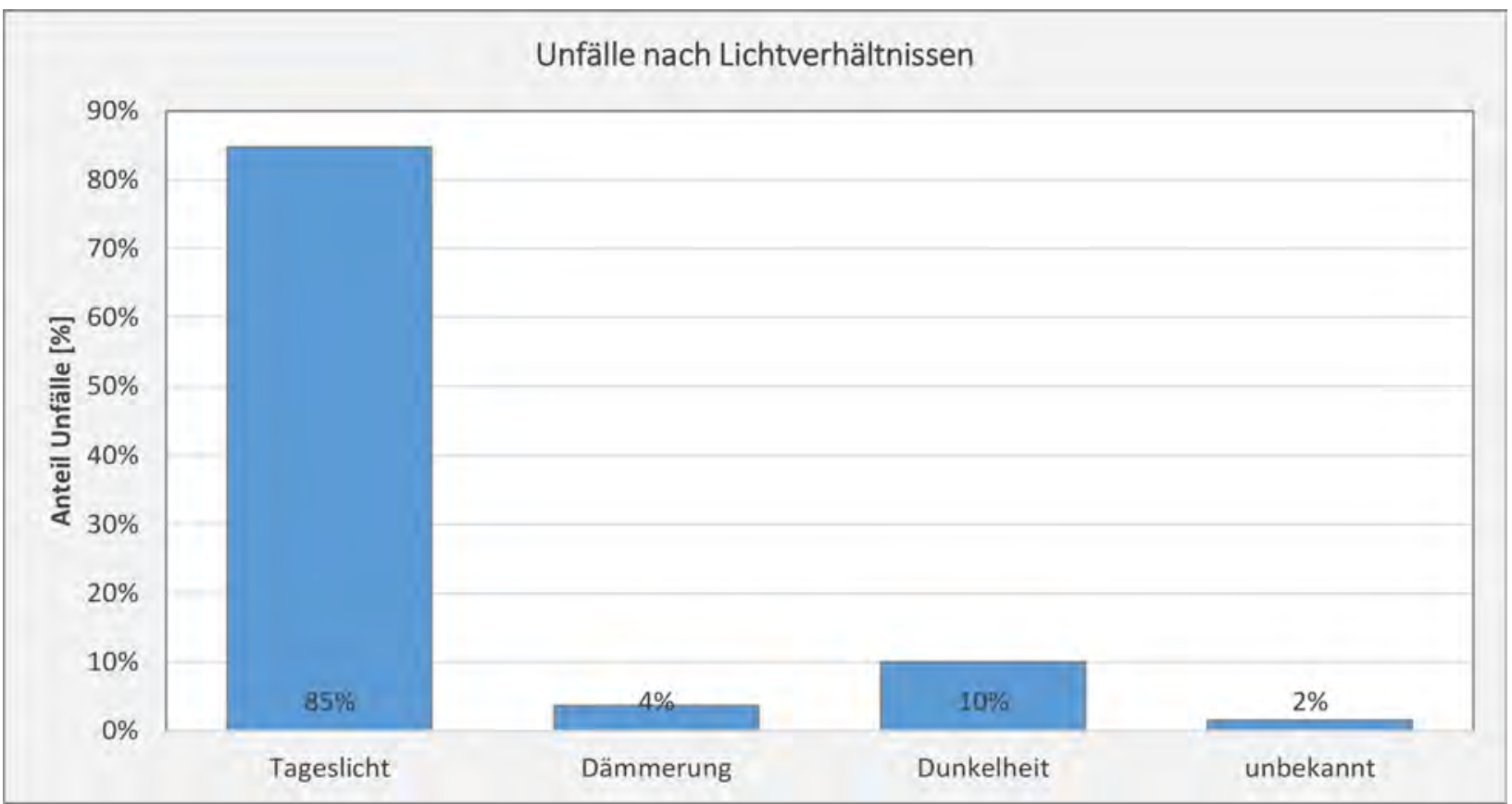

Abbildung 12: Unfälle nach Lichtverhältnissen 


\section{Bewertung der Verkehrssicherheit}

\subsection{Beschreibung der Unfallkenngrößen}

Die Beurteilung der Verkehrssicherheit erfolgt auf der Grundlage der folgenden Unfallkenngrößen:

- Unfalldichte (UD)

Die Unfalldichte beschreibt die durchschnittliche Anzahl der Unfälle im betrachteten Untersuchungszeitraum.

$$
\begin{array}{lll}
\text { UD }=\frac{U}{T}[U n f . / a] & \\
\text { mit: } \quad \text { UD } & = & \text { Unfalldichte } \\
\mathrm{U} & = & \begin{array}{l}
\text { Anzahl der Unfälle innerhalb des T Jahre umfassenden } \\
\text { Untersuchungszeitraums }
\end{array} \\
\mathrm{T} & = & \begin{array}{l}
\text { Länge des Untersuchungszeitraums in Jahren }
\end{array}
\end{array}
$$

- Unfallrate (UR)

Bei der Unfallrate wird die Anzahl der Unfälle auf das Verkehrsaufkommen bezogen (Unfälle pro $10^{6} \mathrm{Kfz}$ ). Sie errechnet sich nach der folgenden Gleichung:

$$
\begin{aligned}
& U R=\frac{U \cdot 10^{6}}{D T V \cdot 365 \cdot T}\left[U n f . / 10^{6} \mathrm{Kfz}\right] \\
& \text { mit: UR = Unfallrate } \\
& \mathrm{U} \quad=\quad \text { Anzahl der Unfälle innerhalb des } \mathrm{T} \text { Jahre umfassenden } \\
& \text { Untersuchungszeitraums } \\
& \text { DTV = } \quad \text { Summe des zuführenden Kfz-Verkehrs pro } 24 \mathrm{~h} \\
& \mathrm{~T} \quad=\quad \text { Länge des Untersuchungszeitraums in Jahren }
\end{aligned}
$$

- Unfallrate Radverkehr $\left(\mathrm{UR}_{\mathrm{R}}\right)$

Die eigentliche Unfallrate berücksichtigt ausschließlich die Kfz-Verkehrsbelastungen am Knotenpunkt. Es besteht die Vermutung, dass die Radverkehrsstärke an einem Kreisverkehr auch das Unfallgeschehen mit Radfahrerbeteiligung beeinflusst. Es wurde daher eine Unfallrate in die Untersuchungen einbezogen, die ausschließlich die Unfälle mit Radfahrerbeteiligung berücksichtigt und das Unfallgeschehen ausschließlich auf das Radverkehrsaufkommen bezieht. Um in etwa die gleiche Größenordnung zu erhalten, wie bei der klassischen Unfallrate wird die Unfallrate auf $10^{5}$ Radfahrer bezogen. Sie errechnet sich nach der folgenden Gleichung: 


$$
\begin{aligned}
& U R_{R}=\frac{U_{R} \cdot 10^{5}}{D T V_{R} \cdot 365 \cdot T}\left[\text { Unf . } / 10^{5} \mathrm{Rad}\right] \\
& \text { mit: } \quad \mathrm{UR}_{\mathrm{R}}=\text { Unfallrate Radverkehr } \\
& U_{\mathrm{R}}=\text { Anzahl der Unfälle mit Radfahrerbeteiligung innerhalb des } \\
& \text { T Jahre umfassenden Untersuchungszeitraums } \\
& \text { DTV }_{R}=\quad \text { Summe des Radverkehrs pro } 24 \mathrm{~h} \\
& \mathrm{~T}=\text { Länge des Untersuchungszeitraums in Jahren }
\end{aligned}
$$

- Unfallrate kombiniert Multiplikation $\left(\mathrm{UR}_{\mathrm{kM}}\right)$

Um das Kfz-Verkehrsaufkommen und das Radverkehrsaufkommen gleichermaßen zu berücksichtigen, wird zusätzlich eine kombinierte Berücksichtigung beider Verkehrsbelastungen vorgenommen. In Anlehnung an [7] erfolgt eine Multiplikation der Verkehrsbelastungen. Um die Werte in einer vergleichbarer Größenordnung zu halten wird die Zehnerpotenz im Zähler auf $10^{9}$ hochgesetzt.

Die kombinierte Unfallrate errechnet sich nach der folgenden Gleichung:

$$
\begin{aligned}
& U R_{k M}=\frac{U \cdot 10^{9}}{D T V_{K f z} \cdot D T V_{R} \cdot 365 \cdot T}\left[U n f . / 10^{9} \mathrm{Kfz} \cdot \mathrm{Rad}\right] \\
& \text { mit: } \quad \mathrm{UR}_{\mathrm{kM}}=\text { kombinierte Unfallrate } \\
& \mathrm{U} \quad=\quad \text { Anzahl der Unfälle innerhalb des T Jahre umfassenden } \\
& \text { Untersuchungszeitraums } \\
& \text { DTV }_{\mathrm{Kfz}}=\quad \text { Summe des zuführenden } \mathrm{Kfz} \text {-Verkehrs pro } 24 \mathrm{~h} \\
& \text { DTV }_{\mathrm{R}}=\quad \text { Summe des Radverkehrs pro } 24 \mathrm{~h} \\
& \mathrm{~T}=\quad \text { Länge des Untersuchungszeitraums in Jahren }
\end{aligned}
$$

- Unfallrate kombiniert Addition $\left(\mathrm{UR}_{\mathrm{kA}}\right)$

Zusätzlich wurde eine kombinierte Unfallrate mit Addition der Kfz- und Radverkehrsbelastungen nach der folgenden Gleichung errechnet.

$$
U R_{k A}=\frac{U \cdot 10^{6}}{D T V_{K f z}+D T V_{R} \cdot 365 \cdot T}\left[U n f . / 10^{6}(K f z+R a d)\right]
$$


mit: $\quad \mathrm{UR}_{\mathrm{kA}}=\quad$ kombinierte Unfallrate

$\mathrm{U} \quad=\quad$ Anzahl der Unfälle innerhalb des $\mathrm{T}$ Jahre umfassenden Untersuchungszeitraums

$\mathrm{DTV}_{\mathrm{Kfz}}=\quad$ Summe des zuführenden $\mathrm{Kfz}$-Verkehrs pro $24 \mathrm{~h}$

DTV $_{\mathrm{R}}=\quad$ Summe des Radverkehrs pro $24 \mathrm{~h}$

$\mathrm{T} \quad=\quad$ Länge des Untersuchungszeitraums in Jahren

- Unfallrate kombiniert Wurzelfunktion $\left(\mathrm{UR}_{\mathrm{kW}}\right)$

Zusätzlich wurde eine kombinierte Unfallrate mit Multiplikation der Kfz- und Radverkehrsbelastungen mit anschließender Wurzelbildung nach der folgenden Gleichung errechnet.

$$
\begin{aligned}
& U R_{\mathrm{kW}}=\frac{U \cdot 10^{6}}{\sqrt{D T V_{K f \mathrm{z}} \cdot \mathrm{DTV} \mathrm{Rad}_{\mathrm{d}}} \cdot 365 \cdot T}\left[U n f . / 10^{6} \sqrt{\mathrm{Kfz} \cdot \mathrm{Rad}}\right] \\
& \text { mit: } \quad \mathrm{UR}_{\mathrm{kW}}=\quad \text { kombinierte Unfallrate } \\
& \mathrm{U} \quad=\quad \text { Anzahl der Unfälle innerhalb des } \mathrm{T} \text { Jahre umfassenden } \\
& \text { Untersuchungszeitraums } \\
& \mathrm{DTV}_{\mathrm{Kfz}}=\quad \text { Summe des zuführenden } \mathrm{Kfz} \text {-Verkehrs pro } 24 \mathrm{~h} \\
& \text { DTV }_{\text {Rad }}=\quad \text { Summe des Radverkehrs pro } 24 \mathrm{~h} \\
& \mathrm{~T} \quad=\quad \text { Länge des Untersuchungszeitraums in Jahren }
\end{aligned}
$$

- Unfallkostendichte

Die Unfallkostendichte beschreibt die durchschnittlich jährlichen volkswirtschaftlichen Kosten die im Untersuchungszeitraum am Knotenpunkt entstehen. Sie errechnet sich nach folgender Gleichung:

$$
\begin{array}{lll}
\text { UKD }=\frac{U K}{T}[€ / a] & \\
\text { mit: } \quad \text { UKD } & = & \text { Unfallkostendichte } \\
\text { UK } & = & \begin{array}{l}
\text { Unfallkosten innerhalb des T Jahre umfassenden } \\
\text { Untersuchungszeitraums }
\end{array} \\
\mathrm{T} & = & \text { Länge des Untersuchungszeitraums in Jahren }
\end{array}
$$


- Unfallkostenrate

Bei der Unfallkostenrate werden die volkswirtschaftlichen Kosten durch Straßenverkehrsunfälle auf das Verkehrsaufkommen bezogen ( $€$ pro $10^{3} \mathrm{Kfz}$ ). Sie errechnet sich nach der folgenden Gleichung:

$$
\begin{array}{ll}
\text { UKR }=\frac{10^{3} \cdot U K}{D T V \cdot 365 \cdot T}\left[€ / 10^{3} \mathrm{Kfz}\right] \\
\text { mit: } \begin{array}{ll}
\text { UKR }= & \text { Unfallkostenrate } \\
\text { UK }= & \begin{array}{l}
\text { Unfallkosten innerhalb des T Jahre umfassenden } \\
\text { Untersuchungszeitraums }
\end{array} \\
\text { DTV }= & \text { Summe des zuführenden Kfz-Verkehrs pro } 24 \mathrm{~h} \\
\mathrm{~T} \quad= & \text { Länge des Untersuchungszeitraums in Jahren }
\end{array}
\end{array}
$$

- Unfallkostenrate Radverkehr $\left(\mathrm{UKR}_{\mathrm{R}}\right)$

Die eigentliche Unfallkostenrate berücksichtigt ausschließlich die Kfz-Verkehrsbelastungen am Knotenpunkt. Es wird angenommen, dass die Radverkehrsstärke an einem Kreisverkehr auch das Unfallgeschehen mit Radfahrerbeteiligung beeinflusst. Es wurde daher eine Unfallkostenrate in die Untersuchungen einbezogen, die ausschließlich die Unfälle mit Radfahrerbeteiligung berücksichtigt und das Unfallgeschehen ausschließlich auf das Radverkehrsaufkommen bezieht. Um in etwa die gleiche Größenordnung zu erhalten, wie bei der klassischen Unfallkostenrate wird die Unfallrate auf $10^{2}$ Radfahrer bezogen. Sie errechnet sich nach der folgenden Gleichung:

$$
\begin{aligned}
& U K R_{R}=\frac{10^{2} \cdot U K_{R}}{D T V_{R} \cdot 365 \cdot T}\left[€ / 10^{2} \mathrm{Rad}\right] \\
& \text { mit: } \quad \mathrm{UKR}_{\mathrm{R}}=\quad \text { Unfallkostenrate Radverkehr } \\
& \mathrm{UK}_{\mathrm{R}}=\quad \text { Unfallkosten der Unfälle mit Radfahrerbeteiligung innerhalb des T Jahre } \\
& \text { umfassenden Untersuchungszeitraums } \\
& \text { DTV }_{\mathrm{R}}=\quad \text { Summe des Radverkehrs pro } 24 \mathrm{~h} \\
& \mathrm{~T}=\quad \text { Länge des Untersuchungszeitraums in Jahren }
\end{aligned}
$$

- Unfallkostenrate kombiniert Multiplikation $\left(\mathrm{UKR}_{\mathrm{kM}}\right)$

Um das Kfz-Verkehrsaufkommen und das Radverkehrsaufkommen gleichermaßen zu berücksichtigen, wird zusätzlich eine kombinierte Berücksichtigung beider Verkehrsbelastungen vorgenommen. In Anlehnung an [7] erfolgt eine Multiplikation der Verkehrsbelastungen. Um die Werte in einer vergleichbarer Größenordnung zu halten wird die Zehnerpotenz im Zähler auf $10^{6}$ hochgesetzt. 
Die kombinierte Unfallkostenrate errechnet sich nach der folgenden Gleichung:

$$
\begin{aligned}
& U K R_{k M}=\frac{10^{6} \cdot U K}{D T V_{K f z} \cdot D T V_{R} \cdot 365 \cdot T}\left[€ / 10^{6} \mathrm{Kfz} \cdot \mathrm{Rad}\right] \\
& \text { mit: } \quad \mathrm{UKR}_{\mathrm{kM}}=\quad \text { kombinierte Unfallkostenrate } \\
& \mathrm{UK}_{\mathrm{k}}=\text { Unfallkosten innerhalb des T Jahre umfassenden } \\
& \text { Untersuchungszeitraums } \\
& \mathrm{DTV}_{\mathrm{Kfz}}=\quad \text { Summe des zuführenden } \mathrm{Kfz} \text {-Verkehrs pro } 24 \mathrm{~h} \\
& \text { DTV }_{\mathrm{R}}=\quad \text { Summe des Radverkehrs pro } 24 \mathrm{~h} \\
& \mathrm{~T}=\quad \text { Länge des Untersuchungszeitraums in Jahren }
\end{aligned}
$$

- Unfallkostenrate kombiniert Addition (UKR $\left.\mathrm{KA}_{\mathrm{K}}\right)$

Eine Alternative zur Berücksichtigung des Kfz- und Radverkehrsaufkommens stellt die Addition der KfZ- und Radverkehrsbelastungen dar.

Die daraus errechnete kombinierte Unfallkostenrate errechnet sich nach der folgenden Gleichung:

$$
\begin{aligned}
& U K R_{k A}=\frac{10^{3} \cdot U K}{D T V_{K f z}+D T V_{R} \cdot 365 \cdot T}\left[€ / 10^{3}(K f z+R a d)\right] \\
& \text { mit: } \quad \mathrm{UKR}_{\mathrm{kA}}=\quad \text { kombinierte Unfallkostenrate } \\
& \mathrm{UK}_{\mathrm{k}}=\quad \text { Unfallkosten innerhalb des } \mathrm{T} \text { Jahre umfassenden } \\
& \text { Untersuchungszeitraums } \\
& \mathrm{DTV}_{\mathrm{Kfz}}=\quad \text { Summe des zuführenden } \mathrm{Kfz} \text {-Verkehrs pro } 24 \mathrm{~h} \\
& \mathrm{DTV}_{\mathrm{R}}=\quad \text { Summe des Radverkehrs pro } 24 \mathrm{~h} \\
& \mathrm{~T}=\quad \text { Länge des Untersuchungszeitraums in Jahren }
\end{aligned}
$$

- Unfallkostenrate kombiniert Wurzelbildung $\left(\mathrm{UKR}_{\mathrm{kW}}\right)$

Zusätzlich wurde eine kombinierte Unfallkostenrate mit Multiplikation der $\mathrm{Kfz}$ - und Radverkehrsbelastungen mit anschließender Wurzelbildung nach der folgenden Gleichung errechnet. 


$$
\begin{aligned}
& U K R_{k W}=\frac{10^{3} \cdot U K}{\sqrt{D T V_{K f z} \cdot D T V_{R a d}} \cdot 365 \cdot T}\left[€ / 10^{3} \sqrt{K f z \cdot R a d}\right] \\
& \text { mit: } \quad \mathrm{UKR}_{\mathrm{kW}}=\quad \text { kombinierte Unfallkostenrate } \\
& \mathrm{UK}_{\mathrm{k}}=\quad \text { Unfallkosten innerhalb des T Jahre umfassenden } \\
& \text { Untersuchungszeitraums } \\
& \text { DTV }_{\mathrm{Kfz}}=\quad \text { Summe des zuführenden } \mathrm{Kfz} \text {-Verkehrs pro } 24 \mathrm{~h} \\
& \text { DTV }_{\text {Rad }}=\quad \text { Summe des Radverkehrs pro } 24 \mathrm{~h} \\
& \mathrm{~T}=\text { Länge des Untersuchungszeitraums in Jahren }
\end{aligned}
$$

\subsection{Unfallrate}

Die folgende Tabelle zeigt die Ergebnisse der Berechnungen für die folgenden, unter 6.1 beschriebenen Unfallraten:

- Unfallrate UR

- Unfallrate Radverkehr $\mathrm{UR}_{\mathrm{R}}$

- Unfallrate kombiniert Multiplikation $\mathrm{UR}_{\mathrm{kM}}$

- Unfallrate kombiniert Addition UR $\mathrm{R}_{\mathrm{kA}}$

- Unfallrate kombiniert Wurzelfunktion $\mathrm{UR}_{\mathrm{kW}}$

Neben dem Mittelwert sind zudem die Standardabweichung sowie der Variationskoeffizient angegeben.

\begin{tabular}{|l|c|c|c|}
\hline Unfallkenngröße & Mittelwert & Standardabweichung & Variationskoeffizient \\
\hline UR & $\mathbf{x}$ & $\mathbf{s}$ & $\mathbf{v}$ \\
\hline $\mathrm{UR}_{\mathrm{R}}$ & 0,09 & 0,19 & 2,12 \\
\hline $\mathrm{UR}_{\mathrm{kM}}$ & 0,29 & 0,59 & 1,92 \\
\hline $\mathrm{UR}_{\mathrm{kA}}$ & 0,32 & 0,61 & 1,85 \\
\hline $\mathrm{UR}_{\mathrm{kW}}$ & 0,08 & 0,15 & 1,92 \\
\hline
\end{tabular}

Tabelle 1: Vergleich der Unfallraten - Statistische Kennwerte

Die Gegenüberstellung zeigt, dass die drei kombinierten Unfallraten die insgesamt niedrigsten Variationskoeffizienten und damit die geringsten Streuungen aufweisen. 


\subsection{Unfallkostenrate}

Die folgende Tabelle zeigt die Ergebnisse der Berechnungen für die folgenden, unter 6.1 beschriebenen Unfallraten:

- Unfallkostenrate UR

- Unfallkostenrate Radverkehr $\mathrm{UR}_{\mathrm{R}}$

- Unfallkostenrate kombiniert Multiplikation $U_{\mathrm{kM}}$

- Unfallkostenrate kombiniert Addition $\mathrm{UR}_{\mathrm{kA}}$

- Unfallkostenrate kombiniert Wurzelfunktion $U_{\mathrm{kW}}$

Neben dem Mittelwert sind zudem die Standardabweichung sowie der Variationskoeffizient angegeben.

\begin{tabular}{|l|c|c|c|}
\hline Unfallkenngröße & Mittelwert & Standardabweichung & Variationskoeffizient \\
\hline UKR & $\mathbf{x}$ & 6,07 & $\mathbf{\text { s }}$ \\
\hline $\mathrm{UKR}_{\mathrm{R}}$ & 1,83 & 14,94 & 2,80 \\
\hline $\mathrm{UKR}_{\mathrm{kM}}$ & 5,34 & 29,83 & 4,28 \\
\hline $\mathrm{UKR}_{\mathrm{kA}}$ & 6,97 & 5,45 & 3,29 \\
\hline $\mathrm{UKR}_{\mathrm{kW}}$ & 1,65 & 22,42 & 2,75 \\
\hline
\end{tabular}

Tabelle 2: Vergleich der Unfallraten - Statistische Kennwerte

Die Gegenüberstellung zeigt, dass die Unfallkostenrate kombiniert Wurzelfunktion $\mathrm{UKR}_{\mathrm{kW}}$ sowie die Unfallkostenrate Radverkehr $\mathrm{UKR}_{\mathrm{R}}$ die deutlich niedrigsten Variationskoeffizienten und damit die geringsten Streuungen aufweisen.

\section{$6.4 \quad$ Fazit}

Bei gemeinsamer Betrachtung der Unfallrate und der Unfallkostenrate zeigt sich, dass die Berechnungsmethode mit Multiplikation der Verkehrsstärken im Kfz-Verkehr mit den Radverkehrsverstärken und anschließender Wurzelbildung die insgesamt geringsten Variationskoeffizienten aufweist. Die weiteren Auswertungen werden daher im Wesentlichen mit diesen Unfallkenngrößen durchgeführt. 


\section{$7 \quad$ Verkehrsstärke und Unfallgeschehen}

\subsection{Kfz-Verkehrsbelastungen}

Der Zusammenhang zwischen den Kfz-Verkehrsbelastungen und den Unfallkenngrößen wurde mittels Regressionsrechnungen analysiert. Die folgende Tabelle zeigt die errechneten Bestimmtheitsmaße. Dabei zeigt sich, dass kein linearer Zusammenhang zwischen dem Kfz-Verkehrsaufkommen und den Unfallkenngrößen besteht.

\begin{tabular}{|l|l|c|}
\hline Unabhängige Variable & \multicolumn{1}{|c|}{ Abhängige Variable } & Bestimmtheitsmaß \\
\hline DTV (Kfz / 24h ) & UR $_{\mathrm{kW}}$ & 0,00 \\
\hline DTV (Kfz / 24h ) & UKR $_{\mathrm{kW}}$ & 0,01 \\
\hline DTV (Kfz / 24h ) & UD & 0,01 \\
\hline DTV (Kfz / 24h $)$ & UKD & 0,00 \\
\hline
\end{tabular}

Tabelle 3: Bestimmtheitsmaße

\subsection{Radverkehrsbelastungen}

Es wurde geprüft, inwieweit ein Zusammenhang zwischen den Radverkehrsbelastungen und den Unfallkenngrößen der Unfälle mit Radfahrerbeteiligung besteht. Die folgende Tabelle zeigt die errechneten Bestimmtheitsmaße.

\begin{tabular}{|l|l|c|}
\hline Unabhängige Variable & \multicolumn{1}{|c|}{ Abhängige Variable } & Bestimmtheitsmaß \\
\hline DTV $(\operatorname{Rad} / 24 \mathrm{~h})$ & $\mathrm{UR}_{\mathrm{kW}}$ & 0,01 \\
\hline $\mathrm{DTV}(\operatorname{Rad} / 24 \mathrm{~h})$ & $\mathrm{UKR}_{\mathrm{kW}}$ & 0,01 \\
\hline $\mathrm{DTV}(\operatorname{Rad} / 24 \mathrm{~h})$ & $\mathrm{UD}$ & 0,18 \\
\hline $\mathrm{DTV}(\operatorname{Rad} / 24 \mathrm{~h})$ & UKD & 0,13 \\
\hline
\end{tabular}

Tabelle 4: Bestimmtheitsmaße

Lediglich zwischen der Unfalldichte UD und der Unfallkostendichte UKD besteht ein leichter linearer Zusammenhang mit den Radverkehrsbelastungen. Die folgenden Abbildungen zeigen den Verlauf der Regressionsgeraden. 


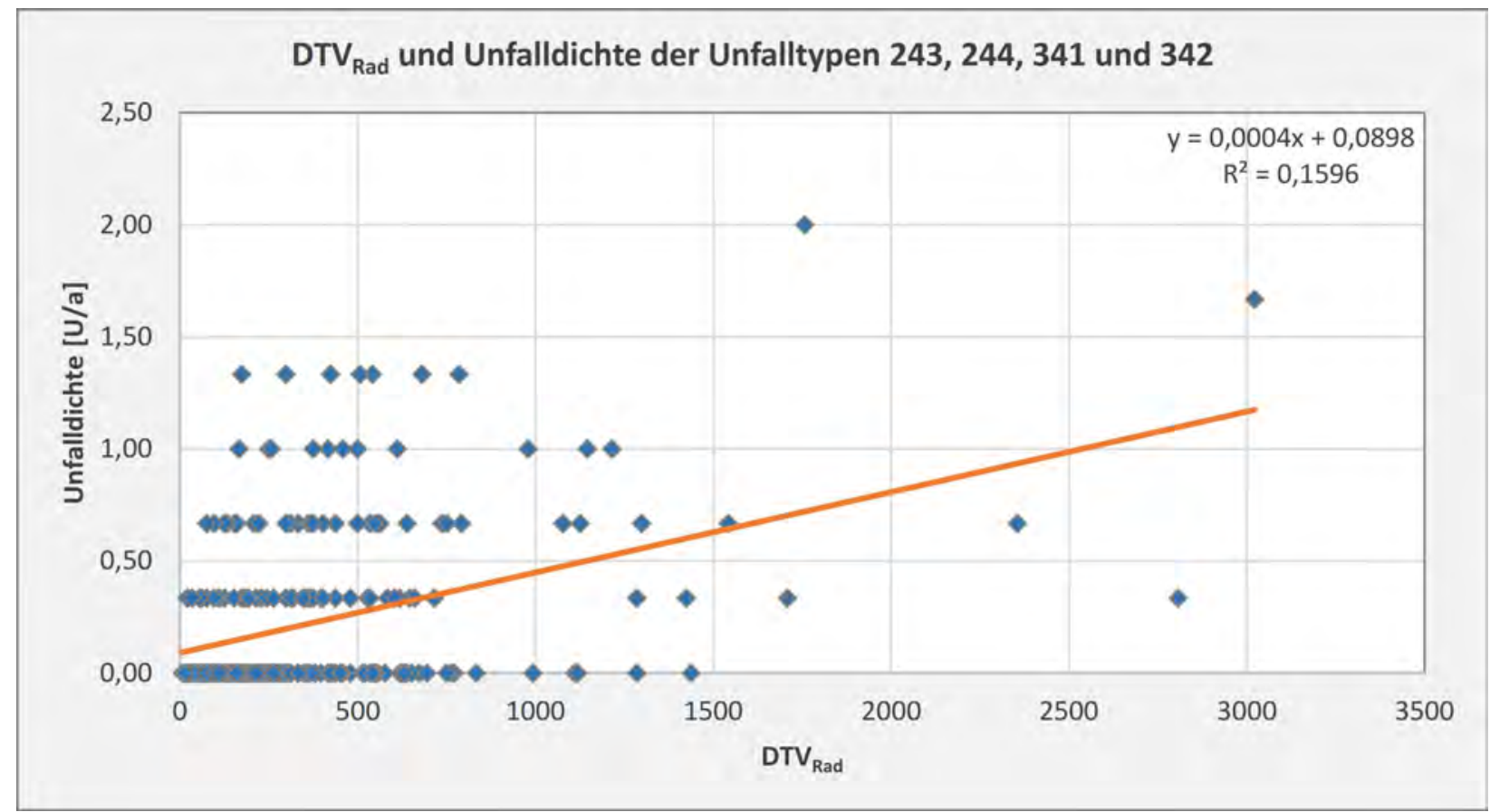

Abbildung 13: Zusammenhang zwischen Radverkehrsbelastungen und Unfalldichte (Unfälle mit Radfahrerbeteiligung)

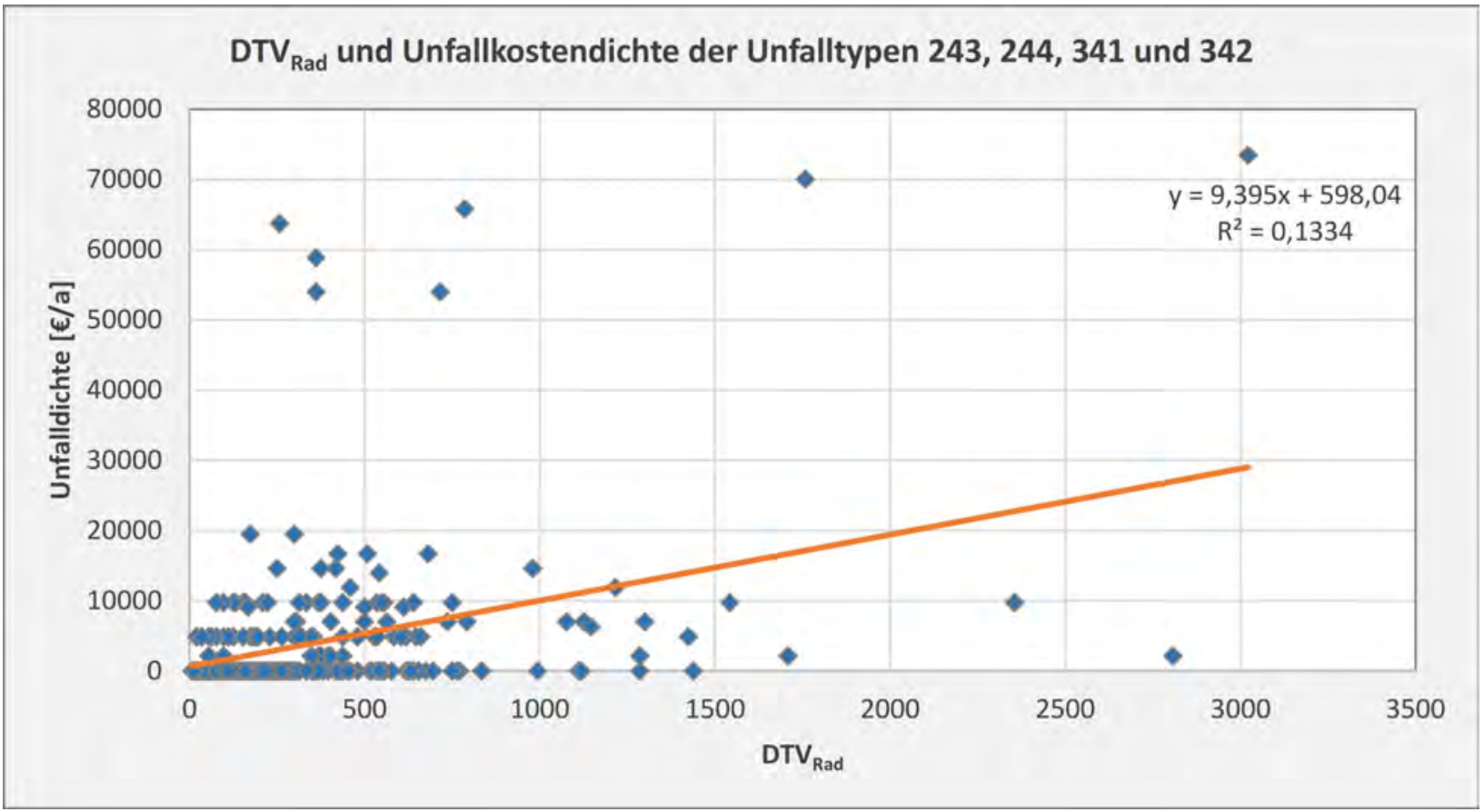

Abbildung 14: Zusammenhang zwischen Radverkehrsbelastungen und Unfallkostendichte (Unfälle mit Radfahrerbeteiligung) 


\subsection{Kombinierte Verkehrsbelastungen}

Es wurde geprüft, inwieweit ein Zusammenhang zwischen den kombinierten Verkehrsbelastungen und den Unfallkenngrößen der Unfälle mit Radfahrerbeteiligung besteht. Die folgende Tabelle zeigt die errechneten Bestimmtheitsmaße.

\begin{tabular}{|c|l|c|}
\hline Unabhängige Variable & \multicolumn{1}{|c|}{ Abhängige Variable } & Bestimmtheitsmaß \\
\hline $\mathrm{DTV}(\mathrm{Kfz} * \mathrm{Rad} / 24 \mathrm{~h})$ & Unfalldichte & 0,18 \\
\hline $\mathrm{DTV}(\mathrm{Kfz} * \mathrm{Rad} / 24 \mathrm{~h})$ & Unfallkostendichte & 0,15 \\
\hline $\mathrm{DTV}(\mathrm{Kfz}+\mathrm{Rad} / 24 \mathrm{~h})$ & Unfalldichte & 0,01 \\
\hline $\mathrm{DTV}(\mathrm{Kfz}+\mathrm{Rad} / 24 \mathrm{~h})$ & Unfallkostendichte & 0,01 \\
\hline $\mathrm{DTV}(\sqrt{K f z * R a d} / 24 h$ & Unfalldichte & 0,19 \\
\hline $\mathrm{DTV}(\sqrt{K f z * \operatorname{Rad} / 24 h}$ & Unfallkostendichte & 0,12 \\
\hline
\end{tabular}

Tabelle 5: Bestimmtheitsmaße

Es bestehen leichte lineare Zusammenhänge zwischen den mittels Multiplikation verknüpften Verkehrsbelastungen und der Unfalldichte bzw. der Unfallkostendichte sowie zwischen den mittels Multiplikation und anschließender Wurzelbildung verknüpften Verkehrsbelastungen und der Unfalldichte bzw. der Unfallkostendichte. Zwischen den mittels Addition verknüpften Verkehrsbelastungen und der Unfalldichte bzw. der Unfallkostendichte besteht hingegen kein Zusammenhang.

\subsection{Bewertung}

Hinsichtlich des Zusammenhangs zwischen den Verkehrsbelastungen und den Unfallkenngrößen kommt die Untersuchung zu den folgenden Ergebnissen:

- Es besteht kein linearer Zusammenhang zwischen den Kfz-Verkehrsbelastungen und den Unfallkenngrößen.

- Es besteht ein schwacher linearer Zusammenhang zwischen den Radverkehrsbelastungen und der Unfalldichte (Bestimmtheitsmaß 0,18) sowie der Unfallkostendichte (Bestimmtheitsmaß 0,13).

- Es besteht ein schwacher linearer Zusammen zwischen den mittels Multiplikation verknüpften Verkehrsbelastungen und der Unfalldichte (Bestimmtheitsmaß 0,18 ) sowie der Unfallkostendichte (Bestimmtheitsmaß 0,15).

- Es besteht ein schwacher linearer Zusammen zwischen den mittels Multiplikation und anschließender Wurzelbildung verknüpften Verkehrsbelastungen und der Unfalldichte (Bestimmtheitsmaß 0,19) sowie der Unfallkostendichte (Bestimmtheitsmaß 0,12).

- Es besteht kein Zusammenhang zwischen den mittels Addition verknüpften Verkehrsbelastungen und den Unfallkenngrößen.

- Die unter Ziffer 6 erläuterten Ergebnisse werden bestätigt. Die mittels Multiplikation und anschließender Wurzelbildung kombinierten Kfz- und Radverkehrsbelastungen können als geeignete Parameter in die Berechnung der Unfallrate und Unfallkostenrate einbezogen werden 


\section{Einzelmerkmale und Unfallgeschehen}

\subsection{Allgemeines}

Im Rahmen der Untersuchung wurde an jedem Kreisverkehrsarm bei einer Ortsbesichtigung ein Datenblatt mit den charakteristischen Merkmalen ausgefüllt. Die folgende Abbildung zeigt die erfassten Merkmale:

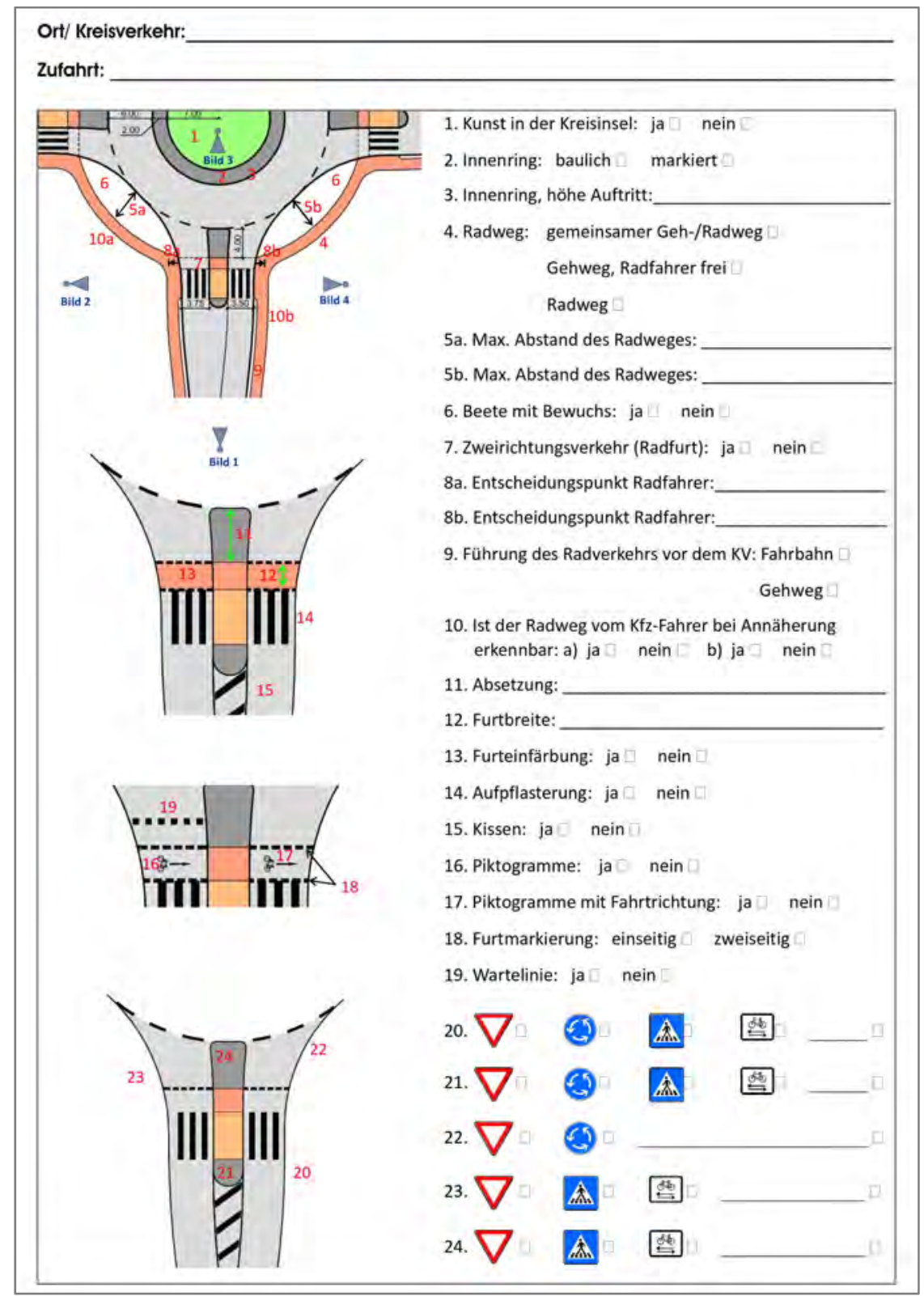

Abbildung 15: Datenblatt

Die Auswertung der Einzelmerkmale wurde nur für die Unfälle der Typen 243, 244, 341 und 342 durchgeführt. Diese machen insgesamt $85 \%$ der Unfälle mit Radfahrerbeteiligung aus. 


\subsection{Außendurchmesser}

Die Kreisverkehre wurden in die folgenden Außendurchmesserklassen eingeteilt:

- Unter 29,5 m:

- $29,5 \mathrm{~m}$ bis $37,9 \mathrm{~m}$ :

- 38 m und mehr:
39 Kreisverkehrsarme (13\%)

209 Kreisverkehrsarme (72 \%)

44 Kreisverkehrsarme (15\%)

Bei Betrachtung aller Unfalltypen zeigt sich, dass die Kreisverkehrsarme an Kreisverkehren mit Außendurchmessern unter 29,5 m höhere Unfallkenngrößen aufweisen. Die Unfallkostenrate liegt um etwa $25 \%$ höher als bei größeren Außendurchmessern. Die $95 \%$-Konfidenzintervalle überschneiden sich jedoch sehr stark, so dass eine Signifikanz der Unterschiede nicht nachgewiesen werden kann.

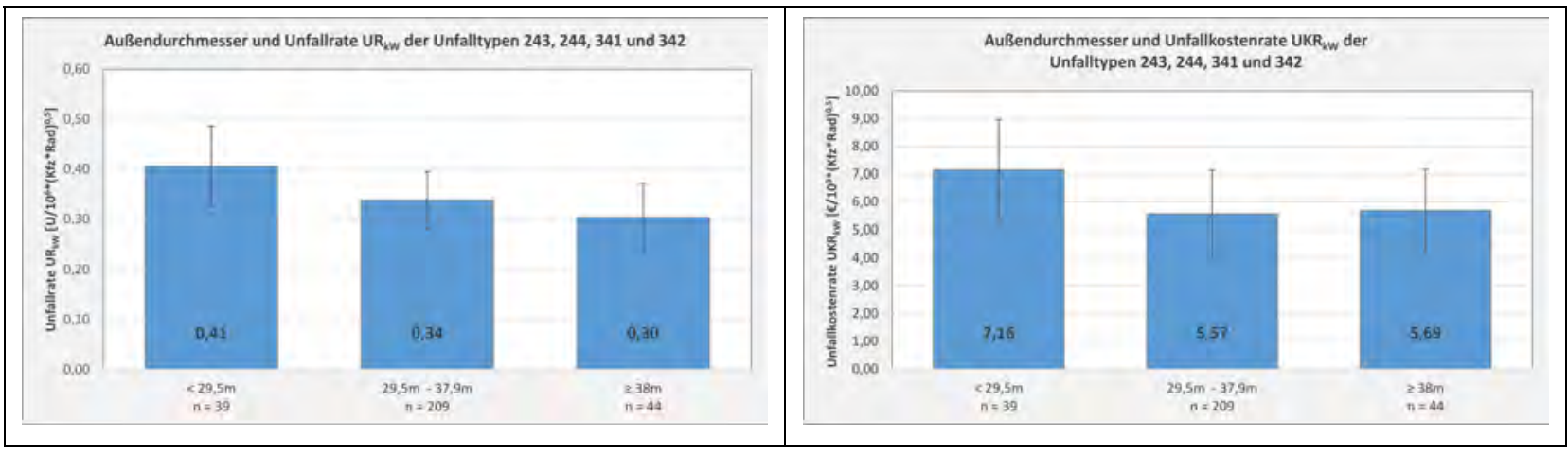

Abbildung 16: Außendurchmesser

Die Auswertung zeigt, dass der Außendurchmesser insbesondere die Unfälle in den Ausfahrten beeinflusst. Die Unfallkostenrate der Ausfahrten an Kreisverkehren mit Außendurchmessern unter 29,5 m liegt um etwa $40 \%$ höher als bei größeren Außendurchmessern. Die $95 \%$-Konfidenzintervalle überschneiden sich jedoch sehr stark, so dass eine Signifikanz der Unterschiede nicht nachgewiesen werden kann.
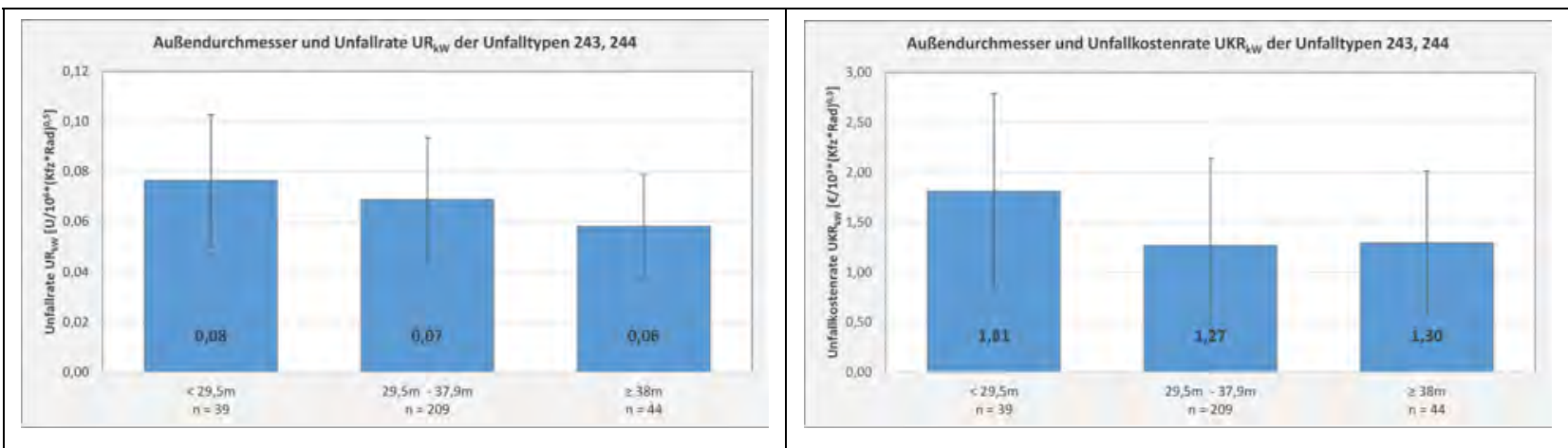

Abbildung 17: Außendurchmesser und Unfälle in den Ausfahrten

Bezogen auf die Unfälle in den Zufahrten sind die Unterschiede weniger deutlich. Die Unfallkostenrate der Zufahrten an Kreisverkehren mit Außendurchmesser unter 29,5 m liegt um etwa $15 \%$ höher als bei größeren Außendurchmessern bei starker Überschneidung der Konfidenzintervalle. 


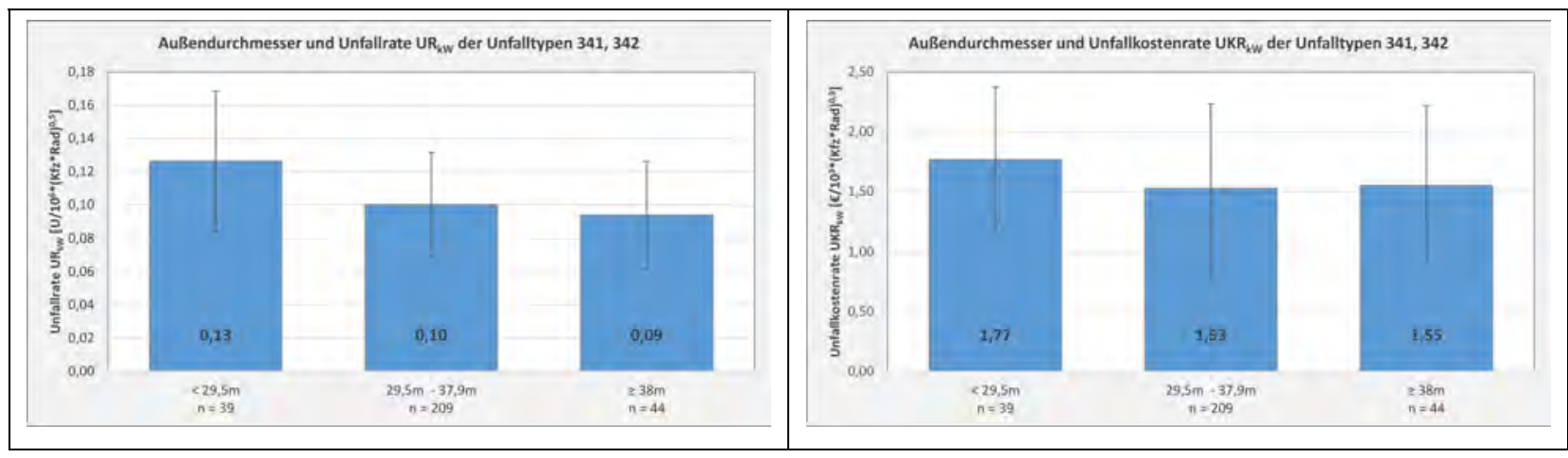

Abbildung 18: Außendurchmesser und Unfälle in den Zufahrten

\subsection{Durchmesser der Kreisinsel}

Gemäß der Untersuchung von Jensen [12] aus Dänemark hat der Durchmesser der Kreisinsel einen Einfluss auf die Verkehrssicherheit der Radfahrer. Die Kreisverkehre wurden in die folgenden Durchmesserklassen eingeteilt:

- Unter 12,7 m:

- $12,7 \mathrm{~m}$ bis $16,7 \mathrm{~m}$ :

- $16,8 \mathrm{~m}$ bis $19,7 \mathrm{~m}$ :

- $19,8 \mathrm{~m}$ bis $22,9 \mathrm{~m}$ :

- 23 m und mehr:
43 Kreisverkehrsarme (15\%)

91 Kreisverkehrsarme (31\%)

59 Kreisverkehrsarme (20 \%)

59 Kreisverkehrsarme (20\%)

40 Kreisverkehrsarme (14\%)

Bei Betrachtung aller Unfalltypen zeigt sich, dass die Kreisverkehrsarme an Kreisverkehren mit kleinen Kreiselinseldurchmessern unter 12,7 m höhere Unfallkostenraten aufweisen. Die $95 \%$ Konfidenzintervalle überschneiden sich jedoch, so dass eine Signifikanz der Unterschiede nicht nachgewiesen werden kann. Bezogen auf die Unfallrate, ist kein Unterschied nachweisbar.

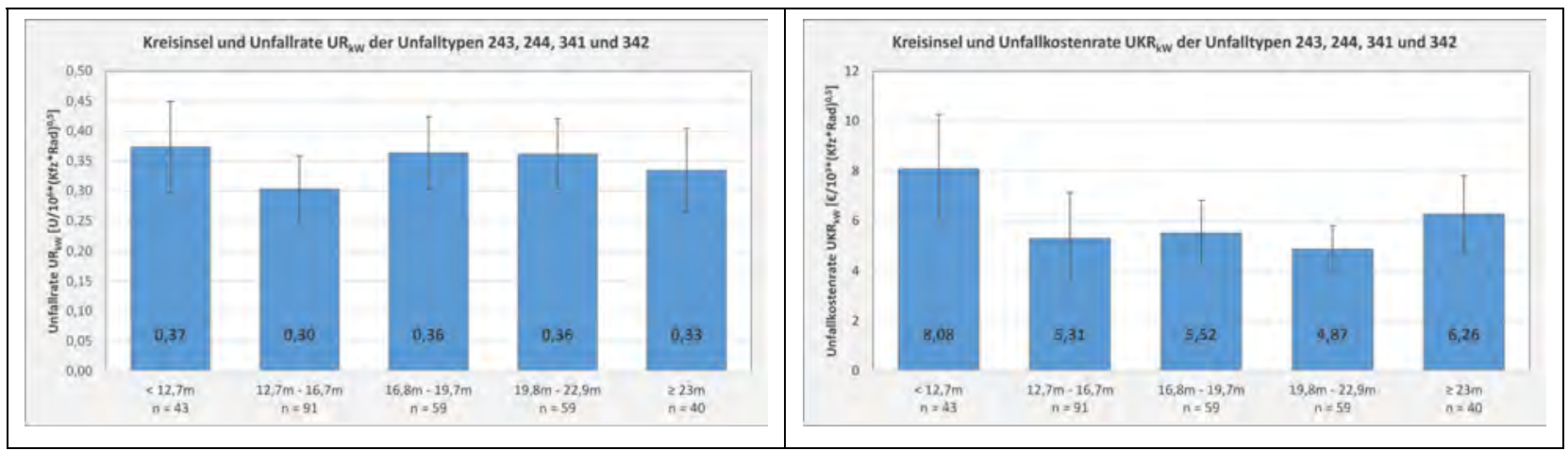

Abbildung 19: Durchmesser der Kreisinsel

Die Auswertung zeigt, dass der Durchmesser der Kreisinsel insbesondere die Schwere der Unfälle des Typs 244 (Kraftfahrer biegt aus, Radfahrer kommt von links) in den Ausfahrten beeinflusst. Die Unfallkostenrate für diesen Unfalltyp liegt an Kreisverkehren mit Kreisinseldurchmessern unter 12,7 m 
deutlich über den Werten bei größeren Außendurchmessern. Die $95 \%$-Konfidenzintervalle überschneiden sich jedoch, so dass eine Signifikanz der Unterschiede nicht nachgewiesen werden kann. Bezogen auf die Unfallrate zeigt sich kein Zusammenhang.

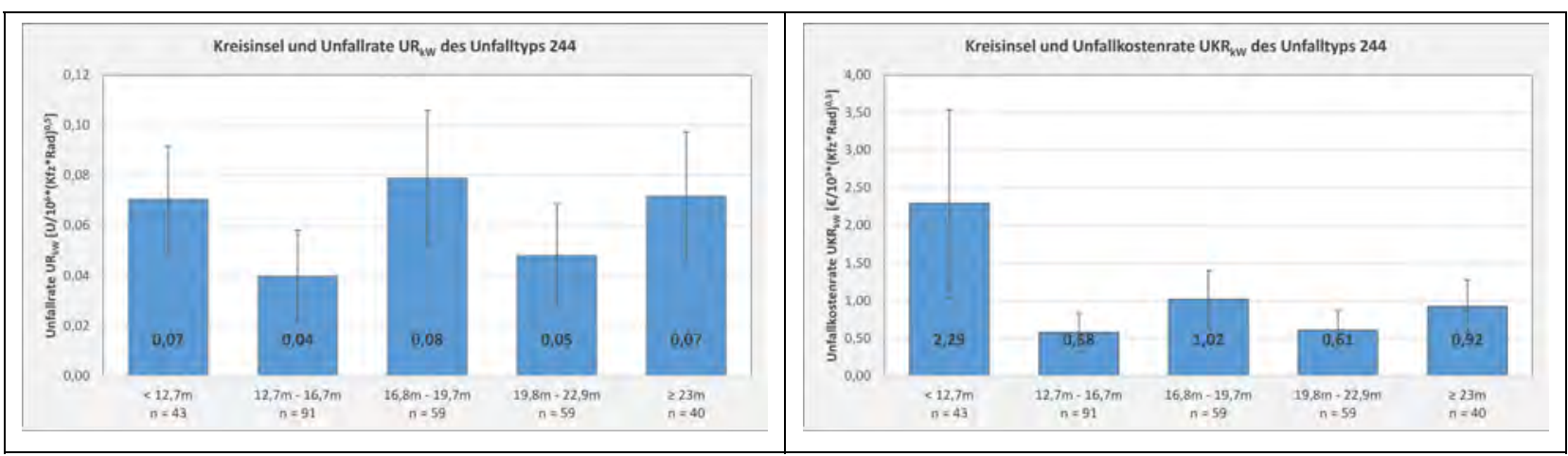

Abbildung 20: Durchmesser der Kreisinsel und Unfälle des Typs 244 in den Ausfahrten

\subsection{Breite des Innenrings}

Der Innenring wird insbesondere bei Kreisverkehren mit kleineren Außendurchmessern und demzufolge breiteren Kreisfahrbahnen eingesetzt, um die Geschwindigkeit der Kraftfahrzeuge beim Befahren der Kreisfahrbahn zu reduzieren. Es kann davon ausgegangen werden, dass bei kleineren Außendurchmessern breitere Innenringe erforderlich werden.

Die Kreisverkehre wurden nach der Breite des Innenrings in die folgenden Klassen eingeteilt:

- Kein Innenring

- 1,3 bis $1,9 \mathrm{~m}$

- 2,0 bis $2,4 \mathrm{~m}$

- 2,5 bis $2,9 \mathrm{~m}$

- 3 m und mehr

Die Gestaltung des Innenrings geht in diese Betrachtung nicht ein.

Bei Betrachtung aller Unfalltypen zeigt sich, dass die Kreisverkehrsarme an Kreisverkehren ohne Innenring die günstigsten Unfallkenngrößen aufweisen. Bei diesen Kreisverkehren kann davon ausgegangen werden, dass auch ohne Innenring eine ausreichende Ablenkung der einfahrenden Kraftfahrzeuge erreicht werden kann und ein Innenring daher nicht erforderlich ist. Kreisverkehre mit besonders breiten Innenringen weisen hingegen bezogen auf die Unfallschwere die ungünstigsten Werte auf. 


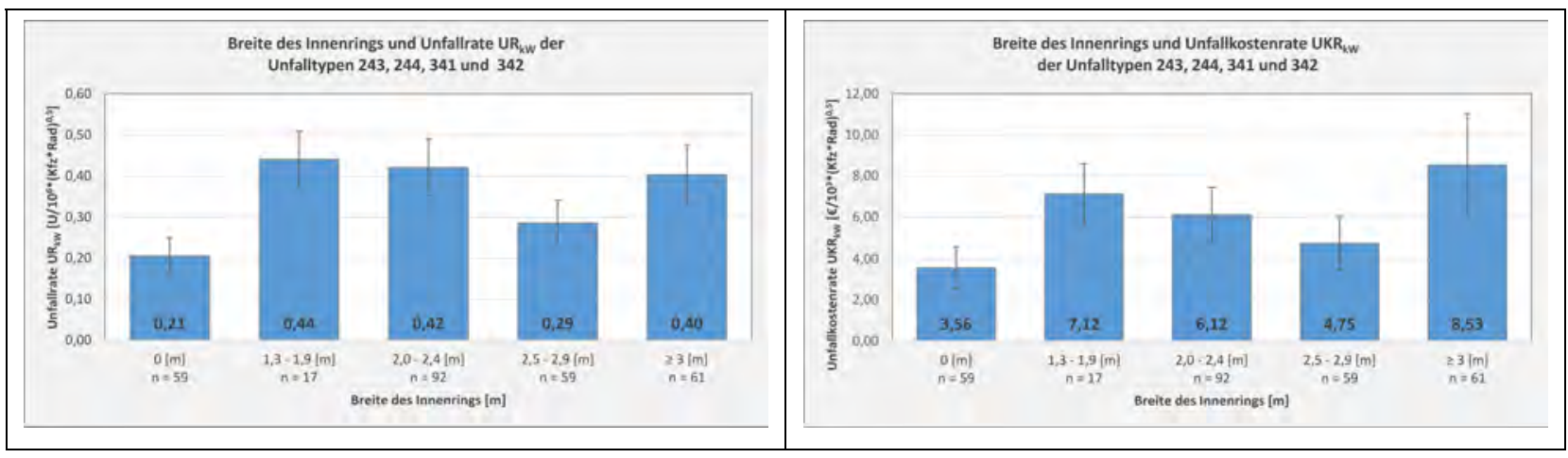

Abbildung 21: Breite des Innenrings

Die Breite des Innenrings beeinflusst in erster Linie die Unfallschwere in den Ausfahrten. Die folgende Abbildung zeigt die Zusammenhänge zwischen der Breite des Innenrings und den Unfällen des Typs 243 (Kraftfahrer biegt aus, Radfahrer kommt von rechts) und 244 (Kraftfahrer biegt aus, Radfahrer kommt von links) in den Ausfahrten. Bei einer Innenringbreite von $3 \mathrm{~m}$ und mehr steigt die Unfallkostenkostenrate massiv an.

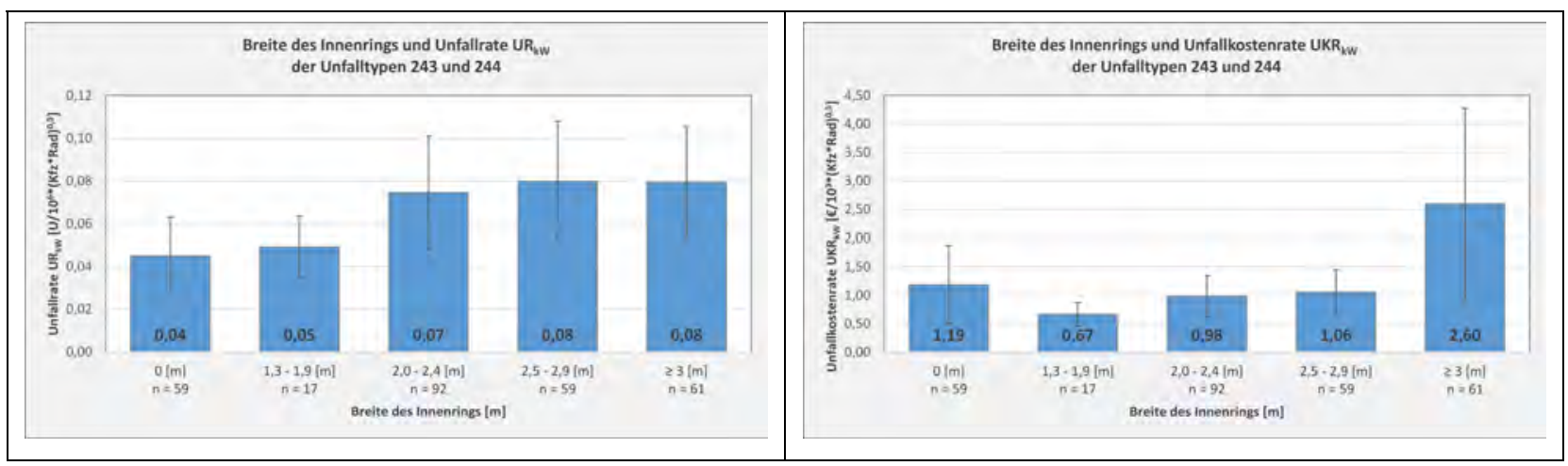

Abbildung 22: Breite des Innenrings und Unfälle der Typen 243 und 244 in den Ausfahrten

\subsection{Gestaltung des Innenrings}

Hinsichtlich der Gestaltung des Innenrings wurden die folgenden Kategorien gewählt:

- Kein Innenring

- Markiert

- Baulich, ohne Überhöhung zur asphaltierten Kreisfahrbahn

- Baulich, mit Überhöhung zwischen $1 \mathrm{~cm}$ und 2,4 cm

- Baulich, mit Überhöhung zwischen $2,5 \mathrm{~cm}$ und $3,9 \mathrm{~cm}$

- Baulich, mit Überhöhung ab $4 \mathrm{~cm}$ 
Es zeigt sich, dass die Kreisverkehrsarme der Kategorien „kein Innenring“ und „baulich gestaltet ohne Überhöhung" die günstigsten Unfallkenngrößen aufweisen. Bei den Kreisverkehren ohne Innenring kann davon ausgegangen werden, dass auch ohne Innenring eine ausreichende Ablenkung der einfahrenden Kraftfahrzeuge erreicht werden kann und ein Innenring daher nicht erforderlich ist. Bei den Kreisverkehren mit baulich gestaltetem Innenring, ohne Überhöhung sind die Innenringe zumeist sehr rau gestaltet oder haben eine größere Querneigung als die asphaltierte Kreisfahrbahn.

Die folgende Abbildung zeigt unterschiedliche Gestaltungsvarianten.

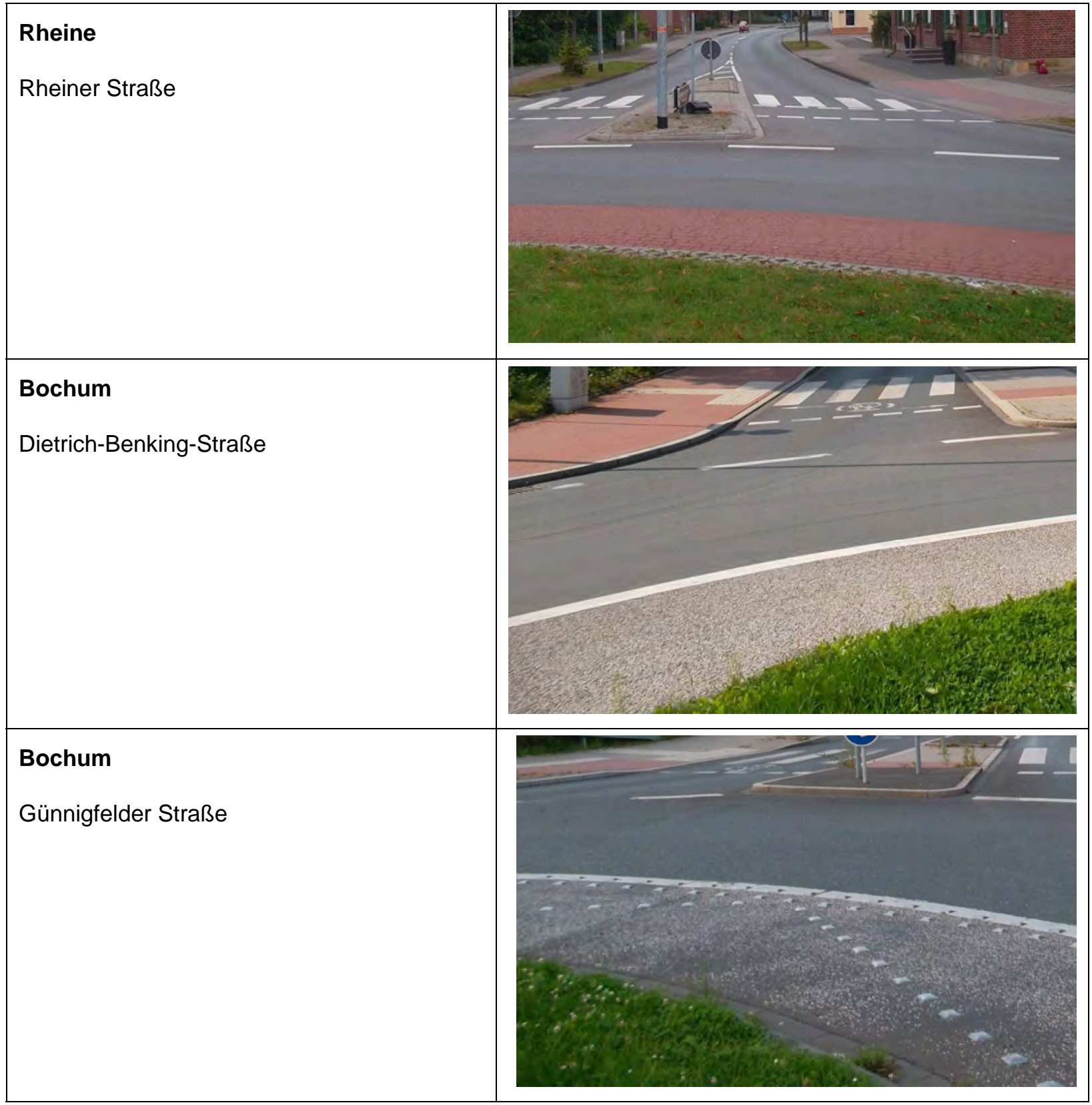

Abbildung 23: Innenringe in rauer Ausbildung und/oder größere Querneigung

Die deutlich ungünstigsten Unfallkenngrößen weisen die Kreisverkehrsarme an Kreisverkehren mit ausschließlich markiertem Innenring sowie mit baulich gestaltetem, aber nur geringfügig überhöhtem Innenring (bis 2,4 cm) auf. 


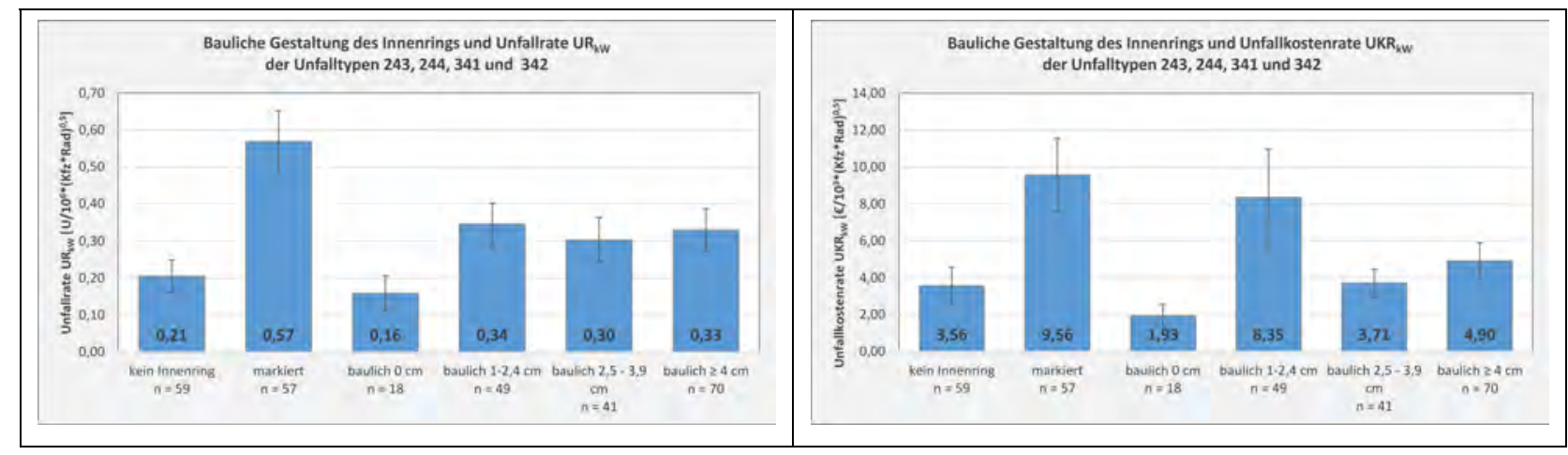

Abbildung 24: Gestaltung des Innenrings

\subsection{Breite der Kreisfahrbahn}

Die Untersuchungen zeigen, dass eine Betrachtung der gesamten Kreisfahrbahn zu keinen signifikanten Unterschieden bei den Unfallkenngrößen führt. In einem nächsten Schritt wurde nur der Teil der Kreisfahrbahn betrachtet, der von Pkw ohne nennenswerte Komforteinbußen befahren werden kann. Dabei wurden die Innenringe, die entweder besonders rau gestaltet waren oder eine besonders hohe Absetzung von der asphaltierten Kreisfahrbahn aufwiesen $(>4 \mathrm{~cm})$, aus der Kreisfahrbahnbreite herausgerechnet.

Die folgende Abbildung zeigt, dass bei einer komfortabel befahrbaren Kreisfahrbahnbreite ab 7,5 m eine deutliche Erhöhung der Unfallkenngrößen, insbesondere der Unfallkostenrate auftritt. Die Unfallkostenrate liegt etwa $60 \%$ über den Werten für Kreisverkehre mit komfortabel befahrbaren Kreisfahrbahnen unter $7,5 \mathrm{~m}$.

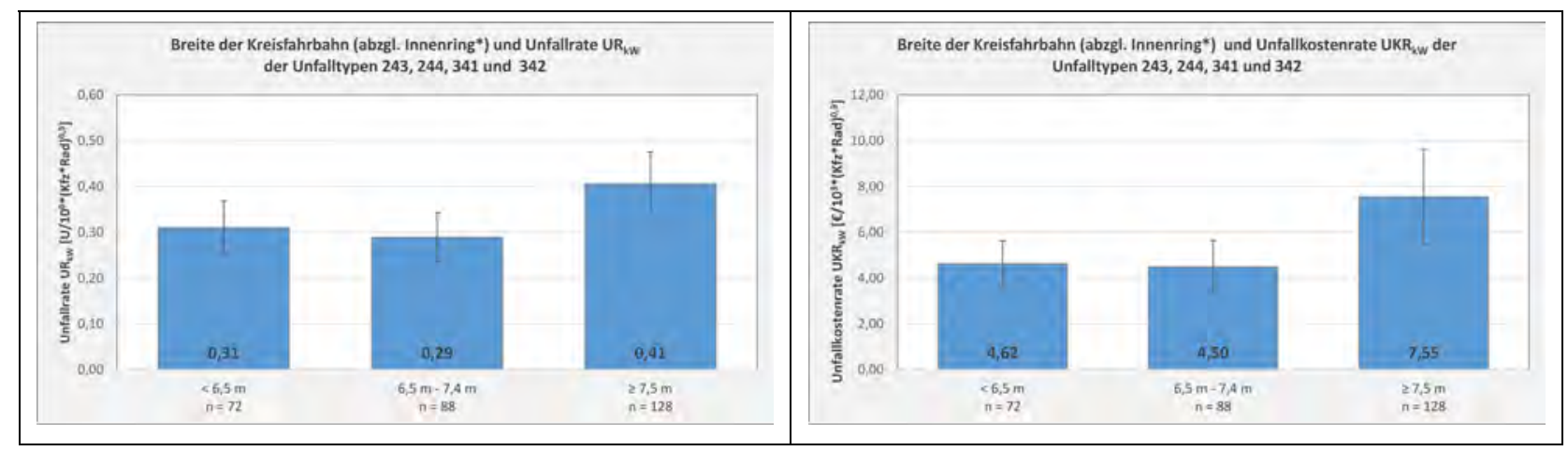

Abbildung 25: Breite der Kreisfahrbahn und Unfälle des Unfalltyps 243, 244, 341 und 342

Besonders auffällig sind die Unterschiede in den Ausfahrten. Die Unfallkostenrate beträgt mehr als das Doppelte der Unfallkostenrate von Kreisverkehren mit schmaleren Kreisfahrbahnen. 


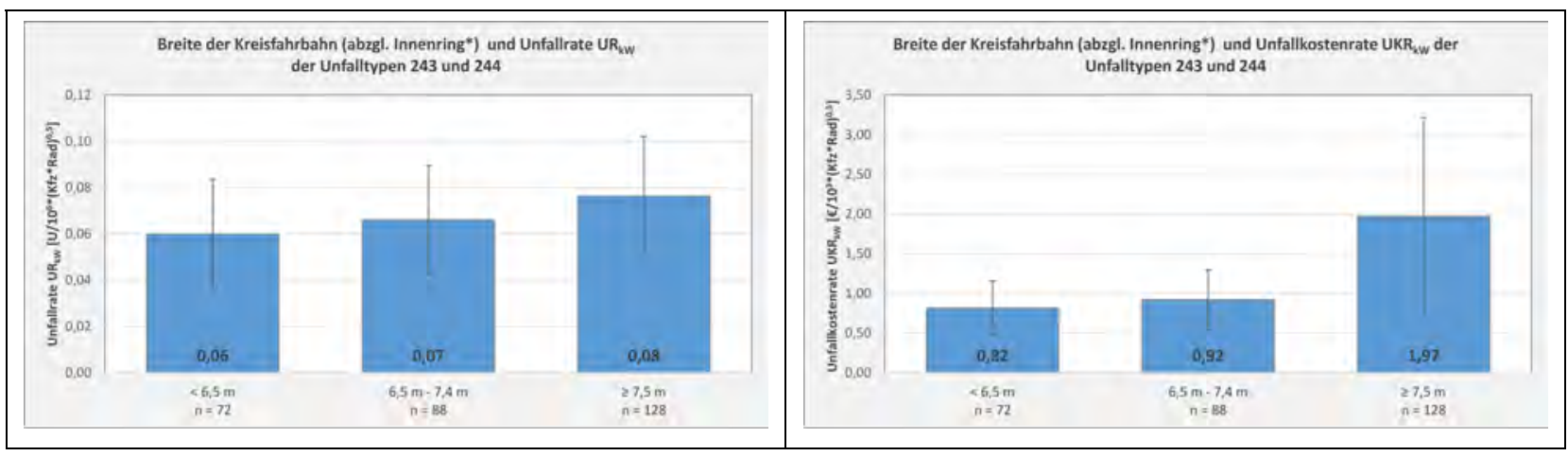

Abbildung 26: Breite der Kreisfahrbahn und Unfälle in den Ausfahrten

In den Zufahrten fällt der Unterschied nicht ganz so deutlich aus. Die Unfallkostenrate liegt etwa $25 \%$ über der Unfallkostenrate von Kreisverkehren mit schmaleren Kreisfahrbahnen.

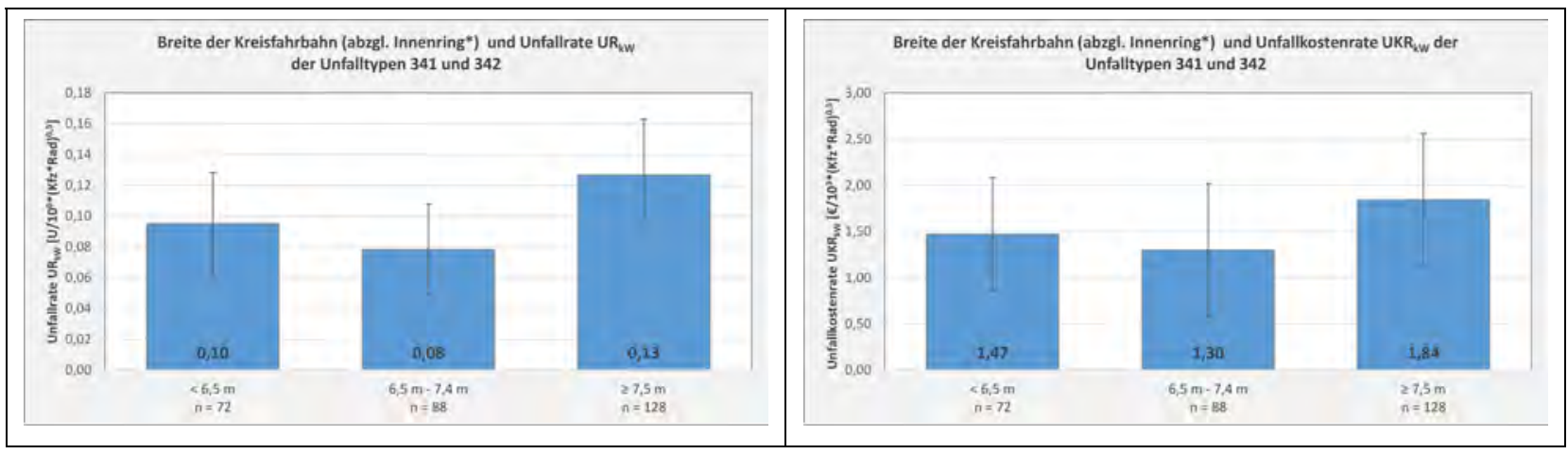

Abbildung 27: Breite der Kreisfahrbahn und Unfälle in den Zufahrten

\subsection{Kunst auf der Kreisinsel}

Das Vorhandensein von Kunstobjekten auf der Kreisinsel hat bezogen auf alle Unfälle keinen signifikanten Einfluss auf das Unfallgeschehen mit Radfahrern. Die Unfallkenngrößen an Kreisverkehrsarmen mit Kunstobjekten auf der Kreisinsel liegen zwar um etwa $15 \%$ über den Werten für Kreisverkehrsarme ohne Kunstobjekte, doch ist der Unterschied aufgrund der sich stark überschneidenden Konfidenzintervalle nicht signifikant.
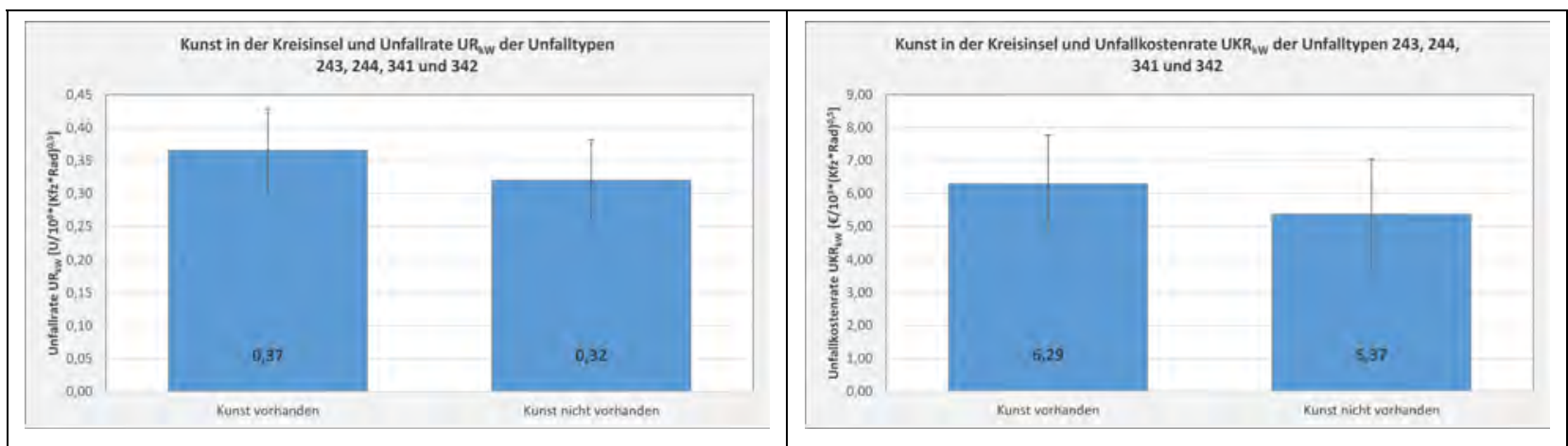

Abbildung 28: Kunst auf der Kreisinsel 
Es kann angenommen werden, dass Kunst im Kreis ausschließlich die Aufmerksamkeit der in den Kreisverkehr einfahrenden Kraftfahrer beeinträchtigt. Die folgende Abbildung zeigt, dass bezogen auf den Unfalltyp 341 (Kraftfahrer biegt ein, Radfahrer kommt von links) deutlichere Unterschiede auftreten. Die Unfallkostenrate bei Kreisverkehren mit Kunst auf der Kreisinsel liegt etwa $60 \%$ über der Unfallkostenrate bei Kreisverkehren ohne Kunstobjekte.

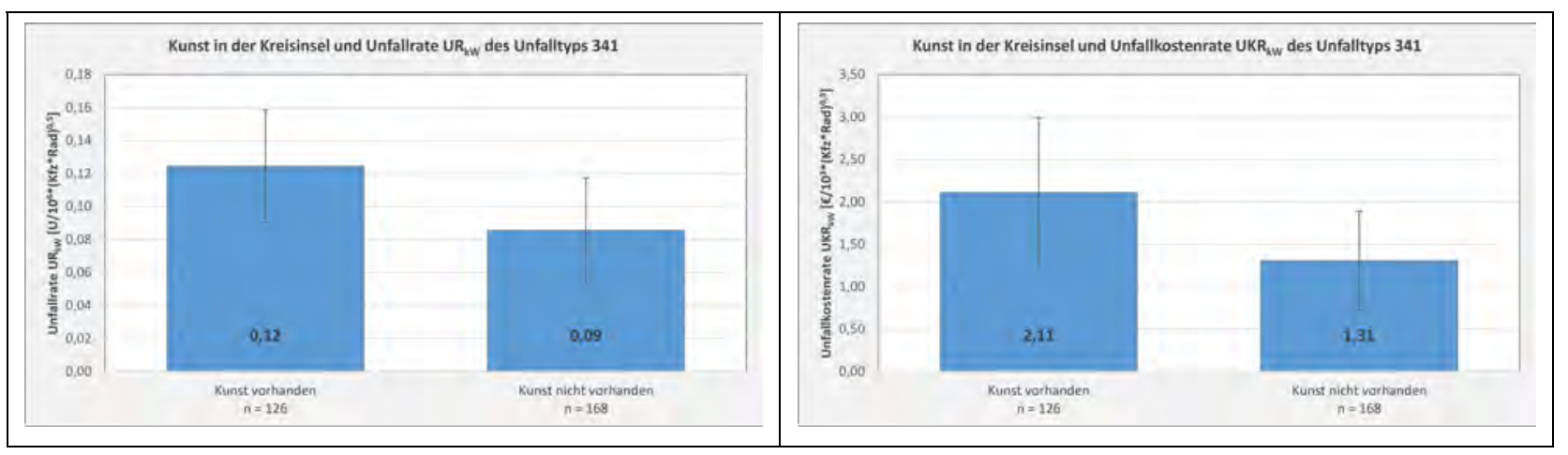

Abbildung 29: Kunst auf der Kreisinsel und Unfälle des Typs 341

\subsection{Führung des Radverkehrs}

Es besteht ein Zusammenhang zwischen dem Unfallgeschehen und der Art der Radverkehrsführung:

- Radweg

- Gemeinsamer Geh-/Radweg

- Gehweg, Radfahrer frei

Bezogen auf die Unfallkostenrate, bei der die Unfallschwere berücksichtigt wird, erweisen sich gemeinsame Geh-/Radwege sowie Gehwege mit dem Zusatz „Radfahrer frei“ als etwas sicherer als separate Radwege.
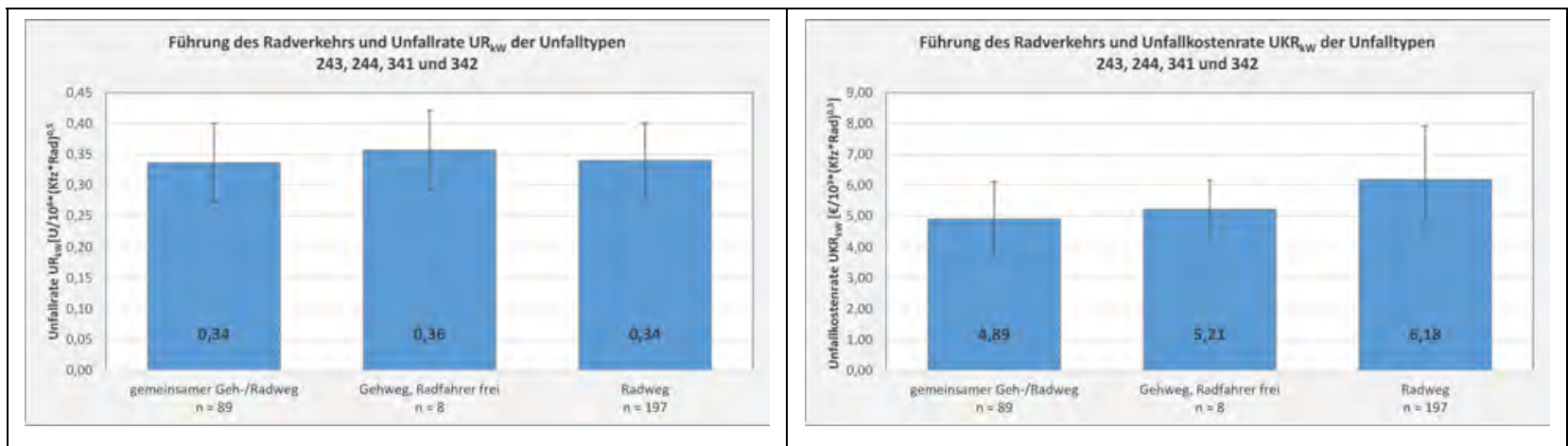

Abbildung 30: Führung des Radverkehrs 


\subsection{Führung des Radverkehrs in der Zufahrt}

Es wird zwischen den folgenden Führungsformen unterschieden:

- Der Radfahrer wird in der Zufahrt auf der Fahrbahn geführt und erst vor dem Kreisverkehr auf die Nebenanlagen geführt.

- Der Radweg wird bereits in der Zufahrt auf den Nebenanlagen geführt.

Die folgenden Abbildungen verdeutlichen die Führungsformen.

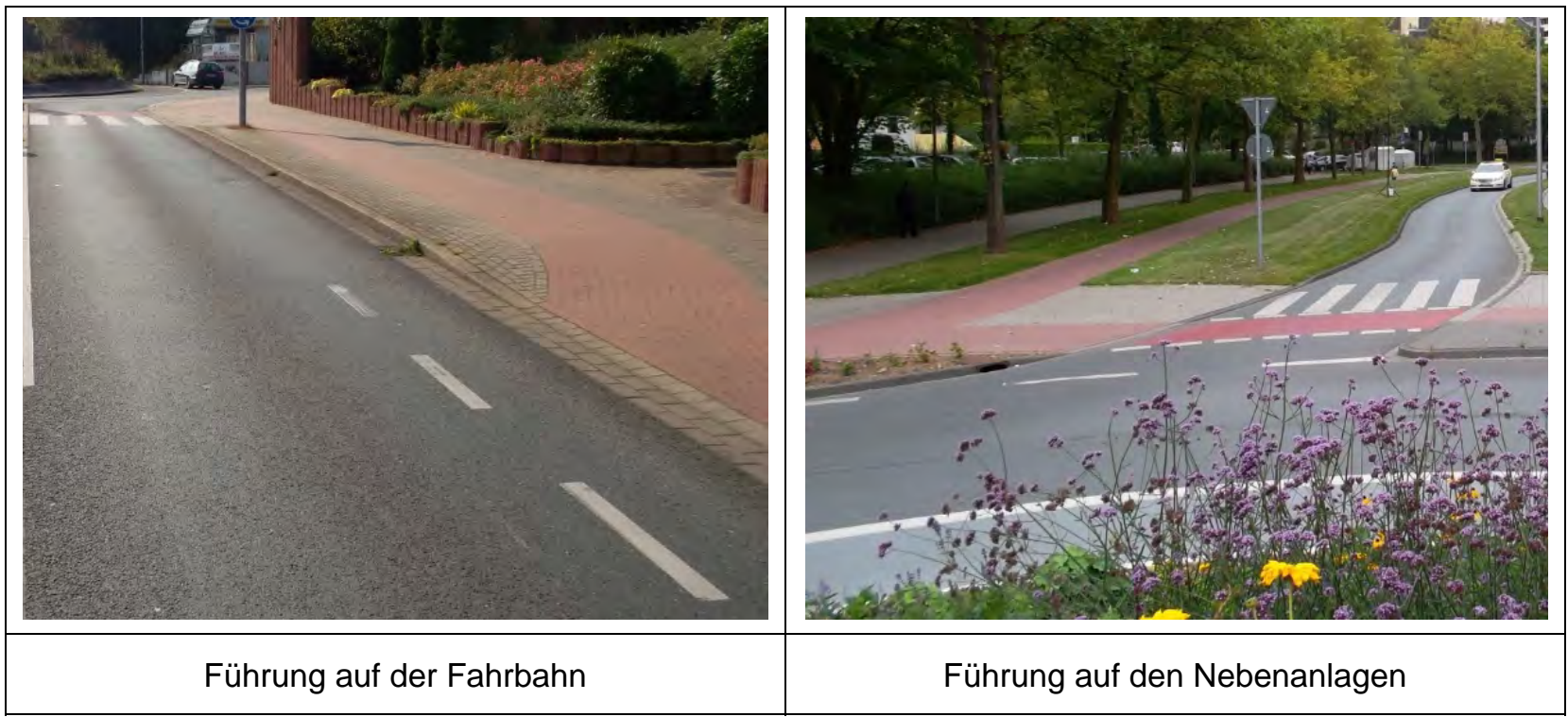

Abbildung 31: Führung des Radverkehrs in der Zufahrt

Es zeigt sich, dass Kreisverkehrsarme mit Führung der Radfahrer in den Zufahrten auf der Fahrbahn deutlich günstigere Unfallkenngrößen aufweisen.
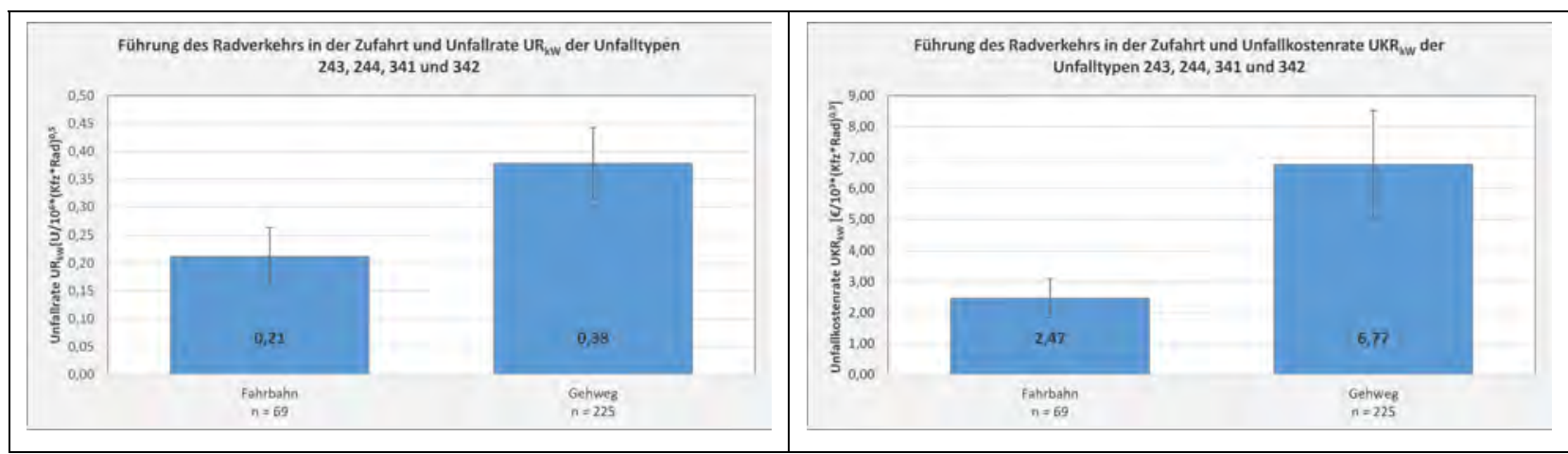

Abbildung 32: Führung des Radverkehrs in der Zufahrt 


\subsection{Ein- und Zweirichtungsverkehr}

Es zeigt sich, dass Kreisverkehre mit Zweirichtungsradwegen etwas ungünstigere Unfallkenngrößen aufweisen, als Kreisverkehre mit Einrichtungsradwegen.
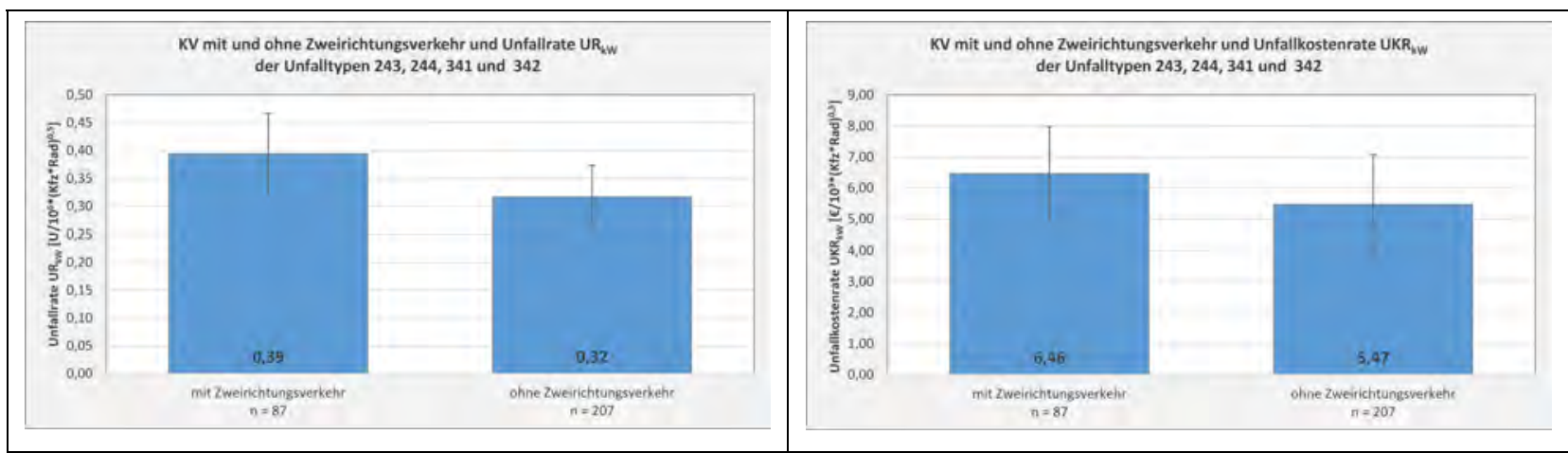

Abbildung 33: Ein- und Zweirichtungsradverkehr

Signifikante Unterschiede zeigen sich beim Unfalltyp 244 (Kraftfahrer biegt aus dem Kreisverkehr aus, Radfahrer kommt von links). Die Unfallkostenrate liegt für diesen Unfalltyp an Kreisverkehren mit Zweirichtungsverkehr viermal höher als bei den Kreisverkehren mit Einrichtungsverkehr. Bei den übrigen Unfalltypen sind keine signifikanten Unterschiede erkennbar.

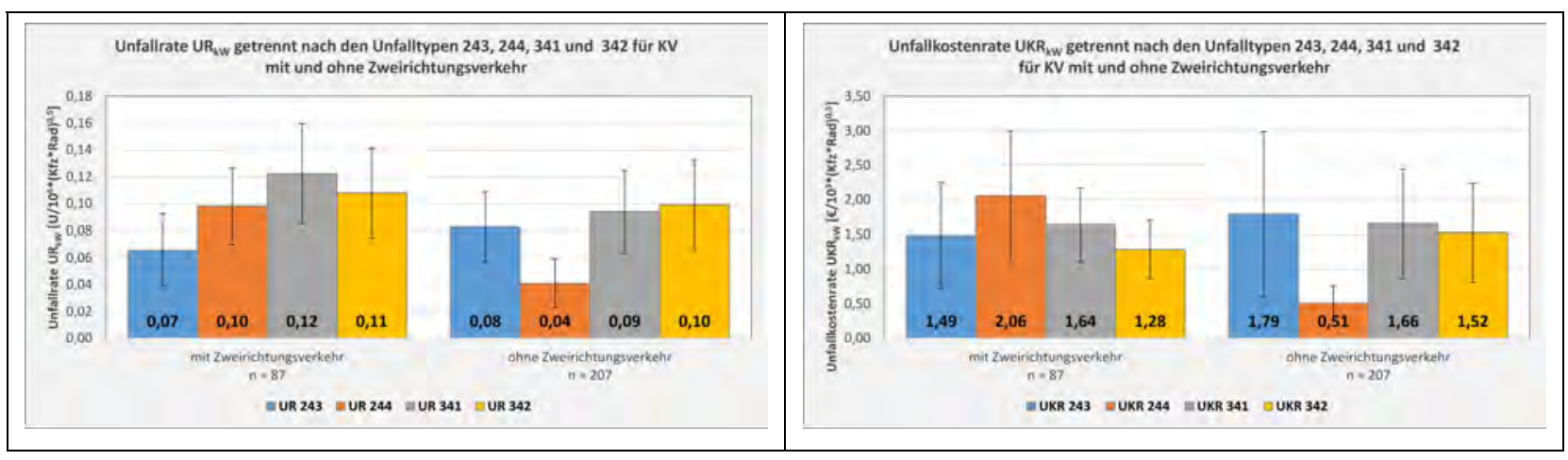

Abbildung 34: Ein- und Zweirichtungsradverkehr, differenziert nach Unfalltypen

Das Ergebnis ist unabhängig von der Anzahl der Radfahrer im Uhrzeigersinn. Die folgende Abbildung zeigte eine Differenzierung nach der Anzahl der im Uhrzeigersinn fahrenden Radfahrer. Dabei wird deutlich, dass die Unfallkenngrößen bei Zweirichtungsverkehr in allen Belastungsklassen deutlich höher liegen als bei Einrichtungsverkehr. 


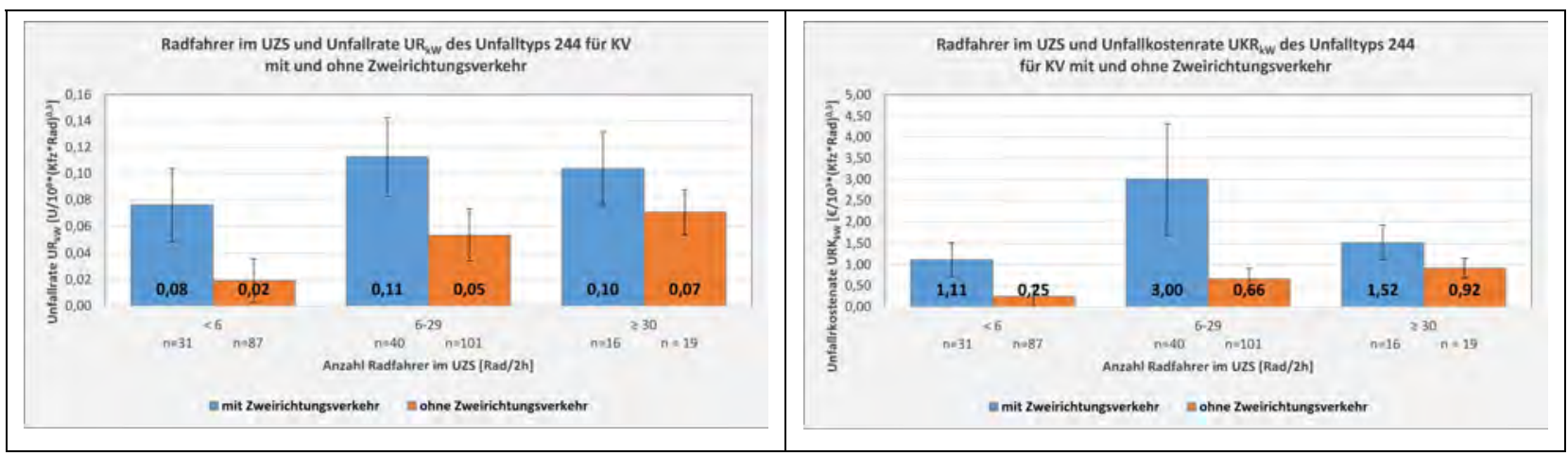

Abbildung 35: Ein- und Zweirichtungsradverkehr, Unfalltypen 244 differenziert nach Belastungsklassen

\subsection{Furteinfärbung}

Kreisverkehrsarme mit eingefärbten Radfahrerfurten weisen ein höheres Unfallrisiko für Radfahrer auf als Kreisverkehre ohne Furteinfärbung. Bei der Bewertung dieses Zusammenhangs ist allerdings zu berücksichtigen, dass unfallauffällige Kreisverkehre möglicherweise besonders häufig mit eingefärbten Furten ausgestattet werden. Eingefärbte Furten sind daher nicht zwangsläufig die Ursache für ein höheres Unfallrisiko. Als Maßnahme zur Reduzierung der Unfälle mit Radfahrern ist diese Maßnahme hingegen ungeeignet.
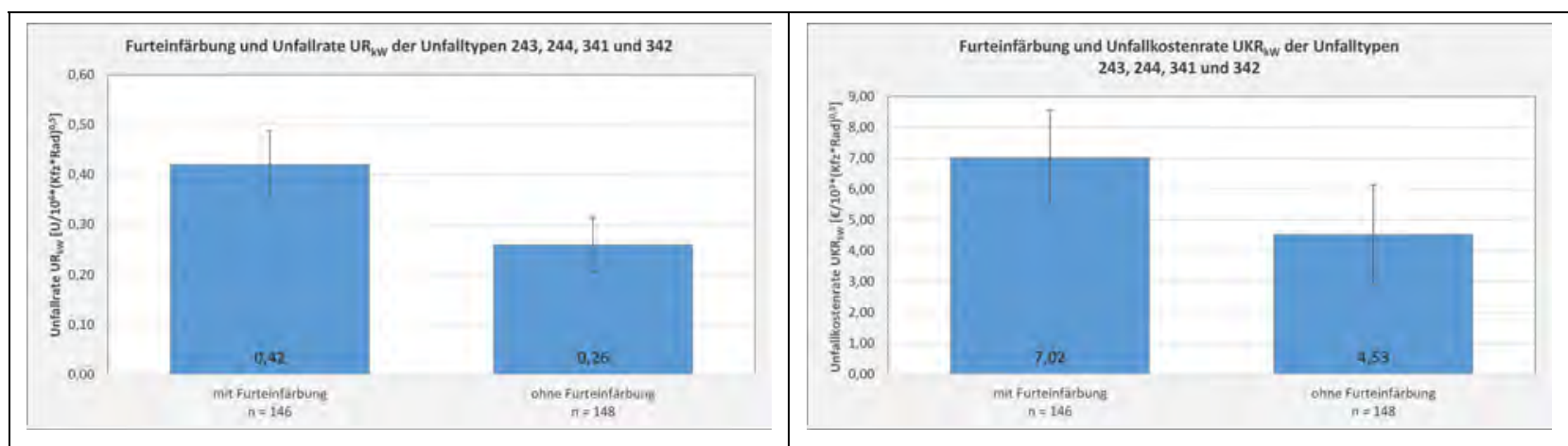

Abbildung 36: Furteinfärbung 


\subsection{Furten und Piktogramme}

Bezogen auf alle Kreisverkehrsarme hat das Aufbringen von Radfahrerpiktogrammen keinen positiven Einfluss auf die Verkehrssicherheit von Radfahrern. Tendenziell weisen Radfahrerfurten ohne Piktogramme ein geringeres Unfallrisiko für Radfahrer auf. Dieser Zusammenhang ist jedoch statistisch nicht signifikant.
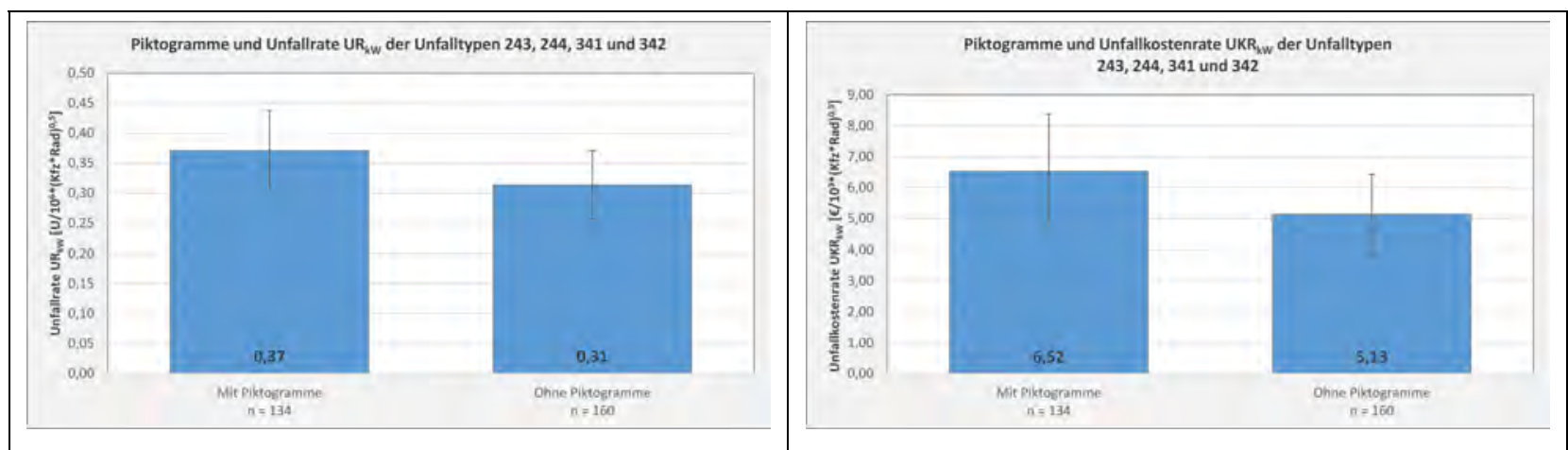

Abbildung 37: Piktogramme auf den Radfahrerfurten

Betrachtet man nur die Furten mit Zweirichtungsverkehr, so zeigt sich, dass das Aufbringen von Piktogrammen mit Fahrtrichtungsangabe einen deutlichen positiven Einfluss auf die Verkehrssicherheit der Radfahrer hat.

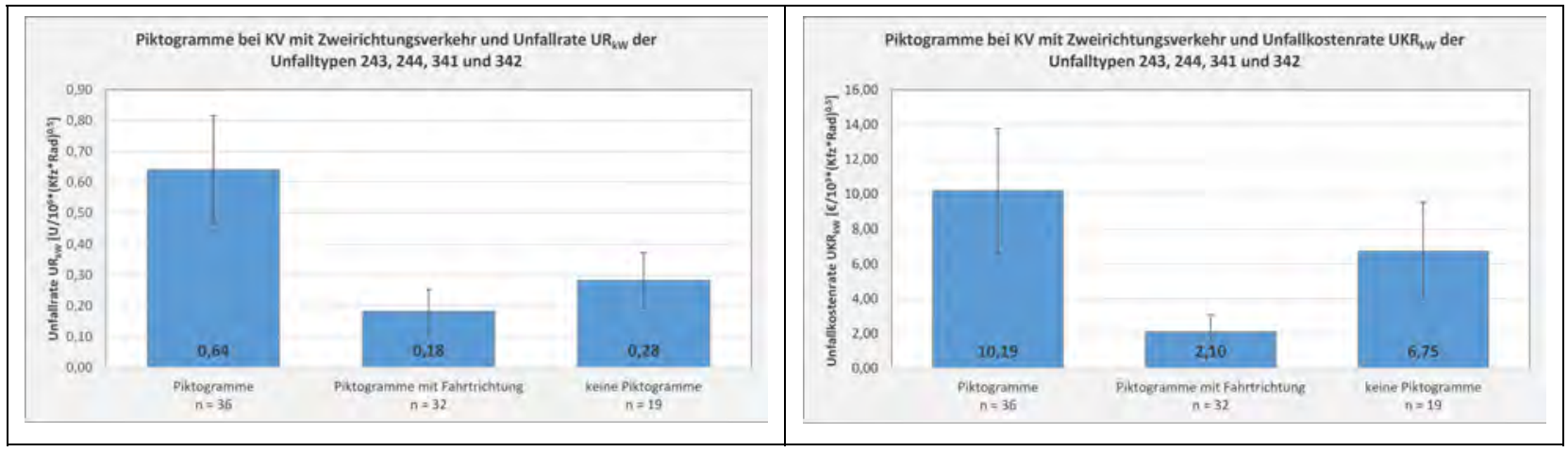

Abbildung 38: Piktogramme auf den Radfahrerfurten mit Zweirichtungsverkehr 


\subsection{Breite der Furt}

Bezogen auf die Breite der Radfahrerfurten zeigen sich bei den Furten mit einer Breite zwischen 1,50 und 2,40 m günstigere Unfallkenngrößen, als bei schmaleren oder breiteren Furten. Aufgrund der Überschneidung der Konfidenzintervalle ist dieser Zusammenhang jedoch nicht signifikant.
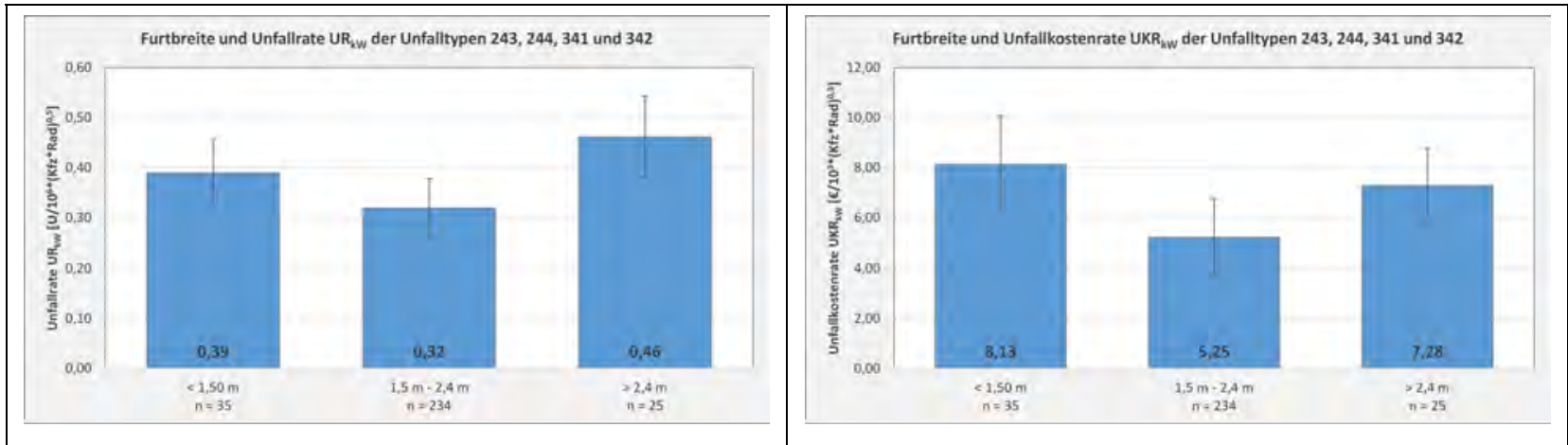

Abbildung 39: Breite der Radfahrerfurt

Die folgende Abbildung zeigt die Differenzierung nach Ein- und Zweirichtungsverkehr. Es wird deutlich, dass Furtbreiten unter $1,5 \mathrm{~m}$ bei Radwegen im Zweirichtungsverkehr zu deutlich höheren Unfallkennwerten führen. Bei Einrichtungsradwegen haben schmalere Furten hingegen einen positiven Einfluss auf die Verkehrssicherheit. Furten mit einer Breite von mehr als 2,4 m wirken sich unabhängig vom Ein- und Zweirichtungsverkehr eher negativ auf die Verkehrssicherheit aus.

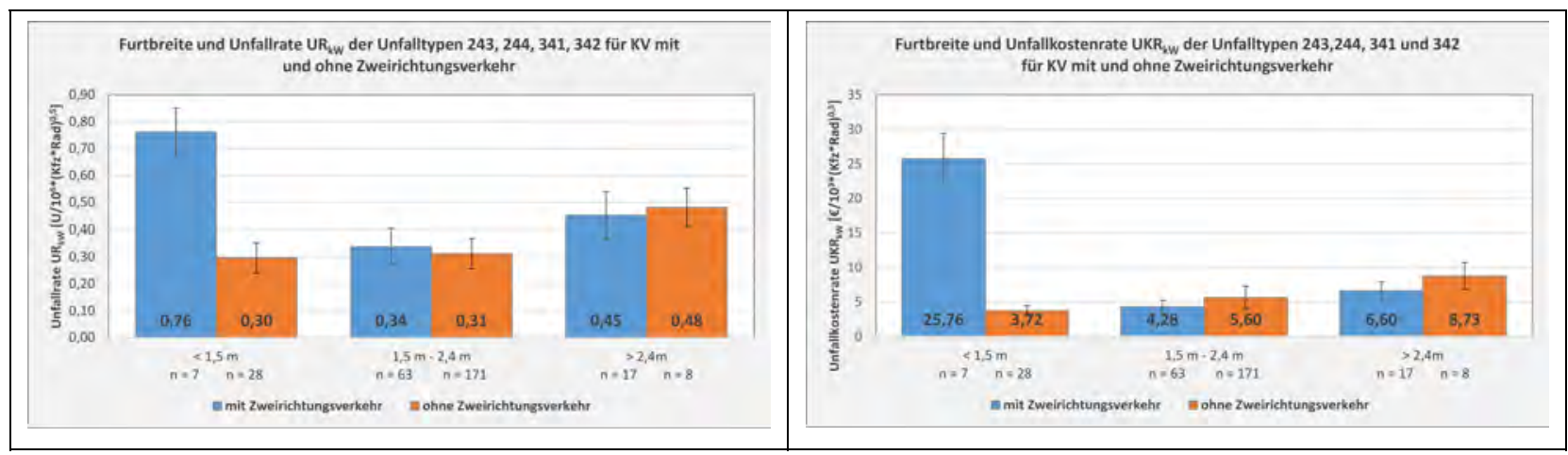

Abbildung 40: Breite der Radfahrerfurt für Kreisverkehre mit und ohne Zweirichtungsverkehr 


\subsection{Absetzung der Furt von der Kreisfahrbahn}

Bezogen auf alle Unfalltypen ist kein signifikanter Zusammenhang zwischen dem Unfallgeschehen und der Furtabsetzung erkennbar. Lediglich bezogen auf die Unfallrate weisen nur gering abgesetzte Furten $(<2,5 \mathrm{~m})$ ein höheres Unfallrisiko für Radfahrer auf.

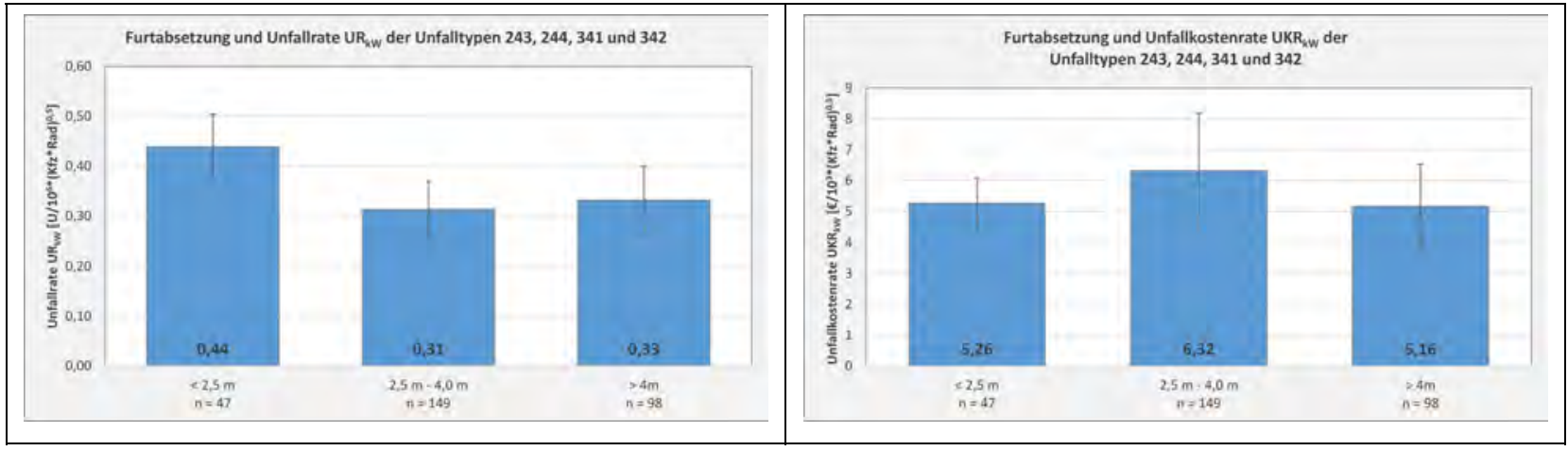

Abbildung 41: Furtabsetzung und Unfälle

Bezogen auf den Unfalltyp 243 (Kraftfahrer biegt aus, Radfahrer kommt von rechts) sind hingegen deutlichere Unterschiede erkennbar. Hier zeigen die Furten mit Absetzungen von über $4 \mathrm{~m}$ ein deutlich geringeres Unfallrisiko für Radfahrer.
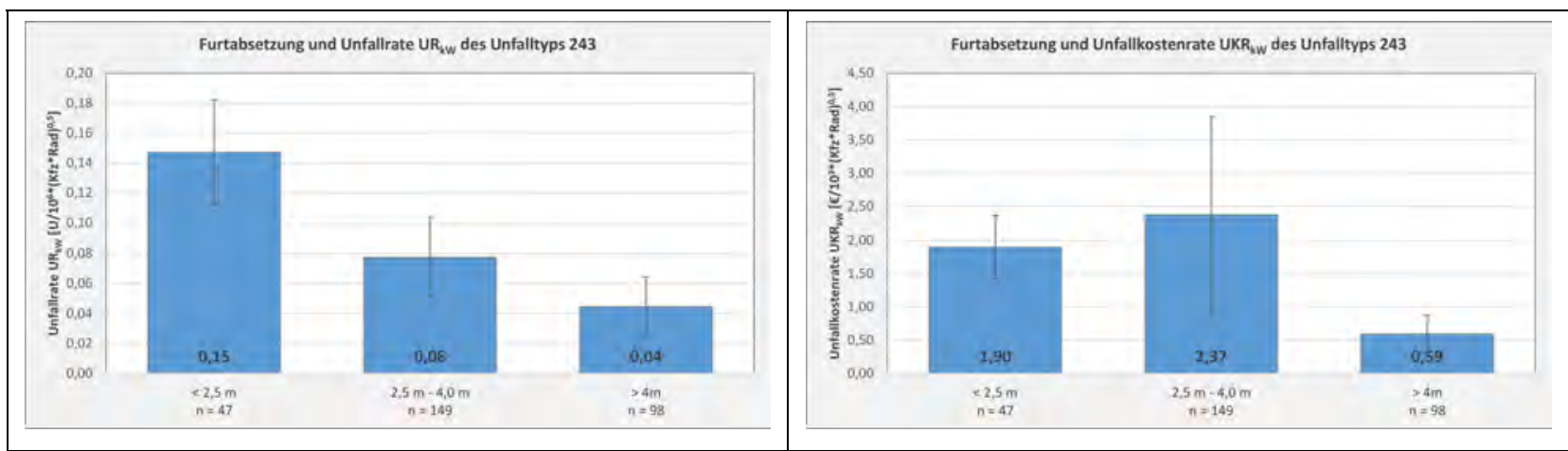

Abbildung 42: Furtabsetzung und Unfalltyp 243 


\subsection{Bewuchs der Beete}

Der Bewuchs der Beete zu den Zeitpunkten der Unfälle ist nicht rekonstruierbar. Ersatzweise wurde daher geprüft, inwieweit die zum Zeitpunkt der Ortsbesichtigung vorgefundenen Beete geeignet sind, höheren Bewuchs auszubilden. Die folgenden Abbildungen zeigen zwei Beispiele. Bei dem Beet auf dem rechten Bild kann ein höherer Bewuchs ausgeschlossen werden.

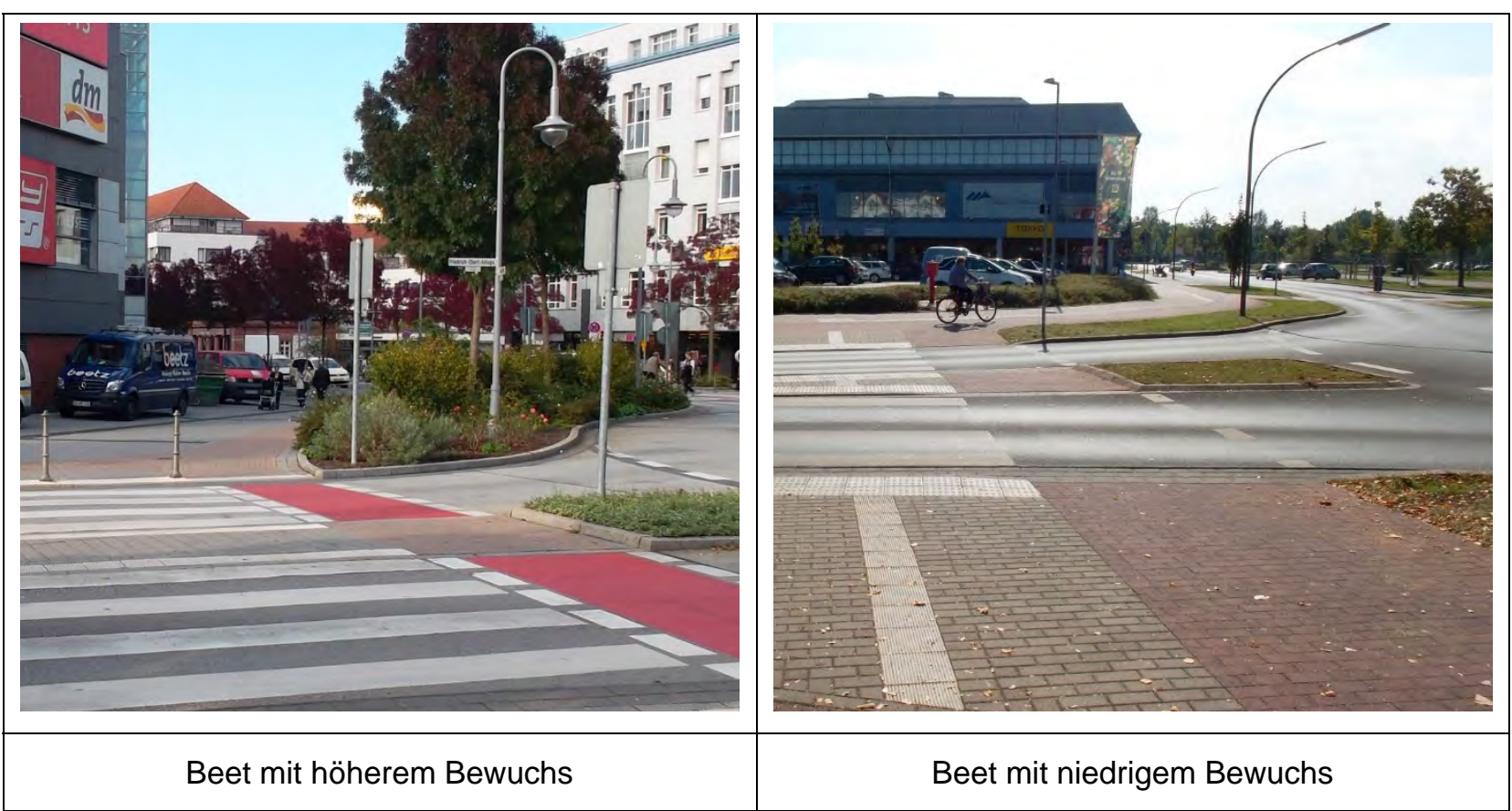

Abbildung 43: Bewuchs der Beete

Ein Zusammenhang zwischen dem Bewuchs der Beete und dem Unfallrisiko für Radfahrer ist nicht erkennbar.

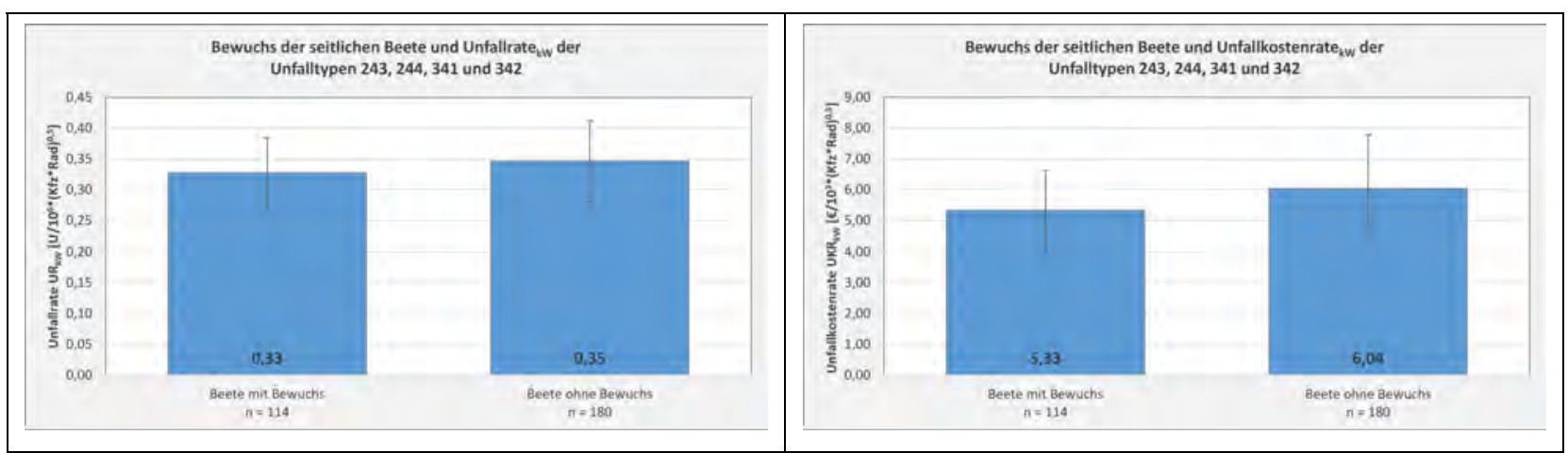

Abbildung 44: Bewuchs der Beete 


\subsection{Lage des Verzweigungspunktes des Radweges}

Es wurden an jedem Kreisverkehrsarm diejenigen Punkte definiert, an denen die tatsächliche Fahrtrichtung des Radfahrers für den Kraftfahrer erkennbar wird. Dabei wurde der Abstand dieses Verzweigungspunktes vom Fahrbahnrand gemessen. Die folgende Abbildung zeigt die Definition der Verzweigungspunkte.

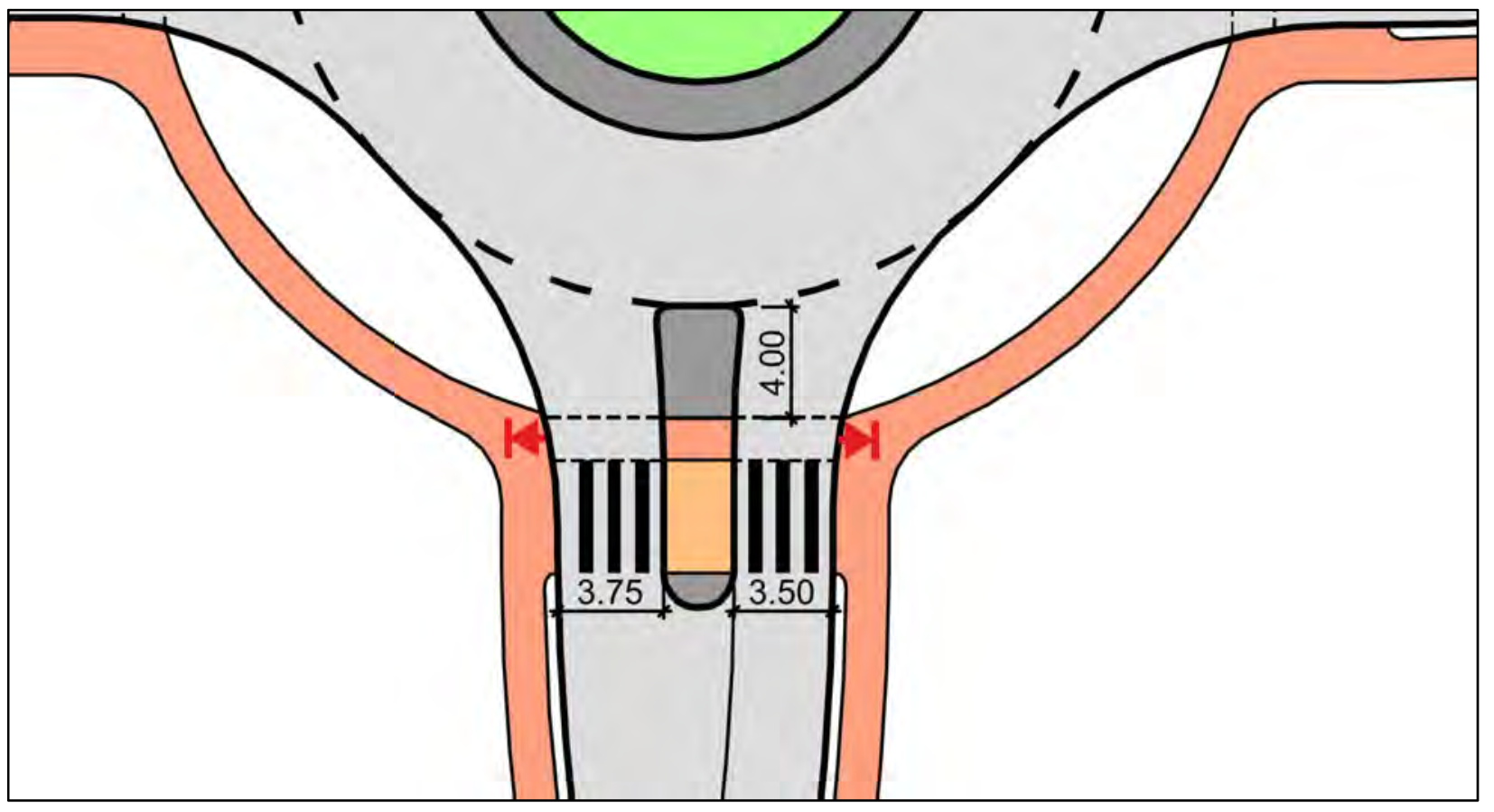

Abbildung 45: Definition der Verzweigungspunkte

Es ist anzunehmen, dass die Lage des Verzweigungspunktes vor der Ausfahrt die Erkennbarkeit der Fahrtrichtung für den Kraftfahrer beeinflusst. Es zeigt sich, dass bei einem besonders weit entfernten Verzweigungspunkt (über $4 \mathrm{~m}$ ) eine deutliche Zunahme des Unfalltyps 243 (Kraftfahrer biegt aus, Radfahrer kommt von rechts) auftritt. Die günstigsten Werte werden bei einer sehr kreisnahen Führung erreicht.
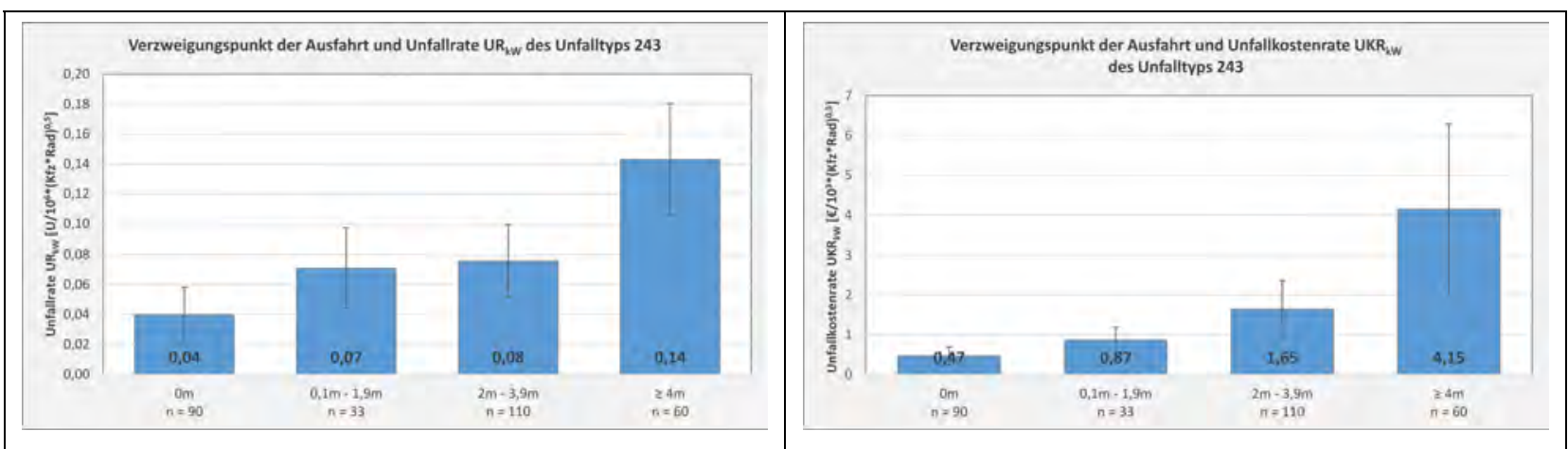

Abbildung 46: Lage des Verzweigungspunktes in der Ausfahrt und Unfalltyp 243 
Ebenso ist anzunehmen, dass die Lage des Verzweigungspunktes vor der Zufahrt die Erkennbarkeit der Fahrtrichtung für den einfahrenden Kraftfahrer beeinflusst. Hier zeigt sich aber kein Zusammenhang zwischen dem Unfalltyp 342 (Kraftfahrer biegt ein, Radfahrer kommt von rechts) und der Lage des Entscheidungspunktes.

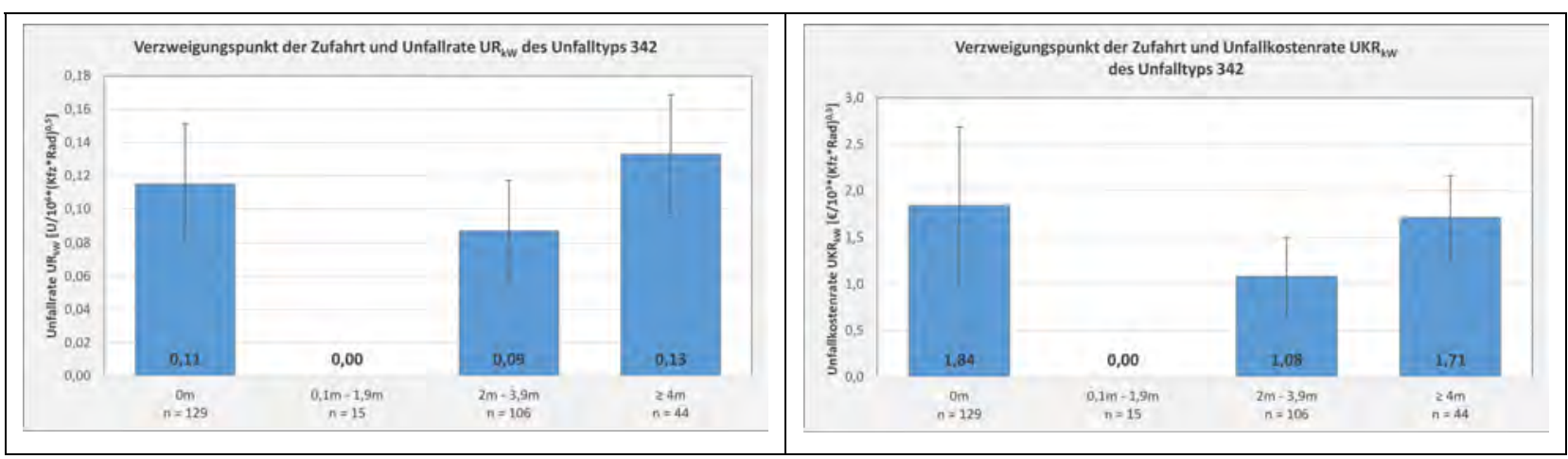

Abbildung 47: Lage des Verzweigungspunktes in der Zufahrt und Unfalltyp 342

\subsection{Abstand des Radweges zum Kreisrand}

Der Abstand des Radwegs vom Kreisrand beeinflusst die Erkennbarkeit des Radfahrers für den aus der Kreisfahrbahn ausfahrenden Kraftfahrer. Ein geringer Abstand begünstigt die Erkennbarkeit. Für die Auswertungen wurde der maximale Abstand des Radwegs von der Kreisfahrbahn im Quadranten vor der Kreisverkehrsausfahrt gemessen. Die folgende Abbildung zeigt die jeweils relevante Messstelle.

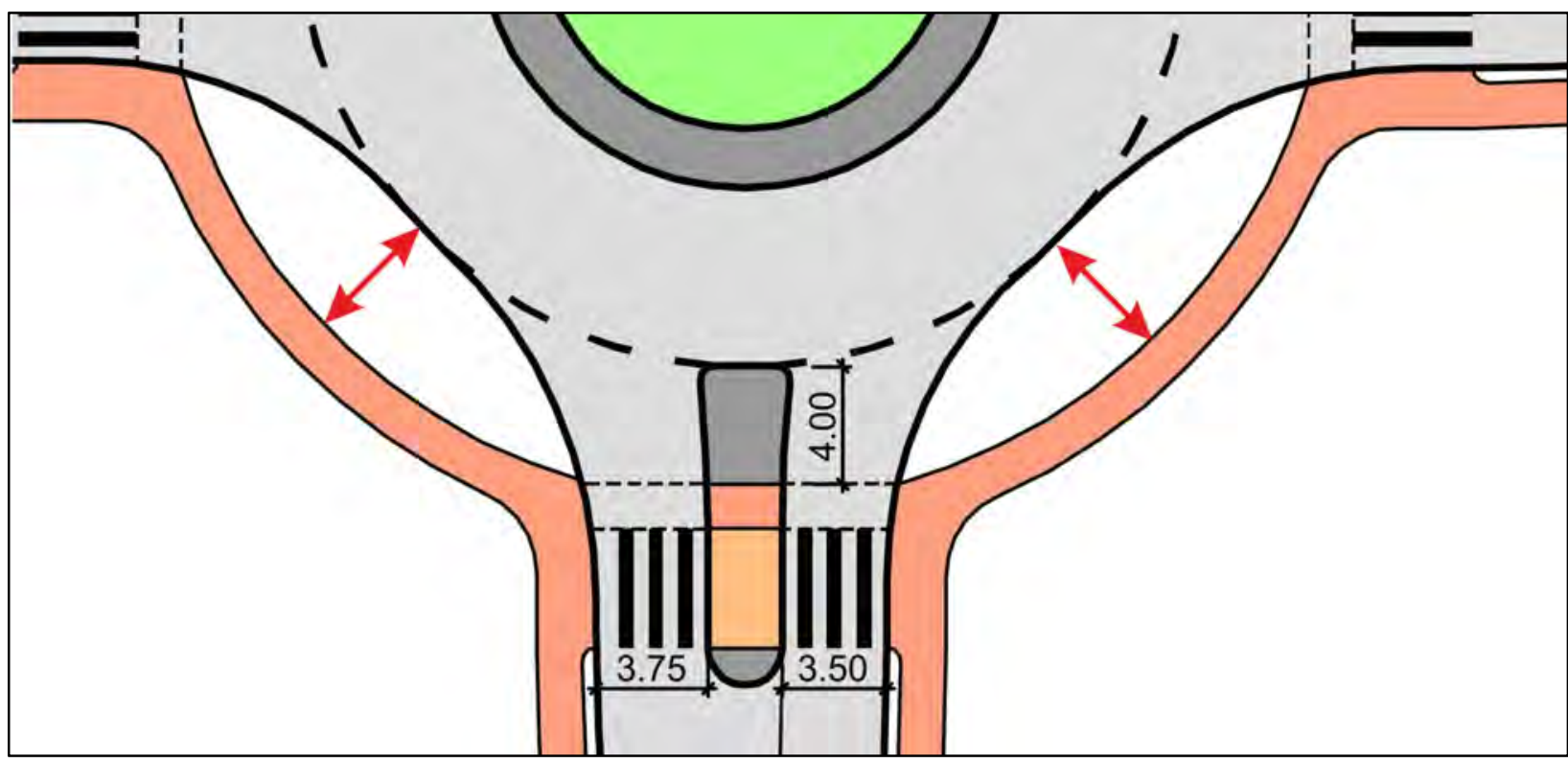

Abbildung 48: Abstand des Radweges zum Kreistand

Es wurden nur die Unfälle des Typs 243 (Kraftfahrer biegt aus, Radfahrer kommt von rechts) berücksichtigt. Es zeigt sich, dass bei Absetzungen von $4 \mathrm{~m}$ und mehr das Aufkommen an Unfällen des Typs 243 massiv ansteigt. Die auf den Unfalltyp 243 bezogen besonders günstige Unfallkenngrößen treten bei eng anliegenden Radwegen verbunden mit einer winkligen Führung des Radweges um den Kreisverkehr auf. 


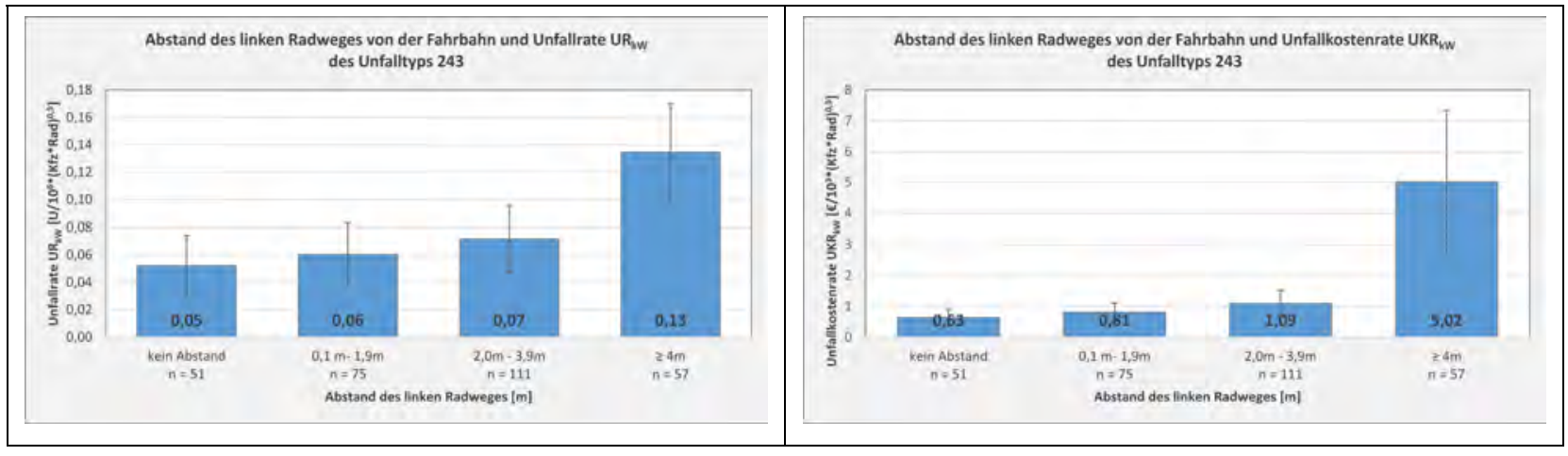

Abbildung 49: Abstand des Radweges vom Kreisrand und Unfalltyp 243

\subsection{Aufpflasterung der Furten}

An zwei Kreisverkehren des Untersuchungskollektivs sind die Querungsstellen baulich besonders gestaltet. Am Kreisverkehr in Nettetal sind die Querungsstellen gepflastert. Am Kreisverkehr in Ahaus werden die Querungsstellen baulich auf das Niveau der Rad- und Gehwege angehoben.

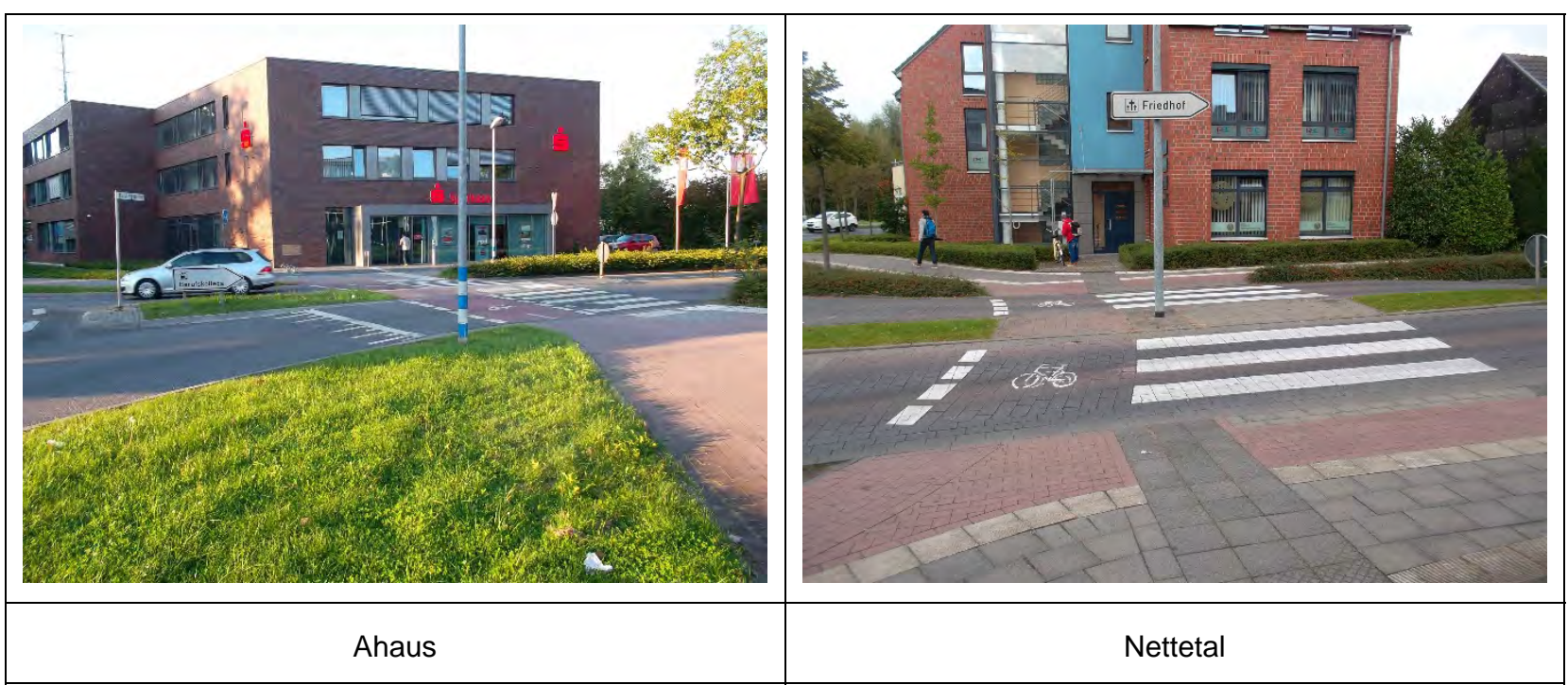

Abbildung 50: Aufpflasterung der Querungsstellen

Die folgenden Grafiken zeigen, dass keine signifikanten Unterschiede erkennbar sind. Dabei ist zu berücksichtigen, dass die Stichprobengröße nur sehr gering ist. Darüber hinaus sind die Unfalldaten für Ahaus und für Nettetal sehr unterschiedlich zu bewerten. Der Kreisverkehr in Nettetal weist insgesamt ein unterdurchschnittliches Unfallgeschehen mit Radfahrern auf, während der Kreisverkehr in Ahaus ein überdurchschnittliches Unfallgeschehen mit Radfahrern aufweist.

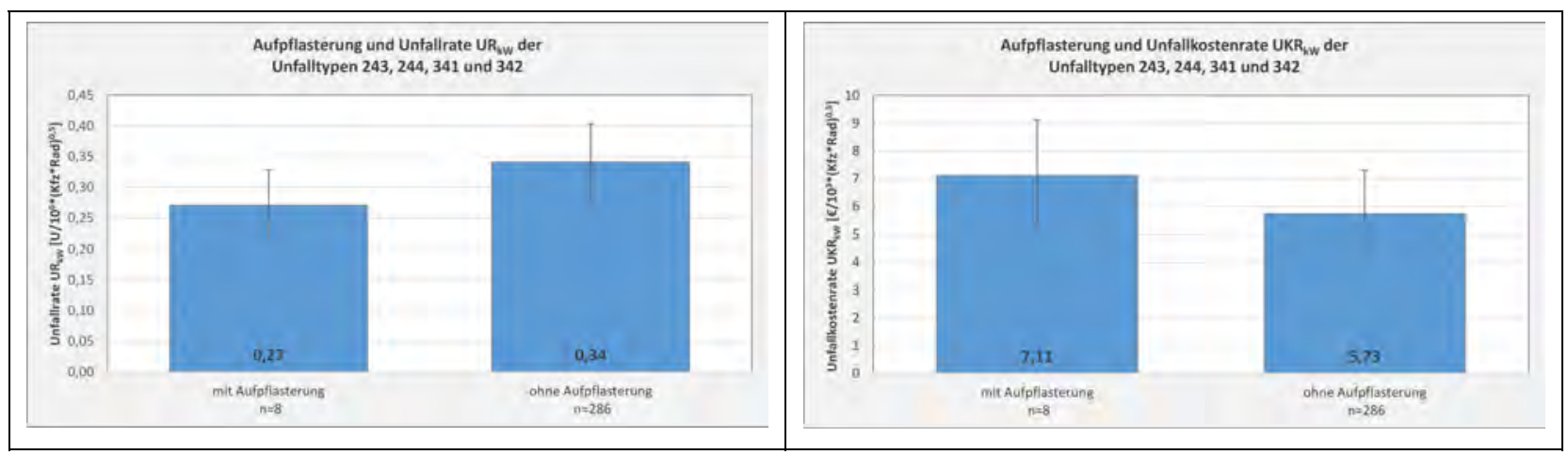

Abbildung 51: Aufpflasterung der Querungsstellen 


\subsection{Wartelinien vor den Furten}

An zwölf Kreisverkehrsarmen des Untersuchungskollektivs sind in den Ausfahrten Wartelinien vor den Querungsstellen markiert.

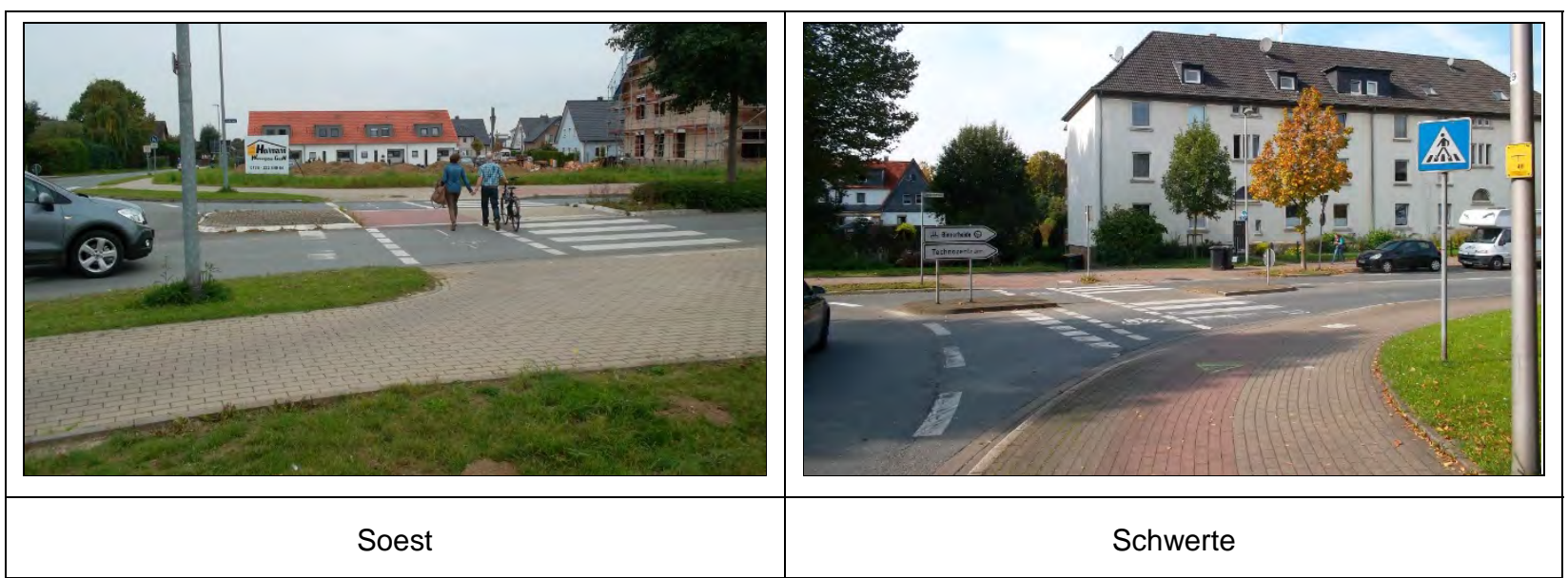

Abbildung 52: Wartelinien vor den Querungsstellen

Bei der Unfallauswertung wurden nur die Unfalltypen 243 (Kraftfahrer biegt aus, Radfahrer kommt von rechts) und 244 (Kraftfahrer biegt aus, Radfahrer kommt von links) berücksichtigt. Bezogen auf die Unfallschwere besteht kein Zusammenhang zwischen der Wartelinie und den Unfallkenngrößen. Die Unfallkostenraten sind nahezu identisch. Die Unfallraten zeigen bei den Ausfahrten ohne Wartelinien günstigere Werte.

Bei der Bewertung dieses Zusammenhangs ist allerdings zu berücksichtigen, dass insbesondere unfallauffällige Kreisverkehre möglicherweise nachträglich mit Wartelinien ausgestattet werden. Wartelinien sind daher nicht zwangsläufig die Ursache für ein höheres Unfallrisiko. Der Erfolg als Maßnahme zur Reduzierung der Unfälle mit Radfahrern lässt sich anhand des geringen Teilkollektivs nicht nachweisen.
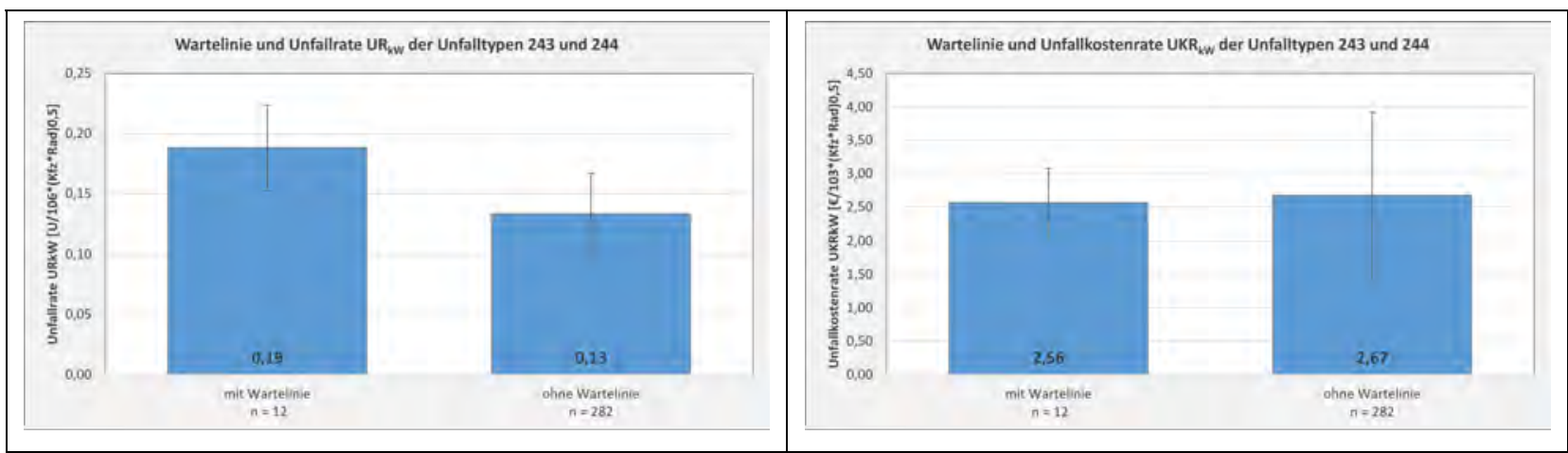

Abbildung 53: Wartelinien vor den Querungsstellen und Unfalltypen 243 und 244 


\subsection{Standort der Verkehrszeichen}

An Kreisverkehren werden in die Zufahrten die Zeichen 205 und 215 StVO angeordnet. Bei Radwegefurten werden die Verkehrszeichen im Allgemeinen vor der Querungsstelle angeordnet. Im vorliegenden Untersuchungskollektiv waren an 260 Kreisverkehrszufahrten die Verkehrszeichen vor der Querungsstelle und an 34 Kreisverkehrszufahrten hinter der Querungsstelle angeordnet.

Bei der Unfallauswertung wurden nur die Unfalltypen 341 (Kraftfahrer biegt ein, Radfahrer kommt von links) und 342 (Kraftfahrer biegt ein, Radfahrer kommt von rechts) berücksichtigt. Dabei zeigt sich, dass die Kreisverkehrszufahrten mit Anordnung der Verkehrszeichen vor der Querungsstelle ungünstigere Unfallkenngrößen aufweisen. Die Ergebnisse sind nicht erklärbar, so dass weitere Untersuchungen nötig sind.

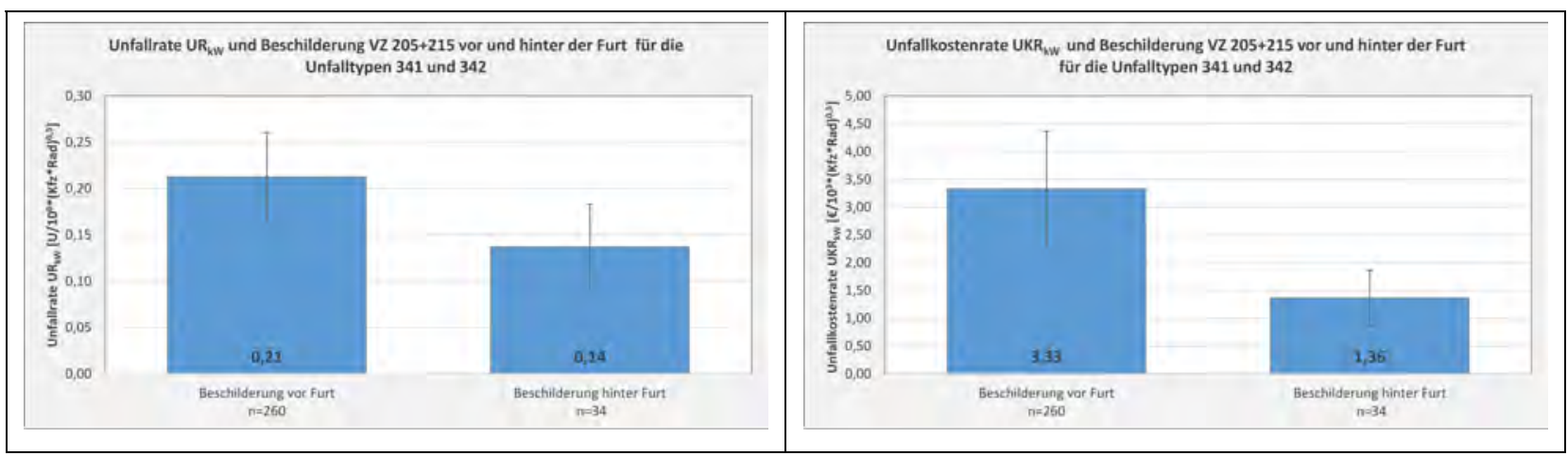

Abbildung 54: Beschilderung in den Zufahrten und Unfallgeschehen

\subsection{Beschilderung und Zweirichtungsverkehr}

Bei Furten im Zweirichtungsverkehr werden in den Zufahrten in der Regel Zusatzzeichen angebracht, die die sich nähernden Kraftfahrer auf den Zweirichtungsverkehr hinweisen. An lediglich 12 von 87 Kreisverkehrsarmen erfolgte kein Hinweis mittels Zusatzzeichen. Die folgende Abbildung zeigt, dass an den 12 Kreisverkehrsarmen keine Unfälle des Typs 341 (Kraftfahrer biegt ein, Radfahrer kommt von links) und 342 (Kraftfahrer biegt ein, Radfahrer kommt von rechts) auftraten. Ein Zusammenhang zwischen Zusatzzeichen in der Zufahrt und Unfallgeschehen ist anhand des Datenkollektivs nicht erkennbar.

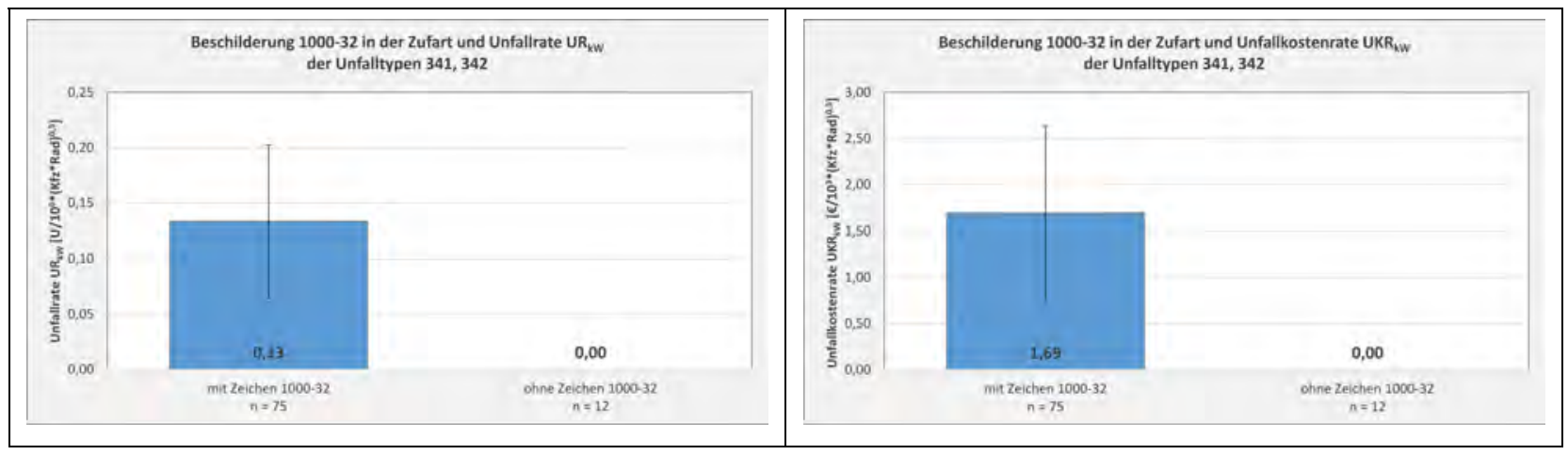

Abbildung 55: Beschilderung in den Zufahrten und Unfallgeschehen 
In den Ausfahrten sind lediglich an 6 von 87 Kreisverkehrsarmen Zusatzzeichen mit Hinweis auf den Zweirichtungsverkehr angebracht. Während bezogen auf die Unfallrate keine Unterschiede erkennbar sind, zeigt sich bei den Kreisverkehrsarmen mit Zusatzzeichen eine deutlich höhere Unfallkostenrate. Bei der Bewertung dieses Zusammenhangs ist allerdings zu berücksichtigen, dass eher unfallauffällige Kreisverkehre nachträglich mit Zusatzzeichen ausgestattet werden. Die Beschilderung ist daher aller Voraussicht nach nicht die Ursache für ein höheres Unfallrisiko. Zudem ist die Fallzahl mit nur 6 Kreisverkehrsarmen sehr gering. Als Maßnahme zur Reduzierung der Unfälle mit Radfahrern erscheint diese Maßnahme jedoch eher ungeeignet.

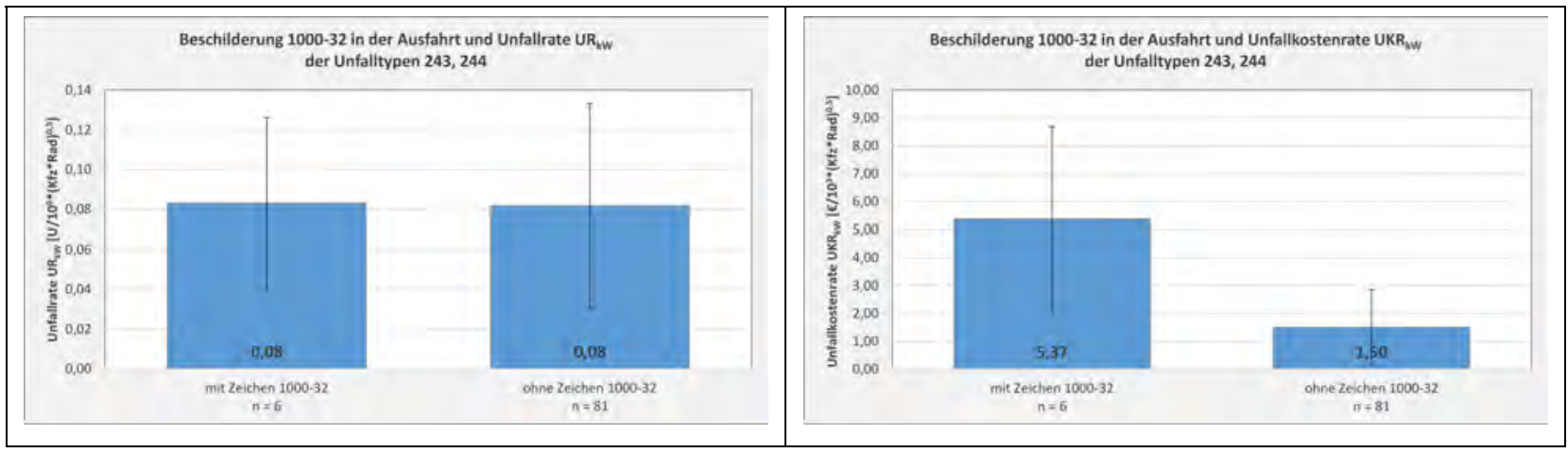

Abbildung 56: Beschilderung in den Ausfahrten und Unfallgeschehen 


\section{$9 \quad$ Multivariate Analyse}

\section{$9.1 \quad$ Methodik}

Um die Zusammenhänge der verschiedenen Variablen möglichst umfassend beschreiben zu können, wurden für die Daten zwei verschiedene multivariate Verfahren angewendet. Zum einen wurden die Daten mithilfe der multivariaten linearen Regression auf lineare Zusammenhänge untersucht und zum anderen wurde mithilfe eines Clusterverfahrens nach Strukturen zwischen den einzelnen Variablen gesucht.

Bei der multivariaten linearen Regression wird davon ausgegangen, dass die Daten aus einer abhängigen und mehreren unabhängigen Variablen bestehen. Insbesondere wird angenommen, dass die abhängige Variable linear von den unabhängigen Variablen und einem zusätzlichen Fehlerterm abhängt. Formal bedeutet dies, dass die abhängige Variable $y_{i}$ und die unabhängigen Variablen $x_{i 1}, \ldots, x_{i p}$ den folgenden Zusammenhang erfüllen:

$$
y_{i}=\beta_{1} x_{i 1}+\cdots+\beta_{p} x_{i p}+\varepsilon_{i},
$$

wobei $\beta_{1}, \ldots, \beta_{p}$ unbekannte Regressionskoeffizienten und $\varepsilon_{i}$ einen unbekannten Fehlerterm bezeichnen. Dabei haben die Fehlerterme der verschiedenen Beobachtungen den Erwartungswert 0 und sind zudem voneinander unabhängig. Ziel der multivariaten linearen Regressionsanalyse ist es die Regressionskoeffizienten zu schätzen und gleichzeitig zu untersuchen, welche der Koeffizienten überhaupt einen signifikanten Einfluss auf die abhängige Variable haben.

Für die Schätzung der Regressionskoeffizienten werden nach der Kleinsten-Quadrate-Methode diejenigen Werte für $\beta_{1}, \ldots, \beta_{p}$ gewählt, die die Quadratsumme der Residuen, also die Summe

$$
\sum_{i=1}^{n}\left(y_{i}-\beta_{1} x_{i 1}+\cdots+\beta_{p} x_{i p}\right)^{2},
$$

minimieren. Hierbei bezeichnet $\left(y_{i}, x_{i 1}, \ldots, x_{i p}\right)$ die $i$-te Beobachtung der Daten. Die Entscheidung, welche der Koeffizienten überhaupt einen signifikanten Einfluss haben, kann mithilfe eines t-Tests untersucht werden. Dieser testet für alle unabhängigen Variablen die Hypothese $\beta_{j}=0$, d.h., dass die $j$ te unabhängige Variable keinen Einfluss auf die abhängige Variable habe, gegen die Alternative $\beta_{j} \neq 0$.

Wurden signifikante Zusammenhänge in den Daten festgestellt, so gibt anschließend der geschätzte Wert der signifikanten Regressionskoeffizienten Aufschluss über die die Stärke des Zusammenhanges zwischen der entsprechenden unabhängigen und abhängigen Variablen und ob es sich um einen positiven oder negative Einfluss handelt.

Einen Maß für die Qualität der Erklärung der abhängigen Variablen durch die betrachteten Einflussgrößen ist das Bestimmtheitsmaß $R^{2}$. Je näher $R^{2}$ beim optimalen Wert von 1 liegt, desto größer ist der durch das lineare Modell erklärte Teil der Variabilität in der abhängigen Variable durch die jeweils betrachteten Einflussgrößen. Allerdings muss dies nicht bedeuten, dass ein deutlich näher bei 0 liegender Wert von $R^{2}$ auf ungeeignete (oder zu wenige) Einflussgrößen hinweist. Vielmehr beruhen die Daten für die einzelnen Kreisverkehrsarme auf jeweils wenigen Ereignissen, so dass die Varianzen groß sind. 
Für die vorliegen Daten wurden verschiedene lineare Modelle angepasst, bei denen jeweils eine der Unfallkenngrößen als abhängige Variable gewählt wurde. Als unabhängige wurden 11 Größen gewählt, die den Aufbau der Kreisverkehrsarme beschreiben. Dazu gehören sowohl kontinuierliche Variablen (Abstand des Radweges links und rechts, Verzweigungspunkt links und rechts, Absetzung des Radweges, Breite des Innenringes, Breite der Kreisinsel und Außendurchmesser) als auch kategorielle Werte (Kunst, Zweirichtungsverkehr, Furteinfärbung, Piktogramme mit und ohne Fahrtrichtung). Die Berechnung der Schätzer und die Tests auf Signifikanz der einzelnen unabhängigen Variablen wurden in der Auswertung mit der in R implementierten Funktion Im durchgeführt.

Als zweites Verfahren wurde das k-means Clusterverfahren eingesetzt, um die Daten auf Strukturen zwischen den verschiedenen Variablen zu untersuchen. Das Verfahren teilt multivariate Beobachtungen $x_{1}, \ldots, x_{n}$ dabei so in Gruppen $G_{1}, \ldots G_{k}$ ein, dass die Summe der Abstände zwischen den Datenpunkten und dem zur Gruppe gehörigen Mittelwert $m_{j}$ möglichst minimal wird. Für die vorliegenden Daten bedeutet dies, dass die verschiedenen Kreisverkehrsarme in Gruppen eingeteilt werden, deren Beobachtungen sich in ihren Unfallkenngrößen und ihrem Aufbau ähneln. Formal wird dabei, für eine zuvor gewählte Anzahl von Gruppen $k$, die Summe

$$
S=\sum_{j=1}^{k} \sum_{x_{i} \in G_{j}}\left\|x_{i}-m_{j}\right\|^{2}
$$

minimiert. Die Berechnung der Gruppenmittelwerte und der Gruppeneinteilung erfolgt mithilfe approximativer Algorithmen. In der nachfolgenden Auswertung wurde dafür die in R [21] implementierte Funktion k-means verwendet. Um zu entscheiden, wie viele Gruppen für die Aufteilung sinnvoll sind, wurde für die vorliegenden Daten die Varianz innerhalb der Gruppen, d.h. die Summe $S$, in Abhängigkeit von der Gruppenanzahl betrachtet. Wird diese für eine zusätzliche Gruppe nicht mehr „merklich“ kleiner, so ist die gewählte Gruppenanzahl ausreichend.

Beim k-means Clusterverfahren handelt es sich um ein rein deskriptives Verfahren. So können die erhaltenen Ergebnisse nicht auf Signifikanz überprüft werden, wohl aber Auffälligkeiten in den Daten erkennen und verdeutlichen. In der nachfolgenden Auswertung wurden dafür zunächst die ersten beiden Hauptkomponenten der Daten bestimmt, d.h. diejenigen Richtungen in einem durch die Beobachtungsgrößen aufgespannten Koordinatensystem, entlang derer die Beobachtungen die größte Variabilität zeigen. Eine Grafik der Daten dargestellt durch die ersten beiden Hauptkomponenten, in der zudem die Einteilung in die Gruppen kenntlich gemacht wird, kann einen ersten Einblick in den Unterschied der verschiedenen Gruppen geben. Eine entsprechende Grafik wurde in der Auswertung mit der in $\mathrm{R}$ implementierten Funktion clusplot erstellt. Des Weiteren wurden die Beobachtungen nach Gruppen getrennt in Boxplots dargestellt, um einen Einblick in die genaue Zusammensetzung der Gruppen zu erhalten. 


\subsection{Korrelationen}

\section{Furteinfärbung}

Bei der Untersuchung der Korrelationen ausgesuchter Größen zeigt sich, dass die Korrelation zwischen der Furteinfärbung und Unfallkosten, Unfalldichte, Unfallrate bzw. Unfallkostenrate signifikant von 0 verschieden ist. Interessant ist dabei, dass alle vier Korrelationen positiv sind, also dass bei vorhandener Furteinfärbung Unfallkosten, Unfalldichte, Unfallrate bzw. Unfallkostenrate größere Werte aufweisen.

Die folgenden Abbildungen zeigen die Ergebnisse der Korrelationsrechnungen. Ergänzend sind dazu die Grafiken der statistischen Analysen der Einzelmerkmale aufgeführt. Es zeigt sich, dass die Ergebnisse der statistischen Analysen der Einzelmerkmale bestätigt werden.

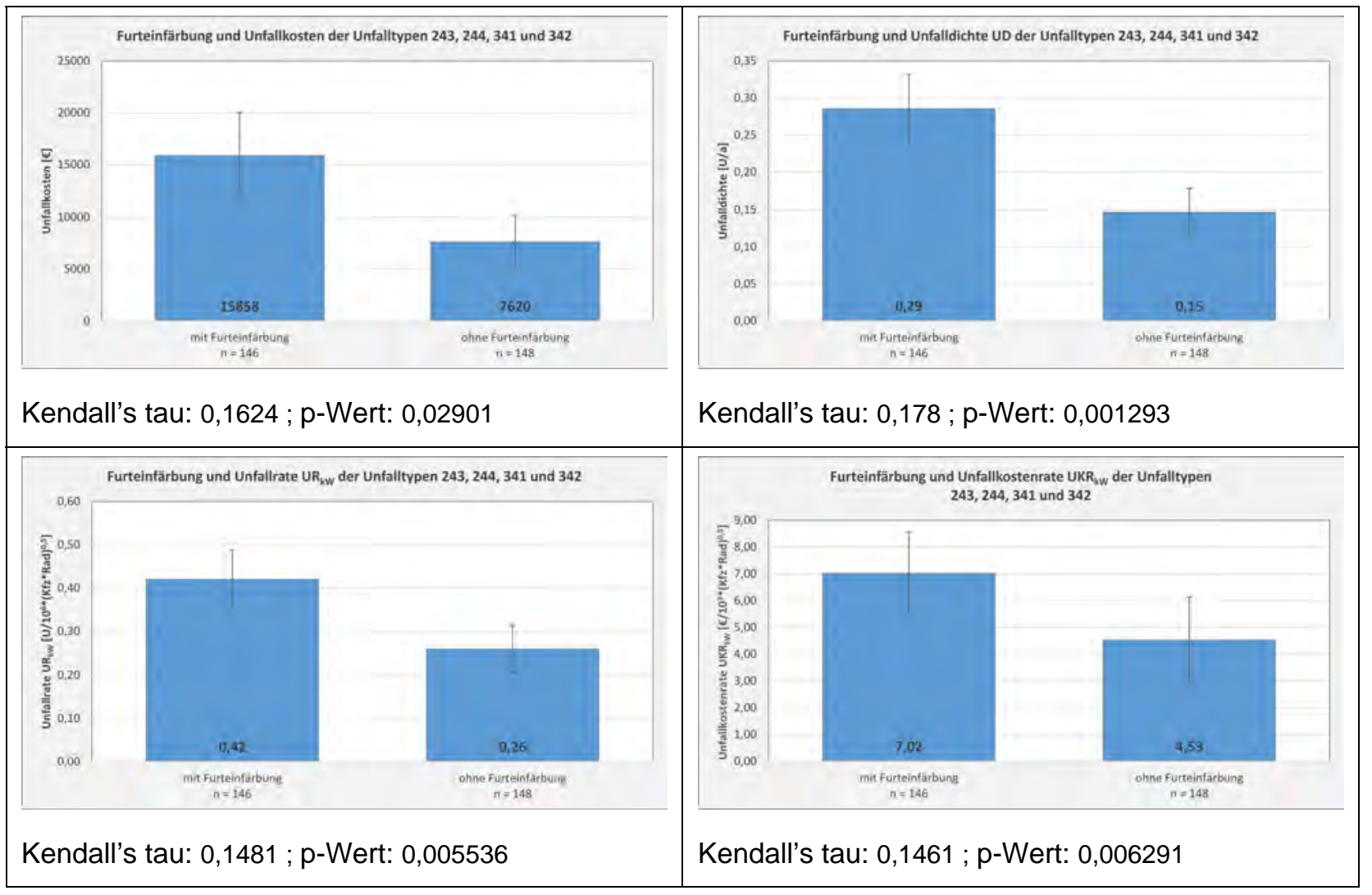

Abbildung 57: Merkmal Furteinfärbung

\section{Kunst im Kreisverkehr}

Da anzunehmen ist, dass Kunst im Kreisverkehr nur einen Einfluss auf einfahrende Autofahrer hat, wird ihr Effekt auf die entsprechenden Unfalltypen untersucht. Dabei zeigt sich, dass Kunst einen signifikanten Einfluss auf Unfallkosten und Unfallkostenrate bei Unfalltyp 341 (Kraftfahrer biegt ein, Radfahrer kommt von links) hat. Beide Größen sind bei vorhandener Kunst höher.

Die folgenden Abbildungen zeigen die Ergebnisse der Korrelationsrechnungen. Ergänzend sind dazu die Grafiken der statistischen Analysen der Einzelmerkmale aufgeführt. Es zeigt sich, dass die Ergebnisse der statistischen Analysen der Einzelmerkmale bestätigt werden. 


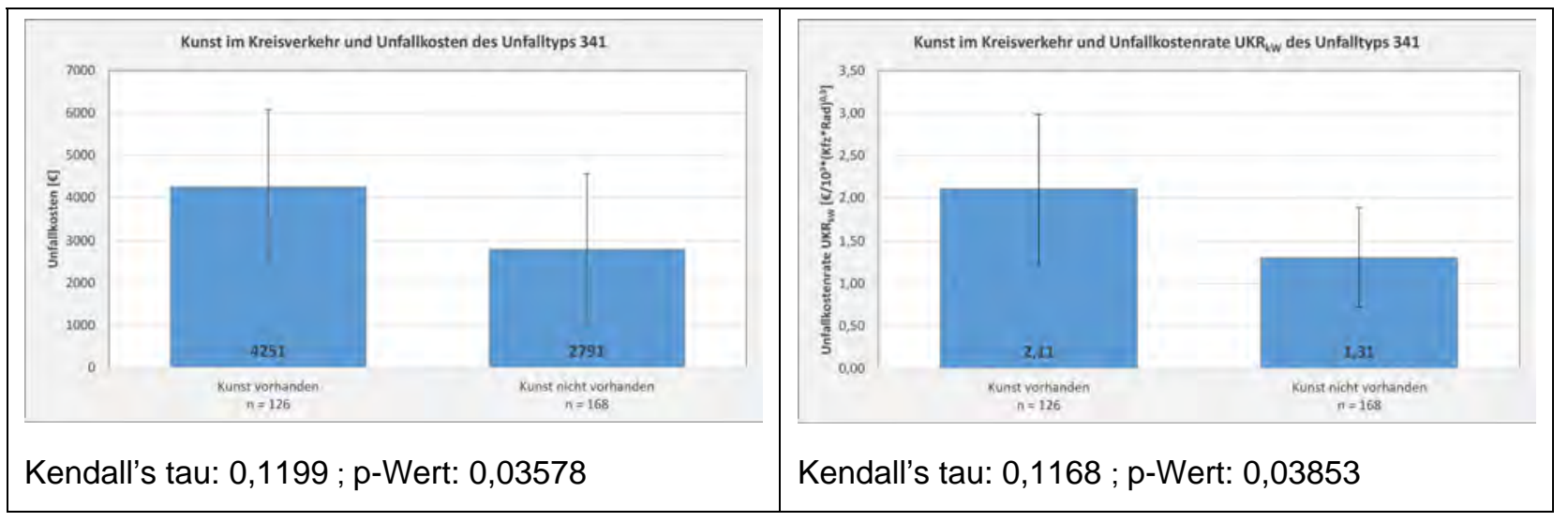

Abbildung 58: Merkmal Kunst im Kreis

\section{Lage des Verzweigungspunktes}

Der Einfluss der Verzweigungspunkte der Radfahrer auf die Unfalltypen 243 (Kraftfahrer biegt aus, Radfahrer kommt von rechts) bzw. 342 (Kraftfahrer biegt ein, Radfahrer kommt von rechts) wurde untersucht. Beim Unfalltyp 243 wurde der Verzweigungspunkt vor der Ausfahrt des Kreisverkehrs untersucht. Dabei sind alle betrachteten Korrelationen signifikant. Unfallkosten, Unfalldichte, Unfallrate und Unfallkostenrate sind bei größerem Abstand tendenziell größer.

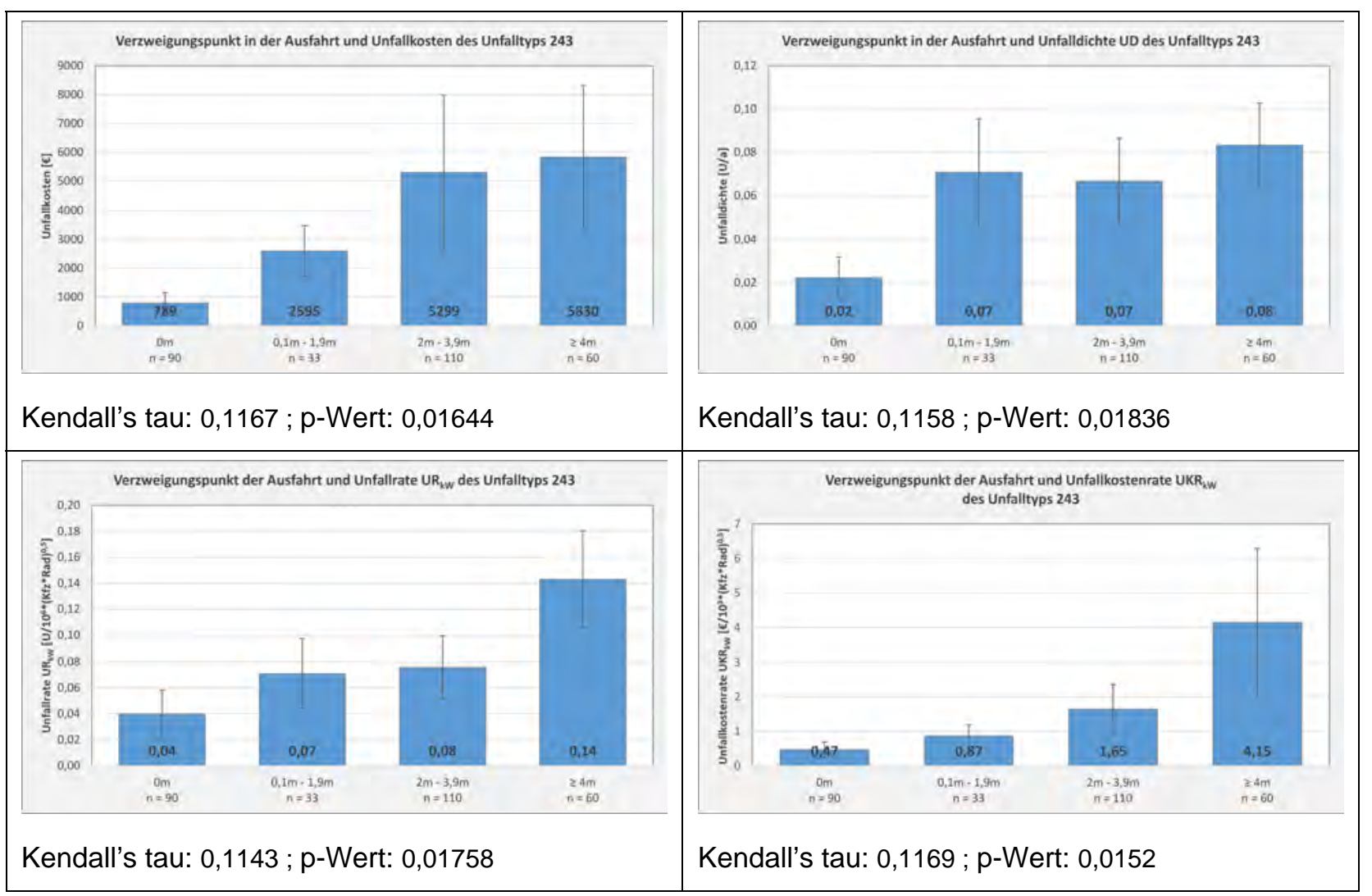

Abbildung 59: Merkmal Verzweigungspunkt vor der Ausfahrt des Kreisverkehrs

Beim Unfalltyp 342 (Kraftfahrer biegt ein, Radfahrer kommt von rechts) lässt sich kein signifikanter Zusammenhang zum Verzweigungspunkt vor der Zufahrt des Kreisverkehrs feststellen. 


\subsection{Multivariates Lineares Modell}

\section{Breite des Innenrings und Abstand des Radweges in der Ausfahrt}

Die Unfallkostenrate hängt signifikant vom Abstand des Radweges in der Ausfahrt und von der Breite des Innenringes ab. Sowohl bei größerem Abstand des Radweges als auch bei breiteren Innenringen steigt die Unfallkostenrate. Um sicherzustellen dass diese beiden Größen nicht abhängig sind und somit beide durch eine dritte Größe "zur Verfügung stehender Platz" ersetzt werden können, wurde zudem eine Korrelationsanalyse durchgeführt. Hier lässt sich keine signifikante Abhängigkeit feststellen.

Die folgende Abbildung zeigt die Auswertung nach den Einzelmerkmalen. Auch hier wird deutlich, dass die Kombination großer Abstand des Radwegs vom Kreisrand und breiter Innenring insgesamt die deutlich höchste Unfallkostenrate mit sich bringt.

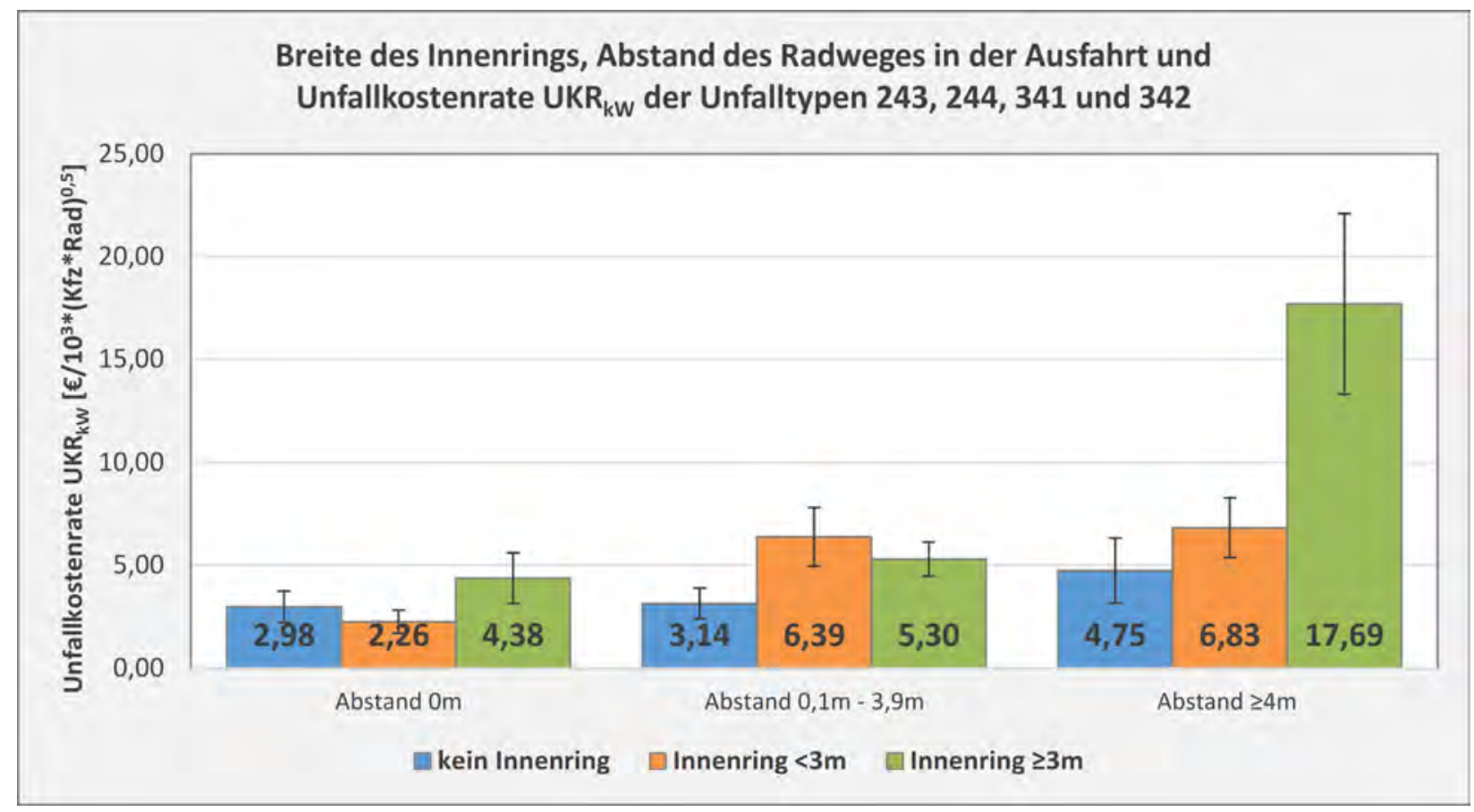

Abbildung 60: Merkmale Breite des Innenrings und Abstand des Radwegs in der Ausfahrt und alle Unfalltypen

\section{Breite des Innenrings}

Die Unfallkostenrate für Unfälle, die bei der Ausfahrt des Autos aus dem Kreisverkehr (Unfalltypen 243 (Kraftfahrer biegt aus, Radfahrer kommt von rechts) und 244 (Kraftfahrer biegt aus, Radfahrer kommt von links)) passieren, hängt signifikant von der Breite des Innenringes ab. Ist diese größer, so ist auch die Unfallkostenrate höher.

Die folgende Abbildung zeigt die Ergebnisse der statistischen Analyse des Einzelmerkmals „Breite des Innenrings“. Es zeigt sich, dass die Ergebnisse der statistischen Analysen des Einzelmerkmals bestätigt werden. 


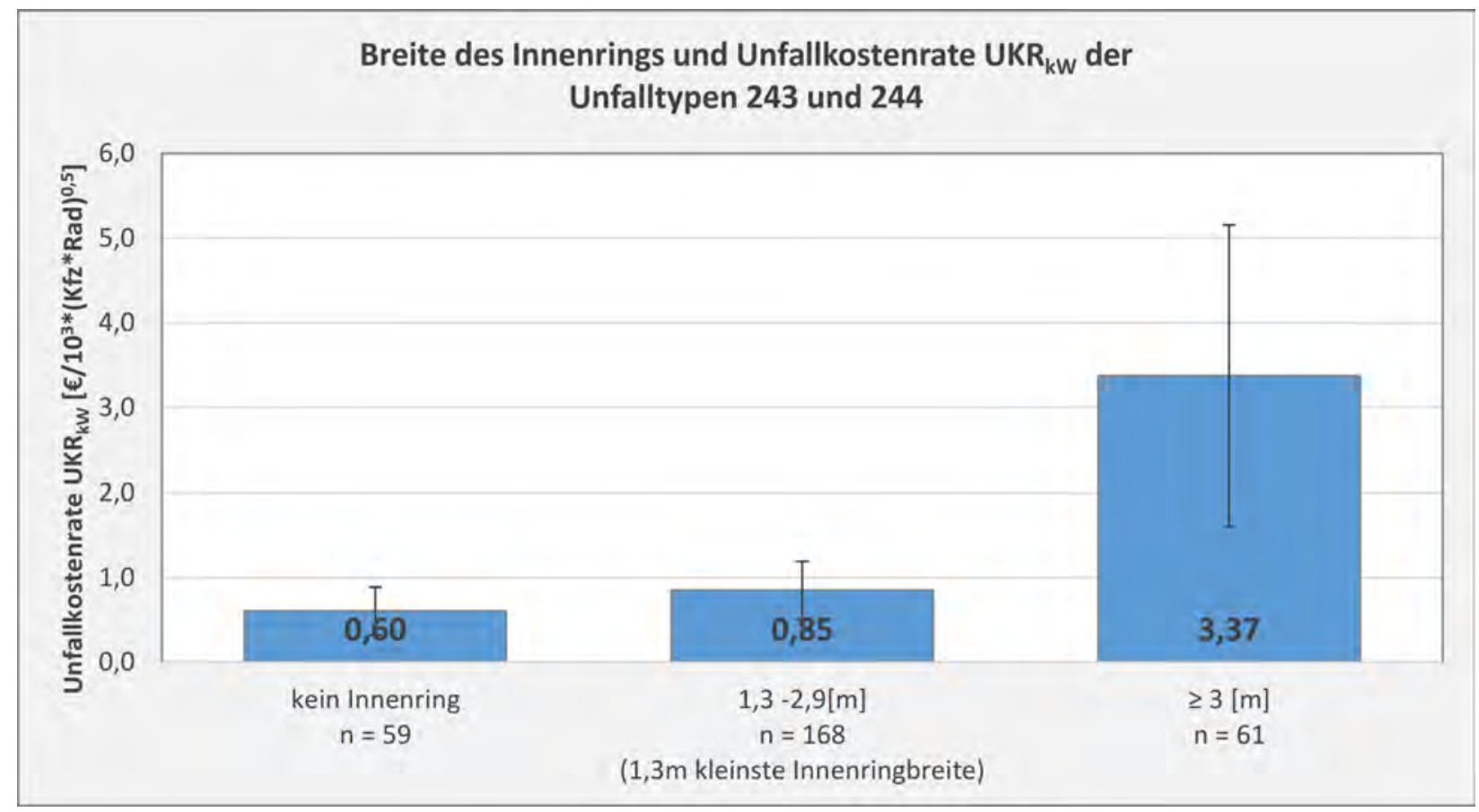

Abbildung 61: Merkmal Breite des Innenrings und Unfalltypen 243 und 244

Die Unfallkostenrate für Unfalltyp 243 (Kraftfahrer biegt aus, Radfahrer kommt von rechts) hängt signifikant von der Breite des Innenringes ab. Ist diese größer, so ist auch die Unfallkostenrate höher.

Die folgende Abbildung zeigt die Ergebnisse der statistischen Analyse des Einzelmerkmals „Breite des Innenrings". Es zeigt sich, dass die Ergebnisse der statistischen Analysen des Einzelmerkmals bestätigt werden.

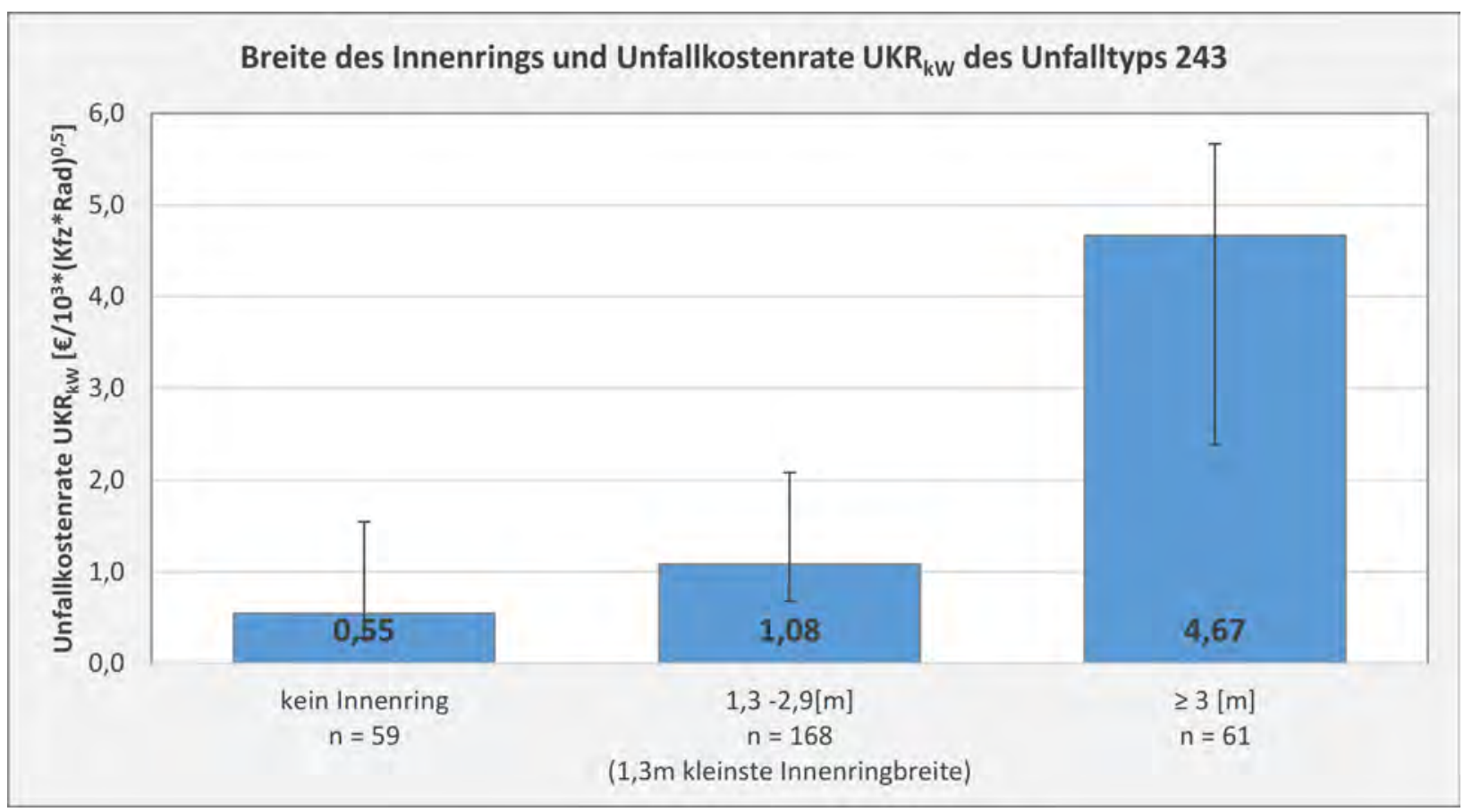

Abbildung 62: Merkmal Breite des Innenrings und Unfalltyp 243 


\section{Radfahrer im Uhrzeigersinn und Unfallkostenrate}

Die Unfallkostenrate für Unfälle mit Fahrtrichtung im Uhrzeigersinn (Unfalltypen 244 (Kraftfahrer biegt aus, Radfahrer kommt von links) und 342(Kraftfahrer biegt ein, Radfahrer kommt von rechts)) ist signifikant vom Zweirichtungsverkehr und von Piktogrammen mit Fahrtrichtung abhängig. Bei Zweirichtungskreisverkehren ist sie höher und bei Piktogrammen mit Fahrtrichtung sinkt sie. Im linearen Modell wird angedeutet, dass Zweirichtungsverkehre mit Piktogrammen mit Fahrtrichtung insgesamt eine niedrigere Unfallkostenrate haben als Kreisverkehre, die beides nicht besitzen.

Die folgende Abbildung zeigt die Ergebnisse der statistischen Analyse der Merkmale "Zweirichtungsverkehr" und „Piktogramme mit Fahrtrichtung“. Es zeigt sich, dass die Ergebnisse der statistischen Analysen der Einzelmerkmale bestätigt werden.

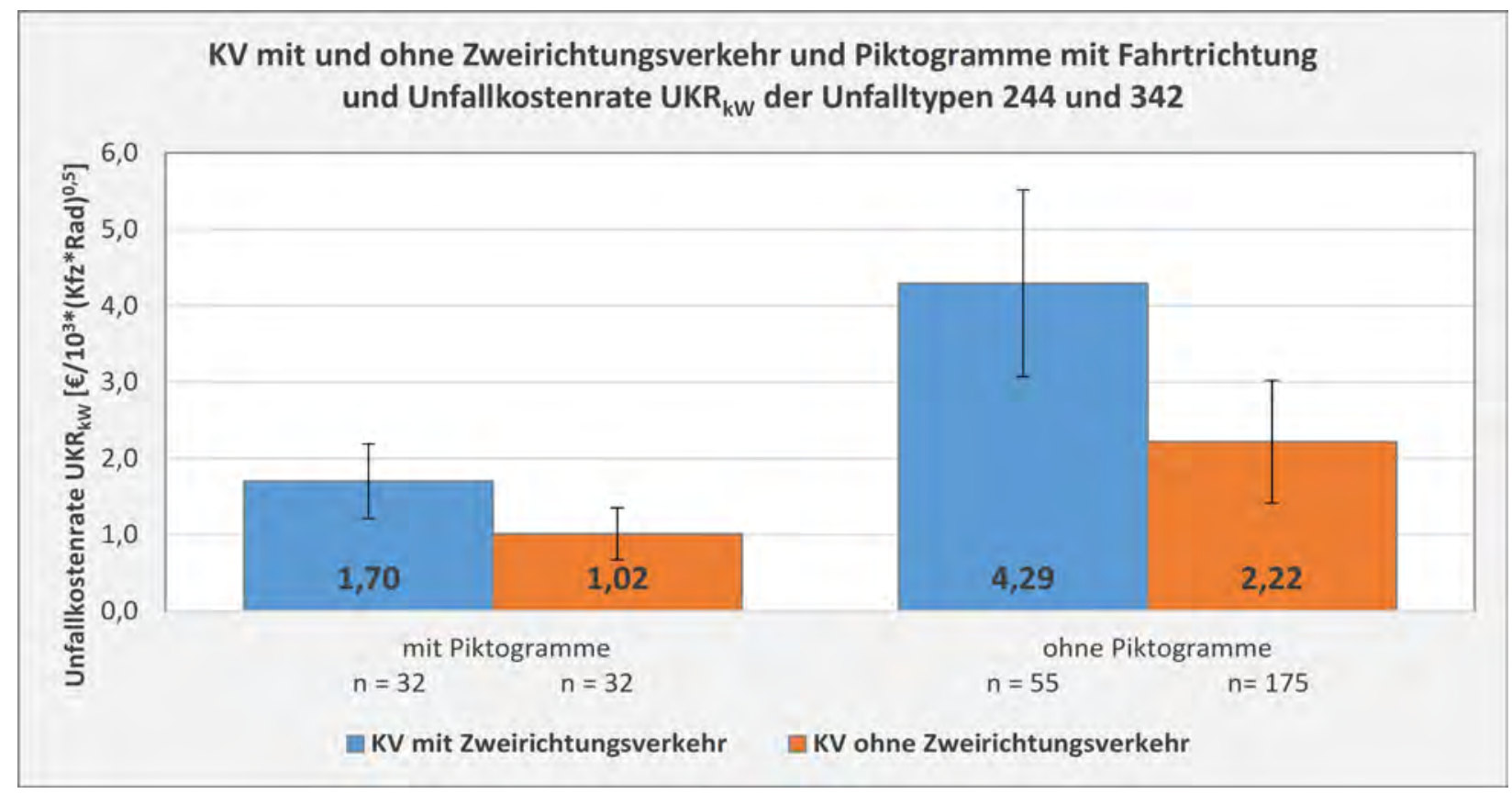

Abbildung 63: Merkmale Zweirichtungsverkehr und Piktogramme in Fahrtrichtung und Unfalltypen 244 und 342

\subsection{Multivariates Clusterverfahren}

Im Folgenden wird untersucht, ob sich die Daten in verschiedene Gruppen einteilen lassen. Dafür wird das "k-means" Clusterverfahren angewendet. Dieses Verfahren teilt die Daten in ähnliche Gruppen ein. Die Gruppenanzahl wird dabei auf 4 gesetzt, da sich auch bei mehr Gruppen die Fehlersumme innerhalb der Gruppen nicht mehr deutlich verringert. 


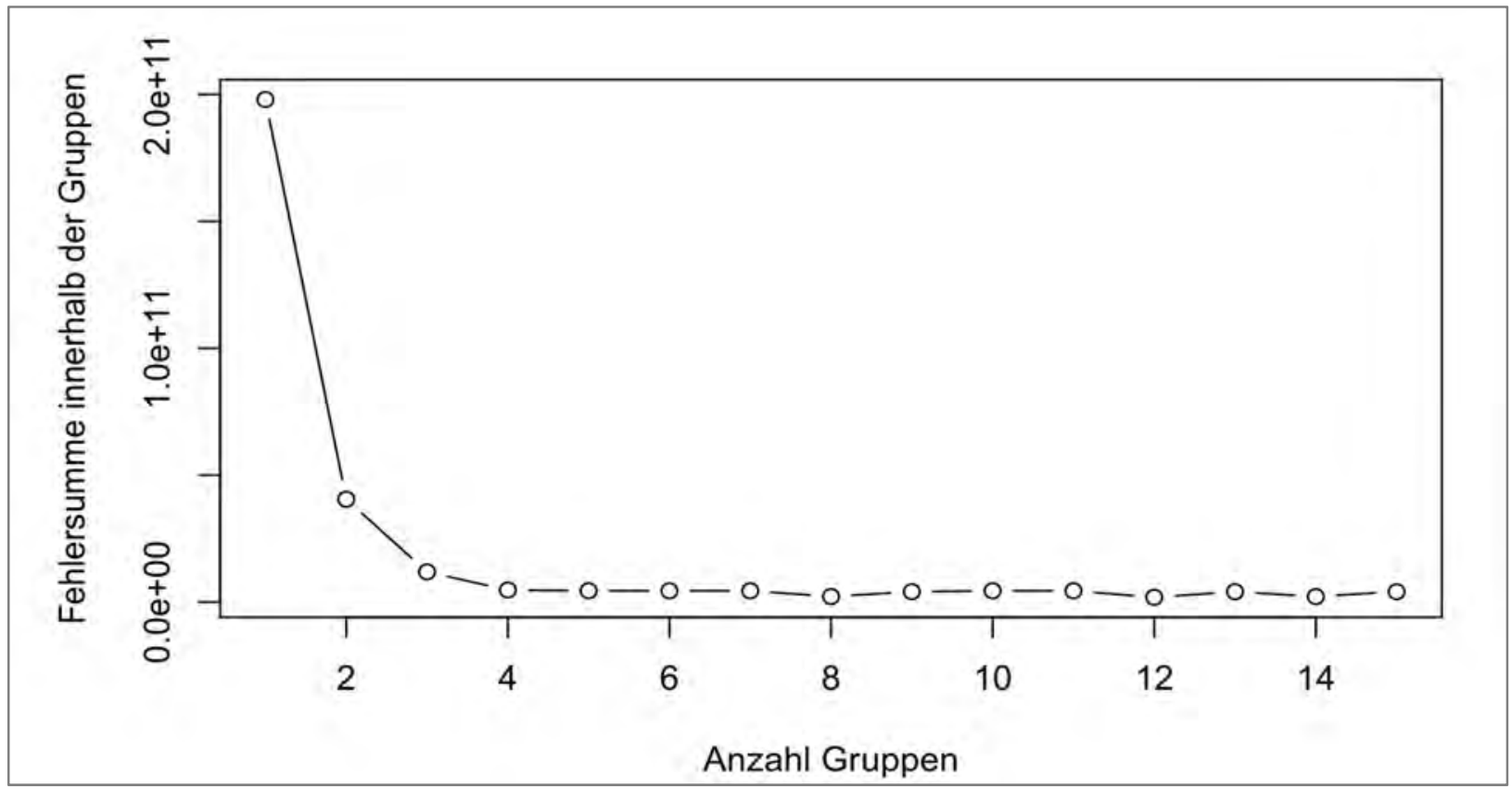

Abbildung 64: Festlegung der Anzahl der Gruppen

Um die Zusammensetzung der verschiedenen Gruppen besser zu verstehen, werden zunächst diejenigen Hauptkomponenten (Linearkombinationen aus (skalierten) Beobachtungsgrößen) bestimmt, die besonders gut zwischen den einzelnen Kreisverkehren trennen. Die folgende Grafik zeigt die Gruppeneinteilung der Daten, wenn die Daten durch die ersten beiden Hauptkomponenten dargestellt werden.

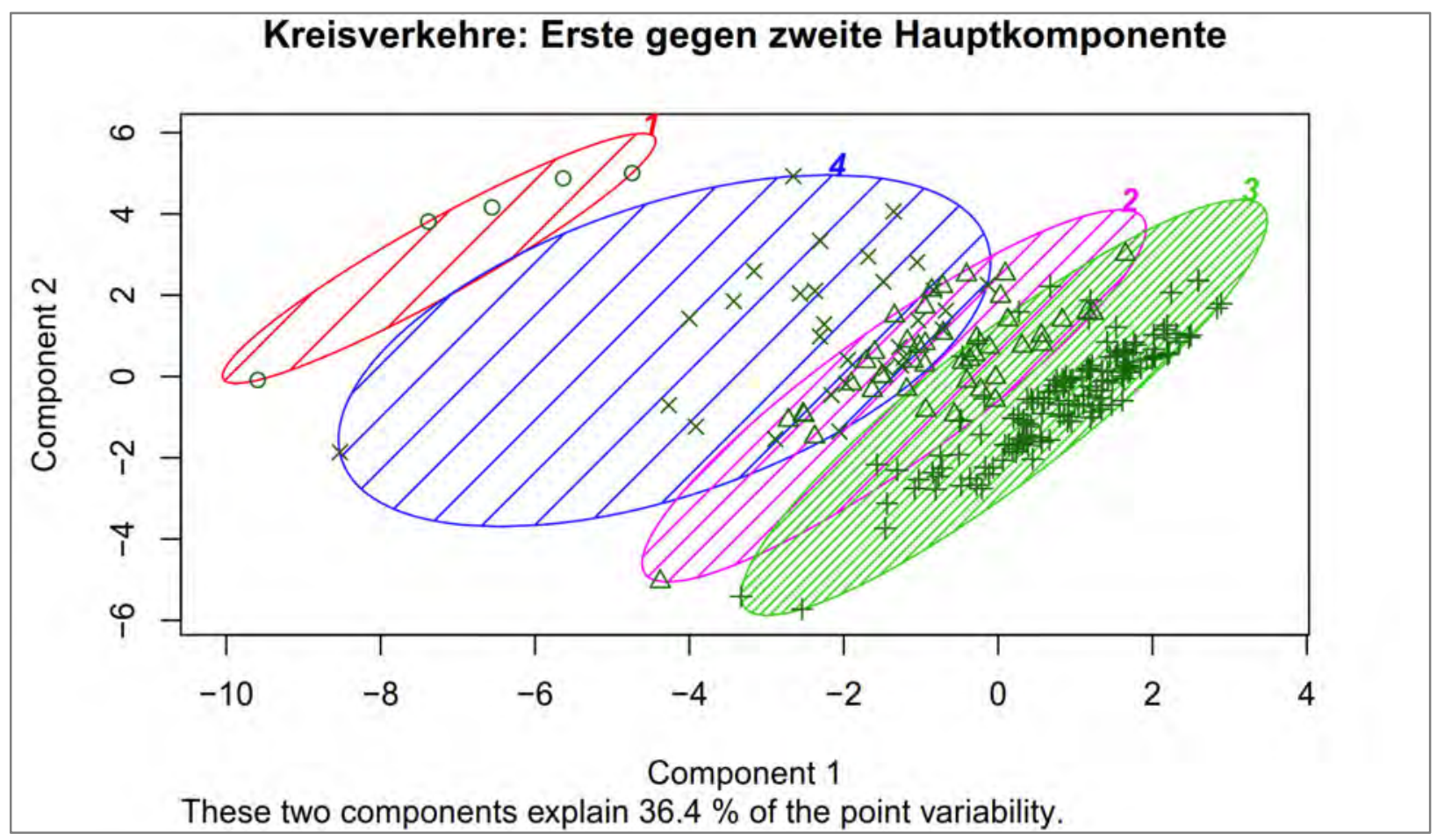

Abbildung 65: Gruppeneinteilung 
Die Unterschiede liegen vor allem in den Unfallkosten, der Unfalldichte und der Unfallkostenrate. Eine kleine Gruppe (Gruppe 1) unterscheidet sich dabei durch ihre deutlich höhere Unfallkostenrate. Zu der Gruppe 1 gehören folgende Kreisverkehrsarme:

- Ahaus, Wessumerstraße (K 17) W

- Celle, Wiesenstraße

- Delmenhorst, Seestraße

- Greven, Emsdettener Landstraße (K 53)

- Wesseling, Ahrstraße

In den folgenden Abbildungen werden für die Kreisverkehre in den einzelnen Clustern Boxplots der Unfallkosten, -dichten und -raten gezeigt. Deutlich zeigt sich, dass sich die erste Gruppe der Kreisverkehre insbesondere bei den Unfallkosten und der Unfallkostenrate deutlich hervorhebt.

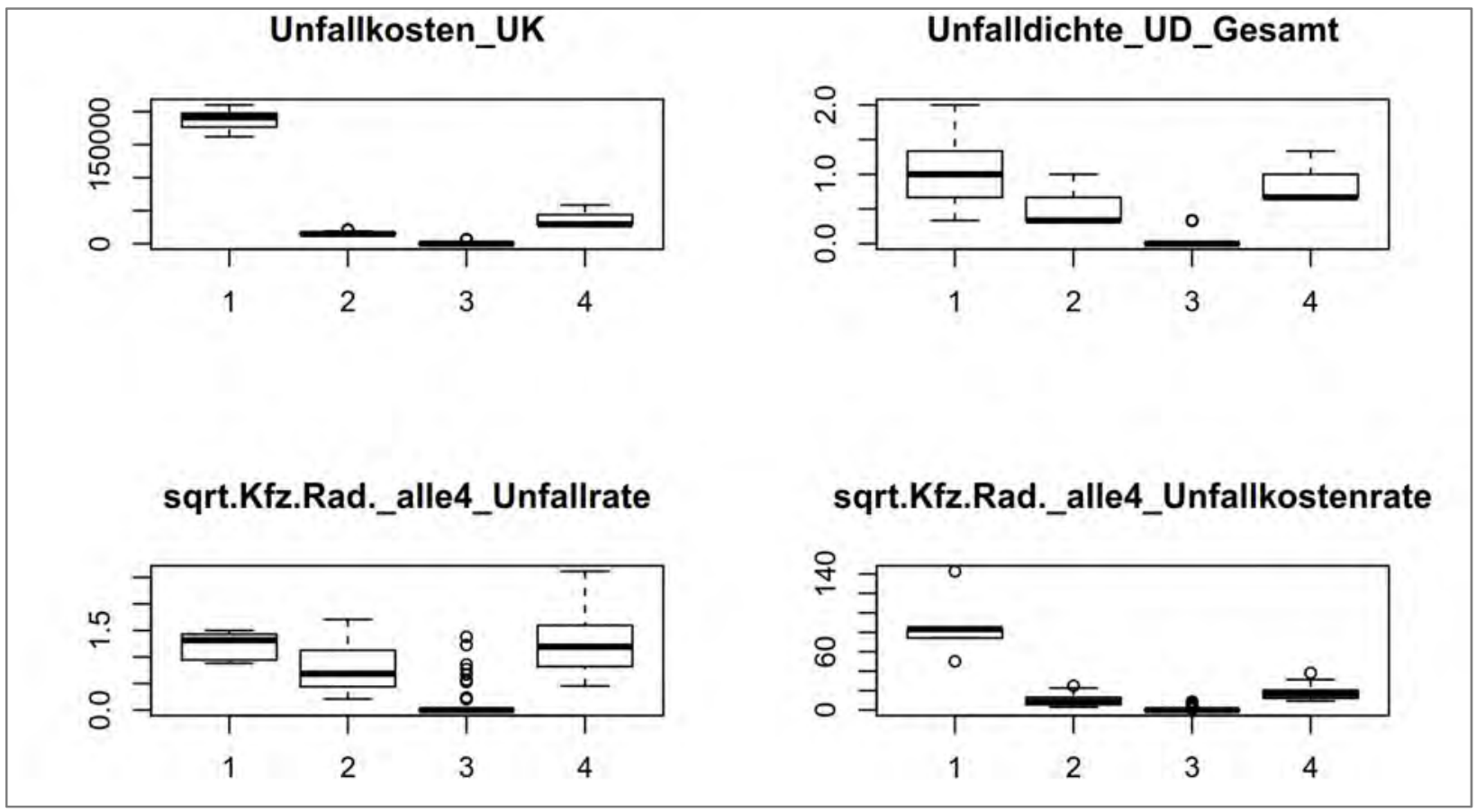

Abbildung 66: Boxplots der Unfallkenngrößen

Die auffälligen Kreisverkehre weisen insbesondere bei den Merkmalen

- Abstand des Radweges vom Kreisrand links und

- Durchmesser der Kreisinsel

Gemeinsamkeiten auf. Sie zeichnen sich durch einen im Mittel größeren Abstand des Radwegs vom Kreisrand und durch einen im Mittel geringeren Kreisinseldurchmesser aus. Die Ergebnisse der statischen Analysen der Einzelmerkmale werden bestätigt. 


\section{Vorher-Nachher-Vergleich}

Bei den beiden Kreisverkehren

- Ahaus, Adenauerring und

- Coesfeld, Dülmener Straße

wurden im Jahr 2010 Maßnahmen zur Erhöhung der Verkehrssicherheit für Radfahrer vorgenommen. Für beide Kreisverkehre lagen aus der Untersuchung aus 2012 [1] die Unfalldaten für den Vorher-Zeitraum der Jahre 2007 bis 2009 vor.

\subsection{Ahaus, Adenauerring}

Am Kreisverkehr Adenauerring in Ahaus wurden die Querungsstellen für Fußgänger und Radfahrer im Verlauf des Jahres 2010 durch Plateaus in etwa auf das Niveau der Geh- und Radwege angehoben. Zudem wurde eine Neutrassierung des Radweges im Bereich zwischen Adenauerring und Wessumer Straße West vorgenommen. Im Vorfeld der Baumaßnahme fand bereits ein Rückschnitt des kreisnahen Bewuchses statt.

Die folgende Abbildung zeigt die Ausbildung der Plateaus an den Querungsstellen:

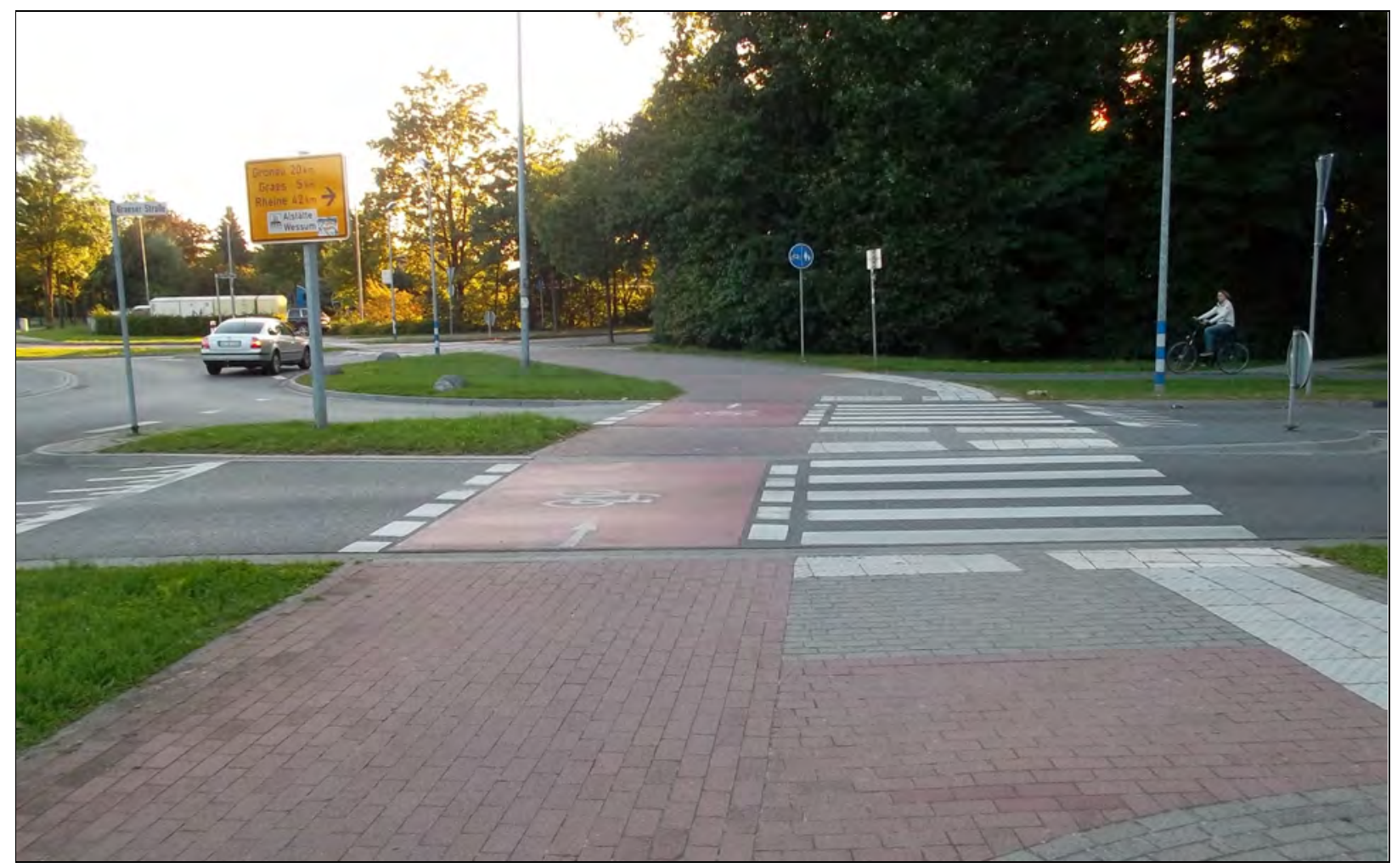

Abbildung 67: Kreisverkehr Ahaus, Adenauerring - Ausbildung der Plateaus 
Die folgende Abbildung zeigt die Unfallentwicklung in den Jahren 2007 bis 2009 vor Umsetzung der Maßnahmen sowie 2011 bis 2013 nach Umsetzung der Maßnahmen. Betrachtet wurden die Unfälle mit Radfahrerbeteiligung der Typen 243, 244, 341 und 342 in den vier Knotenpunktarmen Wessumer Straße West, Wessumer Straße Süd, Fuistungstraße und Graeser Straße. Der Knotenpunktarm Adenauerring wurde aufgrund der Besonderheit des einseitigen Zweirichtungsradwegs nicht betrachtet.

Es zeigt sich, dass insgesamt die Anzahl der Radfahrerunfälle von 15 Unfällen / 3 Jahre auf 9 Unfälle / 3 Jahre abgenommen hat. Die Anzahl der Leichtverletzten hat sich von vorher 10 auf nachher 3 reduziert. Allerdings wurde im Nachher-Zeitraum ein Radfahrer schwer verletzt. Im Jahr nach Umsetzung der Maßnahmen (2011) fand ein deutlicher Rückgang statt. Nachdem die Unfallzahlen im Jahr 2012 wieder auf das Niveau des Vorher-Zeitraums angestiegen waren, war im Jahr 2013 wiederum ein deutlicher Rückgang zu verzeichnen.

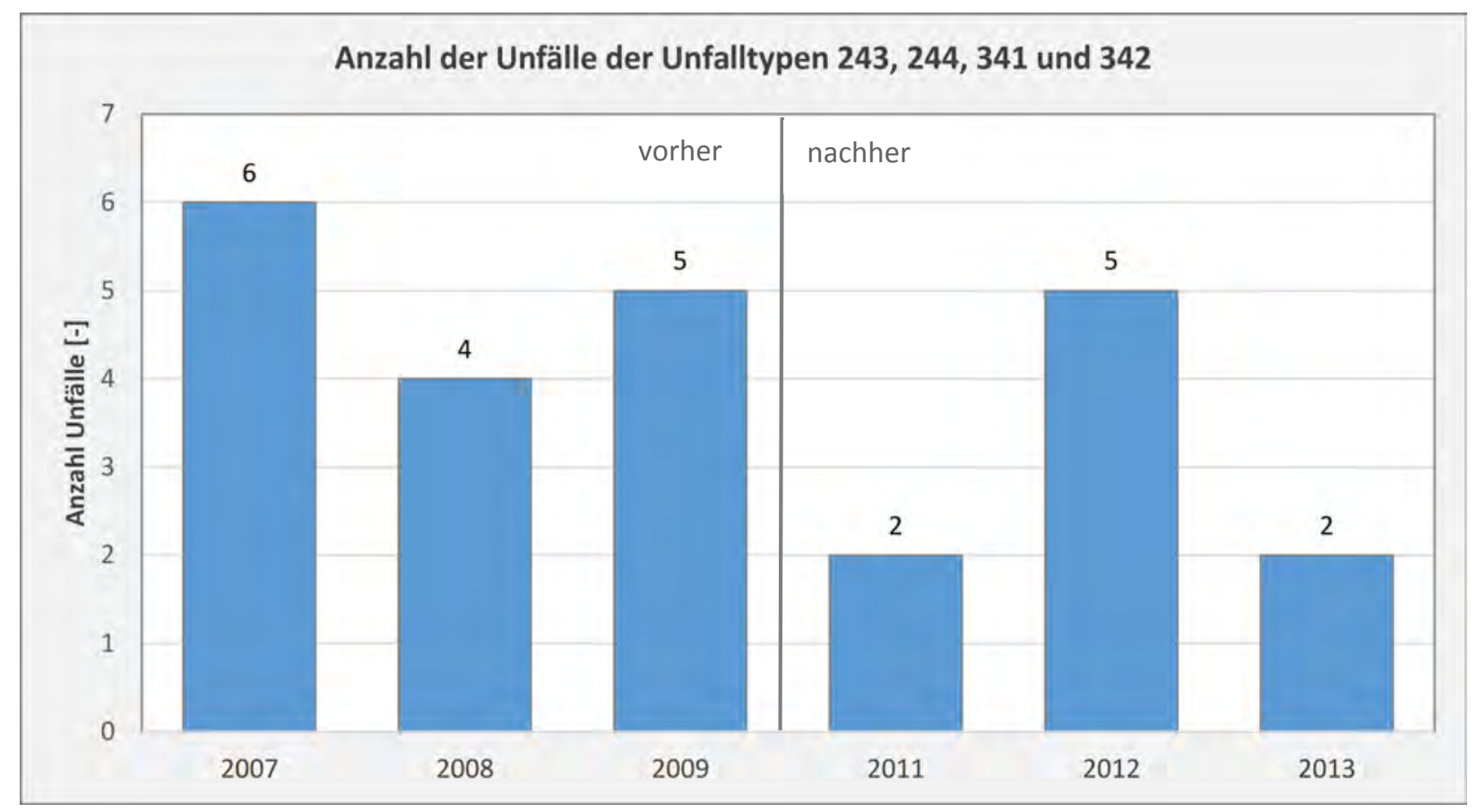

Abbildung 68: Kreisverkehr Ahaus, Adenauerring - Unfallentwicklung

Die folgenden Abbildungen zeigen die Unfallkenngrößen im Vorher-Nachher-Vergleich getrennt nach Knotenpunktarmen. Auffallend ist der Anstieg der Unfallrate und der Unfallkostenrate in der Wessumer Straße West. Aufgrund eines Schwerletzten im Nachher-Zeitraum ist ein insbesondere bei der Unfallkostenrate ein erheblicher Anstieg zu verzeichnen. In der Wessumerstraße Süd traten im NachherZeitraum keine Unfälle der relevanten Unfalltypen und damit eine deutliche Verbesserung der Unfallsituation im Vergleich zum Vorher-Zeitraum auf. In den Knotenpunktarmen Fuistingstraße und Graeser Straße ist im Nachher-Zeitraum ebenfalls eine Reduzierung des Unfallgeschehens zu verzeichnen. 


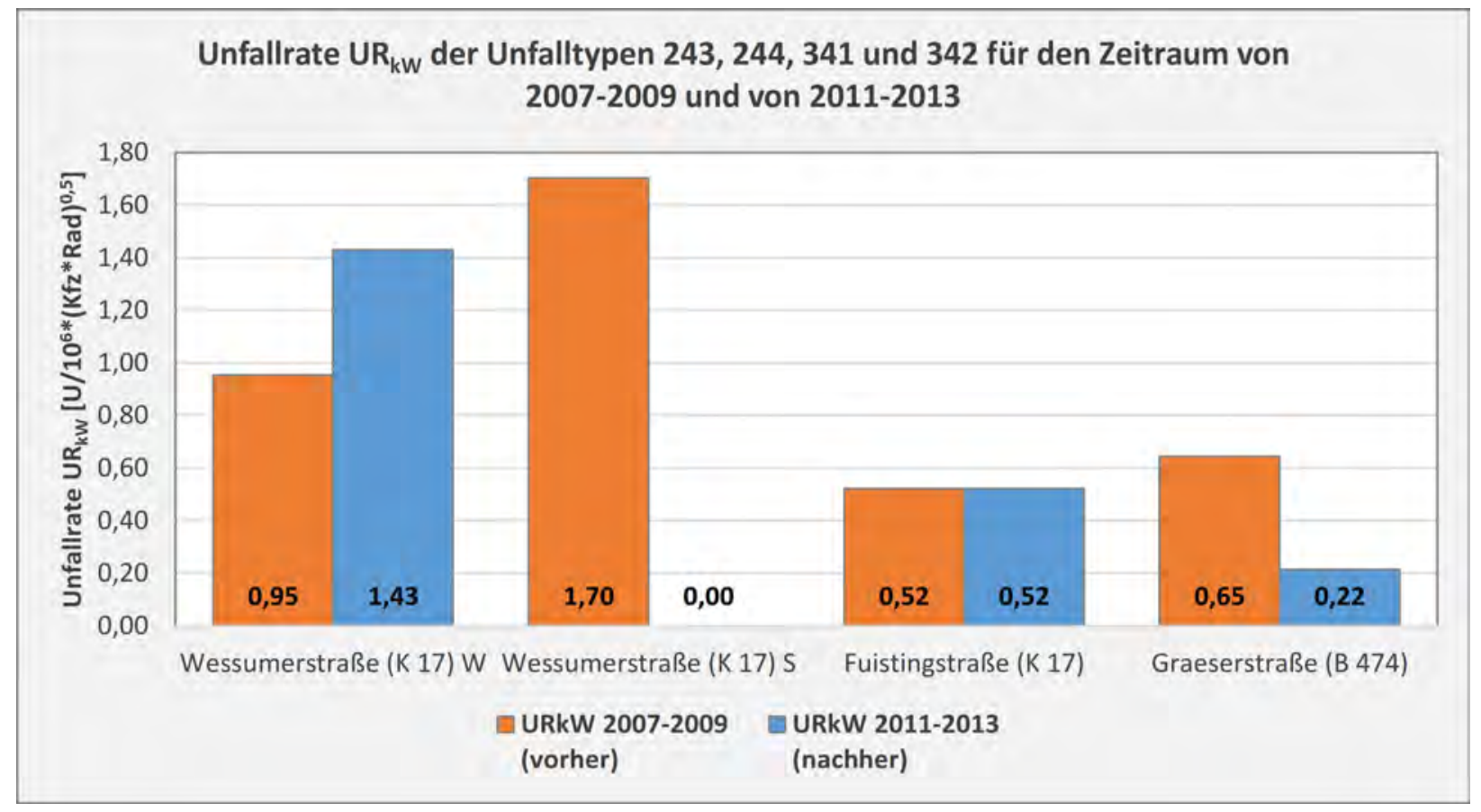

Abbildung 69: Kreisverkehr Ahaus, Adenauerring, Vorher-Nachher-Vergleich, getrennt nach Knotenpunktarmen, $\mathrm{UR}_{\mathrm{kW}}$

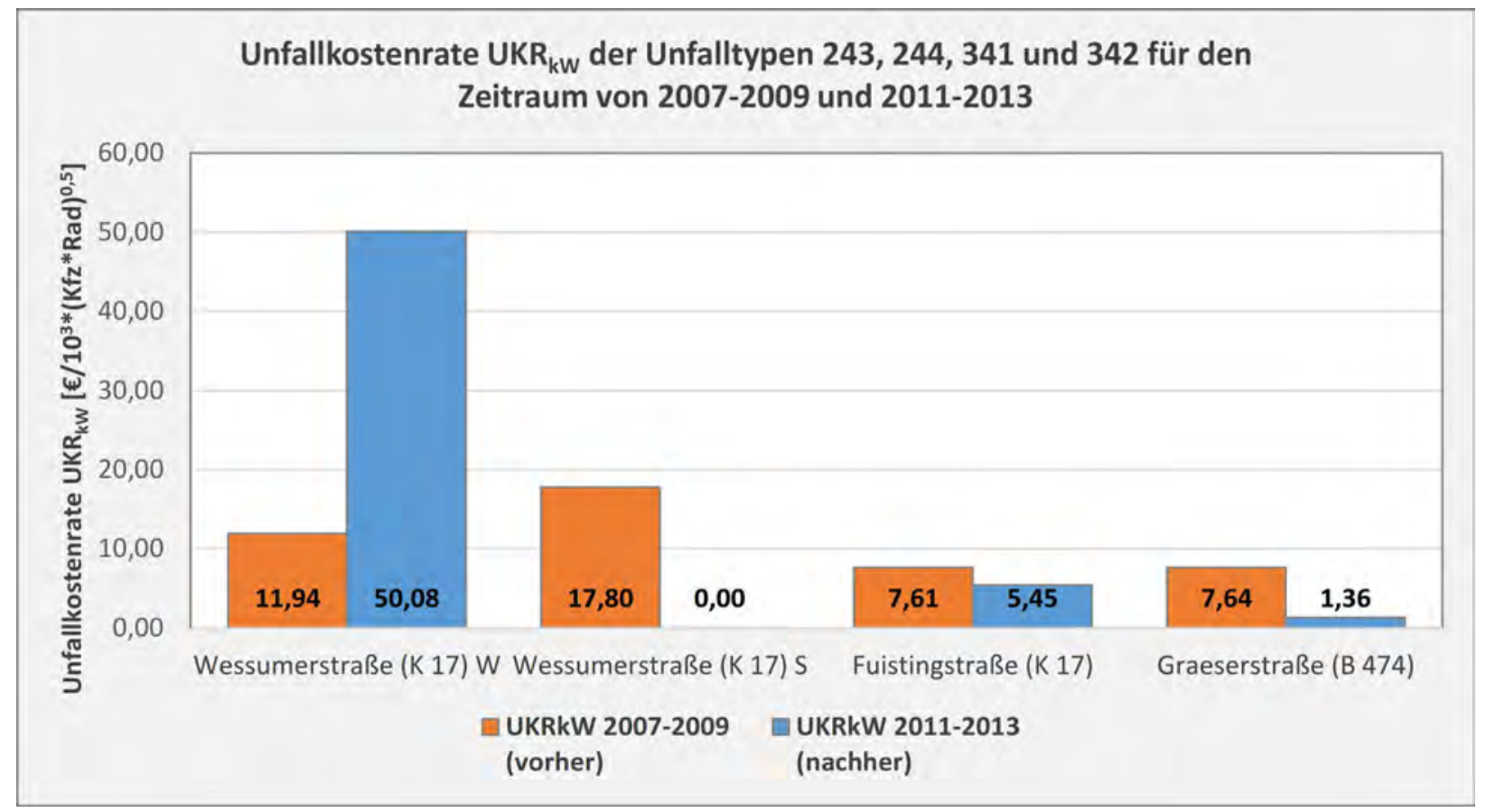

Abbildung 70: Kreisverkehr Ahaus, Adenauerring, Vorher-Nachher-Vergleich, getrennt nach Knotenpunktarmen, $\mathrm{UKR}_{\mathrm{kW}}$

Zusammenfassend sind die umgesetzten Maßnahmen als erfolgreich zu bewerten. Die Anzahl der relevanten Unfälle mit Radfahrerbeteiligung ist deutlich zurückgegangen. Die einzige Ausnahme bildet der westliche Arm der Wessumer Straße mit einer Erhöhung der Unfallzahlen. 


\subsection{Coesfeld, Dülmener Straße}

Am Kreisverkehr Dülmener Straße in Coesfeld wurden die Radfahrerfurten im Jahr 2010 rot eingefärbt. Die folgende Abbildung zeigt die Gestaltung der Furten.

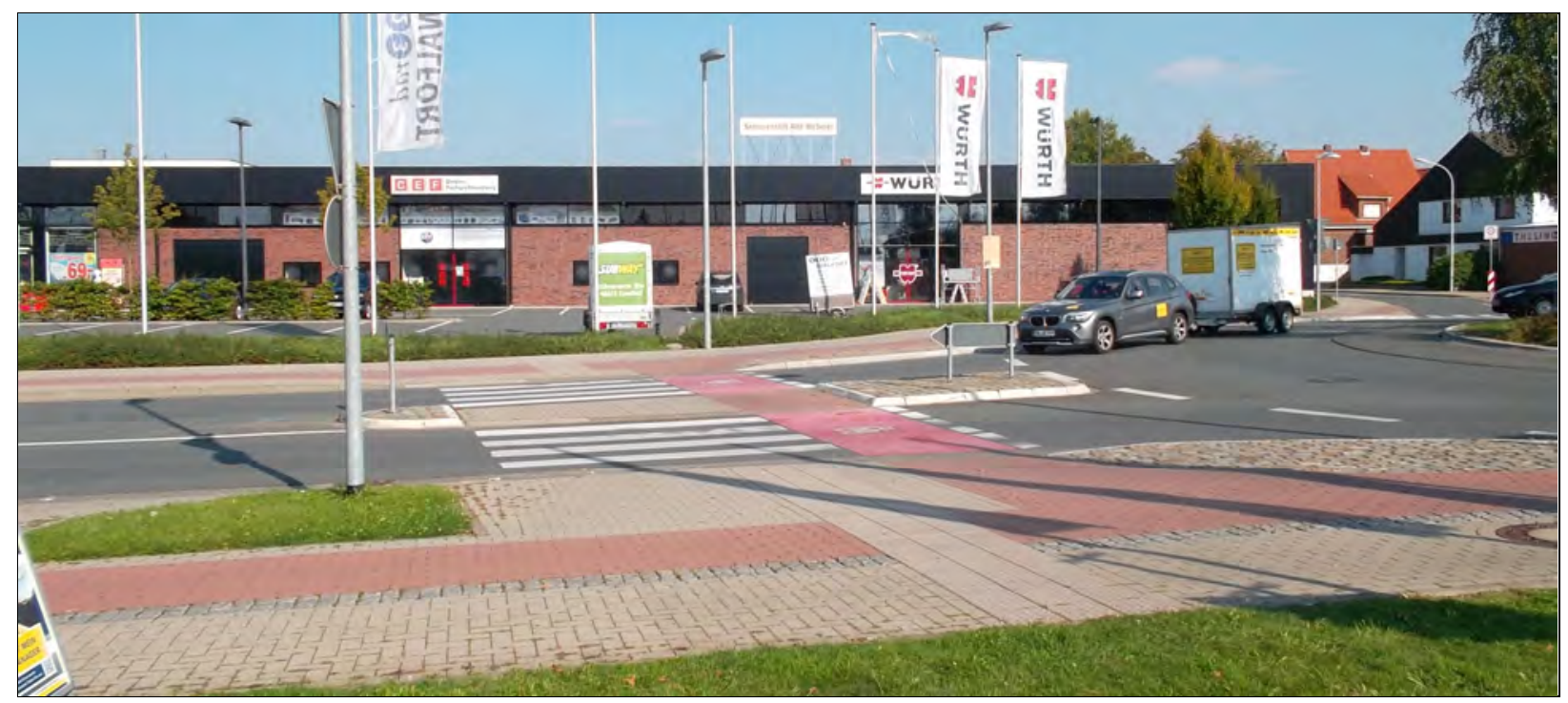

Abbildung 71: Kreisverkehr Coesfeld, Dülmener Straße - Einfärbung der Radfahrerfurten

Die folgende Abbildung zeigt die Unfallentwicklung in den Jahren 2007 bis 2009 vor Umsetzung der Maßnahmen sowie 2011 bis 2013 nach Umsetzung der Maßnahmen. Betrachtet wurden die Unfälle mit Radfahrerbeteiligung der Typen 243, 244, 341 und 342 in allen vier Knotenpunktarmen.

Es zeigt sich, dass die Anzahl der relevanten Radfahrerunfälle der Typen 243, 244, 341 und 342 im Vorher-und Nachher-Zeitraum mit jeweils 2 konstant geblieben ist. Im Nachher-Zeitraum ereigneten sich beide Unfälle mit Jahr 2011.

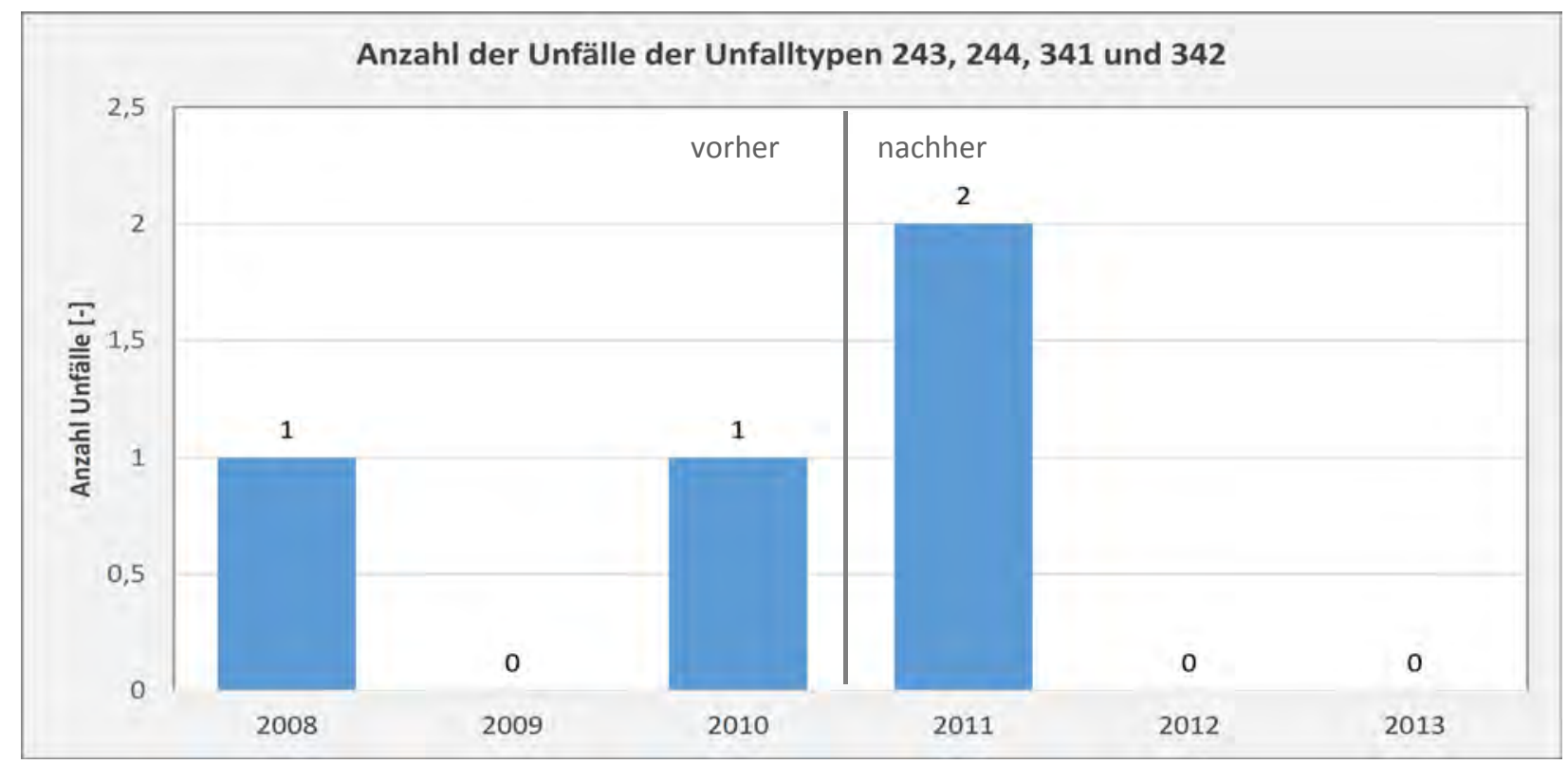

Abbildung 72: Kreisverkehr Coesfeld, Dülmener Straße - Unfallentwicklung 
Die folgenden Abbildungen zeigen die Unfallkenngrößen im Vorher-Nachher-Vergleich getrennt nach Knotenpunktarmen. Es zeigt sich, dass sich die Unfälle im Vorher- und Nachher-Zeitraum ausschließlich auf den nördlichen Knotenpunktarm der Dülmener Straße konzentrieren.

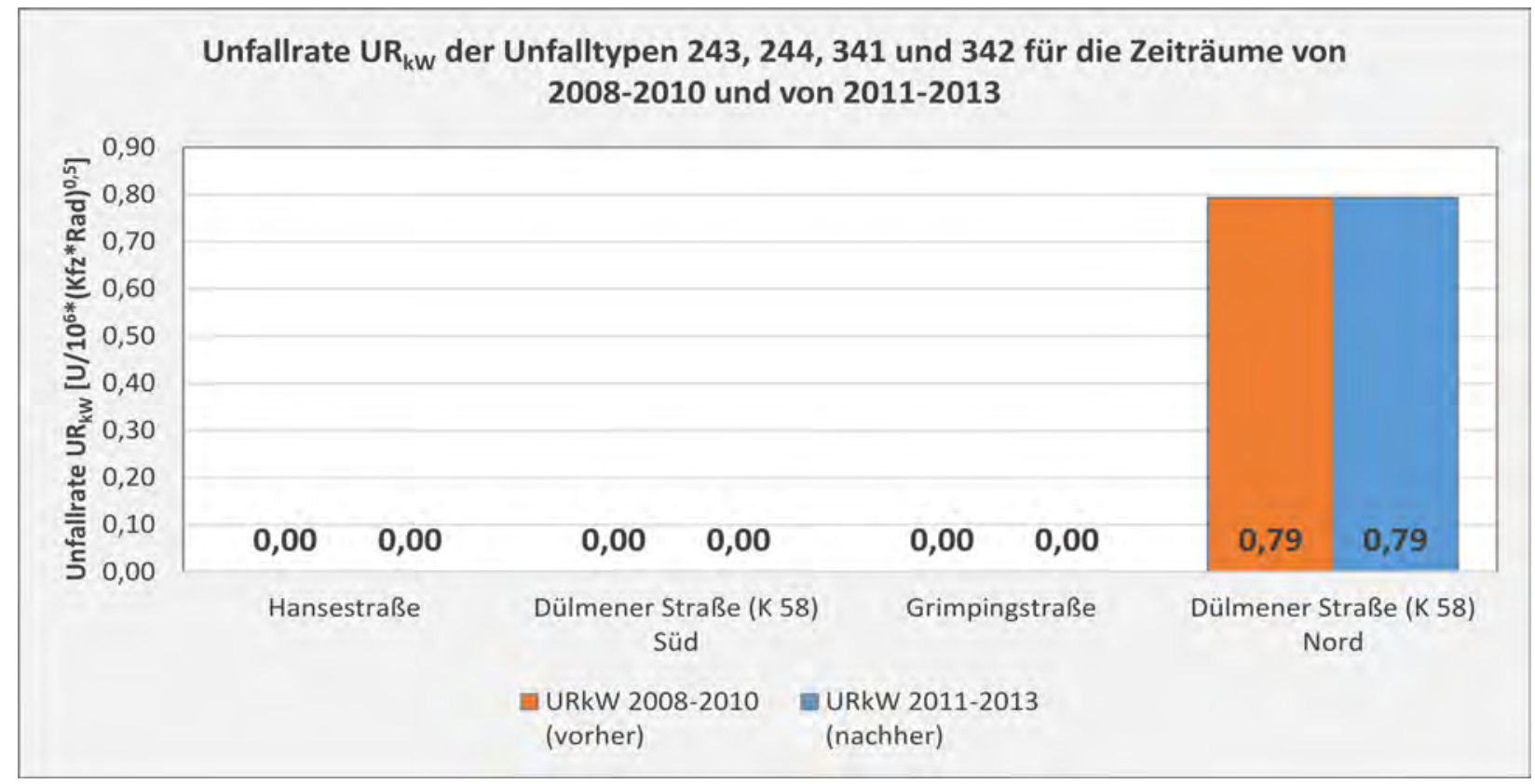

Abbildung 73: Kreisverkehr Coesfeld, Dülmener Straße - Vorher-Nachher-Vergleich, getrennt nach Knotenpunktarmen, $\mathrm{UR}_{\mathrm{kW}}$

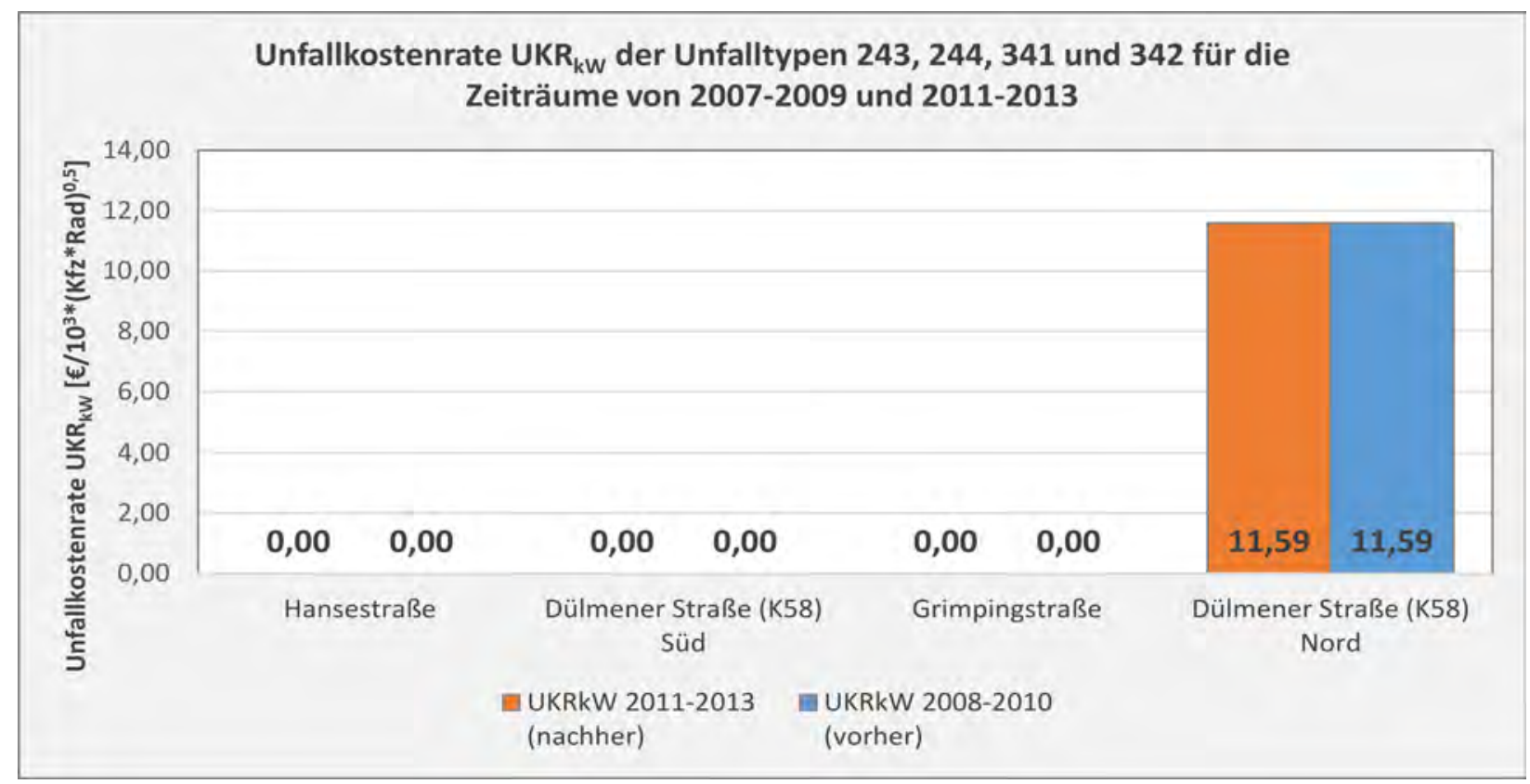

Abbildung 74: Kreisverkehr Coesfeld, Dülmener Straße - Vorher-Nachher-Vergleich, getrennt nach Knotenpunktarmen, $\mathrm{UKR}_{\mathrm{kW}}$

Am Fallbeispiel Coesfeld lässt sich kein Erfolg der Maßnahme nachweisen. Die Unfallzahlen der für die Untersuchung relevanten Unfalltypen sind insgesamt nur gering. 


\section{Verkehrsverhaltensbeobachtungen}

\section{$11.1 \quad$ Methodik}

An 8 Kreisverkehrsarmen wurde das Verkehrsgeschehen an einem Werktag im Zeitraum von 11:00 15:00 Uhr mittels Videokameras erfasst. Die Positionen der Videokameras wurden dabei in Abhängigkeit von den örtlichen Voraussetzungen individuell festgelegt. Dabei wurden möglichst unauffällige Kamerastandorte gewählt, um ein weitgehend unbeeinflusstes Verkehrsverhalten zu gewährleisten.

Die Auswertungen konzentrierten sich auf das Verhalten der Radfahrer sowie auf die Interaktion zwischen Radfahrern und dem Kfz-Verkehr an den Furten. In Abhängigkeit von den vier Fahrmanövern der Radfahrer

- Querung der Ausfahrt gegen den Uhrzeigersinn

- Querung der Ausfahrt im Uhrzeigersinn

- Querung der Zufahrt gegen den Uhrzeigersinn

- Querung der Zufahrt im Uhrzeigersinn

wurden die folgenden Situationen vermerkt:

- Kein Halt / Verzögerung des Radfahrers vor der Querung mit Kfz im Konfliktbereich

- Kein Halt / Verzögerung des Radfahrers vor der Querung ohne Kfz im Konfliktbereich

- Halt / Verzögerung des Radfahrers ohne Kfz im Konfliktbereich

- Absteigen und Schieben des Fahrrads über die Furt

- Halt / Verzögerung des Radfahrers mit Kfz im Konfliktbereich und Vorfahrtverzicht des Radfahrers

- Halt / Verzögerung des Radfahrers mit Kfz im Konfliktbereich und erzwungene Vorfahrt durch Kfz

- Halt / Verzögerung des Radfahrers mit Kfz im Konfliktbereich ohne Vorfahrtverzicht

- Abrupter Bremsvorgang des Kfz

- Beinahe-Unfall 


\subsection{Auswahl der Kreisverkehrsarme}

Für die Vor-Ort-Untersuchungen wurden 8 geeignete Kreisverkehrsarme ausgesucht. Dabei wurden die folgenden Kriterien berücksichtigt:

- eher unauffällige“ Querungsstellen und „besonders auffällige“ Querungsstellen

- Querungsstellen mit zugelassenem Zweirichtungsverkehr und Querungsstellen mit Einrichtungsverkehr

- Querungsstellen mit Aufpflasterung

- Querungsstellen mit Wartelinie in der Ausfahrt

- Winklige Führung und kreisbetonte Führung

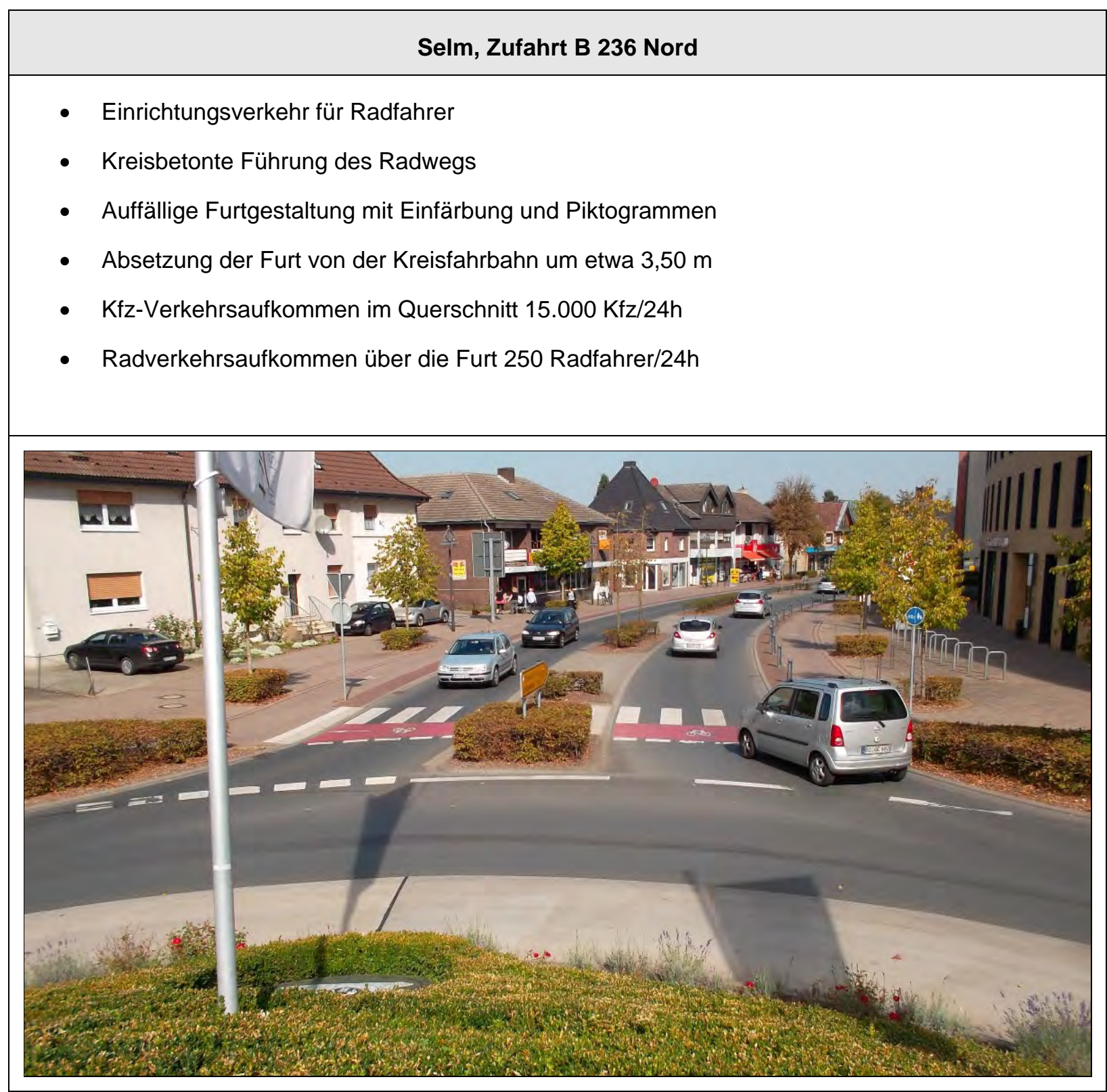

Abbildung 75: Kreisverkehr Selm, Zufahrt B 236 Nord 


\section{Viersen, Zufahrt Krefelder Straße Ost}

- Einrichtungsverkehr für Radfahrer

- Kreisbetonte Führung des Radwegs

- Unauffällige Furtgestaltung ohne Einfärbung und ohne Piktogramme

- Absetzung der Furt von der Kreisfahrbahn um etwa 2,50 m

- $\quad$ Kfz-Verkehrsaufkommen im Querschnitt 8.500 Kfz/24h

- Radverkehrsaufkommen über die Furt 450 Radfahrer/24h

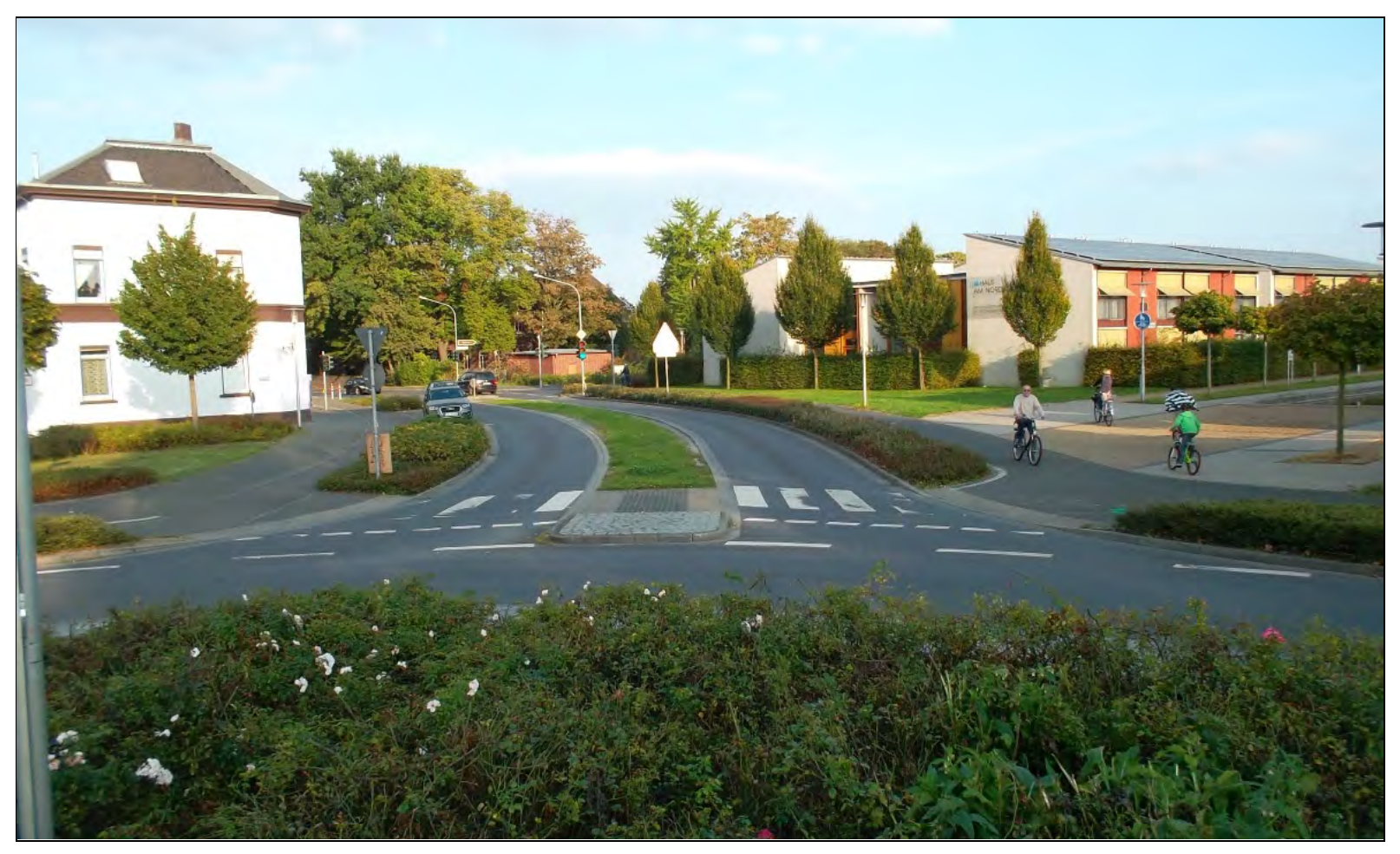

Abbildung 76: Kreisverkehr Viersen, Zufahrt Krefelder Straße Ost 


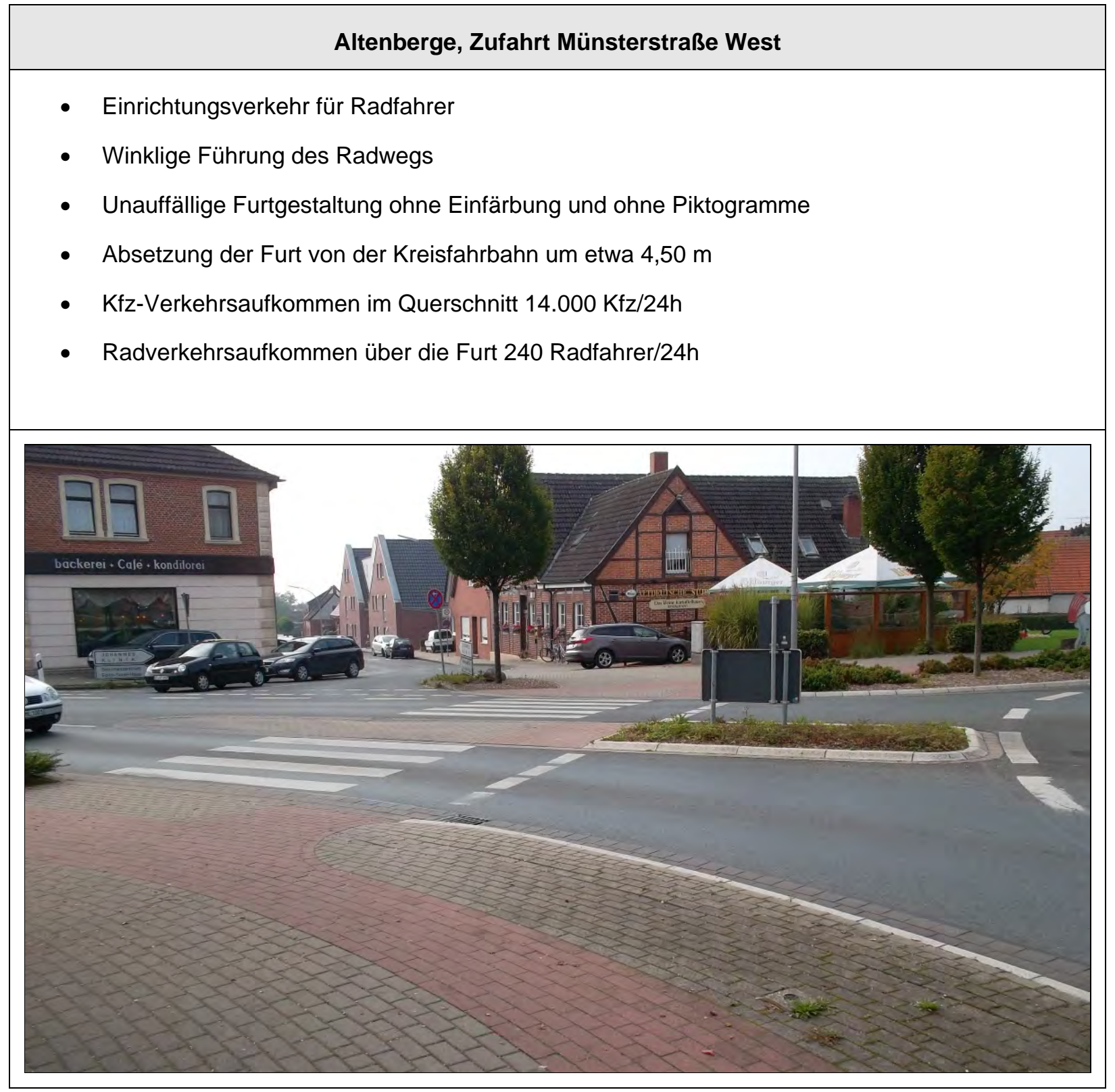

Abbildung 77: Kreisverkehr Altenberge, Zufahrt Münsterstraße West 


\section{Bergheim, Zufahrt Gutenbergstraße Ost}

- Zweirichtungsverkehr für Radfahrer

- kreisbetonte Führung des Radwegs

- Auffällige Furtgestaltung mit Einfärbung und mit Piktogrammen

- Absetzung der Furt von der Kreisfahrbahn um etwa 2,00 m

- Kfz-Verkehrsaufkommen im Querschnitt $11.000 \mathrm{Kfz} / 24 \mathrm{~h}$

- Radverkehrsaufkommen über die Furt 500 Radfahrer/24h

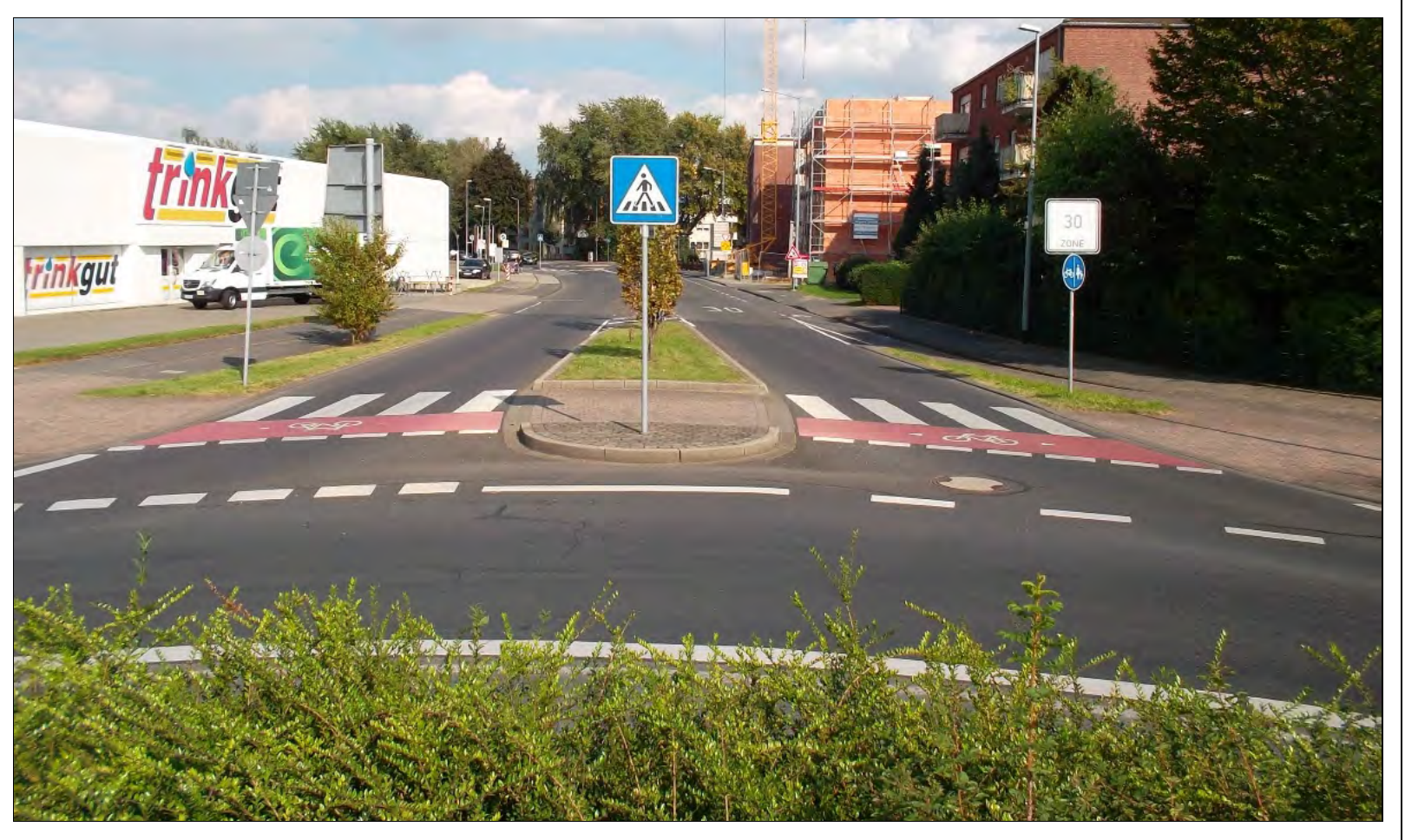

Abbildung 78: Kreisverkehr Bergheim, Zufahrt Gutenbergstraße Ost 


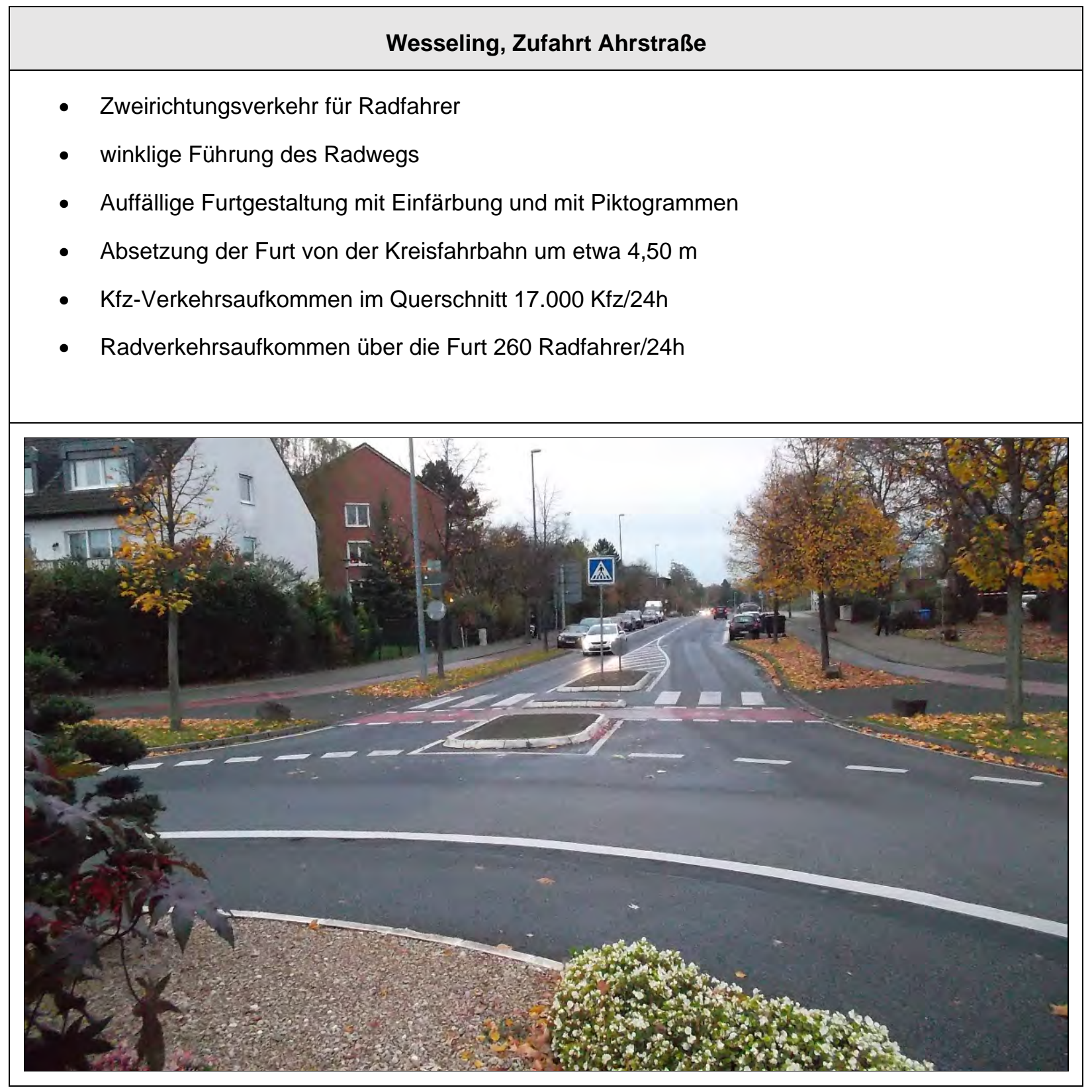

Abbildung 79: Kreisverkehr Wesseling, Zufahrt Ahrstraße 


\section{Soest, Zufahrt Ardeyweg}

- Zweirichtungsverkehr für Radfahrer

- $\quad$ winklige Führung des Radwegs

- Unauffällige Furtgestaltung ohne Einfärbung

- Wartelinie in der Ausfahrt

- Absetzung der Furt von der Kreisfahrbahn um etwa 3,60 m

- Kfz-Verkehrsaufkommen im Querschnitt $8.000 \mathrm{Kfz} / 24 \mathrm{~h}$

- Radverkehrsaufkommen über die Furt 440 Radfahrer/24h

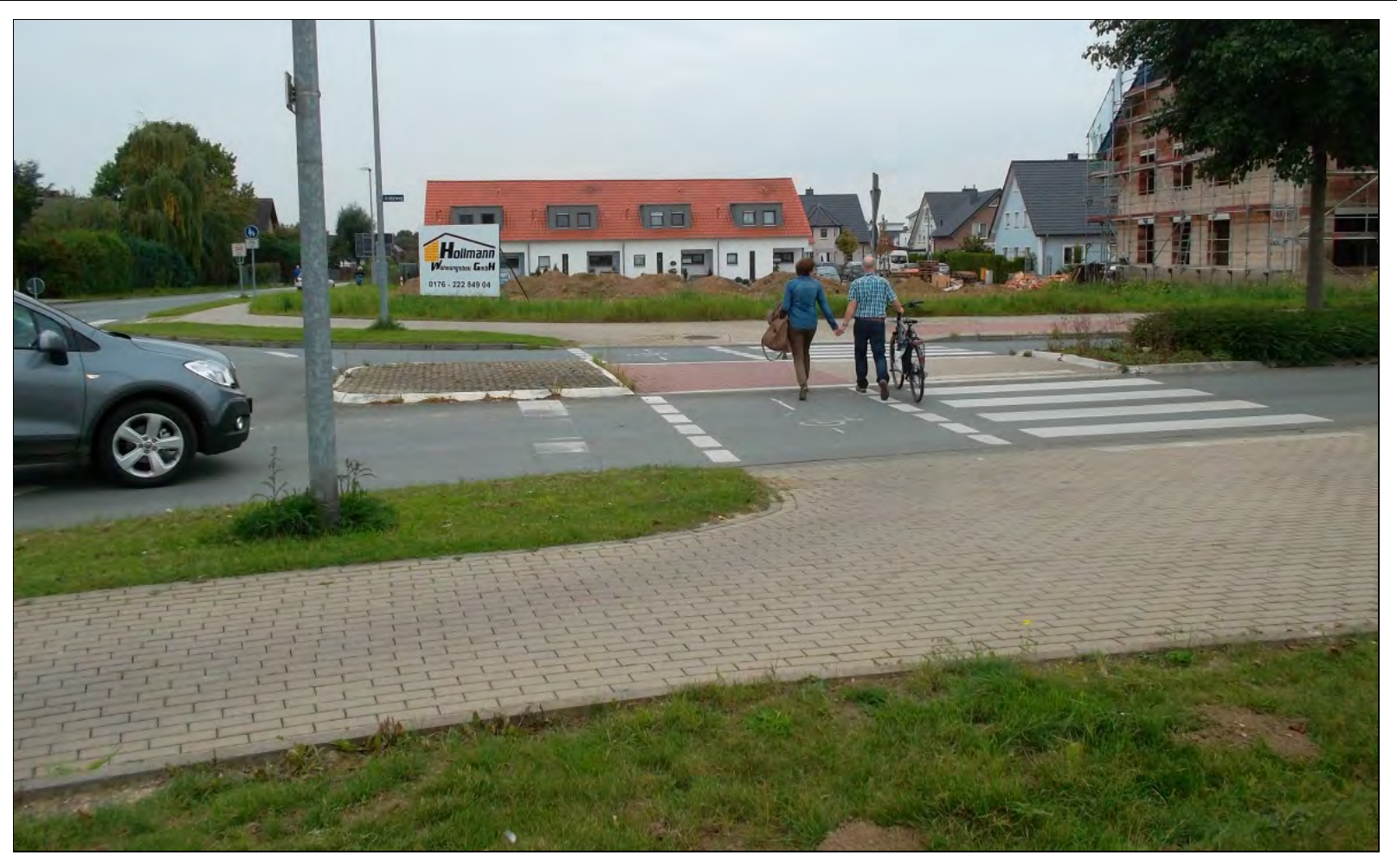

Abbildung 80: Kreisverkehr Soest, Zufahrt Ardeyweg 


\section{Nettetal, Breyeler Straße West}

- Einrichtungsverkehr für Radfahrer

- $\quad$ winklige Führung des Radwegs

- Aufpflasterung

- Absetzung der Furt von der Kreisfahrbahn um etwa 5,00 m

- Kfz-Verkehrsaufkommen im Querschnitt 16.500 Kfz/24h

- Radverkehrsaufkommen über die Furt 400 Radfahrer/24h

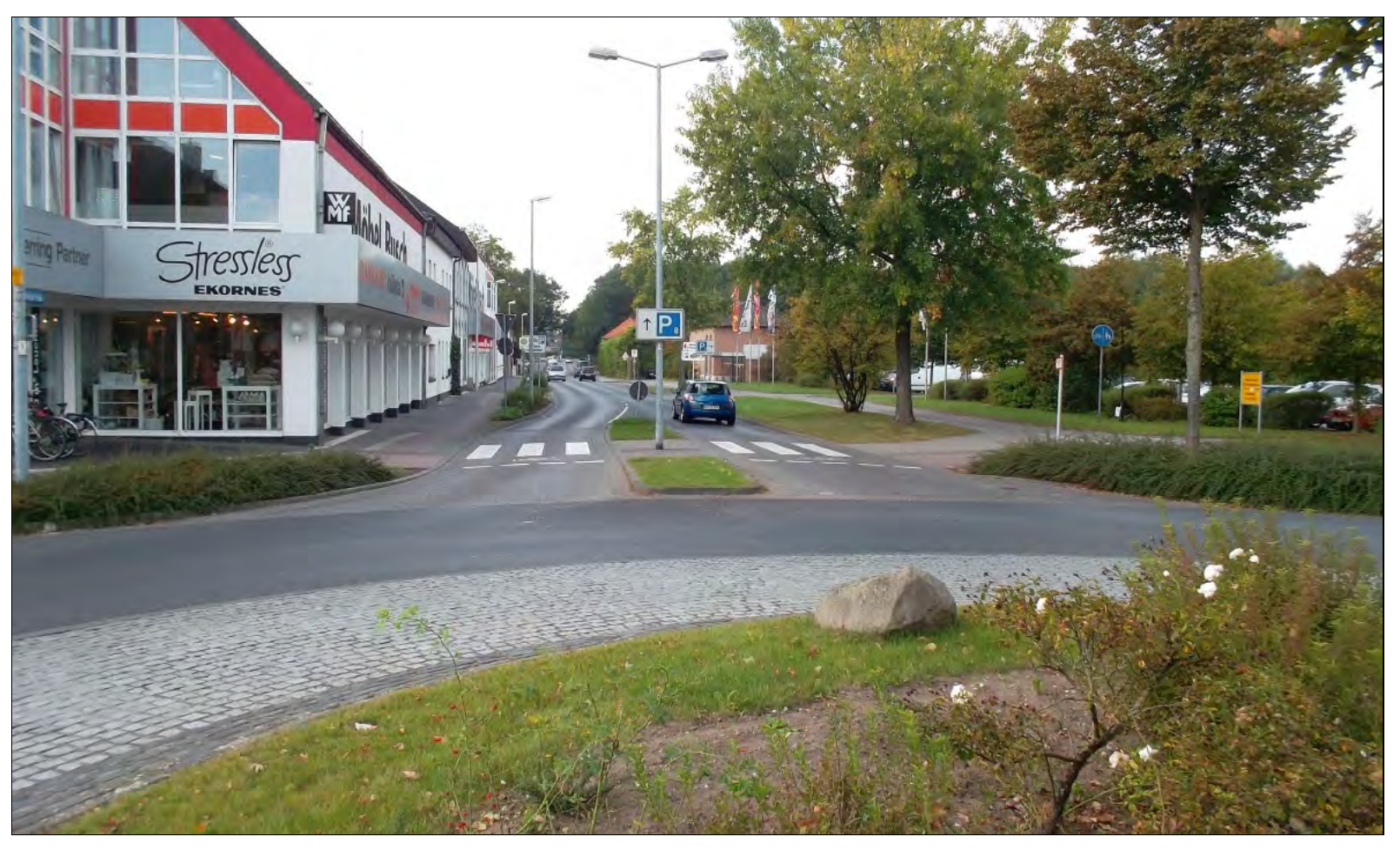

Abbildung 81: Kreisverkehr Nettetal, Breyeler Straße West 


\section{Schwerte, Lohbachstraße}

- Einrichtungsverkehr für Radfahrer

- $\quad$ winklige Führung des Radwegs

- Unauffällige Furtgestaltung ohne Einfärbung

- Wartelinie in der Ausfahrt

- Absetzung der Furt von der Kreisfahrbahn um etwa 3,40 m

- Kfz-Verkehrsaufkommen im Querschnitt 11.500 Kfz/24h

- Radverkehrsaufkommen über die Furt 400 Radfahrer/24h

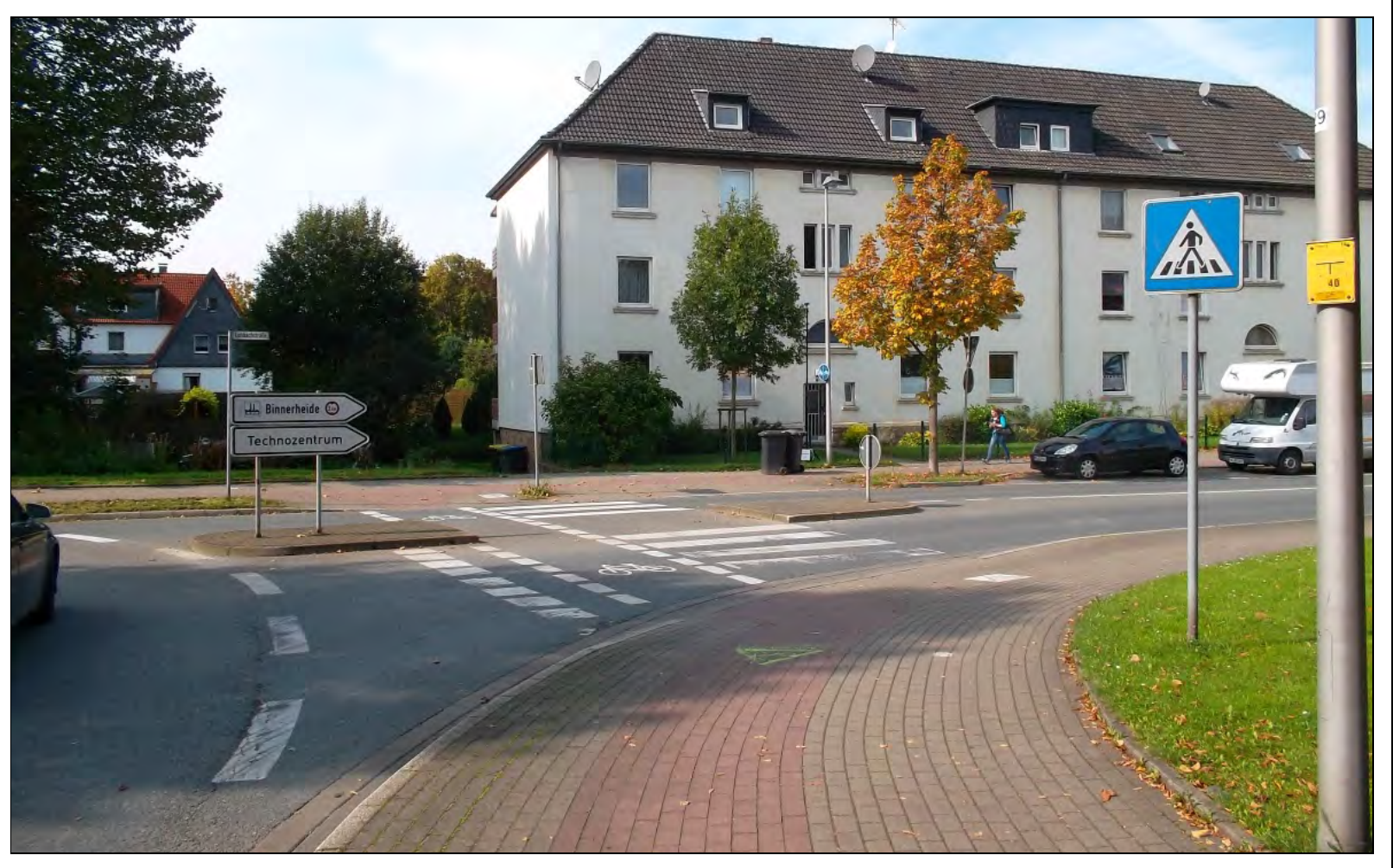

Abbildung 82: Kreisverkehr Schwerte, Lohbachstraße 


\subsection{Ergebnisse}

Die Auswertungen zeigen, dass kein offensichtlicher Zusammenhang zwischen der Gestaltung der Furt und dem Verhalten der Radfahrer besteht.

\section{Gestaltung der Furt}

Es wurde nach den folgenden Kategorien differenziert:

- Auffällige Furt mit Einfärbung:

- Unauffällige Furt ohne Einfärbung:

- Wartelinie in der Ausfahrt:

- Aufpflasterung:
3 Kreisverkehrsarme

2 Kreisverkehrsarme

2 Kreisverkehrsarme

1 Kreisverkehrsarm

Die folgende Abbildung zeigt den Anteil der an der Querungsstelle haltenden oder die Fahrt verzögernden Radfahrer bei einem Kraftfahrzeug im Konfliktbereich. Der Anteil der Radfahrer liegt jeweils auf einem ähnlich hohen Niveau. Tendenziell scheint der Anteil der haltenden oder verzögernden Radfahrer an der Furt mit Aufpflasterung (ein Knotenpunktarm) etwas geringer zu sein.

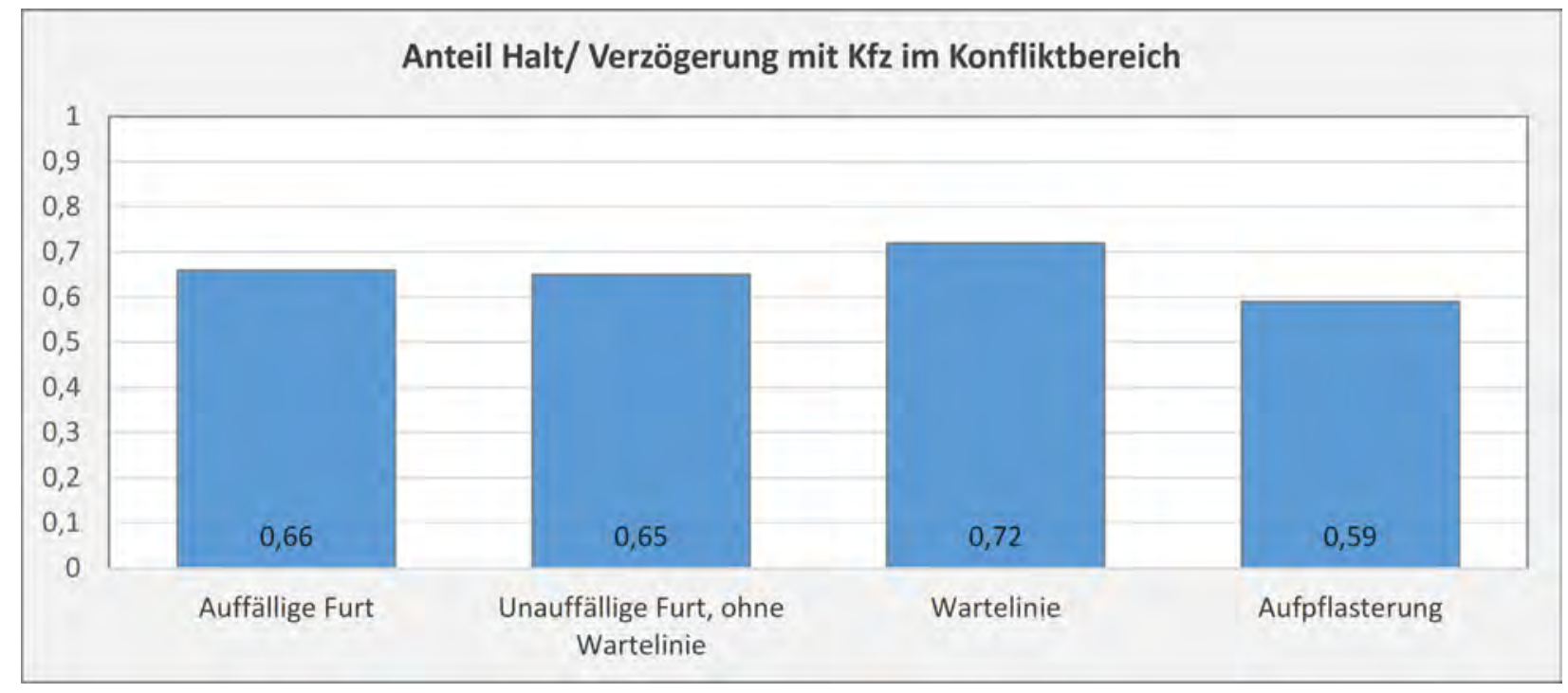

Abbildung 83: Anteil der haltenden / verzögernden Radfahrer mit Kfz im Konfliktbereich

Es ist zu betonen, dass die Werte für die einzelnen Kreisverkehre innerhalb einer Kategorie sehr stark streuen. Bei den auffällig gestalteten Furten weist der Kreisverkehrsarm in Selm mit 0,81 eine ausgesprochen hohe Halt-, Verzögerungsrate auf, wo hingegen der Kreisverkehrsarm in Bergheim mit 0,48 eine unterdurchschnittliche Rate aufweist.

Die folgende Abbildung zeigt den Anteil der an der Querungsstelle haltenden oder die Fahrt verzögernden Radfahrer, wenn sich kein Kraftfahrzeug im Konfliktbereich befindet. Auch hier liegt der Anteil der Radfahrer jeweils auf einem ähnlich hohen Niveau. 


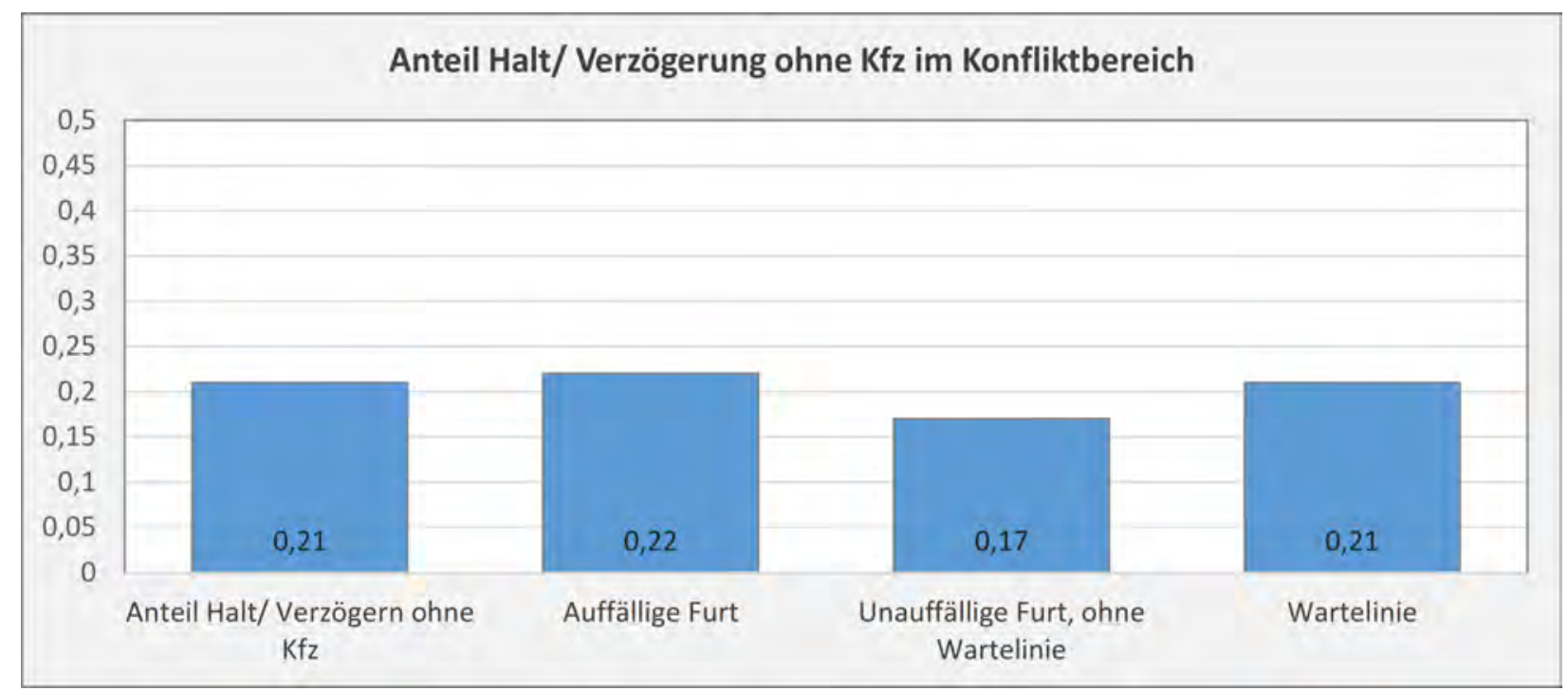

Abbildung 84: Anteil der haltenden / verzögernden Radfahrer ohne Kfz im Konfliktbereich

\section{Führung des Radweges}

Es wurde nach den folgenden Kategorien differenziert:

- Kreisbetonte Führung: 3 Kreisverkehrsarme

- Winklige Führung: 5 Kreisverkehrsarme

Auch die Führung des Radwegs um den Kreisverkehr hat keinen Einfluss auf das Verhalten der Radfahrer. Sowohl bei kreisbetonter als auch bei winkliger Führung liegt der Anteil der haltenden oder verzögernden Radfahrer in der gleichen Größenordnung.

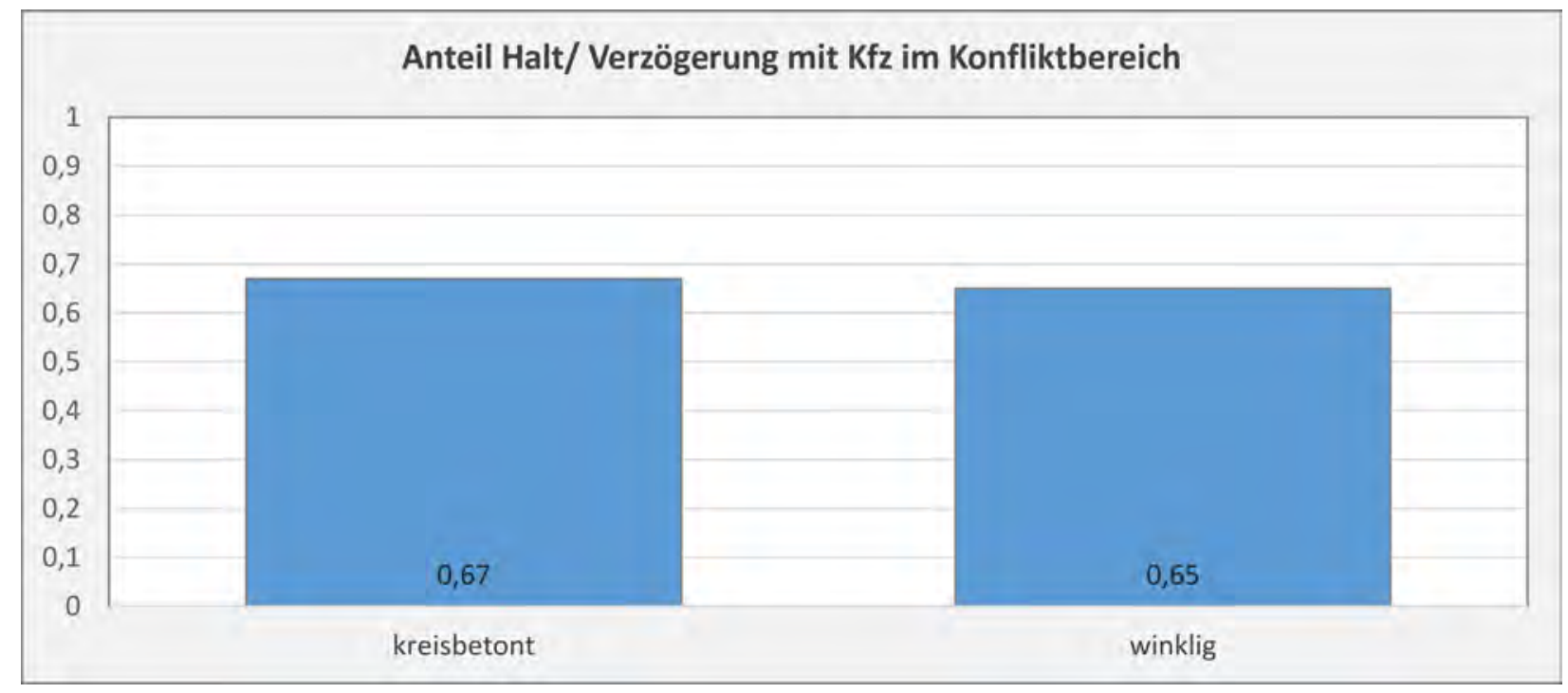

Abbildung 85: Anteil der haltenden / verzögernden Radfahrer mit Kfz im Konfliktbereich 
Auch hier streuen die Werte zwischen den einzelnen Kreisverkehrsarmen einer Kategorie stark. Bei den Kreisverkehrsarmen mit kreisbetonter Radwegeführung reichen die Halte- Verzögerungsraten von 0,48 (Bergheim) bis 0,81 (Selm). Bei den Kreisverkehrsarmen mit winkliger Radwegeführung reichen die Halte- Verzögerungsraten von 0,50 (Altenberge) bis 0,77 (Soest).

Die folgende Abbildung zeigt den Anteil der an der Querungsstelle haltenden oder die Fahrt verzögernden Radfahrer, wenn sich kein Kraftfahrzeug im Konfliktbereich befindet. Auch hier liegt der Anteil der Radfahrer jeweils auf einem ähnlich hohen Niveau.

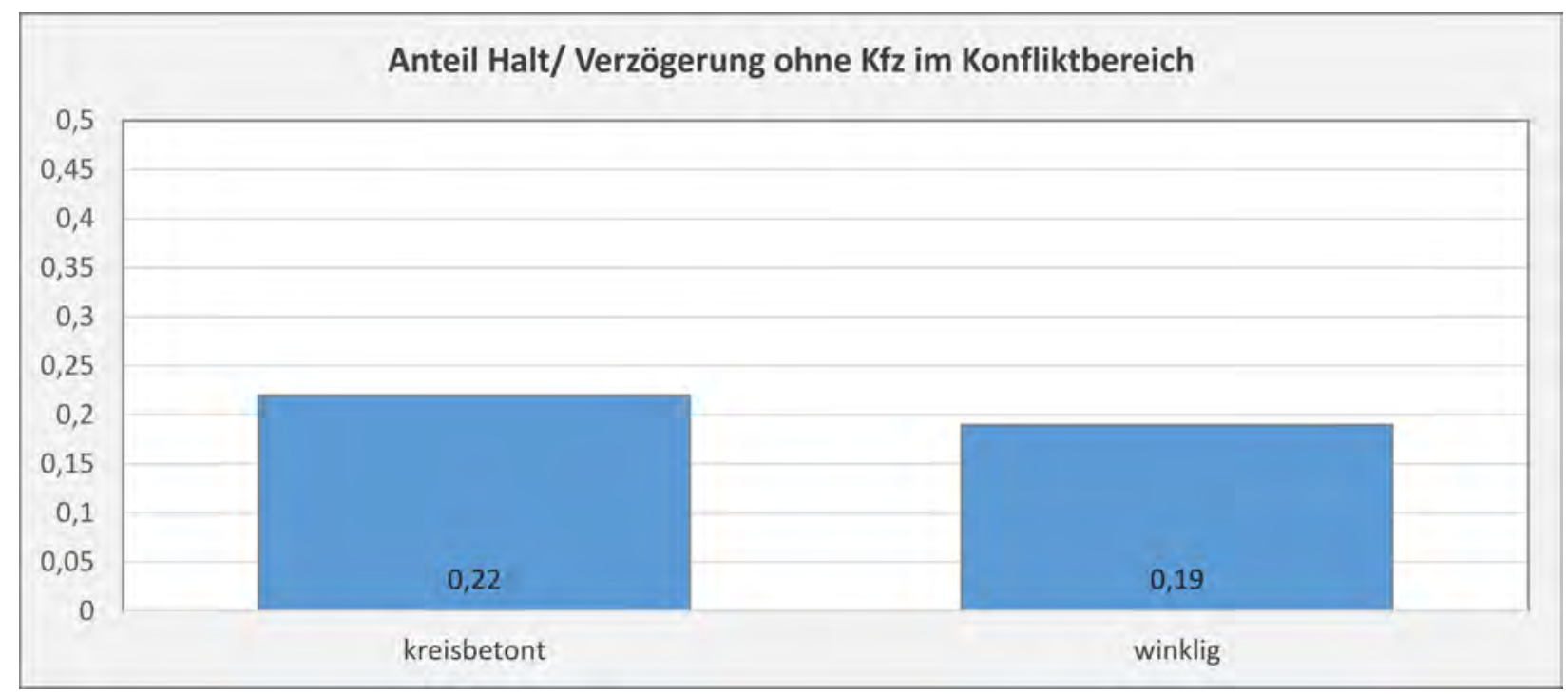

Abbildung 86: Anteil der haltenden / verzögernden Radfahrer ohne Kfz im Konfliktbereich

\section{Vorfahrtverzicht der Radfahrer}

Lediglich am Kreisverkehrsarm in Viersen wurde mit etwa $6 \%$ ein nennenswerter Anteil der Radfahrer beobachtet, die auf ihren Vorrang verzichten. In Altenberge, Schwerte, Soest und Wesseling wurde während des Beobachtungszeitraums kein Vorfahrtverzicht beobachtet.

\section{Verhalten der Kraftfahrer}

Auch bezogen auf das Verhalten der Kraftfahrer sind keine systematischen Unterschiede erkennbar. Der Anteil der abrupten Bremsvorgänge liegt an den einzelnen Querungsstellen zwischen $0 \%$ und $7 \%$. Die höchsten Anteile wurden dabei mit $7 \%$ an den Furten in Altenberge (unauffällige, winklige Führung) und Selm (auffällige, kreisbetonte Führung) beobachtet.

In der gleichen Größenordnung liegt der Anteil der Konflikte mit erzwungenem Vorrang durch die Kraftfahrer. Der höchste Anteil wurde wiederum in Altenberge mit etwa $7 \%$, vor Nettetal mit etwa $5 \%$ beobachtet. 


\section{Zusammenfassung und planerische Empfehlungen}

\subsection{Zusammenfassung}

Die Brilon Bondzio Weiser Ingenieurgesellschaft für Verkehrswesen $\mathrm{mbH}$ hat im Auftrag der Unfallforschung der Versicherer im Jahre 2012 eine Untersuchung zur Sicherheit innerörtlicher Kreisverkehre [1] vorgelegt. Im Rahmen dieser Untersuchung wurde festgestellt, dass umlaufende Radwege mit Bevorrechtigung der Radfahrer an den Querungsstellen deutlich unsicherer sind als andere regelkonforme Radverkehrsführungen. Die Unfallkenngrößen liegen $40 \%$ bis $60 \%$ über denen anderer Radverkehrsführungen.

Umlaufende Radwege stellen jedoch eine weit verbreitete Standardführungsform an Kreisverkehren dar und werden aus einem subjektiven Sicherheitsgefühl heraus häufig gefordert. Vor diesem Hintergrund hat der GDV die Brilon Bondzio Weiser Ingenieurgesellschaft für Verkehrswesen mbH mit einem weiter führenden Forschungsprojekt zur sicheren Führung von Radfahrern auf bevorrechtigten Radwegen an Kreisverkehren beauftragt. Im Rahmen dieses Forschungsprojektes war zu untersuchen, inwieweit durch die Wahl bestimmter Gestaltungs- und Ausstattungselemente ein möglichst hohes Maß an Verkehrssicherheit für Radfahrer an bevorrechtigten Radwegen erreicht werden kann.

Im Rahmen der Untersuchung wurden die folgenden Arbeitsschritte durchgeführt:

- Analyse in- und ausländischer Literatur zur Verkehrssicherheit von Radfahrern an Kreisverkehren

- Analyse des Unfallgeschehens mit Radfahrerbeteiligung an 294 Kreisverkehrsarmen mit vorfahrtrechtlicher Überordnung des Radverkehrs an den Querungsstellen auf der Grundlage der polizeilichen Unfallanzeigen

- Multivariate Analyse des Unfallgeschehens

- Vorher-Nachher-Vergleich an 8 Kreisverkehrsarmen

- Verkehrsverhaltensbeobachtungen an 8 Kreisverkehrsarmen mittels Videoanalyse

\section{Ergebnisse der Unfallanalyse}

Die Unfallanalyse umfasste die Auswertung der polizeilichen Unfallanzeigen an 294 Kreisverkehrsarmen über einen Zeitraum von drei Jahren (2011 bis 2013). Dabei wurden insgesamt 224 Unfälle mit Radfahrerbeteiligung berücksichtigt. Im Rahmen der multivarianten Analyse kamen die Korrelationsrechnungen, das Multivariate lineare Modell und das Multivariate Clusterverfahren zum Einsatz. Die multivariate Unfallanalyse bestätigt die Ergebnisse der Unfallanalyse. Darüber hinaus gehende Erkenntnisse wurden nicht ermittelt.

Hinsichtlich der geometrischen Elemente der Kreisverkehre lassen sich die folgenden Zusammenhänge feststellen:

- Kreisverkehre mit kleinen Außendurchmessern (unter 29,5 m) weisen insbesondere in den Ausfahrten (Unfalltypen 243 (Kraftfahrer biegt aus, Radfahrer kommt von rechts) und 244 (Kraftfahrer biegt aus dem Kreisverkehr aus, Radfahrer kommt von links)) eine höhere 
Gefährdung für Radfahrer in beiden Fahrtrichtungen auf, als Kreisverkehre mit größeren Außendurchmessern. Die Unfallkostenrate für Kreisverkehre mit einem Außendurchmesser unter 29,5 m liegt um etwa 40 \% höher als bei größeren Außendurchmessern.

- Kleine Kreisinseln (unter 12,7 m Durchmesser) führen zu einer um bis zu 65 \% höheren Unfallkostenrate. Insbesondere bei Unfällen des Typs 244 (Kraftfahrer biegt aus dem Kreisverkehr aus, Radfahrer kommt von links) liegt die Unfallkostenrate doppelt so hoch als an Kreisverkehren mit größeren Kreisinseln.

- Die Breite des Innenrings beeinflusst in erster Linie die Unfallschwere in den Ausfahrten. Bei einer Innenringbreite von 3 m und mehr steigt die Unfallkostenkostenrate um bis zu $140 \%$ an.

- Kreisverkehrsarme an Kreisverkehren mit ausschließlich markiertem Innenring sowie mit baulich gestaltetem, aber nur geringfügig überhöhtem Innenringen (bis 2,4 cm) weisen doppelt so hohe Unfallkenngrößen auf, als Kreisverkehrsarme an Kreisverkehren mit einer höheren Absetzung des Innenrings sowie an Kreisverkehren mit einer Innenringgestaltung, die den Fahrtkomfort beeinträchtigt (raue Oberfläche, größere Querneigung).

- Das Vorhandensein von Kunstobjekten auf der Kreisinsel beeinflusst das Unfallgeschehen in der Zufahrt. Bezogen auf den Unfalltyp 341 (Kraftfahrer biegt ein, Radfahrer kommt von links) liegt die Unfallkostenrate bei Kreisverkehren mit Kunst auf der Kreisinsel etwa $60 \%$ über der Unfallkostenrate bei Kreisverkehren ohne Kunstobjekte.

- Es besteht ein leichter Zusammenhang zwischen der Art der Radverkehrsführung und dem Unfallgeschehen. Bezogen auf die Unfallkostenrate liegen die Werte bei gemeinsamen Geh/Radwegen sowie bei Gehwegen mit dem Zusatz Radfahrer frei an den Querungsstellen um bis zu 26\% niedriger als bei separaten Radwegen.

- Kreisverkehrsarme, an denen die Radfahrer im Zulauf auf der Fahrbahn geführt werden und erst vor der Kreisfahrbahn auf einem Radweg geführt werden, weisen an den Querungsstellen halb so hohe Unfallkenngrößen auf als Kreisverkehrsarme mit Radwegen im Zulauf.

- Kreisverkehre mit Radwegen im Einrichtungsverkehr weisen bezogen auf alle Radfahrerunfälle an den Querungsstellen um $20 \%$ höhere Unfallkenngrößen auf, als Kreisverkehre mit Zweirichtungsverkehr. Signifikante Unterschiede zeigen sich beim Unfalltyp 244 (Kraftfahrer biegt aus dem Kreisverkehr aus, Radfahrer kommt von links). Die Unfallkostenrate liegt für diesen Unfalltyp an Kreisverkehren mit Zweirichtungsverkehr viermal höher als bei den Kreisverkehren mit Einrichtungsverkehr. Bei den übrigen Unfalltypen sind keine signifikanten Unterschiede erkennbar.

- Kreisverkehrsarme mit eingefärbten Radfahrerfurten weisen eine um $62 \%$ höhere Unfallrate für Radfahrer auf, als Kreisverkehre ohne Furteinfärbung. Bei der Bewertung dieses Zusammenhangs ist allerdings zu berücksichtigen, dass unfallauffällige Kreisverkehre besonders häufig mit eingefärbten Furten ausgestattet werden, obwohl diese Maßnahme nicht zwangsläufig zu einer Verbesserung führt.

- Bezogen auf alle Kreisverkehrsarme hat das Aufbringen von Radfahrerpiktogrammen keinen positiven Einfluss auf die Verkehrssicherheit von Radfahrern. Tendenziell weisen Radfahrerfurten ohne Piktogramme eine um 20 \% geringere Unfallrate für Radfahrer auf. Dieser Zusammenhang 
ist jedoch statistisch nicht signifikant. Betrachtet man nur die Furten mit Zweirichtungsverkehr, so zeigt sich, dass bei Aufbringen von Piktogrammen mit Fahrtrichtungsangabe die Unfallkostenrate um das Dreifache niedriger liegt, als bei Furten ohne Piktogramme.

- Furtbreiten von unter 1,5 m führen bei Radwegen im Zweirichtungsverkehr zu einer um ein vielfaches höheren Unfallkostenrate. Bei Einrichtungsradwegen haben schmalere Furten hingegen eine halb so hohe Unfallkostenrate. Die Unfallkenngrößen von Furten mit einer Breite von mehr als 2,4 $\mathrm{m}$ liegen unabhängig vom Ein- und Zweirichtungsverkehr im Vergleich zu Furten mit einer Breite von 1,5 m bis 2,4 $\mathrm{m}$ um etwa $50 \%$ höher.

- Bezogen auf alle Unfalltypen ist kein signifikanter Zusammenhang zwischen dem Unfallgeschehen und der Furtabsetzung erkennbar. Lediglich gering abgesetzte Furten $(<2,5 \mathrm{~m})$ weisen eine um ein Drittel höhere Unfallrate auf. Bezogen auf den Unfalltyp 243 (Kraftfahrer biegt aus, Radfahrer kommt von rechts) sind hingegen deutlichere Unterschiede erkennbar. Hier zeigen die Furten mit Absetzungen kleiner 2,5 m dreifach höhere Unfallkenngrößen gegenüber Furten die weiter als $4 \mathrm{~m}$ abgesetzt sind.

- Ein Zusammenhang zwischen dem Bewuchs der Beete und dem Unfallrisiko für Radfahrer ist nicht erkennbar.

- Es wurden an jedem Kreisverkehrsarm diejenigen Punkte definiert, an denen die tatsächliche Fahrtrichtung des Radfahrers für den Kraftfahrer erkennbar wird. Beim Unfalltyp 243 (Kraftfahrer biegt aus, Radfahrer kommt von rechts) zeigt sich, dass bei einem besonders weit entfernten Verzweigungspunkt in der Ausfahrt (über $4 \mathrm{~m}$ ) die Unfallkenngrößen um ein vielfaches ansteigen. Die günstigsten Werte werden bei einer sehr kreisnahen Führung erreicht. Beim Verzweigungspunkt in der Zufahrt zeigt sich kein Zusammenhang zwischen dem Unfalltyp 342 (Kraftfahrer biegt ein, Radfahrer kommt von rechts) und der Lage des Verzweigungspunktes.

- Der Abstand des Radwegs vom Kreisrand beeinflusst die Erkennbarkeit des Radfahrers für den aus der Kreisfahrbahn ausfahrenden Kraftfahrer. Ein geringer Abstand begünstigt die Erkennbarkeit. Es zeigt sich, dass bei Absetzungen von $4 \mathrm{~m}$ und mehr die Unfallkostenrate des Typs 243 (Kraftfahrer biegt aus, Radfahrer kommt von rechts) um mindestens das Fünffache ansteigt. Die auf den Unfalltyp 243 bezogen besonders günstige Unfallkenngrößen treten bei eng anliegenden Radwegen verbunden mit einer winkligen Führung des Radweges um den Kreisverkehr auf.

- An acht Kreisverkehrsarmen des Untersuchungskollektivs sind die Querungsstellen baulich besonders gestaltet (Plateauausbildung, bzw. Aufpflasterung). Bei der Unfallanalyse zeigen sich keine signifikanten Unterschiede. Dabei ist zu berücksichtigen, dass die Stichprobengröße nur gering ist.

- An zwölf Kreisverkehrsarmen des Untersuchungskollektivs sind in den Ausfahrten Wartelinien vor den Querungsstellen markiert. Bezogen auf die Unfallschwere besteht kein Zusammenhang zwischen der Wartelinie und den Unfallkenngrößen. Die Unfallkostenraten sind nahezu identisch. Die Unfallraten zeigen bei den Ausfahrten ohne Wartelinien um ein Drittel günstigere Werte. Der Erfolg als Maßnahme zur Reduzierung der Unfälle mit Radfahrern lässt sich anhand des geringen Teilkollektivs nicht nachweisen. 
- An Kreisverkehren werden an den Zufahrten die Zeichen 205 und 215 StVO angeordnet. Bei Radwegefurten werden die Verkehrszeichen im Allgemeinen vor der Querungsstelle angeordnet. Im vorliegenden Untersuchungskollektiv waren an 260 Kreisverkehrszufahrten die Verkehrszeichen vor der Querungsstelle und an 34 Kreisverkehrszufahrten hinter der Querungsstelle angeordnet. Bei der Unfallauswertung wurden nur die Unfalltypen 341 (Kraftfahrer biegt ein, Radfahrer kommt von links) und 342 (Kraftfahrer biegt ein, Radfahrer kommt von rechts) berücksichtigt. Dabei zeigt sich, dass die Kreisverkehrszufahrten mit Anordnung der Verkehrszeichen vor der Querungsstelle eine um $46 \%$ höhere Unfallrate aufweisen. Eine Erklärung dieses Zusammenhangs ist mit den vorliegenden Daten nicht möglich. Hier sind weitergehende Untersuchungen erforderlich.

- Bei Furten im Zweirichtungsverkehr werden in den Zufahrten in der Regel Zusatzzeichen angebracht, die die sich nähernden Kraftfahrer auf den Zweirichtungsverkehr hinweisen. An lediglich 12 von 87 Kreisverkehrsarmen erfolgte kein Hinweis mittels Zusatzzeichen. An diesen 12 Kreisverkehrsarmen traten keine Unfälle des Typs 341 (Kraftfahrer biegt ein, Radfahrer kommt von links) und 342 (Kraftfahrer biegt ein, Radfahrer kommt von rechts) auf. Ein Zusammenhang zwischen Zusatzzeichen in der Zufahrt und Unfallgeschehen ist anhand des Datenkollektivs nicht erkennbar.

- In den Ausfahrten sind lediglich an 6 von 87 Kreisverkehrsarmen Zusatzzeichen mit Hinweis auf den Zweirichtungsverkehr angebracht. Während bezogen auf die Unfallrate keine Unterschiede erkennbar sind, zeigt sich bei den Kreisverkehrsarmen mit Zusatzzeichen eine dreifach höhere Unfallkostenrate. Bei der Bewertung dieses Zusammenhangs ist allerdings zu berücksichtigen, dass eher unfallauffällige Kreisverkehre nachträglich mit Zusatzzeichen ausgestattet werden. Die Beschilderung ist daher aller Voraussicht nach nicht die Ursache für ein höheres Unfallrisiko. Zudem ist die Fallzahl mit nur 6 Kreisverkehrsarmen sehr gering. Als Maßnahme zur Reduzierung der Unfälle mit Radfahrern erscheint diese Maßnahme jedoch eher ungeeignet.

\section{Ergebnisse des Vorher-Nachher-Vergleichs}

Bei den beiden Kreisverkehren Ahaus, Adenauerring und Coesfeld, Dülmener Straße wurden im Jahr 2010 Maßnahmen zur Erhöhung der Verkehrssicherheit für Radfahrer vorgenommen. Für beide Kreisverkehre lagen aus der Untersuchung aus 2012 [1] die Unfalldaten für den Vorher-Zeitraum der Jahre 2007 bis 2009 vor.

Am Kreisverkehr Adenauerring in Ahaus wurden die Querungsstellen für Fußgänger und Radfahrer im Verlauf des Jahres 2010 durch Plateaus in etwa auf das Niveau der Geh- und Radwege angehoben. Zudem wurde eine Neutrassierung des Radweges im Bereich zwischen Adenauerring und Wessumer Straße West vorgenommen. Im Vorfeld der Baumaßnahme fand bereits ein Rückschnitt des kreisnahen Bewuchses statt. Zusammenfassend sind die umgesetzten Maßnahmen als erfolgreich zu bewerten. Die Gesamtzahl der relevanten Unfälle mit Radfahrerbeteiligung ist um $40 \%$ zurückgegangen. Die einzige Ausnahme bildet der westliche Arm der Wessumer Straße mit einer Erhöhung der Unfallzahlen.

Am Kreisverkehr Dülmener Straße in Coesfeld wurden die Radfahrerfurten im Jahr 2010 rot eingefärbt. Die Unfallauswertung zeigt, dass die Anzahl der relevanten Radfahrerunfälle der Typen 243, 244, 341 
und 342 im Vorher-und Nachher-Zeitraum mit jeweils 2 konstant geblieben ist. Am Fallbeispiel Coesfeld lässt sich kein Erfolg der Maßnahme nachweisen. Die Unfallzahlen der für die Untersuchung relevanten Unfalltypen sind insgesamt nur gering.

\section{Ergebnisse der Verhaltensbeobachtungen}

An 8 Kreisverkehrsarmen wurden das Verhalten der Kraftfahrer und der Radfahrer sowie die Interaktionen an den Querungsstellen mittels Videokameras erfasst.

Dabei wurden die folgenden Kriterien berücksichtigt:

- „eher unauffällige“ Querungsstellen und „besonders auffällige“ Querungsstellen

- Querungsstellen mit zugelassenem Zweirichtungsverkehr und Querungsstellen mit Einrichtungsverkehr

- Querungsstellen mit Aufpflasterung

- Querungsstellen mit Wartelinie in der Ausfahrt

- Winklige Führung und kreisbetonte Führung

Die Analyse führt zu den folgenden Ergebnissen:

- Obwohl eine Roteinfärbung unsicherer ist, zeigen die Auswertungen, dass kein offensichtlicher Zusammenhang zwischen der Gestaltung der Furt und dem Verhalten der Radfahrer besteht.

- Auch die Führung des Radwegs um den Kreisverkehr hat keinen Einfluss auf das Verhalten der Radfahrer. Sowohl bei kreisbetonter als auch bei winkliger Führung liegt der Anteil der haltenden oder verzögernden Radfahrer in der gleichen Größenordnung.

- Auch bezogen auf das Verhalten der Kraftfahrer sind keine systematischen Unterschiede erkennbar. Der Anteil der abrupten Bremsvorgänge liegt an den einzelnen Querungsstellen zwischen

$0 \%$ und $7 \%$. In der gleichen Größenordnung liegt der Anteil der Konflikte mit erzwungenem Vorrang durch die Kraftfahrer.

- Insgesamt zeigen die einzelnen Kreisverkehre sehr stark streuende Werte. Das Verhalten ist daher eher durch lokale Besonderheiten geprägt, als durch Gestaltungsmerkmale der Furten. 


\subsection{Planerische Empfehlungen}

Aus den vorliegenden Forschungsergebnissen lassen sich die folgenden Empfehlungen ableiten, die bei der Überarbeitung des Regelwerks für die Anlage von Kreisverkehren Berücksichtigung finden sollten:

\section{Geometrische Gestaltung des Kreisverkehrs}

- Kreisverkehre mit einem Außendurchmesser kleiner als 30 Meter und Innenringe mit einer Breite von mehr als 3 Metern sollten nach Möglichkeit vermieden werden.

- Um eine wirksame Geschwindigkeitsreduzierung der einfahrenden Kraftfahrzeuge zu erreichen, sollten Kreisinseln einen Durchmesser von mindestens 13 Metern erhalten.

- Innenringe sollten geschwindigkeitsdämpfend ausgebildet werden. In Frage kommen höhere Absetzungen von mindestens $3 \mathrm{~cm}$ und / oder eine besonders raue Ausbildung. Positive Erfahrungen liegen auch mit farblich kontrastreicher Gestaltung und mit einer gegenüber der asphaltierten Kreisfahrbahn größeren Querneigung vor.

\section{Sichtbeziehungen zwischen Kraftfahrern und Radfahrern}

- Radwege sollten in den Zufahrten zum Kreisverkehr bereits im Vorfeld fahrbahnnah geführt werden. Insbesondere in den Ausfahrten ist eine fahrbahnnahe Führung wegen der besseren Sichtbeziehungen zwischen Radfahrern und Kraftfahrern stets anzustreben.

- Die Führung des Radwegs um den Kreisverkehr sollte ebenfalls möglichst fahrbahnnah erfolgen. Als vorteilhaft erweist sich eine eng an die Kreisfahrbahn anliegende winklige Führung der Radwege. Die Absetzung zum Fahrbahnrand sollte weniger als 2 Meter betragen und in keinem Fall größer als 4 Meter sein.

\section{Gestaltung der Furt}

- Die Furten sollten um 4 bis $5 \mathrm{~m}$ vom Kreisrand abgesetzt werden.

- Furten im Einrichtungsverkehr sollten eine Breite zwischen 1,50 m und 2,0 m haben.

- Ein Zweirichtungsverkehr für Radfahrer sollte grundsätzlich vermieden werden. Sollte dies nicht möglich sein, so sollten die Furten mindestens 2 Meter breit sein und mit Piktogrammen und Angabe der Fahrtrichtungen versehen werden.

- Die Roteinfärbung von Furten hat keinen positiven Einfluss auf die Verkehrssicherheit der querenden Radfahrer. 


\section{Literaturverzeichnis}

[1] Bondzio, L.; Ortlepp, J; Voss, H.; Scheit, M. [2012]:

Sicherheit innerörtlicher Kreisverkehre. Forschungsprojekt im Auftrag des GDV. Bochum, Berlin.

[2] Alrutz, D. et al. [1992]:

Untersuchung der Verkehrssicherheit für Fußgänger und Radfahrer in Kreisverkehrsplätzen der neuen Generation. Untersuchung im Auftrag des Ministeriums für Stadtentwicklung und Verkehr des Landes Nordrhein-Westfalen. Hannover.

[3] Brilon, W.; Drews, O.; Stuwe, B. [1993]:

Sicherheit und Leistungsfähigkeit von Kreisverkehrsplätzen. FE Nr. 77359/91 im Auftrag des Bundesministers für Verkehr. Bochum..

[4] Brilon, W. [1997]:

Sicherheit von Kreisverkehrsplätzen. In: Zeitschrift für Verkehrssicherheit 43 ( 1997), S. 22-28.

[5] Haller, W.; Lange, J.; Alrutz, D.; Stellmacher-Hein, J. [2000]:

Fußgänger- und Radverkehrsführung an Kreisverkehrsplätzen. Schriftenreihe Straßenbau und Straßenverkehrstechnik, Heft 793. Bonn.

[6] Minnen, J. van [1995]:

Rotondes en voorrangsregelingen. R-95-58. Stichting Wetenschappelijk Onderzoek Verkeersveiligheid SWOV. Leidschendam.

[7] Minnen, J. van [1998]:

Rotondes en voorrangsregelingen II. R-98-12. Stichting Wetenschappelijk Onderzoek Verkeersveiligheid SWOV. Leidschendam.

[8] Weijermars, W. [2001]:

Voorang aan veiligheid op rotondes. Een onderzoek naar de veiligheid van verschillende voorrangsregelingen voor fietseeers op rotondes met vrijliggende fietspaden. Afstudeerscriptie. Universiteit Twente. Enschede.

[9] Daniels, S.; Nuyts, E.; Wets, G. [2008]:

The effects of roundabouts on the traffic safety for bicyclists. An observational study. In: Accident analysis and prevention 40(2), S, . $518-526$. 
[10] Daniels, S.; Brijs, T., Nuyts, E.; Wets, G. [2009]:

Injury crashes with bicyclists at roundabouts: Influence of some location characteristics and the design of cycle facilities. In: Journal of Safety Research 40 (2009), S. $141-148$.

[11] Hels, T.; Orozova-Bekkevold, I. [2007]:

The effect of roundabout design features on cyclist accident rate. In: Accident analysis and prevention 39(2007), S. $300-307$.

[12] Jensen, S.U. [2015]:

Safe roundabouts for cyclists. International Cycling Safety Conference 2015. Hanover.

[13] Brüde, U.; Larsson, J. [2000]:

What roundabout design provides the highest possible Safety? In: Nordic Road and Transport Research 2 (2000), S. $17-32$.

[14] Sakshaug, L.; Laureshyn, A.; Svensson, A.; Hydén, C. [2010]:

Cyclists in roudanbouts - Different design solutions. In. Accident analysis und prevention 42 (2010), S. $1338-1351$.

[15] Forschungsgesellschaft für Straßen- und Verkehrswesen [2006]:

Merkblatt für die Anlage von Kreisverkehren. Bonn.

[16] Vereinigung Schweizerischer Straßenfachleute (VSS) [1999]:

Schweizer Norm SN 640252 „Knoten Führung des leichten Zweiradverkehrs“.

[17] Bundesministerium für Verkehr, Innovation und Technologie Österreich [2010]: Plangleiche Knoten - Kreisverkehre RVS 03.05.14. Wien.

[18] Österreichische Forschungsgesellschaft Straße - Schiene - Verkehr [2014]: Radverkehr RVS 03.02.13. Wien.

[19] CROW [1998]:

Eenheit in rotondes. Publicatie 126. Ede.

[20] CROW [2002]:

Fietsvorsteken op rotondes. Supplement bij publicatie 126 "Eenheit in rotondes". Ede

[21] R Core Team [2014]:

R: A language and environment for statistical computing. R Foundation for Statistical Computing, Vienna, Austria. URL http://www.R-project.org/ 
Gesamtverband der Deutschen Versicherungswirtschaft e. V.

Wilhelmstraße 43 / 43G, 10117 Berlin

Postfach 0802 64, 10002 Berlin

Telefon 030 / 2020 - 50 00, Fax 030 / 2020 - 6000

Internet: www.gdv.de, www.udv.de 